\title{
DGFM M
}

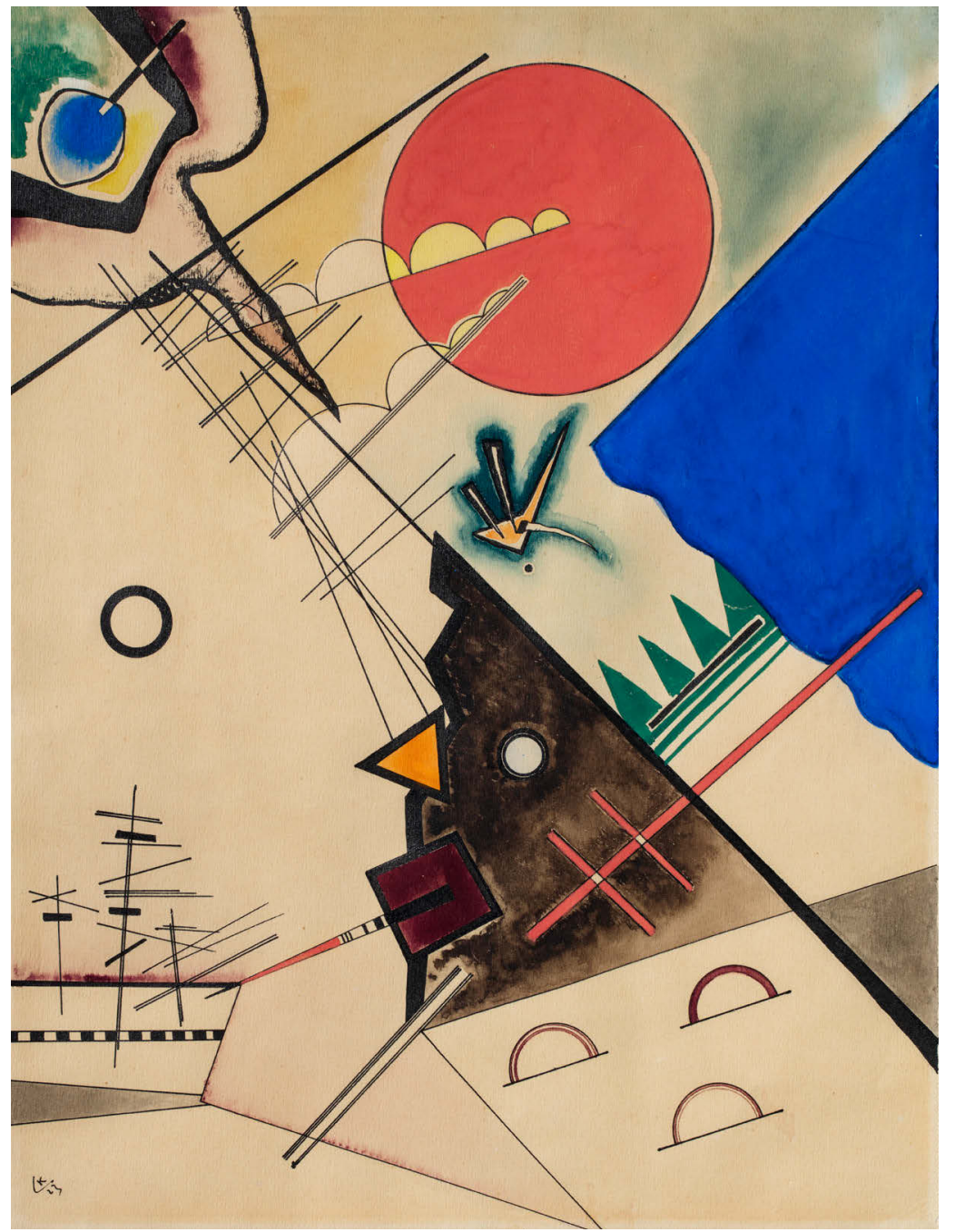

OWassily Kandinsky „Entstehende Verbindung (Roter Kreis)“. Museum für Kunst und Kulturgeschichte Marburg

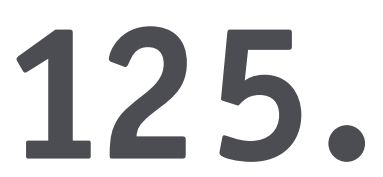

Kongress der

Deutschen Gesellschaft für Innere Medizin e.V.

RheinMain CongressCenter . Wiesbaden

4. - 7. Mai 2019

\section{Abstractband}



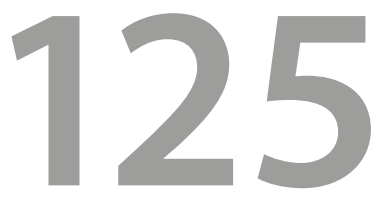

\section{Kongress}

Internist 2019 - 60 (Suppl 1): S1-S69

https://doi.org/10.1007/s00108-019-0612-9

Online publiziert: 18. April 2019

( ) Springer Medizin Verlag GmbH, ein Teil von Springer Nature 2019

\section{Wissenschaftliche Organisation}

\section{Kongress-Präsident}

Prof. Dr. med. Claus F. Vogelmeier

\section{DGIM-Kongress-Team 2019}

Prof. Dr. med. Peter Alter

Dr. med. Björn Beutel

PD Dr. med. Timm Greulich

Dr. med. Angélique Holland

Dr. med. Andreas Jerrentrup

Dr. med. Andreas Klemmer

Prof. Dr. med. Rembert Koczulla

\section{Kongress-Sekretariat}

Karin Hüge-Ruppel

Universitätsklinikum Gießen und

Marburg GmbH

Standort Marburg

Klinik für Innere Medizin

Baldingerstraße - 35043 Marburg

Inge Kokot und

Dr. Sandra Söhler

Philipps-Universität Marburg

Baldingerstraße - 35043 Marburg
DGIM-Geschäftsstelle

DG? Irenenstraße 1

Deutsche Gesellschaft
fur lnnere Medizi 65189 Wiesbaden

Geschäftsführung

RA Maximilian Broglie

Stellvertretende Geschäftsführerin

Dipl.-Kffr. Ourania Menelaou

Managementassistentin

Nicole Safenauer

Kongress-Sekretärin der DGIM

Katharina Wick

Verantwortlich für Patiententag

Dr. med. Norbert Schütz

Telefon: +49 611 - 2058040 - 0

Telefax: +49 611 - 2058040 - 46

info@dgim.de -www.dgim.de

Kongress Agentur (PCO)

m:con - mannheim:congress $\mathrm{GmbH}$

Thilo Hübner

Rosengartenplatz 2

D-68161 Mannheim

www.mcon-mannheim.de

\section{Deutsche Gesellschaft \\ für Innere Medizin \\ DGFM}




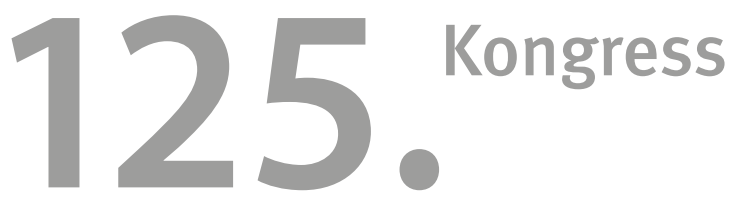

\section{Programm - Kommission DGIM 2018}

Vogelmeier, Claus, Prof. Dr. med., Marburg

(Vorsitzender)

Albus, Christian, Prof. Dr. med., Köln

Alscher, Mark D., Prof. Dr. med., Stuttgart

Alter, Peter, Prof. Dr. med., Marburg

Assmus, Birgit, Prof. Dr. med., Frankfurt

Beutel, Björn, Dr. med., Marburg

Denkinger, Michael, Prof. Dr. med., Ulm

Floege, Jürgen, Prof. Dr. med., Aachen

Greulich, Timm, PD Dr. med., Marburg

Hach-Wunderle, Viola, Prof. Dr. med., Frankfurt

Heppner, Hans Jürgen, Prof. Dr. med., Schwelm

Holland, Angelique, Dr. med., Marburg

Hoyer, Bimba F., Prof. Dr. med., Kiel

Hübner, Thilo, Mannheim

Jerrentrup, Andreas, Dr. med., Marburg

Jung, Norma, PD Dr. med., Köln

Kindermann, Ingrid, PD Dr. med., Homburg/Saar

Klemmer, Andreas, Dr. med., Marburg

Kluge, Stefan, Prof. Dr. med., Hamburg

Kochanek, Matthias, PD Dr. med., Köln

Köhler, Friedrich, Prof. Dr. med., Berlin

Lammert, Frank, Prof. Dr. med., Homburg/Saar

Ockenga, Johann, Prof. Dr. med., Bremen

Püllen, Rupert, PD Dr. med., Frankfurt

Schäfer, Jürgen, Univ. Prof. Dr. med., Marburg

Schmid, Sebastian, Prof. Dr. med., Lübeck

Schumm-Draeger, Petra-Maria, Prof. Dr. med., München

Schwarting, Andreas, Prof. Dr. med., Bad Kreuznach

Sieber, Cornel, Prof. Dr. med., Nürnberg/Regensburg

Spitzweg, Christine, Prof. Dr. med., München

Stallmach, Andreas, Prof. Dr. med., Jena

Strassburg, Christian, Prof. Dr. med., Hannover

Taube, Christian, Prof. Dr. med., Essen

Theobald, Matthias, Prof. Dr. med., Mainz

Watz, Henrik, PD Dr. med., Großhansdorf Wendtner, Clemens, Prof. Dr. med., München

Zeisberg, Michael, Prof. Dr. med., Göttingen 


\section{Young Investigator Award}

\section{Prävention und Rehabilitation}

\section{YIA01 \\ Genetic Risk Score is Associated With Prevalence of Advanced Neoplasms in a Colorectal Cancer Screening Population}

Korbinian Weigl'; Hauke Thomsen ${ }^{4}$; Yesilda Balavarca ${ }^{2}$; Jacklyn N. Hellwege $^{3}$; Martha J. Shrubsole ${ }^{3}$; Hermann Brenner ${ }^{1}$

${ }^{1}$ DKFZ Deutsches Krebsforschungszentrum, Klinische Epidemiologie und Alternsforschung, Heidelberg, Germany; ${ }^{2}$ NCT National Center for Tumor Diseases Heidelberg, Preventive Oncology, Heidelberg, Germany; ${ }^{3}$ Vanderbilt University School of Medicine, Division of Epidemiology, Nashville, United States; ${ }^{4}$ DKFZ Deutsches Krebsforschungszentrum, Division of Molecular Genetic Epidemiology, Heidelberg, Germany

Background \& Aims: The presence of specific single nucleotide polymorphisms (SNPs) can be used to calculate an individual's risk for colorectal cancer (CRC), called a genetic risk score (GRS). We investigated whether GRS can identify individuals with clinically relevant neoplasms in a screening colonoscopy population.

Methods: We derived a GRS based on 48 SNPs associated with CRC identified in a comprehensive literature search. We obtained genetic data from 1043 participants (50-79 years old) in a screening colonoscopy study in Germany, recruited from 2005 through 2013 (294 with advanced neoplasms, 249 with non-advanced adenomas, and 500 without neoplasms). Each participant was assigned a GRS by aggregating their risk alleles (0, 1, or 2). Risk of advanced neoplasms and non-advanced adenoma according to GRS was calculated by multiple logistic regression. Risk advancement periods were calculated. We replicated our findings using data from a subset of the Tennessee Colorectal Polyp Study.

Results: An increased GRS was associated with higher prevalence of advanced neoplasms, but not non-advanced adenomas. Participants in the middle and upper tertile of GRSs had a 2.2-fold and 2.7-fold increase in risk, respectively, of advanced neoplasms compared to those in the lower tertile. Adjusted odds ratios (ORs) were $1.09(95 \% \mathrm{Cl}$, 0.76-1.57) for non-advanced adenoma in the middle tertile and 1.05 ( $95 \% \mathrm{Cl}, 0.70-1.55)$ for non-advanced adenoma in the upper tertile. The ORs were largest for proximal advanced neoplasms for participants in the middle tertile $(\mathrm{OR}, 3.55 ; 95 \% \mathrm{Cl} 1.85-6.82)$ and the upper tertile (OR, $3.61 ; 95 \% \mathrm{Cl}$ 1.84-7.10). The risk advancement period for medium vs low GRS was 13.4 years ( $95 \% \mathrm{Cl}$ 4.8-22.0) and for high vs low GRS was 17.5 years $(95 \% \mathrm{Cl}, 7.8-27.3)$.
Conclusions: In a genetic analysis of participants in a CRC screening study in Germany, an increased GRS (based on CRC-associated SNPs) was associated with increased prevalence of advanced neoplasms. These findings might be used in defining risk-adapted screening ages.

Key words: colon cancer; cohort; variant; predisposition; prediction model

\section{Internistische Intensivmedizin}

\section{YIA02}

The gut incretin hormone GLP-2 is secreted from pancreatic alpha cells during acute inflammation and protects against sepsis

Florian Kahles'; Robert Werner Mertens ${ }^{4}$; Sebastian Diebold4; Julia Moellmann $^{4}$; Maria Chiara Arrivas ${ }^{4}$; Elias Haj-Yehia"; Darleen Sandoval' ${ }^{2}$; Lukas Martin ${ }^{5}$; Tobias Schürholz ${ }^{3}$; Jan Bruensing ${ }^{6}$; Alexander Koch ${ }^{6}$; Frank Tacke ${ }^{6}$; Corinna Lebherz ${ }^{4}$; Nikolaus Marx ${ }^{4}$; Michael Lehrke ${ }^{4}$

'Uniklinik RWTH Aachen, Medizinische Klinik I (Kardiologie), Aachen, Germany; ${ }^{2}$ University of Michigan, School of Public Health, Ann Arbor, United States; ${ }^{3}$ Universitätsmedizin Rostock, Klinik und Poliklinik für Anästhesiologie und Intensivtherapie, Rostock, Germany; ${ }^{4}$ Uniklinik RWTH Aachen, Medizinische Klinik ${ }^{1}$, Aachen, Germany ${ }^{5}$ Uniklinik RWTH Aachen, Klinik für Operative Intensivmedizin und Intermediate Care, Aachen, Germany; ${ }^{6}$ Uniklinik RWTH Aachen, Klinik für Gastroenterologie, Stoffwechselerkrankungen und Internistische Intensivmedizin, Aachen, Germany

Backround: The incretin hormone GLP-2 (glucagon-like peptide-2) is secreted by L-cells in the gut after food intake. GLP-2 enhances intestinal nutrient absorption and is clinically used for the treatment of patients with short bowel syndrom. Recently, GLP-2 has been found to be upregulated in patients with colitis with potentially protective effects through as of yet unknown mechanisms. The aim of this study was to characterize the role of GLP-2 during systemic inflammation.

Methods and Results: To analyze whether GLP-2 is modulated by the immune system, we first measured circulating GLP-2 levels in 223 critically ill patients with sepsis admitted to the ICU. Critically ill patients showed a 3.9 fold increase of total GLP-2 plasma levels in comparison to healthy controls. Moreover, GLP-2 levels were correlated with markers of inflammation (IL-6, PCT, CRP) and independently predicted mor- 
tality in critically ill patients. To elucidate the underlying mechanisms we injected $\mathrm{C}_{57 \mathrm{BL} / 6}$ mice with LPS or performed CLP (cecal ligation puncture; model for polymicrobial sepsis). Induction of lethal sepsis by CLP or LPS injection increased GLP-2 secretion. Further experiments in IL1R-/- and IL6-/- mice demonstrated that LPS-induced GLP-2 secretion is mediated by IL-6. To identify the source of GLP-2 secretion under inflammatory conditions, we injected LPS into Gcg-/- mice (preproglucagon; prescursor of GLP-2) with a tissue-specific reactivation of the Gcg gene in gut L-cells (GcgRA $\triangle$ vilCre) or pancretic alpha cells (GcgRA $\triangle P$ DX1-Cre). LPS-induced GLP-2 secretion was blocked in GcgRA $\triangle$ vilCre mice, while GcgRA $\triangle P D X_{1}-C r e$ mice showed a marked increase of GLP-2, indicating that inflammation-dependent GLP-2 production is derived from the pancreas and not from the gut. Finally, we analyzed whether inflammatory upregulation of GLP-2 has functional immunomodulatory relevance and adminstered GLP-2 (1-33) or saline as control per central jugular vein catheter mice who underwent CLP. GLP-2 treatment improved LV-contractility (dp/dtmax) in septic cardiomyopathy, clinical sepsis score and inhibited sepsis-induced hypotension. Mechanistically GLP-2 reduced myeloid immune cell infiltration into heart and liver and decreased proinflammatory cytokine levels in various organs and the blood (TNF-a, IL-6, IL-1ß). After broad GLP-2 receptor profiling we found maximum mRNA expression in gut tissues with no expression on immune cells. By further mechanistic studies we found GLP-2 to protect sepsis-induced gut barrier dysfunction.

Conclusion: By using a translational approach (clinical study, experimental in vivo and in vitro studies) we identified that acute inflammation activates pancreatic GLP-2 secretion independent of food intake. Inflammation-induced GLP-2 activation protects against sepsis and its related organ dysfunctions. GLP-2 provides a previously unknown link between the pancreas and the immune system, which might open new avenues for the treatment of patients with sepsis.

\section{Innere Medizin integral}

\section{YIA03 \\ Negativer Effekt von Protonenpumpeninhibitoren bei Leberzirrhose ist abhängig von häufigen Genvarianten}

Christina Schneider'; Roman Liebe'; Edith Vandieken'; Markus Casper'; Beate Appenrodt'; Robin Greinert ${ }^{2}$; Alexander Zipprich ${ }^{2}$; Cristina Ripoll ${ }^{2}$; Frank Lammert'; Matthias Reichert ${ }^{1}$

'Universitätsklinikum des Saarlandes, Homburg, Innere Medizin II, Homburg, Germany; ${ }^{2}$ Martin-Luther-Universität Halle-Wittenberg, Innere Medizin I, Halle, Germany

Hintergrund: Protonenpumpeninhibitoren (PPI) begünstigen infektiöse Komplikationen und erhöhen möglicherweise die Mortalität von Patienten mit Leberzirrhose. Häufige Risikovarianten in den $\mathrm{Pa}$ tatin-like Phospholipase Protein 3 (PNPLA3)- und Membrane-bound O-acyltransferase domain-containing protein 7 (MBOAT7) Genen tragen zur Progression chronischer Leberkrankheiten bei. Daher haben wir den Einfluss der PPI-Einnahme auf Überleben und Entstehung systemischer Komplikationen bei Leberzirrhose in Abhängigkeit vom Vorhandensein genetischer Risikovarianten untersucht.

Patienten und Methoden: Insgesamt wurden 998 Patienten aus den Universitätskliniken in Homburg und Halle zwischen 2014 und 2017 eingeschlossen. Die PPI-Einnahme wurde als tägliche, dauerhaft eingenommene Dosis von $\geq 20 \mathrm{mg}$ Pantoprazol (oder eine äquivalente Dosis eines anderen PPI) definiert. Nach der Genotypisierung für die Risikovarianten rs738409 (PNPLA3) und rs641738 (MBOAT7) wurden das transplantionsfreie Überleben sowie das Auf- treten von Komplikationen prospektiv erfasst, und nach dem Vorhandensein der Risikovarianten stratifiziert.

Ergebnisse: Insgesamt wurden 700 Patienten mit PPI Einnahme (70\%) eingeschlossen. Das mediane Alter lag bei $61(53-68)$ Jahren, der mediane MELD Score bei 11 (8 - 15). Die Zirrhose war bei 537 (54\%) alkoholischer, bei 166 (17\%) viraler und bei 295 (29\%) anderer Genese. Von diesen Patienten erhielten 94\% Pantoprazol (63\% 40 mg, 30\% 80 $\mathrm{mg}$ und $7 \% 20 \mathrm{mg}$ p.d.). In der Überlebenszeitanalyse nach Kaplan Meier war das Überleben bei PPI Einnahme bei Patienten ohne PNPLA3-Risikovariante verkürzt (Log rank $p=0.005$ ), wohingegen bei Patienten mit Nachweis einer Risikovariante in PNPLA3 keine signifikant verkürzte transplantatfreie Überlebenszeit vorlag (Log rank $p=0.02$ ). In der univariaten Cox-Regression waren weibliches Geschlecht, Bilirubin, Kreatinin, INR, PPI und alkoholische Lebererkrankung unabhängige Prädiktoren für das transplantatfreie Überleben. In der multivariaten Coxregression verblieben bei den Trägern der Wildtyp-Allele PPI (adjustierte Hazard Ratio [aHR] 0,48; 95\% Konfidenzintervall [CI] $0,23-0,96 ; p=0,039)$ und MELD-Score (aHR 1,17; 95\% Cl 1,13-1,22; $p<$ $0,001)$, bei den Trägern der PNPLA3-Risikovariante nur der MELD-Score (aHR 1,16; 95\% Cl 1,13-1,19; p<0,001) als unabhängige Prädiktoren. Für alle Endpunkte wurden für MBOAT7-Varianten keine Unterschiede gefunden. Unter den PPI-assoziierten Komplikationen ergab sich nur für bakterielle Infektionen ein ähnliches Risiko bei Patienten mit (Odds ratio [OR] 2,20; 95\% Cl 1,23 - 3,93; $\mathrm{p}=0,007)$ und ohne (OR 2,23; $95 \% \mathrm{Cl}$ 1,23-4,05; $\mathrm{p}=0,007$ ) PNPLA3-Risikoallel.

Schlussfolgerungen: In dieser prospektiven Kohorte zeigte sich nur bei Patienten ohne PNPLA3- Risikovariante ein geringeres transplantatfreies Überleben, jedoch nicht bei Trägern der Mutation p.1148M. Mechanistische Untersuchungen dieser Gen-Umwelt-Interaktion sind nun erforderlich.

\section{Pneumologie}

\section{YIA04}

\section{Adherence to respiratory and non-respiratory medication in} patients with COPD: results of the German COSYCONET cohort

Kathrin Kahnert'; Norbert Königsdorfer ${ }^{9}$; Sandra Söhler ${ }^{2}$; Tobias Welte ${ }^{3}$; Jürgen Behr ${ }^{1}$; Joachim H. Ficker ${ }^{4}$; Robert Bals ${ }^{5}$; Henrik Watz ${ }^{6}$; Johanna Lutter $^{7}$; Tanja Lucke ${ }^{9}$; Frank Biertz ${ }^{10}$; Peter Alter ${ }^{8}$; Claus F. Vogelmeier ${ }^{8}$; Rudolf A. Jörres ${ }^{9}$

'Klinikum der Universität München, Medizinsiche Klinik V, München, Germany; ${ }^{2}$ Klinikum der Universität Marburg, ASCONET Study Coordination Office, Marburg, Germany; ${ }^{3}$ Hannover Medical School, Department of Pneumology, Hannover, Germany; ${ }^{4}$ General Hospital Nuernberg, Department of Respiratory Medicine, Nürnberg, Guernsey; ${ }^{5}$ Saarland University Hospital, Department of Internal Medicine V, Homburg, Germany; ${ }^{6}$ Pulmonary Research Institute at LungenClinic Grosshansdorf, Airway Research Center North, Grosshansdorf, Germany; ${ }^{7}$ Helmholtz Zentrum München $\mathrm{GmbH}$, Institute of Health Economics and Health Care Management, Neuherberg, Germany; ${ }^{8}$ Philipps-University Marburg, Department of Medicine, Pulmonary and Critical Care Medicine, University Medical Center Giessen and Marburg, Marburg, Germany; ${ }^{9}$ Klinikum der Universität München, Institute and Outpatient Clinic for Occupational, Social and Environmental Medicine, München, Germany; ${ }^{10}$ Hannover Medical School, ${ }^{9}$ Institute for Biostatistics, Hannover, Ghana

Adherence to COPD medication is often considered to be lower than in other chronic diseases. To achieve comparability we analysed the intake of all medication in the same patients with COPD and frequent comorbidities. 
Within the COPD cohort COSYCONET we contacted 1042 patients, mailing them a list with all medication regarding all their diseases, asking for regular, irregular and non-intake.

Valid responses were obtained in 809 patients covering a wide spectrum of drugs. Intake of LABA, LAMA or ICS was regular in $90.9 \%$ of patients, even higher for cardiovascular and anti-diabetes medication but lower for hyperlipidemia and depression/anxiety medication. Regular intake of respiratory medication did not depend on GOLD groups A-D or grades 1-4, highest in patients with concomitant cardiovascular disorders and lowest for concomitant asthma. It was slightly larger for LAMA and LABA administered via combined compared to single inhalers, and lower when similar compounds were prescribed twice. Most differences did not reach statistical significance owing to the overall high adherence.

Our results indicate a high adherence to respiratory medication in participants of a COPD cohort, especially in those with cardiovascular comorbidities. Compared to data from other studies, this observation suggests room for improvement of adherence, possibly through educational strategies.

\section{Digitale Medizin}

\section{YIA05 \\ How to prevent effectively and durable medical errors by an electronic, central, interdisciplinary chemotherapy (CTx) management system}

Amelie Rösner'; Stefanie Ajayi²; Heike Reinhardt ${ }^{2}$; Petra Schilli'; Markus Ruch'; Günther Storz'; Beate Rautenberg ${ }^{3}$; Henning Schäfer'; Frank Meiß ${ }^{5}$; Martin J. Hug'; Justus Duyster'; Monika Engelhardt ${ }^{1}$

'University Medical Center Freiburg, Department of Hematology and Oncology; Comprehensive Cancer Center Freiburg (CCCF), Freiburg, Germany; ${ }^{2}$ University Medical Center Freiburg, Department of Hematology and Oncology, Freiburg, Germany; ${ }^{3}$ University Medical Center Freiburg, Department of Gynaecology and Obstetrics, Freiburg, Germany; ${ }^{4}$ University Medical Center Freiburg, Department of Radiooncology, Freiburg, Germany; ${ }^{5}$ University Medical Center Freiburg, Department of Dermatology, Freiburg, Germany; University Medical Center Freiburg, Pharmacy, Freiburg, Germany;

Introduction: Patients (pts) with cancer often receive complex medication regimens. This may lead to high medication error (ME) rates[1,2], which can have an impact on patients' clinical outcome. Our central CTx Surveillance System (CSS) within the Comprehensive Cancer Center Freiburg (CCCF) incorporates all important elements to avoid ME, namely a) detailed CTx protocols including co-medication, b) SOPs and clinical pathways, c) a multidisciplinary team, d) an electronic CTx ordering system. The electronic CTx management tool (eCTxMT) - ChemoCompile (CC) - has become an indispensable part of the clinical routine. We describe the impressive reduction of ME and practical targets for clinical intervention.

Methods \& Results: CC is a web-based database application containing $>600$ standardized, regularly updated CTx protocols (partly published in: Das Blaue Buch[3]). Physicians generate CTx orders via $\mathrm{CC}$, which are processed by the clinical pharmacy (CP) and our Clinical Cancer Research Group (CCRG). Any detected error is instantly reported to the responsible physician and consequently corrected. If a CTx order is flawless, a detailed pt-individual treatment schedule created via CC is sent to the ward and used as a valid prescription. Our eCTxMT was first implemented at the department of Hematology \& Oncology and has meanwhile been transferred to all other adult oncology units of the CCCF and external institutions (via CC).

We analyzed all CTx orders between 2005 and 2017 concerning their rate of MEs. In 2005 the error rate analysed was $4.2 \%$. Subsequently after implementation of eCTXMT at our center in 2006 the error rate decreased and remaind stable over the years at $2 \%$ (2017: 1.8\%). Of notice, all of these errors were efficiently avoided. This considerable improvement is a result of the implementation of a) an eCTxMT, b) the triple control system by CCRG, CP and physicians, c) continuous education and d) the Blue Book[3]. Due to our CSS, $>99.9 \%$ of all MEs were detected and eliminated, thus did not reach the pt[1].

According to our recent analysis, $\sim 60 \%$ of detected and avoided MEs could be prevented by further IT-development of the eCTXMT[4].

In 2018 we implemented our new upgraded error analysis dividing the prescription errors into administrative and medical errors. Administrative errors result in necessary time consumption without impact on pt's safety, in contrary to medical errors.

Conclusions: Our central CTx management system has shown to substantially improve the quality of CTx treatment and pt safety, providing work simplification for physicians and nurses. CC as essential part of the CSS is also available to external cancer centers. This generates benefits for pts, healthcare staff, CP and pharmaceutical companies. Despite the great improvement potential for eCTXMT, $30-40 \%$ of errors are not avoidable electronically, therefore the CCRG, ward pharmacists or other surveillance teams remain indispensable for entirely safe CTx prescribing.

[1] Markert A, Thierry V, Kleber M, Behrens M, Engelhardt M. Chemotherapy safety and severe adverse events in cancer patients: strategies to efficiently avoid chemotherapy errors in in- and outpatient treatment. Int. J. Cancer. 2009;124(3):722-728

[2] Walsh KE et al. Medication errors among adults and children with cancer in the outpatient setting. JCO.2009; 27(6):891-6

[3] Engelhardt, Berger, Duyster, Mertelsmann: Das Blaue Buch, 6th Edition, Springer, 2016

[4] Reinhardt $\mathrm{H}$, ...Engelhardt M. Avoiding chemotherapy prescribing errors: analysis and innovative strategies. Cancer. 2018 in press

\section{Innere Medizin integral}

\section{YIA06}

\section{Regulation der p53-Familie und des Zelltodes in Darm-} epithelzellen als Mechanismus der Pathogenese der spontan bakteriellen Peritonitis

Annika Scholtis'; Marika Haderer'2 Elisabeth Aschenbrenner'2; Heidi Gschwendtner2; Claudia Kunst²; Karsten Gülow²; Martina Müller-Schilling ${ }^{2}$

'Universitätsklinikum Regensburg, Innere Medizin I, Straubing, Germany; 2Universitätsklinikum Regensburg, Klinik und Poliklinik für Innere Medizin I, Gastroenterologie, Hepatologie, Endokrinologie, Rheumatologie und Infektiologie, Regensburg, Germany

Hintergrund: Die spontan bakterielle Peritonitis (SBP) ist eine Infektion der Aszitesflüssigkeit ohne erkennbare Infektionsquelle. Sie ist eine der häufigsten Komplikationen von Patienten mit Leberzirrhose und Aszites. Die SBP zeigt eine sehr hohe Ein-Jahres-Mortalität von 
bis zu 93\%. Bis heute sind die genauen Pathomechanismen der SBP ungeklärt, doch man geht von einer bakteriellen Translokation aus dem Darmlumen in den Aszites aus. Die p53-Familie ist besonders für ihre tumorsuppressive Wirkung bekannt, doch sie spielt auch bei bakteriellen Infektionen eine Rolle, z.B. durch die Hemmung einer Entzündungsantwort. Um die Interaktion von Bakterien mit Darmepithelzellen besser zu verstehen, wurde die Regulation von p53 sowie die Induktion von Zelltod nach bakterieller Stimulation untersucht.

Methoden: Die humane intestinale Epithelzelllinie HCT-116wt exprimiert wildtyp-p53. Sie wurde mit Escherichia coli (E.coli) in den Konzentrationen MOlo bis MOl5 für 2 oder 4 Stunden koinkubiert. Um die Effekte von bakteriellen Bestandteilen und Metaboliten von denen kompletter Bakterien zu unterscheiden, wurden die Zellen auch mit E.coli Lipopolysaccharid (LPS) inkubiert und Transwell-Experimente durchgeführt, bei denen die Zellen und Bakterien durch eine semipermeable Membran getrennt waren. Das Proteinniveau der p53-Familie wurde mittels Western Blot analysiert. Der Zelltod wurde durchflusszytometrisch untersucht, wobei die Zellen vor Bakterienzugabe mit verschiedenen Zelltod-Inhibitoren (zVAD-FMK, Necrostatin-1, Ferrostatin-1), Antioxidantien (Trolox, NAC), Cyclophilin-Inhibtoren (Ciclosporin A) und Metallchelatoren (BAPTA-AM, 2,2区-Bipyridyl) behandelt wurden.

Ergebnisse: Koinkubation von HCT-116wt und E.coli bewirkte eine zeit- und dosisabhängige Abnahme der Proteinlevel von p53 und p73. Auch die Stimulation mit LPS zeigte ähnliche reduzierende Effekte auf die p53-Familie. Im Gegensatz dazu wurden im Transwell-Experiment die Proteinlevel nicht beeinflusst und auch hitzeinaktivierte Bakterien zeigten keinen Effekt. Trotz reduziertem p53-Level resultierte eine Behandlung mit E.coli in hohen Zelltod-Raten. Hitzeinaktivierte Bakterien und LPS lösten keinen Zelltod aus. Der Zelltod konnte durch zVAD-FMK, Necrostatin-1, Ferrostatin-1, Trolox, NAC, Ciclosporin oder BAPTA-AM nicht blockiert werden. Der Eisenchelator 2,2区-Bipyridyl führte hingegen zu verminderten Zelltod-Raten ( $56 \%$ vs. $75 \%$ ).

Schlussfolgerung: Direkter Kontakt von Darmepithelzellen und vitalen Bakterien resultiert in einer Herabregulation der p53-Familie. Trotz vermindertem p53-Spiegel löst E. coli jedoch einen nicht apoptotischen, aber möglicherweise Eisen-abhängigen Zelltod in HCT-116wt Zellen aus. Im Rahmen einer Leberzirrhose kann dies einen bakteriellen Mechanismus darstellen, um sich vor der Immunabwehr zu schützen und in der Folge die intestinale Barriere zu überwinden und so eine SBP auszulösen.

\section{Seltene Erkrankungen}

\section{YIA07 \\ Desminopathien - Desmin-Mutationen als Ursache von familiären Kardiomyopathien, Arrhythmien und plötzlichen Herztod}

Björn Fischer'; Sven Dittmann'; Andreas Brodehl'; Anne Kayser'; Hendrik Milting ${ }^{2}$; Eric Schulze-Bahr ${ }^{1}$

${ }^{1}$ Universitätsklinikum Münster, Institut für Genetik von Herzerkrankungen (IfGH), Münster, Germany; ${ }^{2}$ Herz- und Diabeteszentrum NRW, Erich und Hanna Klessmann-Institut, Bad Oeynhausen, Germany

Einleitung: Die Desminopathie ist eine klinisch heterogene, genetische Erkrankung an der Schnittstelle zwischen Kardiologie und Neurologie, manifest als diverse Myopathie- und Kardiomyopathieformen. Desminopathien sind mit einer Prävalenz $<$ 1:2.000 insgesamt seltene Erkrankungen. Ursächlich sind meist nicht-synonyme, hetero- zygote Mutationen im DES-Gen, welches für das Intermediärfilamentprotein Desmin kodiert. Material und Methoden: In zwei Familien mit einer dilatativen Kardiomyopathie (DCM) wurde jeweils eine neue DES-Genmutation mittels Next-Generation-Sequencing (NGS) identifiziert und weiter molekularbiologisch und zellbiologisch charakterisiert. Es erfolgte eine bioinformatische Bewertung der DES-Varianten und eine Klassifikation nach den Kriterien des American College of Genetic and Genomic Medicine (ACMG). Zur funktionellen Charakterisierung wurden DES-Mutationen generiert und in das pm-Ruby-N1Desmin Plasmid inseriert und anschließend in $\mathrm{H} 9 \mathrm{C}_{2}$ - und SW-13-Zellen transfiziert. Zudem wurde verfügbares, explantiertes Myokard-Gewebe eines Mutationsträgers immunhistologisch untersucht. Ergebnisse: Die Sequenzierung zeigte zwei neue Mutationen im DES-Gen: p.lle402Thr (c.1205T>C, Familie I) und p.Glu410Lys (c. 1228G>A, Familie II), die in großen Kontrollpopulationen (gnomAD, EVS) komplett abwesend sind und in allen Pathogenitäts-Vorhersageprogrammen kohärent als pathologisch bewertet wurden. Entsprechend der ACMG handelt es sich um, pathogenic variants' (class V). Eine Zweitmutation wurde nicht identifiziert. Klinisch zeigte der Indexpatient der Familie I eine milde DCM und eine Myopathie. In der großen Familie II hingegen zeigte sich eine schwere, familiäre DCM, eine arrhythmogene rechtsventrikuläre Kardiomyopathie (ARVC), diverse, zum Teil maligne Herzrhythmusstörungen und multiple plötzliche Herztode. In in-vitro-Versuchen zeigten beide Mutationen pathologische Desmin-Aggregate, wohingegen der Wildtyp reguläre Filamente ausbildete. Die Desmin-Aggregate wurden zudem in der explantierten Herzprobe aus Familie II nachgewiesen. Schlussfolgerung: Die Ergebnisse zeigen, dass Desminopathien eine variable, kardiale Expressivität haben können, die zum Teil schwer ausgeprägt sein kann. Die Bildung vermutlich toxischer Proteinaggregate untermauert die Pathogenitätsvorhersagen. Die frühzeitige Erkennung von DES-Mutationen hilft bei der genetische Beratung und Therapie betroffener Familien.

\section{YIA08 \\ Inhibition von CD38 zeigt anti-leukämische Wirksamkeit in der akuten myeloischen Leukämie}

Meike Farber; Lucas Arnold; Yiyang Chen; Michael Möllmann; Alexander Carpinteiro; Ulrich Dührsen; Maher Hanoun

Universitätsklinikum Essen, Hämatologie, Essen, Germany

Die akute myeloische Leukämie (AML) ist gekennzeichnet durch ein frühes Versagen der gesunden Hämatopoese und eine hohe Rezidivrate, die auf persistierenden Leukämiezellen in der Knochenmarknische beruht. Die hämatopoetische Stammzellnische zeigt infolge der Leukämieinfiltration bedeutende strukturelle und funktionelle Veränderungen mit einer beeinträchtigten immunologischen Kontrolle. $\mathrm{CD}_{3} 8$ ist ein Glykoprotein, das auf immunregulatorischen Zellen und häufig auf AML-Blasten exprimiert wird und dessen Inhibition mit dem monoklonalen Antikörper Daratumumab bereits hohe Wirkung in der Therapie des Multiplen Myeloms gezeigt hat. Um die anti-leukämische Wirksamkeit der CD38-Inhibition und deren Einfluss auf das leukämische Mikroenvironment in der AML zu untersuchen, haben wir ein 3D-in vitro Modell mit humanen Endothelzellen und mesenchymalen Stammzellen etabliert, worin primäre AML- oder gesunde mononukleäre Zellen kultiviert werden. Die Zugabe von Daratumumab zu monokultivierten AML-Zellen reduzierte das Zellwachstum um nur 7,2\% ( $p<0,01)$, während die anti-leukämische Wirksamkeit in der Triple-Kultur auf 35,6\% anstieg $(p<0,05)$. Dieser Effekt von Daratumumab in der AML hing nicht von der Höhe der $C_{3}$ 38-Expression $\mathrm{ab}$, ebenso zeigte sich kein relevanter zellautonomer Einfluss auf die Proliferation oder Apoptose der AML-Zellen. Angesichts der bedeutenden immunologischen Effekte von Daratumumab führten wir Effektorfunktionsassays durch. Während die $C_{3}$ 38-Inhibition keine Antikörper-abhängige zelluläre Zytotoxizität (ADCC) oder Komple- 
ment-abhängige Zytotoxizität (CDC) zeigte, beobachteten wir eine signifikante Induktion der Antikörper-abhängigen Phagozytose (ADCP) durch Daratumumab. Humane Makrophagen zeigten eine $\sim 1,7 \mathrm{x}$ gesteigerte Phagozytose von AML-Zelllinien und primären AML-Zellen im Vergleich zur lgG1-Kontrolle $(p<0,05)$.Um die anti-leukämische Aktivität von Daratumumab in vivo nachzuweisen, transplantierten wir primäre humane AML-Zellen intravenös in immundefiziente NODscid-gamma-Mäuse. Leukämische Mäuse wurden über 4 Wochen mit all-trans Retinsäure (ATRA), was die CD38-Expression auf AML-Zellen signifikant hochregulierte und einmal wöchentlich mit Daratumumab oder IgG1 behandelt. Eine Monotherapie mit Daratumumab zeigte eine geringfügige Wirksamkeit in vivo. Auffällig war eine Abnahme der Leukämielast im peripheren Blut ( 39\%), wogegen sich im Knochenmark und in der Milz keine signifikanten Unterschiede zeigten ( $n=12-14$ pro Gruppe). In der Tat zeigten in vivo Homing-Experimente, dass Daratumumab die Zirkulation von AML-Zellen signifikant beeinträchtigt. Zusammenfassend zeigt die Inhibition von $\mathrm{CD}_{38}$ eine anti-leukämische Aktivität in der AML, welche vor allem durch das Mikroenvironment vermittelt ist. In weiteren Experimenten soll die anti-leukämische Wirksamkeit von Daratumumab kombiniert mit niedrig dosierter Chemotherapie untersucht werden, um ggf. einen neuen, gegen das leukämische Mikroenvironment gerichteten Therapieansatz zu etablieren.

\section{Multimorbidität und Polypharmazie}

\section{YIA09}

\section{Bedeutung der Bestimmung von Anti-Drug-Antibodies bei der Therapie von chronisch entzündlichen Darmerkrankungen mit Infliximab.}

\author{
Anna Langmann ${ }^{1,2}$; Peter Langmann \\ ${ }^{2}$ Institut für Klinische Chemie und Pathobiochemie der Technischen \\ Universität München, Germany; ${ }^{1}$ Praxis für Gastroenterologie, Gastroen- \\ terologie, Karlstadt, Germany
}

Einleitung und Zielsetzung: In der Therapie chronisch entzündlicher Darmerkrankungen ist Infliximab (IFX) neben weiteren Biologika ein wichtiger Bestandteil. Ein Problem ist die Entwicklung von Anti-Drug-Antibodies (ADA), welche die Pharmakokinetik und die Effektivität der eingesetzten Substanz negativ beeinflussen.

Hier werden Daten des Therapeutischen Drug Monitorings (TDM) eines Kollektives von 57 Patienten ( $n=47$ Morbus Crohn; $n=10$ Colitis ulcerosa) retrospektiv ausgewertet, die Entwicklung von ADA im Therapieverlauf, der Einfluss der ADA auf die IFX-Drug-level und das Erzielen und Aufrechterhalten einer klinischen Remission untersucht.

Methode: Es erfolgt die Bestimmung des IFX trough-levels zeitgleich mit dem ADA - Spiegel vor der nächsten Infusion. Die Analyse erfolgt mittels kommerziell verfügbarer Testsysteme (IDKmonitor ${ }^{\oplus i n f l i x i m a b}$ drug level ELISA und total ADA Elisa). Eine Auswertung des ADA-Titers erfolgt in Korrelation mit dem IFX-Drug level, begleitender immunsuppressiver Therapie mit Azathioprin (AZA) und dem klinischen Ansprechen.

Ergebnisse und Schlussfolgerung: Bei $n=57$ Patienten wurden 686 Analysen durchgeführt. $\mathrm{N}=49$ Patienten wurden mit IFX behandelt $(n=15 \mathrm{IFX}+\mathrm{AZA})$, Adalimumab erhielten $25 \%$, Vedoluzimab $12 \%$, Golimumab $4 \%$ und Ustekinumab $4 \%$ der Patienten. IFX-Drug-level lagen bei $6,30+/-5,68 \mu \mathrm{g} / \mathrm{ml}$, IFX-ADA $>10 \mu \mathrm{g} / \mathrm{ml}$ waren bei $\mathrm{n}=17$ (35\%) Patienten (IFX 15/34 (44\%); IFX+AZA 2/15 (13\%) nachweisbar. Die ADA-Titer lagen bei $147,72+/-174,70 \mu \mathrm{g} / \mathrm{ml}$. Dies führte durchschnittlich nach 6,9
Monaten bei $83 \%$ der Patienten zum Therapieabbruch. Niedrig-titrige IFX- ADA < 10 $\mu \mathrm{g} / \mathrm{ml}$ waren bei $27 / 49$ (55\%) (IFX 14/34 (41\%);( IFX+AZA

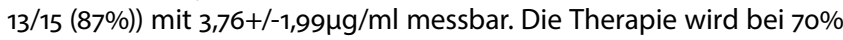
der Patienten seit 12,1 Monaten fortgesetzt.

Exemplarisch wird der Verlauf von Patienten mit niedrig-titrigen ADA unter einer Dosiseskalation gezeigt.

Somit zeigt sich, dass sehr häufig ADA in niedrigem Titer nachweisbar sind. Eine remissionerhaltende Therapie, ggf. unter einer Dosisanpassung, aber möglich ist.

Das TDM ist essentieller Bestandteil des Therapiemanagements einer Biologikatherapie bei CED. Besondere Bedeutung hat die ADA-Bestimmung. Wesentlich ist zukünftig, auch für neue Substanzen, die Differenzierung zwischen neutralisierenden und nichtneutralisierenden Antikörpern, sowie sensitivere Bestimmungsmöglichkeiten von niedrig-affinen und niedrig-titrigen Antikörpern. Dies unterstreicht die Bedeutung der Entwicklung neuer genauerer Messsysteme neben den konventionellen Elisa- Verfahren, zum Beispiel mittels Biosensoren. Somit wird eine individuell gesteuerte Dosis des Medikaments im Rahmen eines TDM ermöglicht.

[1] Moussa EM, et al. Immunogenicity of therapeutic protein aggregates. J Pharm Sci 2016:105:417-430

[2] Strand V, et al. Immunogenicity of biologics in chronic inflammatory diseases: a systematic review. BioDrugs 2017;31:299-316 


\section{Digitale Medizin}

\section{PS001}

\section{Telemonitoring im ländlichen Raum - Akzeptanz und Einfluss auf das Verhalten der Patienten}

Bettina Zippel-Schultz; ; Frauke Wenzelburger ${ }^{2}$; Thomas Luiz ${ }^{3}$; Lena Sembach $^{3}$; Rainer Zotz ${ }^{4}$; Burghard Schumacher ${ }^{2}$; Thomas M. Helms ${ }^{5}$

'Deutsche Stiftung für chronisch Kranke, Innovationsmanagement, Berlin, Germany; ${ }^{2}$ Westpfalz-Klinikum, Klinik für Innere Medizin ${ }^{2}$, Kaiserslautern, Germany; ${ }^{3}$ Fraunhofer-Institut für Experimentelles Software Engineering IESE, Process Engineering, Kaiserslautern, Germany; ${ }^{4}$ Marienhaus Klinikum Eifel-Bitburg, Innere Medizin I - Kardiologie und Angiologie, Bitburg, Germany; ${ }^{5}$ Deutsche Stiftung für chronisch Kranke, Vorstand, Berlin, Germany

Ländliche Regionen stehen einem zunehmenden Ungleichgewicht zwischen dem sinkenden Angebot und der steigenden Nachfrage nach Gesundheitsleistungen gegenüber. Telemonitoring als Ausdruck der digitalen Transformation des Gesundheitswesens birgt die Chance, diesen wachsenden Herausforderungen zu begegnen. Die Akzeptanz durch die Patienten und der Einfluss auf das Verhalten der Patienten sind zentrale Erfolgsfaktoren, die die nachhaltige Etablierung von Telemonitoring mitbestimmen werden. Im Telemonitoring-Projekt EHeR $\cdot$ versorgt wurden im Eifelkreis Bitburg-Prüm 43 Patienten $(72,3+/-10,1$ Jahre alt) mit Herzinsuffizienz (EF < 45\%, NYHA II-III) über 6 Monate betreut. Die Patienten erhielten Bluetooth-fähige Geräte: Waage und Blutdruckmessgerät. Die gemessenen Daten wurden über eine gesicherte Internetverbindung verschlüsselt in die elektronische Fallakte des Telemedizinzentrums übertragen. Notwendige medizinische Maßnahmen wurden angestoßen, sobald individuell definierte Grenzwerte überschritten wurden. Klare Prozesse regelten die Kontakte der ausgebildeten Schwestern des Telemedizinzentrums mit den Patienten und weiteren beteiligten Ärzten. In den regelmäßigen, teil-standardisierten Telefonaten wurden z. B. Symptome, Arztbesuche und Therapietreue erfasst. Als primärer Endpunkt wurde der 6 Minuten-Gehtest zu Beginn und nach 6 Monaten durchgeführt. Die Zufriedenheit und Akzeptanz wurde anhand der validierten Fragebögen TeSS (Telehealth Satisfaction Scale), TSG (Telemedicine Satisfaction Questionaire) und SeCu-16 erhoben. EHeR •versorgt verzeichnete 4 drop-outs (1 Tod, 2 Zunahmen von Demenz, 1 Abbruch). Der positive Einfluss des Telemonitorings zeigte sich in der signifikanten Steigerung der Leistungsfähigkeit: 6 Minuten-Gehtest zu Beginn to $=370.8 \mathrm{~m}$ $+/-102.1 \mathrm{~m}$ und nach 6 Monaten $\mathrm{t} 1=446.0 \mathrm{~m}+/-133.2 \mathrm{~m}, \mathrm{p}=0,002, \mathrm{n}=30$. Die vornehmend älteren Patienten standen technischen Neuerungen generell eher skeptisch gegenüber. Trotzdem war die Zufriedenheit mit EHeR•versorgt sehr hoch. Das Betreuungsprogramm lenkte die Aufmerksamkeit der Patienten auf ihre Erkrankung. Die edukativen Elemente steigerten das Wissen und stärkten die Patienten im Umgang mit der Herzinsuffizienz. Die Patienten fühlten sich deutlich selbstständiger als zuvor. Das Telemonitoring gab ihnen eine hohe Sicherheit im Alltag, im Beruf und bei Freizeitaktivitäten. Generell gaben die Patienten an, dass sie bereit sind, Telemonitoring als Teil der Gesundheitsversorgung zu akzeptieren. Sie sehen die Vorteile, z. B. die Vermeidung von Wegen und Wartezeiten. Allerdings waren sich die Patienten nicht sicher, wie sehr die im Telemonitoring gewonnenen Daten wirklich in der Behandlungsstrategie ihres (Haus-)Arztes berücksichtigt wurden. Hier bedarf es zukünftig weiterer Anstrengungen, die niedergelassene Ärzteschaft mehr in das Telemonitoring einzubinden und sowohl organisatorische als auch finanzielle Lösungen zu schaffen, die eine nachhaltige und flächendeckende Etablierung von Telemonitoring gewährleisten.

\section{PSO02}

\section{Klinische Entscheidungsunterstützung durch Künstliche Intelligenz}

Laura Zwack'; Yvonne Weber²; Christoph Sippel³; Gökhan Günyak

${ }^{1}$ Elsevier GmbH, Clinical Solutions DACH + EE, München, Germany; ${ }^{2}$ Universitätsklinikum Tübingen, Hertie Institut für Klinische Hirnforschung, Tübingen, Germany; ${ }^{3}$ Johanniter-Krankenhaus Bonn, Internistische Onkologie, Bonn, Germany; ${ }^{4}$ Robert-Bosch-Krankenhaus, IT, Stuttgart, Germany

Clinical Decision Support Systeme (CDSS) werden als wichtige Waffe im Kampf gegen vermeidbare medizinische Fehler angesehen. Zudem können sie die Umsetzung von evidenzbasiertem Wissen in der Praxis beschleunigen. Im Zentrum effektiver CDSS stehen Künstliche Intelligenz (KI) und evidenzbasierte Medizin (EBM). In unserem Vortrag unterscheiden wir die verschiedenen Formen von KI und diskutieren sie im Hinblick auf ihre Eignung für den Einsatz in CDSS. Zudem zeigen wir, wie ein Expertensystem basierend auf deklarativer Künstlicher Intelligenz die Diagnostik und Therapie komplexer Erkrankungen wie Epilepsie, Kolonkarzinoms sowie der Stammzelltransplantation unterstützen kann.

Bei Clinical Decision Support geht es um den Einsatz von schwacher $\mathrm{Kl}$, d.h. ärztliche Entscheidungen sollen in Einzelbereichen unterstützt werden. Aus dem Bereich der Schwachen KI bieten sich insbesondere Wissensbasierte Systeme sowie Machine Learning zum Einsatz in CDSS an. Wissensbasierte Systeme modellieren eine Form rationaler Intelligenz für sogenannte Expertensysteme. Diese sind in der Lage, auf eine Frage des Anwenders auf Grundlage formalisierten Fachwissens und daraus gezogener logischer Schlüsse Antworten zu liefern. Machine-Learning ist für die CDSS nicht immer geeignet. Die heutigen „Big Data“ über die klinische Versorgung repräsentieren weniger als 20 Prozent der evidenzbasierten Erkenntnisse. Zudem können Erhebungsverzerrungen erhebliche Auswirkungen haben. So sind Frauen, ethnischen Minderheiten und älteren Menschen in der Diagnostik und Therapie vieler Krankheiten unterrepräsentiert. Machine Learning auf Basis dieser Daten würde zu falschen Empfehlungen kommen.

Wir stellen daher ein Clinical Decision Support Framework vor, das einen deklarativen Ansatz der Wissensrepräsentation nutzt. Das Spezialwissen von evidenzbasierten Leitlinien und die Schlussfolgerungsfähigkeit klinischer Experten werden in der Programmiersprache PROforma formal nachgebildet, so dass sie von einem Computer interpretiert werden können. Der Lösungsweg wird nicht algorithmisch vorgegeben wird, sondern es werden nur mehr die Bedingungen definiert, die die Lösung des Problems erfüllen soll. Dies ermöglicht es zu überprüfen, wie Entscheidungen getroffen wurden oder warum bestimmte Optionen empfohlen wurden und andere nicht - es handelt sich also nicht um eine „Black-box" wie bei Machine Learning.

Wir zeigen die Ergebnisse der ersten klinischen Evaluationen des Clinical Decision Support Frameworks in drei Anwendungsfällen: 1) Unterstützung der Diagnostik \& Therapie der Epilepsie am Universitätsklinikum in Tübingen, 2) Unterstützung des Tumorboards bei Patienten mit Kolonkarzinom und Ko-Morbidiäten am Johanniter-Krankenhaus in Bonn, 3) Unterstützung der Langzeitnachsorge nach Stammzelltransplantation am Robert-Bosch-Krankenhaus Stuttgart. Die Evaluationsergebnisse werden im Februar 2019 vorliegen. 


\section{PS003}

\section{Entwicklung und Evaluation eines Expertensystems zur Diagnosefindung im Rahmen der Weiterbildung in der Inneren Medizin (http://www.diagnosemanager.de)}

\section{Wolfram Arends}

diagnosemanager.de, Allgemeinmedizin, Tutzing, Germany

Die rasche Interpretation der klinischen Befunde und die damit verbundene Einordnung in das entsprechende Illness Script ist eine geforderte Tätigkeit im Rahmen der Weiterbildung. Aufgrund des Wissenszuwachs in der Medizin in Form von neuen Befundmustern, Leitsymptomen und klinischen Bildern wird in der allgemeinmedizinischen Praxis eine Expertise benötigt, welche von jungen Kollegen in der Weiterbildung zum Teil erst kontinuierlich erarbeitet werden muß.

Es wurde ein internetbasiertes Programm zur Diagnosefindung erstellt. Berücksichtigt wurden dabei Beratungsanlässe der hausärztlichen Tätigkeit zu einzelnen medizinischen Krankheitsbildern. Das Programm überprüft die angewählten Symptome zur Erarbeitung der möglichen Arbeitsdiagnose. Beim Ablauf erfolgt zunächst die Befundeingabe durch das Anwählen von insg. 2.375 Symptomen. Im Rahmen der Auswertung greift das Programm auf 2.484 klinische Befundmuster zurück. Zusätzlich können noch 30 Befundscores zur Einteilung abgerufen werden. Das Programm wird über folgenden Link aufgerufen: http://www.diagnosemanager.de

Programmiert wurde mit den Sprachen html und php4 über einen Apache Server.

Die Evaluation erfolgte anhand eines Fragebogens, welcher $u$. a. Akzeptanz, Lehrqualität und Motivation beinhaltet. Die Prozessevaluation dieser Anwendung ergibt eine breite Akzeptanz sowie eine gute messbare Wirkung hinsichtlich der extrinsischen und intrinsischen Motivation.

Das Programm stellt ein mögliches Werkzeug zum Erlernen der Hypothesenbildung im Rahmen der Inneren Medizin dar.

Custers E.J.,Boshuizen, $\mathrm{H}$, Schmidt $\mathrm{H}$, The Role of Illness Scripts in the Development of Medical Diag. Expertise: Results From an Interview Study. Pages 367-398 | Published online: 14 Dec 2009

Custers E.J. et. al Thirty years of illness scripts: Theoretical origins and practical applications. Med Teach. 2015, May;37(5):457-62. doi: 10.3109/0142159X.2014.956052. Epub 2014 Sep 2

Arends, W., Eitel, F. (1998): Programmierung und Evaluation eines interaktiven computerunterstützen Selbstlernprogramms „Rundherd in der Lunge" in Computer based Training in der Medizin, GMDS München 1998 Shaker Verlag S.117-123 "Methoden, Techniken, Anwendungen, GMDS,

\section{PS004}

Telemedizinische Betreuung von Patienten mit chronischer Herzinsuffizienz: eine gesundheitsökonomische Evaluation aus der Perspektive der Versorgungsrealität

Sebastian Liersch'; Wolfgang-Michael Franz ${ }^{2}$; Stefanie Kornek'; Jürgen Effenberger ; ${ }^{1}$ Stephanie Sehlen'; Markus Ziegler ${ }^{3}$; Werner Wyrwich ${ }^{1}$

${ }^{1} \mathrm{AOK}$ Nordost, Versorgungsmanagement, Berlin, Germany; ${ }^{2} \mathrm{SHL}$ Telemedizin $\mathrm{GmbH}$, Medizinischer Direktor, München, Germany; ${ }^{3}$ Medizinische Informatik, -, Berlin, Germany

Einleitung: Das telemedizinische Betreuungsprogramm „AOK-Curaplan Herz Plus" der AOK Nordost ergänzt in Berlin und Brandenburg die Routineversorgung von Patienten mit chronischer Herzinsuffizienz (CHI). Dabei werden täglich Symptome der CHI wie Gewicht telemetrisch übertragen um eine drohende Dekompensation rechtzeitig zu erkennen. Bei einer Verschlechterung des Gesundheitszustandes werden den Patienten konkrete Handlungsempfehlungen gegeben oder der Besuch des behandelnden Arztes angeraten. Zur Stärkung des Selbstmanagements erhalten Patienten regelmäßig individuelle, telefonische Schulungs- und Beratungsgespräche durch kardiologisch qualifiziertes Fachpersonal. Seit 2006 haben an diesem Programm rund 16.500 Patienten teilgenommen. Die Evaluation gibt Aufschluss darüber, inwieweit sich die langjährige telemedizinische Betreuung auf Mortalität sowie Leistungsinanspruchnahme in der Versorgungsrealität auswirkt.

Methoden: Zielgruppe sind Patienten mit einer stationären Hauptoder Nebendiagnose, die mit CHI assoziiert ist. In der Evaluation werden unter 18-Jährige, stationär Pflegebedürftige, Patienten mit anderen schweren Erkrankungen, wie z. B. manifesten psychischen Erkrankungen, dialysepflichtiger Niereninsuffizienz oder Listung zur Herztransplantation ausgeschlossen. Im Intention-to-treat-Ansatz werden alle Patienten, die sich zwischen Januar 2014 und Juni 2017 in das Programm eingeschrieben haben berücksichtigt und für mindestens ein Jahr nachbeobachtet. Eine vergleichbare Kontrollgruppe wurde mittels Propensity Score-Matching aus den Routinedaten der AOK Nordost gebildet. Primäres Outcome ist das Überleben ein Jahr nach Programmaufnahme. Die Leistungsinanspruchnahme wird über Anzahl und Verweildauer der Krankenhausaufenthalte dargestellt.

Ergebnisse: Insgesamt haben sich im Beobachtungszeitraum vom 01.01.2014-30.06.2018 $n=9.472$ Patienten in das Betreuungsprogramm eingeschrieben, welche im Mittel 1,9 Jahre teilnahmen. Durchschnittlich lag das Alter der Patienten bei 73 Jahren, wobei Frauen (46\%) im Mittel 3,6 Jahre älter sind $(p<0,001)$. Die Patienten wiesen zu Teilnahmebeginn folgende NYHA-Stadien auf: I (5\%), II (30\%), III (31\%), IV (13\%). Bei $11 \%$ wurde das Stadium nicht näher bezeichnet. Insgesamt versterben $6 \%$ der Patienten innerhalb von 12 Monaten nach Teilnahmebeginn; die 24-Monats-Mortalität beträgt $9 \%$. In den ersten 12 Monaten waren die Patienten aufgrund einer Kreislauferkrankung durchschnittlich 1,3-mal im Krankenhaus. Bezogen auf die ersten 24 Monate steigt die Rate auf 2,3 an.

Diskussion: Die Evaluation des langjährigen telemedizinischen Betreuungsprogramms umfasst erstmalig einen Beobachtungszeitraum bis zu 4,5 Jahren sowie eine vergleichsweise sehr hohe Teilnehmerzahl. Sie basiert auf Real World Data, die nicht primär zur Bewertung von medizinischen Interventionen erhoben wurden. Die Sekundärdatenanalyse erfolgt aus der Perspektive der Versorgungsrealität. Die endgültigen Ergebnisse werden auf dem Kongress präsentiert. 


\section{PS005}

Interference of tumor mutational burden with outcome of patients with head and neck cancer treated with definitive chemoradiation: A multicenter retrospective study of the German Cancer Consortium Radiation Oncology Group (DKTK-ROG)

Theresa Eder'; AK Hess ${ }^{2}$; R Konschak ${ }^{3}$; C Stromberger ${ }^{2}$; K Jöhrens ${ }^{4}$; V Fleischer $^{2}$; M Hummel $^{12}$; P Balermpas ${ }^{5}$; J von der Grün ${ }^{5}$; A Linge ${ }^{6}$; FA Lohaus ${ }^{6}$; M Krause $^{6}$; M Baumann ${ }^{6}$; M Stuschke ${ }^{7}$ D Zips ${ }^{8}$; AL Grosu'; A Abdollahi ${ }^{10}$; J Debus $^{10} ;$ C Belka $^{11} ;$ S Pigorsch $^{11} ;$ SE Combs ${ }^{11} ;$ V Budach $^{3}$; I Tinhofer $^{3}$

${ }^{1}$ Charité - Universitätsmedizin Berlin, Labor für Strahlenbiologie, Berlin, Germany;

${ }^{2}$ Charité University Hospital, Berlin, Germany, Department of Radiooncology and Radiotherapy, Berlin, Germany;

${ }^{3}$ German Cancer Research Center (DKFZ), Heidelberg and German Cancer Consortium (DKTK) partner site Berlin, Department of Radiooncology and Radiotherapy, Charité University Hospital, Berlin, Germany, Berlin, Germany;

${ }^{4}$ Charité University Hospital Berlin, Berlin, Germany, Institute of Pathology, Berlin, Germany;

${ }^{12}$ German Cancer Research Center (DKFZ), Heidelberg and German Cancer Consortium (DKTK) partner site Berlin, Institute of Pathology, Charité University Hospital Berlin, Berlin, Germany, Berlin, Germany;

${ }^{5}$ German Cancer Research Center (DKFZ), Heidelberg and German Cancer Consortium (DKTK) partner site Frankfurt, Department of Radiotherapy and Oncology, Goethe-University Frankfurt, Germany, Frankfurt, Germany;

${ }^{6}$ German Cancer Research Center (DKFZ), Heidelberg and German Cancer Consortium (DKTK) partner site Dresden, OncoRay - National Center for Radiation Research in Oncology, Faculty of Medicine and University Hospital Carl Gustav Carus, Technische Universität Dresden, Helmholtz-Zentrum Dresden - Rossendorf, Dresden, Germany, Department of Radiotherapy and Radiation, Dresden, Germany;

${ }^{7}$ German Cancer Research Center (DKFZ), Heidelberg and German Cancer Consortium (DKTK) partner site Essen, Department of Radiotherapy, Medical Faculty, University of Duisburg-Essen, Essen, Germany, Essen, Germany;

${ }^{8}$ German Cancer Research Center (DKFZ), Heidelberg and German Cancer Consortium (DKTK) partner site Tübingen, Department of Radiation Oncology, Faculty of Medicine and University Hospital Tübingen, Eberhard Karls Universität Tübingen, Germany, Tübingen, Germany;

${ }^{9}$ German Cancer Research Center (DKFZ), Heidelberg and German Cancer Consortium (DKTK) partner site Freiburg, Department of Radiation Oncology, University of Freiburg, Germany, Freiburg, Germany;

${ }^{10}$ German Cancer Research Center (DKFZ), Heidelberg and German Cancer Consortium (DKTK) partner site Heidelberg, Heidelberg Institute of Radiation Oncology (HIRO), National Center for Radiation Research in Oncology (NCRO), University of Heidelberg Medical School and German Cancer Research Center (DKFZ), Germany, Heidelberg, Germany;

${ }^{11 G e r m a n}$ Cancer Research Center (DKFZ), Heidelberg and German Cancer Consortium (DKTK) partner site Munich, Department of Radiotherapy and Radiation Oncology, Ludwig-Maximilians-Universität, Munich, Germany, Munich, Germany;

Background: Tumor mutational burden (TMB) estimated from whole exome sequencing (WES) or comprehensive gene panels has previously been established as predictive factor of response to immune checkpoint inhibitors (ICI). Its predictive value for the efficacy of concurrent chemoradiation (cCRTX), a potential combination partner of $\mathrm{ICl}$, remains unknown.
Methods: The accuracy of TMB estimation by an in-house 327-gene panel was established in the TCGA HNSCC dataset. Interference of TMB with outcome after CCRTX was determined in a multicenter cohort of patients with locally advanced head and neck squamous cell carcinoma (HNSCC) uniformly treated with CCRTX. tNGS was successfully applied in 101 formalin-fixed, paraffin-embedded pretreatment tumor samples. In a subset of cases $(n=40)$, tumor RNA was used for immune-related gene expression profiling by the nanoString platform. TMB was correlated with TP53 genotype, HPV status, immune expression signatures and survival parameters. Results were validated in the TCGA HNSCC cohort.

Results: A high accuracy of TMB estimation by the 327-gene panel was established. High TMB was significantly associated with an increased prevalence of TP53 mutations and immune gene expression patterns unrelated to $T$ cell-inflamed gene expression profiles. Kaplan-Meier analysis revealed significantly reduced overall survival in the patient group with high TMB (hazard ratio for death: 1.79, 95\% confidence interval: 1.02-3.14; $\mathrm{P}=0.042$ ) which remained significant after correcting for confounding factors in the multivariate model. The prognostic value of TMB was confirmed in the TCGA HNSCC cohort.

Conclusion: High TMB identifies HNSCC patients with poor outcome after CCRTX who might preferentially benefit from CRTX-ICl combinations.

\section{PS006 \\ Design eines virtuellen Therapieunterstützungssystems für Herzinsuffizienz-Patienten - Vorstellungen und Anforderungen der Patienten}

Alexander Palant'; Bettina Zippel-Schultz'; Thom Hoedemakers²; Olga Golubnitschaja ${ }^{3}$; Hans-Peter Brunner - La Rocca ${ }^{4}$; Thomas M. Helms ${ }^{1}$

${ }^{1}$ Deutsche Stiftung für chronisch Kranke, -, Berlin, Germany; ${ }^{2}$ Sananet Care BV, -, Sittard, Netherlands; ${ }^{3}$ Universitätsklinik Bonn, -, Bonn, Germany; ${ }^{4}$ Maastricht UMC+, Kardiologie, Maastricht, Netherlands

Bereits jetzt ist die Herzinsuffizienz (HI) eine der häufigsten chronischen Erkrankungen der inneren Medizin und ist durch eine hohe Morbidität und Mortalität gekennzeichnet [1]. In den westlichen Ländern sind etwa $2 \%$ der Bevölkerung von einer HI betroffen - Tendenz steigend [2]. Aufgrund des wachsenden Ungleichgewichts zwischen Behandlungsbedarf und Versorgungsangebot stellt die $\mathrm{HI}$ eine der großen Herausforderungen des Gesundheitssystems dar [3].

Um in Zukunft eine optimale Versorgung der HI gewährleisten zu können, bedarf es alternativer Lösungen. Weitgehendes Selbstmanagement mit Hilfe von Anwendungen der Digitalen Medizin könnte ein Weg sein, diese Herausforderungen zu bewältigen [3]. Darüber hinaus schafft der Einsatz von künstlicher Intelligenz neue Möglichkeiten, die gesundheitliche Versorgung zu verbessern. Diese Maßnahmen können zu massiven Kosteneinsparungen im Gesundheitswesen führen.

Langfristiges Ziel des Projektes PASSION-HF (gefördert durch Interreg NWE, 2018-2021) ist die Entwicklung einer virtuellen Ärztin "Abby“, die Patienten mit chronischer $\mathrm{HI}$ im Alltag begleitet. Die Akzeptanz und Nutzung einer solchen Lösung hängt zu einem großen Teil von der Einstellung der Patienten gegenüber einem virtuellen Therapieunterstützungssystem und dem von ihnen erwarteten Nutzen ab. In dem Projekt werden daher im ersten Schritt die Anforderungen und Bedürfnisse der Patienten an ein solches System erhoben. Die Studie im Mixed-Methods-Design wird an vier Zentren aus Deutschland, den Niederlanden, Großbritannien und Irland durchgeführt. Semi-strukturierte Interviews werden durch einen standardisierten Fragebogen ergänzt. Befragt werden jeweils Patienten und deren, in 
die Versorgung eingebundenen, Angehörige. Folgende Fragen stehen im Fokus: Welchen Unterstützungsbedarf sehen Patienten mit $\mathrm{HI}$ (a) im alltäglichen Leben und (b) im aktuellen Versorgungsprozess der Erkrankung? Wie ist die generelle Einstellung der Patienten gegenüber digitalen Technologien? Welche Unterstützung wünschen sich die Patienten durch ein virtuelles Therapieunterstützungssystem? Welche Anforderungen muss ein solches System erfüllen? Was motiviert Patienten (a) sich therapieadhärent zu verhalten, (b) die digitale Lösung zu nutzen? Welche Unterschiede bestehen zwischen den Ländern bezüglich der Ergebnisse? Die Interviews werden mittels Inhaltsanalyse nach Mayring, die Fragebögen mittels deskriptiver Statistik ausgewertet.

Die im Frühjahr des nächsten Jahres vorliegenden ersten Ergebnisse der Studie geben einen Einblick in die Bedürfnisse, Anforderungen und Wünsche der Patienten und deren Angehörigen zu Design und Funktionen eines digitalen Therapieunterstützungssystems. Sie sind ein wichtiger Baustein im weiteren Entwicklungsprozess von PASSION-HF und werden erstmalig auf dem Kongress der DGIM vorgestellt.

[1] McMurray JJV, S.S., The burden of heart failure. European Heart Journal Supplements, 2002. 4: p. D50-D58.

[2] Heider, D., et al., Health care costs in the elderly in Germany: an analysis applying Andersen's behavioral model of health care utilization. BMC Health Serv Res, 2014. 14: p. 71.

[3] Brunner-La Rocca, HP., et al. Challenges in personalised management of chronic diseases - heart failure as prominent example to advance the care process. EPMA J, 2016. 7: p. 2

\section{PS007 \\ Weiterbildung zum eHealth-Berater - Evaluationsergebnisse der Pilotphase}

Christoph Kokelmann

Tumaini Institut für Präventionsmanagement $\mathrm{GmbH}$, eHealth, Dresden, Germany

Fragestellung: Die Digitalisierung der Lebensbereiche verändert auch den Beratungsalltag in Therapie und Prävention. Unter dem Begriff eHealth entfaltet sich eine Vielzahl an Gesundheits-Apps, medi-zinischen Wearables und Internetportalen. Wie müssen Akteure des Gesundheitswesens daher weitergebildet werden, um diesen $\mathrm{He}$ rausforderungen adäquat begegnen zu können?

Methodik: Das TUMAINI-Institut entwickelt eine eHealth-Weiterbildung und ein Online-Netzwerk für Ak-teure im Gesundheitswesen. Zur Zielgruppe gehören vorrangig Gesundheitsakteure mit Patien-tenkontakt. Zur Bedarfserhebung wurde eine Online-Befragung $(n=85)$ durchgeführt. Bereits bestehende curriculare Ansätze sowie wissenschaftliche Online-Netzwerke wurden systema-tisch recherchiert und ausgewertet. Die Pilotkurse der Weiterbildung wurden durch das Institut für Klinische Psychologie und Psychotherapie der TU Dresden mittels qualitativer und quantita-tiver Befragung der Teilnehmer evaluiert. Zudem wurden die Teilnehmer der Pilotkurse aktiv in die Ausgestaltung des Online-Netzwerkes eingebunden.

Ergebnisse: Die Erhebung zum Fortbildungsbedarf identifizierte Defizite der Zielgruppe im Umgang mit und in der Bewertung von eHealth-Anwendungen. Die Evaluation der beiden Pilotkurse zeigt einen deutlichen Wissenszuwachs der Teilnehmer $(n=20)$ sowie eine positive Einschätzung der Kurs-inhalte hinsichtlich der Relevanz für den Arbeitsalltag. Parallel wird das Online-Netzwerk (eHealthWerk. de) bis Frühjahr 2019 vollumfänglich verfügbar sein und eine aktive
eHealth-Community fördern sowie einen E-Learning-Bereich, aus der Weiterbildung bekannte, aktuell gehaltene Infomaterialen und aktuelle Blog-Beiträge bieten.

Schlussfolgerung: Die Ergebnisse der Evaluation bestätigen, dass es sich um einen vielversprechenden Ansatz in der Prävention lebensstilassoziierter Erkrankungen wie Diabetes mellitus handelt. In Zusam-menhang mit den umfangreichen Fortbildungsunterlagen und der parallelen Vernetzung der Teilnehmer im Online-Netzwerk „eHealthWerk" wird die - vorwiegend digitale - Gesundheits-kompetenz der Teilnehmer nachhaltig gefördert. Die zukünftigen eHealth-Berater haben damit das Potential, eHealth-Anwendungen als zielgerichtete und individuelle Lösung zur Unterstüt-zung des Selbstmanagements der Patienten einzusetzen.

\section{PS008 \\ Evaluation of costs, utilization and mortality of a project for telemonitoring of patients with chronic heart failure}

Stefan Rabbe'; Jonas Schreyögg ; Rudolf Blankart ${ }^{2}$; Lutz Hager; ${ }^{3}$ Martin Lehner ${ }^{4}$; Wolfgang-Michael Franz ${ }^{5}$

'Universität Hamburg, Hamburg Center for Health Economics, Hamburg, Germany; ${ }^{2}$ Universität Bern, Kompetenzzentrum für Public Management, Bern, Switzerland; ${ }^{3}$ IKK Südwest, Geschäftsführer, Saarbrücken, Germany; ${ }^{4}$ SHL Telemedizin GmbH, CEO SHL-Telemedizin GmbH, München, Germany; ${ }^{5} \mathrm{SHL}$ Telemedizin $\mathrm{GmbH}$, Medizinischer Direktor SHL-Telemendizin $\mathrm{GmbH}$, München, Germany

Background: The program "ZERTIVA" operated by SHL-Telmedizin aims at optimizing the therapy for high risk patients of the sickness fund IKK Südwest with chronic heart failure especially within the New York Heart Association (NYHA) classes II-IV. The patients were equipped with telemedical devices such as a body-scale, a blood pressure monitor or a 12 channel ECG system. With these devices information are collected in electronic medical records and analysed by a medical professional who contacts the patient after abnormal changes in the measurements aiming on early interventions to administer the episode. The $24 / 7$ intervention program is supported by coaching modules related to co-morbidities. All patient related data were provided by IKK Südwest.

Methodology: For the evaluation the patient collective of the German sickness fund IKK Südwest was screened for eligibility for the program. Inclusion criteria are at least one inpatient stay due to heart failure or related conditions within the past 24 months before the start of the program as well as at least one outpatient diagnosis of heart failure or heart failure related diseases. Exclusion criteria are defined to eliminate patients with diagnoses such as cancer treatments, dialysis or mental illnesses.

After the first selection of all relevant patients for the study population patients are randomly assigned to either intervention or control group. To ensure pre-treatment balance on important characteristics between treatment- and control group different methods such as propensity score matching and propensity weighting are used. For these methods patient characteristics e.g., on costs, medications and comorbidities are used for the period of 24 months before the start of the intervention. Intervention groups were enrolled in 11 waves. The observation period was 24 months (Q3/2015-Q2/2017).

In a first analysis total health care costs consisting of inpatient and outpatient care, pharmaceutical as well as medical aids were analysed.

Following this outpatient contacts, length of hospital stays and the number of prescribed drugs related to heart failure will be analysed. 
Further, mortality will be used as an outcome parameter.

Results: Preliminary results for the Gamma regression after propensity score matching on 714 patients in each group suggest a significant negative effect of the intervention of $0,1431(p=0,005)$ for the total cost in the study, leading to an average decrease in cost of $-2.187 €$ per patient. There were 998 hospitalizations accounted for in the intervention group vs. 1197 hospitalizations in the control group.

These effects appear to be driven by a decrease in inpatient costs, while costs in other areas such as outpatient care or drugs increased slightly.

Conclusion/Outlook: The preliminary results using propensity score matching suggest a sizable cost reduction for patients in the telemedical treatment group. Results on utilization and outcomes are still pending.

\section{PS009 \\ PICASO - Die Plattform für eine verbesserte persönliche, koordinierte Betreuung chronisch Kranker}

Jutta G. Richter'; Gamal Chehab'; Monika Tomczak'; Hasan Acar'; Henrike Gappa ${ }^{2}$; Elisabeth Ricken'; Catarina Schwartz'; Matthias Schneider'; Carlos Velasco ${ }^{2}$; Konsortium PICASO ${ }^{3}$

${ }^{1}$ Medzinische Fakultät, Universitätsklinikum Düsseldorf, Heinrich-Heine-Universität Düsseldorf, Poliklinik, Funktionsbereich \& Hiller Forschungszentrum für Rheumatologie, Düsseldorf, Germany; ${ }^{2}$ Fraunhofer-Institut für Angewandte Informationstechnik FIT, Fraunhofer-Institut für Angewandte Informationstechnik FIT, Sankt Augustin, Germany; ${ }^{3}$ PICASO Konsortium, PICASO Konsortium, http://www.picaso-project.eu, Germany

Hintergrund: Die sektorübergreifende Koordination von Behandlungsplänen und ein effizientes Management von Patienten (Pat.) mit Komorbiditäten sind von großer Bedeutung. Dies betrifft auch Pat. mit rheumatoider Arthritis (RA). Eine Orchestrierung der an verschiedenen Stellen über einen Pat. verfügbaren Informationen ist von großer Bedeutung, um eine effektive Datennutzung, sinnvolle Managementprozesse, Vermeidung redundanter Diagnostik und folglich Kosteneffizienz zu gewährleisten. Die im Rahmen des Horizon2020-geförderten PICASO-Projekts (www.picaso-project.eu) entwickelte Informations- und Kommunikationsplattform unterstützt die Orchestrierung von Pat.-Daten entlang des Versorgungskontinuums bestehend aus Krankenhäusern, Ambulanzen, Praxen, nicht-ärztlichen Gesundheitsdienstleistern bis zum häuslichen Pat.-Monitoring. Methodik: PICASO verfolgt einen nutzerzentrierten Ansatz, d.h. durch Workshops und Interviews mit Ärzten verschiedener Disziplinen, Pat. und weiteren Stakeholdern des Gesundheitswesens (z.B. Datenschutzbeauftragte) wurden Anforderungen an die Plattform ermittelt. Ethische Fragestellungen bearbeitet ein Begleitgremium, dem auch externe Mitglieder und Pat. angehören. Ein umfangreiches Framework dient der Evaluation (u.a. zu Nutzerzufriedenheit, klinischer Relevanz der Entwicklungen) der 6-monatigen Proof-of-concept Studie mit RA-Pat. und Ärzten. Ein positives Ethikvotum der Ethikkommission der Medizinischen Fakultät der Heinrich-Heine-Universität Düsseldorf liegt vor. Ergebnisse: Zur Entwicklung der Plattform wurden 111 identifizierte Nutzeranforderungen herangezogen. Zudem wurde auf Konformität mit der europäischen Datenschutzgrundverordnung sowie nationalen Regelungen in Deutschland geachtet. Um den Datenaustausch mit anderen Softwaresystemen im Gesundheitswesen zu ermöglichen, basieren alle Entwicklungen auf dem neuen HL7 Standard ,Fast Healthcare Interoperability Resources' (FHIR). Dieser bietet viele Vorteile wie ein semantisches Modell zur Beschreibung kleinster Einheiten (z.B. Medikationseinnahmezeitpunkte, diagnostische Verfahren), sodass Informationen hierüber miteinander verknüpft und bereitgestellt werden können. Dabei verbleiben die Daten beim Dateneigner. Rollenspezifische Datenzugriffe sind möglich. Bislang wurden 27 RA-Pat. (81,5\% weiblich) rekrutiert, das mittlere Alter betrug 59,2 $\pm 10,9$ Jahre, die Krankheitsdauer $13,2 \pm 8,7$ Jahre. Neben dem positiven Feedback bei Rekrutierung können während des DGIM-Kongresses Ergebnisse der begonnenen Nutzerevaluationen präsentiert werden. Zusammenfassung: Die PICASO-Plattform bietet eine sektorübergreifende Orchestrierung von Pat.-Daten und damit moderne Möglichkeiten für sinnvolle Managementprozesse (z.B. Treat-to-Target, Telemonitoring). Sie soll für RA-Pat. aber auch für eine Anwendbarkeit bei anderen chronischen Krankheiten verfügbar sein und hat das Potenzial, das Gesundheitssystem zu verändern.

Acknowledgement: This project received funding from the European Union's Horizon 2020 research and innovation programme under grant agreement No 689209

\section{Innere Medizin integral}

\section{PS010 \\ Einfluss neuer HCC-Therapeutika auf die Regulation der p53-Familie und die Induktion von Zelltod in Hepatomzelllinien}

Patricia-luliana Mester; Elisabeth Aschenbrenner; Kirstin Pollinger; Heidi Gschwendter; Karsten Gülow; Claudia Kunst; Martina Müller-Schilling

Universitätsklinikum Regensburg, Innere Medizin I, Regensburg, Germany

Hintergrund: Das hepatozelluläre Karzinom (HCC) ist die dritthäufigste Ursache für tumorbedingte Todesfälle weltweit. Das HCC weist zudem eine hohe Resistenz gegenüber Chemotherapeutika auf. Die HCC-Behandlung kann in chirurgische und nicht-chirurgische Therapien unterteilt werden, die leberspezifisch oder systemisch sind. Bis 2018 war Sorafenib die einzige orale systemische Therapie für Patienten mit nicht resektablem HCC. Jedoch konnte Sorafenib ausschließlich bei Patienten mit guter Leberfunktion (Child-Pugh A), einem HCC Stadium BCLC-C oder Tumoren in einem früheren Stadium angewendet werden, die schnell wachsend sind oder bei denen keine lokale ablative oder chirurgische Therapie möglich war. Mit den „EASL Clinical Practice Guidelines: Management of hepatocellular carcinoma" wurden neue Substanzen empfohlen und zugelassen, die das therapeutische Repertoire für HCC erweitern. Der Tumorsupressor p53 ist nicht nur das häufigste mutierte Gen bei Tumoren, sondern auch zusammen mit p63 und p73 - ein essentieller Regulator des Zelltods. Da die molekularen Wirkungsmechanismen der neuen HCC-Therapeutika nur teilweise verstanden sind, war das Ziel dieser Studie die Identifikation der Mechanismen, über die diese Substanzen Zelltod im HCC induzieren.

Methoden: HepG2 humane Zellen wurden mit den Serumkonzentrationen der HCC-relevanten Therapeutika (Regorafenib $5 \mu \mathrm{M}$, Sorafenib $4.5 \mu \mathrm{M})$ für 24 - 72 h inkubiert. Cabozantinib $(2 \mu \mathrm{M})$, Lenvatinib $(2,34 \mu \mathrm{M})$, Ramucirumab $(6,96 \mu \mathrm{M})$ und Bleomycin $(4 \mu \mathrm{M})$ wurden in $2 \mathrm{x}$ Serumkonzentration eingesetzt. DMSO wurde als Kontrolle benutzt. Pro- und antiapoptotische Mitglieder der $\mathrm{Bcl}-2$ Familie wurden auf mRNA-Ebene mittels qPCR untersucht. Die Untersuchung der Proteine der p53-Familie erfolgte mittels Western Blot. Die Zellviabilität wurde mittels MTS-Assay analysiert und der Zelltod mittels Durchflusszytometrie nach DAPI/Annexin V Färbung bestimmt.

Ergebnisse: Die Viabilität der HepG2 Zellen war $48 \mathrm{~h}$ nach Behandlung mit Bleomycin, Sorafenib, Regorafenib und Cabozantinib um nahezu 50\% reduziert. Die Ergebnisse der Durchflusszytometrie zeigten, dass Sorafenib und Regorafenib zwar Zelltod, aber keine Apoptose in 
Hep G2 Zellen induzieren. Nach Behandlung mit Sorafenib, Regorafenib und Cabozantinib zeigte sich eine Induktion von p63 und p73 und nur partiell eine Induktion von p53. Eine Spaltung von Caspase-3 sowie die Induktion proaptotischer Mitglieder der Bcl-2 Familie (Bax, Bak) konnten durch diese Targeted Therapies nicht induziert werden.

Schlussfolgerung: Neue HCC-Therapeutika können Zelltod in Hepatomzellen induzieren. Jedoch, auch wenn p53 von Sorafenib, Regorafenib und Cabozantinib hochreguliert wird, findet der Zelltod über einen apoptose-unabhängigen Mechanismus statt. Diese Ergebnisse erweitern nicht nur unsere Kenntnisse bezüglich der Wirkungsweise der HCC-Therapeutika, sondern deuten darüber hinaus darauf hin, dass die p53-Familie auch an der Regulation anderer apoptose-unabhängiger Zelltodarten beteiligt ist.

\section{PS011}

\section{Ultraschall gesteuerte Biopsie bei onkologischen Patienten}

Thorsten Nitsch; Thomas Südhoff

Klinikum Passau, II. Med, Passau, Germany

Einführung: Die Histologie-Gewinnung ist die Grundlage für die Therapieplanung in der Hämatologie und Onkologie. Es gibt verschiedene Wege eine verlässliche Histologie zu erhalten, wie z.B. Endoskopie, CT gesteuerte Biopsie oder es ist in manchen Fällen sogar ein chirurgischer Eingriff notwendig.

Die Sonographie ist eine einfache und häufig verfügbare Methode mit der auch eine Ultraschall-gesteuerte Biopsie erfolgen kann. Wir berichten hier über die Zielregionen, Effektivität und die Komplikationsrate dieser Methode.

Methode: Dieses ist eine retrospektive Analyse der Ultraschall-gesteuerten Biopsien in unserer Abteilung. Die Biopsien wurden mit einem Toshiba 400 Ultraschallgerät durchgeführt. Je nach Lokalisation erfolgte die Punktion mit einem "convex array“ Punktionsschallkopf oder mit einem Linearschallkopf in "Freihand"-Technik. Wir benutzten True-cut Nadeln (Achieve Programmable Automatic Biopsy systems) mit der Größe von 14G bis 18G, insbesondere $16 \mathrm{G}$. Die Biopsien wurden unter Lokalanästhesie und unter aseptischen Bedingungen durchgeführt. Bei jeder Lokalisation erfolgten durchschnittlich drei Biopsien und eine Zytologie-Gewinnung. Komplikationen wurden in drei Grade eingeteilt: 1 für geringe bis zu 3 für schwere Komplikationen.

Ergebnis: Wir haben 1153 Biopsien mit folgenden Lokalisationen durchgeführt: Lymphknoten: 379, Leber: 316, Lunge: 125, Weichteile (z.B. Muskel, Haut): 110, Osteolysen: 54, Pankreas: 34, Abszess: 32 Peritoneum: 28, Brustdrüse: 23 , Pleura: 10 , Mediastinum: 8 , Niere 8 , Schilddrüse: 6, Lymphozele: 5, Nebenniere: 5, Retroperitoneum: 5, Milz: 2, Dünndarm: 1, Parotis 1, Zyste: 1.

In 96\% der Fälle erhielten wir eine verlässliche Histologie, die keine weitere Intervention erforderte.

Komplikationen traten insgesamt in 4,5\% der Fälle auf. Am häufigsten $(3,7 \%)$ handelte es sich um geringe Komplikationen, wie ein kurzer selbstlimitierter Schmerz oder geringe selbstlimitierte Blutungen. Moderate Komplikationen waren mit 0,5\% zu verzeichnen (wie z.B. ein Pneumothorax). Schwere Komplikationen traten bei 0,3\% der Fälle auf, hierbei handelte es sich um Blutungen mit folgenden Interventionen. Todesfälle waren nicht zu verzeichnen.

Zusammenfassung: Unsere Analyse zeigt, dass die Ultraschall-gesteuerte Biopsie bei einem breiten Spektrum an Lokalisationen sicher und effektiv durchgeführt werden kann.
Eine verlässliche Histologie konnten mit einer hohen Rate von 96\% erzielt werden, so dass keine weitere Intervention notwendig war. Zusätzlich ist es eine sichere Methode mit einer niedrigen Komplikationsrate von insgesamt 4,5\% hier insbesondere leichte Komplikationen (3,7\%). Hieraus schließen wir, dass die Ultraschall-gesteuerte Biopsie ein effektives Werkzeug ist, welches das Potential hat, das diagnostische Vorgehen zu verkürzen.

\section{PS012 \\ Characterization of the (epi)genetic alterations during liver cancer development and progression.}

Carolin Czauderna ${ }^{10}$; A. Poplawski'; C. Castven ${ }^{2}$; S. Heilmann-Heimbach ${ }^{3}$; M. Odenthal $^{4}$; W. Amer ${ }^{4}$; J. B. Andersen ${ }^{5}$; C. O'Rourke ${ }^{5}$; H. Binder ${ }^{8}$; D. Ridder ${ }^{2}$; B. Straub $^{2}$; P. R. Galle ${ }^{9}$; S.S. Thorgeirsson ${ }^{6}$; Y. N. Park'; J. U. Marquardt ${ }^{2}$

'Klinikum der Johannes Gutenberg-Universität Mainz, Institute of Medical Biostatistics, Epidemiology and Informatics (IMBEI) Division Biostatistics and Bioinformatics, Mainz, Germany; ${ }^{2}$ Universitätsklinikum Mainz, Department of Medicine I, Mainz, Germany; ${ }^{3}$ Universitätklinikum Bonn, Institute of Human Genetics, Department of Genomics, Life \& Brain Center, Bonn, Germany: ${ }^{4}$ Klinikum der Universität zu Köln, Insitute of Pathology, Köln, Germany; ${ }^{5}$ University of Copenhagen, Biotech Research and Innovation Centre (BRIC), Dept. of Health and Medical Sciences, Kopenhagen, Denmark; ${ }^{6}$ National Cancer Institute, NIH, Laboratory of Experimental Carcinogenesis (LEC), Center for Cancer Research, Washington DC, United States; ${ }^{7}$ Yonsei University, ${ }^{7}$ Department of Pathology, Brain Korea ${ }^{21}$ Project for Medical Science, Integrated Genomic Research Center for Metabolic Regulation, Seoul, Republic of Korea; ${ }^{8}$ Universitätsklinikum Mainz, Institute of Medical Biostatistics, Epidemiology and Informatics (IMBEI) Division Biostatistics and Bioinformatics, Mainz, Germany; ' 'Universitätsklinikum Mainz, Department of Pathology, Mainz, Germany; ${ }^{10}$ Johannes-Gutenberg-Universitätsmedizin Mainz I. Medizinische Klinik und Poliklinik, Mainz, Germany

Background and Aims: Development of primary liver cancer is a multi-stage process. Pre-neoplastic dysplastic lesions emerge on the basis of chronic liver damage and evolve into early hepatocellular carcinoma (eHCC) and, subsequently, progressed HCC (pHCC). Detailed molecular characterization and prediction of pre-neoplastic lesions at high risk for malignant transformation would significantly advance our diagnostic and therapeutic approaches. We here utilized integrative molecular analyses to characterize the sequential evolution of liver cancer and aimed to define key epigenetic drivers and biomarkers of HCC development and progression.

Methods: Methylation 450k-beadchip analyses were performed on cirrhotic liver $(n=7)$, low- $(n=4)$ and high-grade $(n=9)$ dysplastic lesions, eHCC $(n=5)$ and $\mathrm{pHCC}(n=3)$ from 8 HCC patients with chronic hepatitis $B$ infection. Differentially methylated gene regions (DMGR) were identified in comparison to non-cirrhotic and non-infected liver $(n=9)$. Potential epi-drivers and biomarkers were identified by integrative analyses of transcriptomic changes and validated in an independent cohort from the TCGA database.

Results: The proportion of hypermethylated DMGR progressively increased from cirrhosis over dysplastic- to HCC and peaked in eHCC lesions. Early epigenetic alterations involved signaling pathways related to cell death, apoptosis and immune regulation, while late changes centered on cell survival, growth and migration. A common regulation of stem cell-associated pathways including Wnt/b-catenin signaling was revealed in dysplastic as well as eHCC potentially predisposing tumor progression. Moreover, we identified 101 genes with significant methylom changes in dysplastic and cancerous lesions with concomitant progressive gene expression alterations in cancer tissue. We further defined an epi-panel of early epigenetic marks in dysplastic lesions including selected CpG-sites with confirmed differential methylation 
in cancer tissue and consequential transcriptional alterations of the target genes using an independent cohort of 362 HCC and 49 surrounding liver samples. Unsupervised hierarchical clustering confirmed a robust classification in malignant and non-malignant lesions.

Conclusion: Our results confirm that epigenetic changes occur early during hepatocarcinogenesis. Epigenetic modifications, therefore, might be of high diagnostic/predictive utility for the identification of dysplastic lesions at risk for cancer progression. The identified (epi-) panel of oncogenic epigenetic marks might be useful to complement phenotypic classifications and facilitate selection of lesions amenable to early therapeutic interventions.

\section{PS013 \\ Klinische Fallvorstellung: Hepatosplenisch T-Zell Lymphom mit chronischer entzündlichen Darmerkrankung unter immunsuppressive Therapie}

\section{Ahmed Afifi; Rosa Rosania; Ali Canbay; Ulrike von Arnim \\ Universitätsklinikum Magdeburg, Gastroenterologie und Hepatologie, Magdeburg, Germany}

Anamnese: Ein 19-jähriger Patient bekannt mit M. Crohn seit 2012 ist in unserer Klinik in April 2018 mit kolikartigen Bauchschmerzen, Gewichtsverlust von $7 \mathrm{~kg}$ und Verschlechterung seiner Diarrhoe vorgestellt worden. Seit der Erstdiagnose wurde er mit Mesalasin und Azathioprin behandelt. Die Therapie wurde in Dezember 2017 bei fehlendem klinischen Ansprechen auf Infliximab umgestellt. Der Patient erhielt seitdem 2 Infusionen mit $300 \mathrm{mg}$ Infliximab.

Körperliche und laborchemische Untersuchung: Klinisch zeigte sich ein 19 jähriger, untergewichtiger (BMI 18) Patient mit einer Hepatosplenomegalie und einen perianalen Abszess. Laborchemisch sahen wir erhöhte Entzündungswerte CRP $(58,2 \mathrm{mg} / \mathrm{l})$, erhöhte LDH $(5 \mu \mathrm{mol} / \mathrm{s} . \mathrm{l})$, Bilirubin $(61.6 \mu \mathrm{mol} / \mathrm{l})$, eine normozytäre Anämie $(\mathrm{Hb} 7,7$ $\mathrm{mmol} / \mathrm{l}$ ) und eine Thrombozytopenie (Thrombozyten $134 \mathrm{Gpt} / \mathrm{l}$ ) bei normaler Leukozytenzahl.

Bildmorphologische, endoskopische und histologische Befunde: In der Sonographie des Abdomens und Darmsonographie sahen wir eine Hepatosplenomegalie und eine entzündliche Aktivität im Dünndarm. Im CT-Abdomen und Thorax zeigten sich entzündliche Verdickung des terminalen lleums mit lokaler reaktiver Lymphadenopathie $(1,2 \mathrm{~cm})$, eine Splenomegalie und Hepatomegalie. Das MR-Sellink zeigte eine multisegmentale entzündliche Wandverdickung im distalen Jejunum, lleum, Zökum und Kolon descendens. In der ÖGD konnte eine Beteiligung des oberen Gastrointestinalentraktes ausgeschlossen werden. Die Koloskopie zeigte ulzerative Veränderungen der lleozökalklappe und vereinzelte Ulzera im lleum, Zökum, Kolon descendens und Rektum. Zur weiteren Abklärung der erhöhten Serum-Bilirubin und Hepatosplenomegalie erfolgte eine Leberbiopsie. Histologisch zeigte sich eine signifikante sinusoidale Dilatation und eine Infiltration durch T-Zellen. In der Knochenmarkaspiration konnte eine ausgeprägte Hyperzellularität als Beweis für eine myeloproliferative Neoplasie gesehen werden. Die weiteren histologische und genetische Untersuchungen der Leberbiopsie und des Knochenmarks konnten die Diagnose eines hepatosplenischen Gamma / Delta-T-Zell-Lymphoms der Stufe IVb stellen.

Verlauf und Zusammenfassung: Das hepatosplenische T-Zell-Lymphom (HSTCL) ist eine aggressive extranodale Form des non-Hodgkin-Lymphoms, die durch sinusoidale Infiltration der Leber, Milzparenchym, und Knochenmark mit Zytopenie und fehlender Lymphadenopathie charakterisiert ist. Es ist eine seltene und aggressive Krankheit, oft mit schlechter Prognose. Mit der zunehmenden
Anzahl von berichteten Fällen von HSTCL bei Patienten unter Biologika und /oder Immunmodulatoren bei chronisch entzündlichen Darmerkrankungen scheint es einen Zusammenhang zwischen der Verwendung dieser und der Entwicklung von HSTCL zu geben. Bei Berücksichtigung der Seltenheit der Fälle (HSTCL) und der vielfältigen Komplikationen der unkontrollierten Erkrankungen (CED) überwiegt jedoch der Nutzen einer indizierten Behandlung das Risiko.

\section{PS014 \\ Cluster of differentiation 44 (CD44) promotes osteosarcoma progression in mice lacking tumour suppressor Merlin}

Monika Hartmann; Jens U. Marquardt; Janina Klemm

Universitätsklinikum Mainz, I. Medizinische Klinik und Poliklinik, Mainz, Germany

Background: Distant metastasis is the leading cause of disease-related death among osteosarcoma patients. How cancer cells become metastatic and spread from the blood stream into secondary sites is still not fully understood. Studies in mice indicate that loss of the tumour suppressor Merlin (Moesin-Ezrin-Radixin-like protein) triggers highly metastatic osteosarcoma. Merlin is a multifunctional protein encoded by NF2 (neurofibromatosis type 2) gene. Analyses in cell culture suggest that Merlin establishes its tumour suppressor activity, at least in part, by forming an inhibitory complex with the cell surface protein "cluster of differentiation 44" (CD44). Consistently, numerous $\mathrm{NF} 2$ mutations in cancer patients are predicted to disturb the interaction of Merlin with CD44. We hypothesised that upon loss of Merlin, the complex with $\mathrm{CD} 44$ would not form releasing $\mathrm{CD}_{44}$ for putative tumour- or metastasis-promoting functions.

Methods: To evaluate the relevance of Merlin-CD44 interaction in vivo, we disrupted the $\mathrm{Cd} 44$ gene and tested the effect on spontaneous tumour growth and progression in mice carrying a mutation in one $\mathrm{Nf}_{2}$ allele. Subsequently, primary osteosarcoma cells were isolated from $\mathrm{Nf}_{2}$-mutant mice and $\mathrm{Cd}_{44}$ expression was manipulated using CRISPR-Casg and lentiviral constructs. These osteosarcoma cells with manipulated $\mathrm{CD}_{44}$ expression were subjected to in vitro and in vivo assays addressing cancer relevant processes. Moreover, we analysed expression profiles of $\mathrm{Cd}_{4} 4$ and $\mathrm{Nf}_{2}$ in a panel of 23 human osteosarcoma samples of primary and metastatic origin from publically available database.

Results: Compared to wild-type mice, heterozygous Nf2-mutant mice developed osteosarcomas, fibrosarcomas as well as hepatocellular carcinomas at increased frequencies. In contrast to $\mathrm{Cd}_{44}$-positive $\mathrm{Nf}_{2}$ mutant mice, none of the $\mathrm{Cd} 44$-negative mice analysed developed hepatocellular carcinomas. Importantly, while the absence of $\mathrm{Cd}_{44}$ gene had no effect on the frequency of osteosarcoma generation, it strongly diminished osteosarcoma metastasis formation in the $\mathrm{Nf}_{2}$ mutant mice. In vitro assays identified adhesion and transendothelial migration as the most prominent cellular phenotypes dependent on CD44. These properties correlated with an increased potential of Cd44-positive (compared to $\mathrm{Cd} 44$-negative) osteosarcoma cells to form lung metastases upon tail vein injection in vivo. The shortest standard isoform of $\mathrm{CD} 44$ was identified as sufficient to reconstitute the transendothelial migration and metastatic potential of $\mathrm{Cd} 44$-negative osteosarcoma cells. Analysis of publically available expression datasets revealed that increased expression of CD44 and downregulation of NF2 might also promote metastasis of human osteosarcoma.

Significance: Our results strongly suggest that in the absence of Merlin, CD44 plays a tumour and metastasis promoting role. Osteosarcoma cells may profit from the expression of $\mathrm{CD}_{44}$ in order to colonise distant sites. 


\section{PS015}

Eine einzelne Fast Food Binge-Eating Episode beeinflusst das Gallensäureprofil, hat jedoch keinen Einfluss auf das Darmmikrobiom

Svenja Sydor'; Chiara Wenning ${ }^{2}$; Sophia Assmuth ${ }^{2}$; Insa Messerschmidt ${ }^{2}$; Jan Best'; Susanne Brodesser ${ }^{3}$; Ramiro Vilchez-Vargas'; Guido Gerken²; Ali Canbay'; Lars P. Bechmann ${ }^{1}$

'Otto-von-Guericke Universität Magdeburg, Klinik für Gastroenterologie, Hepatologie und Infektiologie, Magdeburg, Germany; ${ }^{2}$ Universitätsklinikum Essen, Klinik für Gastroenterologie und Hepatologie, Essen, Germany; ${ }^{3}$ Universitätsklinikum Köln, CECAD Research Center, Köln, Germany

Hintergrund: Die nichtalkoholische Fettlebererkrankung (NAFLE) zeigt weltweit, insbesondere in Industrieländern eine steigende Prävalenz. Eine inaktive Lebensweise und unausgewogene kalorienreiche Ernährung sind mit Fettleibigkeit und dem metabolischen Syndrom, das die NAFLE fördert, verbunden. Die westliche Ernährung umfasst Fast- oder Junk-Food und ist eine schnelle Alternative zu hausgemachten Speisen mit einem hohen Anteil an gesättigten Fettsäuren, Zucker, Salz und Kalorien. Fast Food steht im Zusammenhang mit einer steigenden Prävalenz von Fettleibigkeit und dem metabolischen Syndrom, das häufig im Übermaß konsumiert wird, und den bevorzugten Nahrungsmitteltyp mehrerer Essstörungen wie Bulimie oder dem Binge-Eating Syndrom darstellen. Diese Essstörungen wurden bereits im Zusammenhang mit der NAFLE oder auch dem akuten Leberversagen beschrieben. Bislang ist unklar, welche Auswirkungen eine übermäßige Nahrungsaufnahme auf die akute und chronische Schädigung der Leber hat. Ziel dieser Studie ist es, die Auswirkungen einer einzelnen Fast Food Binge-Episode auf die Leber, den Gallensäuremetabolismus und das Darmmikrobiom zu untersuchen.

Methoden: Es wurden 25 gesunde Personen eingeschlossen und Baseline Serumwerte von Transaminasen, Zelltodmarkern, primären und sekundären Gallensäuren sowie das Lipidprofil gemessen. Zur Quantifizierung der hepatischen Steatose, führten wir eine transiente Elastographie (Fibroscan) durch und bestimmten den Controlled Attenuation Parameter (CAP). Später an diesem Tag erhielten die Teilnehmer eine kalorienreiche Fast Food Mahlzeit ihrer Wahl und wurden aufgefordert, zwei Stunden lang oder bis zur vollständigen Sättigung weiter zu essen. Wir wiederholten alle oben genannten Untersuchungen am folgenden Tag und sammelten vor und nach dem Nahrungsüberschuss Stuhlproben für die Untersuchung des Mikrobioms

Ergebnisse: Am Tag nach der Mahlzeit stieg der CAP geringfügig an, während die Gesamtmenge der Nüchtern-Gallensäuren einen kräftigen Anstieg ohne Veränderungen der FGF-19-Werte zeigte. Die Serumspiegel der primären und sekundären Gallensäuren zeigten eine Erhöhung, aber interessanterweise war die Konzentration der Gallensäure Tauro-Lithocholsäure (TLCA) nach der Fast Food Episode gesunken. Serumtransaminasen, Cholesterin- und Bilirubinspiegel waren am Tag nach der Fast Food Mahlzeit signifikant niedriger. Zelltodmarker (M65, M30) und inflammatorische Zytokine (TNFalpha, IL1-beta) zeigten keine signifikanten Unterschiede. Die Ergebnisse der Mikrobiom-Analyse wiesen keine Unterschiede vor und nach der Fast Food Episode auf.

Schlussfolgerung: Die Einnahme einer einzigen überschüssigen Fast Food Mahlzeit führt zu einem robusten Anstieg der Gesamt-Gallensäuren im Serum und zur Abnahme von Serum-Bilirubin, Transaminasen, Cholesterin und der Gallensäure TLCA 12 Stunden nach der Mahlzeit, was auf eine Beeinflussung von Transport und Homöostase der Gallensäuren schließen lässt.
PS016

Entzündungsparameter im Aszites bei Leberzirrhosepatienten neue potentielle Biomarker zur Diagnose der spontan bakteriellen Peritonitis?

Sophia Scherm; Marika Haderer; Elisabeth Aschenbrenner; Kirstin Pollinger; Sophie Schlosser; Claudia Kunst; Martina Müller-Schilling

Universitätsklinikum Regensburg, Klinik und Poliklinik für Innere Medizin I, Regensburg, Germany

Einleitung: Die spontan bakterielle Peritonitis (SBP) ist eine schwere Komplikation der Leberzirrhose mit einer 1-Jahres-Mortalität von 93\%. Die Pathogenese ist noch nicht vollständig verstanden, jedoch spielt die Translokation von Darmbakterien in die mesenterialen Lymphknoten und die Aszitesflüssigkeit eine wichtige Rolle. Gemäß der S3-Leitlinie "Aszites, spontan bakterielle Peritonitis, hepatorenales Syndrom" erfolgt die SBP-Diagnose anhand des Nachweises von $>\mathbf{2 5 0}$ polymorphonuklearer (PMN) Zellen pro $\mu$ l Aszites. Da eine sofortige Antibiose essentiell ist, müssen dringend neue molekulareParameter identifiziert werden, die eine SBP-Früherkennung ermöglichen.

Ziel: Ziel unsererArbeit war deshalb die Identifizierung neuer Biomarker im Aszites von Leberzirrhosepatienten zur SBP-Früherkennung.

Methoden: Aszitesproben von Leberzirrhosepatienten wurden asserviert. Die Proteinmengen der potentiellen Biomarker Lactoferrin, C3a, IP-10, IL-6, IL-8 und IL-10 wurden mittels ELISA, der Gesamtproteingehalt mittels BCA-Test bestimmt.

Ergebnisse: 69 Aszitesproben von 41 Patienten (Alter 23-77 Jahre; 78\% männlich, $22 \%$ weiblich; $58,5 \%$ äthyltoxisch, $19,5 \%$ kryptogen, $7,3 \%$ nutritiv-toxisch, 4,9\% viral, 2,4\% autoimmun, 2,4\% PBC, 2,4\% nicht-alkoholische Steatohepatitis, 2,4\% BuddChiari Syndrom) wurden untersucht. In 8 Proben $(11,6 \%)$ lag eine gesicherte SBP-Diagnose vor. In 13 Proben $(18,8 \%)$ lag die PMN-Zellzahl bei $>100$ pro $\mu$ l. Die Lactoferrinspiegel bei SBP (1006 ng/ml) waren gegenüber nicht-SBP-Proben (75 $\mathrm{ng} / \mathrm{ml}$ ) stark erhöht. Verlaufsproben ergaben, dass die Lactoferrinmenge bereits im SBP-Frühstadium ansteigt und mit dem Krankheitsverlauf bzw. der Therapieantwort korreliert. Die C3a-Spiegel warenim Vergleich zu nicht SBP-Proben ( $974 \mathrm{ng} / \mathrm{ml}$ ) bei SBP $(511 \mathrm{ng} / \mathrm{ml}$ ) verringert und korrelierten ebenfalls mit dem Krankheitsverlauf. IP-10 sowie relevante Mengen von IL-6, IL-8 und IL-10 wurden in allen Proben nachgewiesen, zeigten jedoch keine Assoziation zum Frühstadium der SBP.

Schlussfolgerung: Lactoferrin und $C_{3}$ a sind molekulare Komponenten des Aszites, deren Menge mit dem Krankheitsverlauf bei SBP assoziiert werden können. Darüber hinaus sind inflammatorische Aktivitäten bei beginnender SBP bereits ab einer Zellzahl von über 100 Granulozyten pro $\mu \mathrm{l}$ nachweisbar. Die Vielzahl weiterer Entzündungsmediatoren und Zytokineunterstreicht die enorme inflammatorische Aktivität im Aszites bei Leberzirrhose, auf deren Basis neue Früherkennungssysteme zur SBP-Diagnose entwickelt werden können. 


\section{PS017}

\section{Proliferation, migration and cell viability in hepatocellular carcinoma - what is the impact of IGFBP2?}

Elisabeth Backu'; Elisabeth Aschenbrenner'; Kirstin Pollinger'; Sophie Schlosser'; Karsten Gülow'; Claudia Kunst'; Martina Müller-Schilling ${ }^{1}$

'Universitätsklinikum Regensburg, Klinik und Poliklinik für Innere Medizin I, Regensburg, Germany

Background: With a worldwide prevalence of 14 million patients, hepatocellular carcinoma ( $\mathrm{HCC})$ is the most common primary malignant neoplasia of the liver (85-90\%) and displays the second highest tumor related mortality, often with poor prognosis upon diagnosis. The insulin like growth factor (IGF) system promotes growth and cell survival via IGF binding to the IGF receptor. Insulin like growth factor binding proteins (IGFBPs) control IGF availability by competitive binding of IGF, therefore limiting proliferative IGF effects. We previously identified IGFBP2 as a target gene of the p53 family, especially of $\mathrm{p73}$, in HCC. These tumor suppressors reply to cellular stress signals by induction of senescence or apoptosis. However, physiologic functions of $\mathrm{p}_{53}$-family induced IGFBP2 in HCC are so far unknown. Thus, effects of IGFBP2 on HCC cell viability, proliferation and migration were evaluated and IGFBP 2 induction by HCC-relevant therapeutics was studied.

Methods: IGF secretion and surface levels of IGF receptors were analyzed in the human HCC cell line Hep3B. Cells were cultured with recombinant IGFBP2 (100 $-1000 \mathrm{ng} / \mathrm{ml})$. Proliferation was measured by flow cytometry, cell viability was determined by MTS assay and cell migration was analyzed using a wound healing model. Activation of the IGF pathway was also determined by milliplex assay. Moreover, effects of HCC-relevant therapeutics on IGFBP 2 and $\mathrm{p}_{73}$ were analyzed by Western blot, qPCR and ELISA.

Results: НерзB cells displayed an intact IGF system, indicated by IGF secretion and surface expression of insulin receptor and IGF receptor 1. Generally, in the presence of IGFBP2 cell viability of Hep3B cells increased in a time-dependent fashion, where most pronounced effects were observed using concentrations between 100 and $500 \mathrm{ng} /$ $\mathrm{ml}$. Highest induction rates in proliferation were detected using IGF$\mathrm{BP} 2$ levels between 100 and $250 \mathrm{ng} / \mathrm{ml}$ with a 13 - and 18 -fold increase at $48 \mathrm{~h}$, decreasing thereafter. Concordantly, IGFBP 2 doses between 100 and $500 \mathrm{ng} / \mathrm{ml}$ resulted in enhanced cell migration. Additionally, treatment with recombinant IGFBP 2 resulted in decreased activation of the IGF pathway. Interestingly, treatment of HepзB cells with bleomycin, doxorubicin and regorafenib caused an induction of both TP73 and IGFBP2.

Conclusion: Although being regarded as growth-limiting factor within the IGF-system, recombinant IGFBP2 exerted proliferative effects on HCC cells. Since an induction of IGF signaling was not observed, the exact mechanisms of action need further elucidation. Nonetheless, $\mathrm{IGFBP}_{2}$ was shown to be a p53 family target gene and was induced by HCC-relevant therapeutics together with the tumor suppressor p73. We therefore hypothesize, that p53-family-mediated mechanisms must exist which redirect IGFBP2-dependent signaling towards growth inhibition. Thus, detailed elucidation of signal transduction on the p73-IGFBP2-axis is indispensable to develop novel diagnostic and therapeutic options for HCC.
PS018

Zelltodinduktion im hepatozellulären Karzinom (HCC) durch Energiedepletion

Marlen Michalski; Elisabeth Aschenbrenner; Kirstin Pollinger; Martina Müller-Schilling; Karsten Gülow

Universitätsklinikum Regensburg, Innere Medizin I, Regensburg, Germany

Hintergrund: Der zelluläre Metabolismus dient nicht allein der Energiegewinnung von Zellen sondern steht auch im engen Zusammenhang mit Signalkaskaden, die Zelltod, Proliferation sowie Differenzierung steuern. Dimethylfumarat (DMF), ein Diester der Fumarsäure mit Methanol wird seit einigen Jahren zur Behandlung von Psoriasis (Fumaderm $^{\oplus}$ ) und Multipler Sklerose (Tecfidera ${ }^{\oplus}$ ) eingesetzt. Wir konnten zeigen, dass DMF in verschiedenen soliden Tumorzelllinien (z.B. PC3, HCT116, MDA-MB231) zu einer erhöhten Glycolyserate und zu einer verminderten mitochondrialen Respiration führt. Durch die Zugabe von DMF könnte somit eine ATP-Depletion erreicht werden, die die Zellproliferation stoppt und Zelltod induziert.

Methoden: Die humanen Lebertumorzellen HepG2 und Huh7 wurden mit DMF (12,5 $\mu \mathrm{M}$ bis $100 \mu \mathrm{M})$ oder 2-Desoxyglukose (2-DG), einen Inhibitor der Glucose-6-phosphat-Isomerase (1,1 mM bis 5,6 $\mathrm{mM}$ für HepG2 und 2,5 mM bis 12,5 mM für Huh7, abhängig von der Konzentration an Glukose im Medium) für bis zu 72 h inkubiert. Die Zelltodinduktion wurde mit Hilfe eines Lumineszenz basierten Viabilitätsassays ermittelt. Die Charakterisierung des Zelltods erfolgte mittels Durchflusszytometrie (FACS) und Doppelfärbung mit DAPI und Annexin V.

Ergebnisse: Die Behandlung mit DMF führte in den humanen Tumorleberzelllinien HepG2 und Huh7 zu einer Dosis- und Zeit-abhängigen Induktion von Zelltod. Die größten Effekte wurden bei einer Konzentration von $100 \mu \mathrm{M}$ DMF und einer Behandlungsdauer von 72 $\mathrm{h}$ beobachtet. Zusätzlich wurden die beiden Zelllinien mit 2-DG behandelt. Dabei konnte die Induktion des Zelltods bereits nach $24 \mathrm{~h} \mathrm{Be}-$ handlungsdauer beobachtet werden. Die größten Effekte für HepG2 konnten mit einer Konzentration von 5,6 mM 2-DG und einer Behandlungsdauer von $72 \mathrm{~h}$ erzielt werden, bei der Zelllinie Huh7 bei einer Konzentration von 12,5 mM 2-DG. Huh7 reagierte stärker auf 2-DG.

Schlussfolgerung: Solide Tumorerkrankungen wie das HCC zeichnen sich durch eine hohe eine Mortalität im Vergleich zu anderen Tumorentitäten aus. Wir konnten zeigen, dass DMF in der Lage ist Zelltod im HCC Zelllinien zu induzieren. DMF ist bereits seit vielen Jahren im klinischen Gebrauch und weist im Gegensatz zu etablierten Krebstherapeutika nur geringe Nebenwirkungen auf. DMF könnte somit als mögliche neue Therapieoption für die Behandlung solider Tumore wie dem HCC dienen.

\section{PS019}

Kenntnisstand über Stuhltransplantation (FMT) in der deutschen Bevölkerung: Bevorzugte Applikationswege und Bedenken gegenüber der Therapie

Tim Lachmund'; Ulrike von Arnim²; Peter Malfertheiner'; Alexander Link ${ }^{1}$

'Otto-von-Guericke Universität Magdeburg, Klinik für Gastroenterologie, Hepatologie und Infektiologie, Magdeburg, Germany; ${ }^{2}$ Otto-von-Guericke University, Magdeburg, Klinik für Gastroenterologie, Hepatologie und Infektiologie, Magdeburg, Germany

Einleitung: Stuhltransplantation (FMT) ist eine empfohlene Therapieoption bei rezidivierenden oder therapierefraktären Clostridioides difficile Infektionen (CDI). Zunehmende Evidenz deutet auf einen potentiellen Nutzen der FMT auch für andere Erkrankungen, wie das 
metabolische Syndrom oder chronisch entzündliche Darmerkrankungen, hin. Es existieren unterschiedliche Applikationswege und Applikationsprotokolle. Mit dieser Arbeit wollten wir die bevölkerungsbezogenen Präferenzen in Hinblick auf die FMT beurteilen. Der Hauptfokus der Untersuchungen lag in der Evaluation der Bereitschaft zur FMT, potenzieller Ängste und Bedenken sowie methodologischen Präferenzen.

Methode: Eine strukturierte Umfrage mittels Fragebögen zur FMT wurde 2014 bis 2015 in Sachsen-Anhalt durchgeführt. Die Zielkohorte beinhaltete Teile der Allgemeinbevölkerung, Patienten sowie Personen mit Bezug zum Gesundheitssystem (Ärzte, Pflegepersonal). Es standen 501 Fragebögen bei einer Rückläuferquote von $98 \%$ für die Analysen zur Verfügung.

Ergebnisse: Im Falle einer CDI würden insgesamt 82,2\% der Probanden eine FMT als Therapie in Betracht ziehen, während nur 4,6\% die FMT als Behandlungsoption ablehnen. 83,4\% der Teilnehmer waren nicht ausreichend über verschiedenen FMT-Applikationswege informiert. Bei $41,7 \%$ der Befragten würde der Applikationsweg eine wichtige Rolle bei der Entscheidung zur FMT spielen. Insgesamt 11,4\% der Teilnehmer würden eine Applikation über eine Sonde (nasojejunal/ gastral), 49,9\% über eine Koloskopie und 63,1\% über Kapseln bevorzugen. Nur $23,5 \%$ der Befragten hatten subjektiv ausreichendes Wissen über die Option verschiedener Spender. 64,3\% der Befragten würden einen Spender aus ihrer Familie/Angehörigen bevorzugen, 58,9\% würden sogar ihren eigenen Stuhl für eine FMT in Betracht ziehen, und nur 20,6\% würden einen unbekannten Spender bevorzugen. Ein mögliches Infektionsrisiko wurde von $45,9 \%$ als größtes Bedenken angegeben.

Zusammenfassung: FMT wird in der deutschen Bevölkerung gut akzeptiert. Es liegt eine hohe Bereitschaft zur Therapie im Erkrankungsfall vor, auch wenn gewisse Bedenken und Unklarheiten hinsichtlich des Applikationsweges und des bevorzugten Spenders existieren. Die Kenntnisse potentieller Sorgen und Bedenken sollen bei der Aufklärung und Durchführung einer FMT berücksichtigt werden.

\section{PS020 \\ Cancer stem cell sub-population drives treatment resistance to anti-angiogenic drugs in hepatocellular carcinoma}

Darko Castven'; Carolin Czauderna'; Diana Becker'; Sharon Pereira'; Marcus A. Wörns'; Snorri S. Thorgeirsson ${ }^{2}$; Peter Grimminger ${ }^{3}$; Hauke Lang ${ }^{3}$; Peter R. Galle'; Jens U. Marquardt ${ }^{1}$

'Universitätsmedizin Mainz, I Med. Klinik, Mainz, Germany; ${ }^{2}$ Center for Cancer Research, National Cancer Institute, NIH, Laboratory of Human Carcinogenesis (LHC), Bethesda, United States; ${ }^{3}$ Universitätsmedizin Mainz, Klinik für Allgemein-, Viszeral- und Transplantationschirurgie, Mainz, Germany

Background and Aims: Hepatocellular carcinoma (HCC) is highly vascular tumor where activation of neo-angiogenic processes during disease progression is frequently associated with poor clinical outcome. Consequently, inhibition of neo-angiogenesis is an effective treatment strategy for advanced HCC. However, development of chemoresistance is observed in the majority of patients. Evidence suggests that cancer stem cells (CSCs) may contribute to the acquisition of resistance in many solid tumors, but their exact role in this process for HCC remains to be defined. Here, we evaluate the importance of CSCs in the development of resistance and relapse formation after exposure to different anti-angiogenic therapies in HCC and define concomitant adaptive molecular changes.

Method: Four HCC cell lines and two primary HCC isolates were exposed to sorafenib and sunitinib for a total of 14 days. The treatment effects on CSCs were estimated by sphere forming capacity in vitro and tumor-initiating potential in vivo, as well as the side-population (SP) approach. Expression of key oncogenic and CSC markers, such as EpCAM, CD133 and ABCG2 transporter, were assessed by qRT-PCR and flow cytometry. Furthermore, whole transcriptome analyses were performed across the cell lines.

Results: Both treatments effectively reduced oncogenic properties in all investigated HCC cells. However, sustained anti-proliferative effect after treatments was observed in only one cell line. In three other lines initial treatment effect was subsequently followed by rapid re-growth thereby mimicking the responses observed in patients. Interestingly, two cell lines showed differential response to applied drugs, showing anti-proliferative effects to sorafenib, while relapse formation occurred after sunitinib treatments. While anti-oncogenic effects in sensitive cell lines were associated with significant reduction in sphere forming and tumor-initiating capacity, CSC marker EpCAM as well as SP cells, resistant cell line showed transient increased in CSC properties. Acquired resistance to both drugs uniformly developed in cell lines suggesting that common molecular mechanisms might be operative. These adaptive molecular changes involved signaling pathways known to be associated to cell survival, proliferation and cell cycle regulation (RAS, AKT, MYC, P53), as well as angiogenesis (VEGFR, PDGFR). Furthermore, the resistant cell lines showed compensatory upregulation of key oncogenic molecules such as EGFR as well as multidrug resistance $A B C$ transporters.

Conclusion: Our model recapitulates features of drug resistance observed in human HCC patients. Resistance to anti-angiogenic therapies might be fueled by transient expansion of CSCs. Therefore, specific targeting of CSCs as well as pro-oncogenic compensatory signaling pathways might be an effective therapeutic strategy to overcome resistance in HCC.

\section{PS021}

Utility of Neutrophil-Lymphocyte Ratio (NLR) and Platelet-Lymphocyte Ratio (PLR) as Cost-effective Diagnostic markers for Tuberculosis in Travellers/Migrants

\section{S. Srikanth; Pillai M. Gopala Krishna}

Amrita Institute of Medical Sciences and Research Centre, Internal Medicine, Kochi, Kerala, India

Background: In developing countries like India and other south Asian countries, the spread of Tuberculosis is very extensive. Travellers from and to these endemic countries will play a major role in spread of the disease. NLR and PLR can serve as cost effective biomarkers for predicting Tuberculosis at an earlier stage and control the disease speared.

Objective: To study the potential role of NLR and PLR as diagnostic markers in travellers/migrants with diabetes who are at risk of developing tuberculosis. Secondary objectives: The sensitivity and prognostic utility of these 2 markers in comparison with the more established markers like CRP and ESR. Also as there is no clear marker available for predicting diabetics who are predisposed and at risk of developing TB. This study will play a great role in predicting TB in travellers or Migrants from endemic areas as most of them are migrants.

Methods: A combined Retrospective+ prospective study on NLR and PLR in 3 groups 1) Healthy controls,2) Type 2 diabetes patient with TB 3) Type 2 diabetes patient without TB70 cases were taken in each group and 70 controls were taken and data obtained were analysed

Result and Discussion: In our study, we found that NLR and PLR were highly significant with a P value of 0.001 as compared to the CRP with 
P value 0.001. According to Stevenson et al. 15\% of TB burden in India was attributed to diabetes. Diabetics is a major cause of conversion of latent to active TB, reactivation of old TB. MPV was not statistically significant to be used as a marker

Conclusion: Large number of Diabetic population who are at risk of developing TB during travel to endemic countries. The cases of TB can be easily identified even at Primary Health Care Centre with just a peripheral smear from which NLR and PLR can be calculated and need not depend on costly investigation. This will reduce the financial burden of the travels. It will help in early identification of diseases among travellers and also to take necessary precaution before travel

Key Words: NLR, PLR, Tuberculosis

\section{PS022 \\ Mean Platelet Volume (MPV) and Red Cell Distribution Width (RDW) as an early Predictive Markers of Pulmonary Embolism in long distance Air Travelers}

\section{S. Srikanth'; Pillai M. Gopala Krishna²}

${ }^{1}$ Amrita Institute of Medical Sciences and Research Centre, Internal Medicine, Kochi, Kerala, India; ${ }^{2}$ Amrita Institute of Medical Sciences and Research Centre, Affiliated to Amrita Vishwa Vidyapeetham, Internal Medicine, Kochi, Kerala, India

Objective: Epidemiological studies indicate that, the risk of venous thromboembolism increases by 2 to 3 folds during long-haul travels in potential risk group travellers. Management of Pulmonary Embolism is extremely difficult especially during air travel and the chances of mortality and morbidity is high. Pulmonary Embolism is preventable disease. The mortality can be highly reduced if identified at right time and precautionary measures are taken. There is no biomarker which can predict Pulmonary Embolism in quick and cost effective way. MPV and RDW are bio markers which can aid in early identification and effective management of Pulmonary Embolism.

Method: We conducted a retrospective study by screening more than 2.5 lakh patients who presented to the hospital during 2013 to 2018 (6 Years) and identified 300 cases of Pulmonary Embolism. The patient's MPV and RDW values were analysed along with other details like demographic details and comorbid conditions. Statistical data analysis was done using SPSS Tool.

Results: The result showed male predominance and the mean age of the study group was 54 years. Major Comorbid conditions were Dyslipidaemia and Diabetics. $53 \%$ of the study population were alcoholics and smokers. Mean Value analysis of the MPV and RDW values showed a very high statistical significance as biomarkers (with the ' $p$ ' value $<0.001$ ). ROC analysis for specificity and sensitivity for MPV and RDW indicated high correlation with area under the curve coverage of $83 \%$ and $79 \%$ respectively. MPV value of 12 and RDW of 15 taken as cut off and increase in their value by $5 \%$ or more indicate the potential condition of Pulmonary Embolism.

Conclusion: MPV and RDW are novel and cost effective biomarkers. It is non-invasive, can easily be estimated from a complete blood count. Require no special lab setups or specialist to interpret the values and correlate with potential Pulmonary Embolism incidence. These biomarkers can be checked in the airport medical facility itself. The high risk cases can be identified and necessary precaution can be taken. This aids to bring down the instances of Pulmonary Embolism during air travel.

Key Words: MPV, RDW, Bio Markers, Pulmonary Embolism

\section{PS023}

\section{Kontaktlose Messung der Herzratenvariabilität und} ihre Bedeutung als potentieller geriatrischer Biomarker

Xinchi Yu ${ }^{1}$; Christoph Hoog-Antink'; Joao Batista ${ }^{2}$; Steffen Leonhardt ${ }^{1}$; Cornelius Bollheimer ${ }^{2}$; Thea Laurentius ${ }^{3}$

${ }^{1}$ Helmholtz-Institut für Biomedizinische Technik der RWTH Aachen, Lehrstuhl für Medizinische Informationstechnik, Aachen, Germany; ${ }^{2}$ Uniklinik RWTH Aachen, Lehrstuhl für Altersmedizin, Aachen, Germany; ${ }^{3}$ Lehrstuhl für Altersmedizin, RWTH Aachen, Franziskushospital Aachen, Klinik für Innere Medizin und Geriatrie, Aachen, Germany

Frailty ist als zentrales geriatrisches Syndrom mit einer verminderten Belastbarkeit gegenüber internen und externen Stressoren assoziiert und ist ein Prädiktor für eine erhöhte Morbidität und Mortalität. Frailty soll nach einer Pilotstudie mit einer geringeren Herzratenvariabilität vergesellschaftet sein (Katayama: Cardiac autonomic modulation in non-frail, pre-frail and frail elderly women: a pilot study, 2015).

Aktueller Goldstandard ist die Messung der Herzratenvariabilität mittels Langzeit-EKG. Diese Notwendigkeit des Aufbringens von Elektroden auf die Haut ist insbesondere für geriatrische (delirgefährdete) Patienten mit Problemen in der Akzeptanz verbunden. Daher wurde im Rahmen einer Entwicklungsarbeit an der Schnittstelle zwischen Technik und Geriatrie ein kontaktloses Verfahren zum Messen der Herzratenvariabilität entwickelt. Methodisch liegt dabei das Verfahren des Photoplethysmographie-Imaging (PPGI) zugrunde, welches die kontaktlose und kamerabasierte Weiterentwicklung der Photoplethysmographie (bekannt in der sog. Messtechnologie via "Fingerclip") darstellt und somit eine kontaktlose Erfassung des Pulswellensignals ermöglicht.

Zur Evaluation, ob tatsächlich relevante Unterschiede der Herzratenvariabilität bei Vorliegen des Frailty-Syndroms existieren, erfolgte zunächst die vergleichende Bestimmung bei gesunden Probanden ab 70 Jahren und bei geriatrischen Patienten mit Vorliegen einer Frailty ab 70 Jahren, welche in die sogenannte geriatrische frührehabilitative Komplextherapie eingeschlossen waren. Frailty wurde nach den Fried-Kriterien klassifiziert und umfasste ungewollten Gewichtsverlust, Ganggeschwindigkeit, Handkraft, subjektive Erschöpfung sowie physische Aktivität. Die gesunden Probanden wurden einmalig gemessen. Dabei wurde die kontaktlose Messung der Herzratenvariabilität bei den Probanden zunächst während einer zehn-minütigen Ruhephase in liegender Position durchgeführt. Anschließend erhielten die Probanden eine Physiotherapieeinheit zur Anregung des Herzund Kreislaufsystems, bevor eine Folgemessung über 15 Minuten in liegender Position durchgeführt wurde. Die geriatrischen Patienten wurden zu Beginn der geriatrisch frührehabilitativen Komplextherapie und ein zweites Mal nach Minimum 7 Tage Abstand erneut gemessen. Als Referenz für die kontaktlos gemessene Herzratenvariabilität wurde parallel das EKG eines Patientenmonitors aufgezeichnet.

Wir konnten im Rahmen der Pilotphasen zeigen, dass die kontaktlose Messung der Herzratenvariabilität mittels PPGI prinzipiell möglich ist. Beim Vergleich der gesunden Probanden mit den geriatrischen Patienten fanden sich wenig Unterschiede, aber es zeigt sich eine Besserung der Herzratenvariabilität der geriatrischen Patienten zu Beginn und am Ende der geriatrisch frührehabilitativen Komplextherapie.

Katayama: Cardiac autonomic modulation in non-frail, pre-frail and frail elderly women: a pilot study, 2015 


\section{PS024}

\section{Erste Darstellung eines idiopathischen Mastzellaktivierungs- syndroms (MCAS) bei einem Jugendlichen: Assoziation mit Histamin- und Salizylatunverträglichkeit sowie eine wichtige Differentialdiagnose bei gastroenterologischen Problem- patienten}

Martin Raithel'; Tobias Rechenauer ${ }^{2}$; Thomas Götze ${ }^{2}$; Gregor Siebenlist ${ }^{2}$; Aline Rückel ${ }^{2}$; Hanns-Wolf Baenkler'; Arndt Hartmann"; Florian Haller'; André Hörning ${ }^{2}$

'Waldkrankenhaus St. Marien Erlangen, Medizinische Klinik II, Erlangen, Germany; ${ }^{2}$ Universitätsklinikum Erlangen, Pädiatrie, Erlangen, Germany; ${ }^{3}$ Universitätsklinikum Erlangen, Medizinische Klinik ${ }^{3}$, Erlange, Germany; ${ }^{4}$ Universitätsklinikum Erlangen, Pathologie, Erlangen, Germany;

Hintergrund: Chronische Bauchschmerzen sind eine häufige Ursache für medizinische Konsultationen und können mit anderen unspezifischen Symptomen assoziiert sein. Auch wenn keine organische Ursache bei der Routineuntersuchung offensichtlich ist, kann sich manchmal eine gründlichere Untersuchung wegen der zunehmenden Anzahl von Mastzellaktivierungserkrankungen und/oder Gl-Allergie lohnen.

Falldarstellung und Diagnostik: Ein 13-jähriger Jugendlicher wird mit einer Anamnese von krampfartigen Darmschmerzen, Übelkeit, Schwindel, Kopfschmerzen, Schlaflosigkeit und Hitzewallungen seit $>2$ Jahren vorgestellt. Der Familie war nicht bewusst, dass die Symptome mit einer bestimmten Diät verbunden waren.

Viele Untersuchungen (Laboranalyse, Zöliakie, Stuhluntersuchung, BKS, CRP, fäkales Calprotectin, Allergie-Check, Abdominal-, Herz-UItraschall, $\mathrm{H}_{2}$-Atemtest, EEG, kraniale MRT-Bildgebung etc) waren ohne pathologische Befunde.

Bei der früheren ÖGD wurde eine geringfügige unspezifische Gastritis gefunden und mit PPI ohne Verbesserung der Symptome behandelt.

Ein Symptomtagebuch zeigte einen Zusammenhang mit dem Verzehr von Käse und Schokolade, was möglicherweise auf eine Histaminintoleranz hindeutete. Eine Salicylat-Intoleranz wurde durch einen funktionellen Eicosanoid-Test diagnostiziert, der eine erhöhte Freisetzung der Leukotriene feststellte. Obwohl die Tryptase normal war, wurde ein erhöhter Serum-TNFa gefunden (27,7; normal $<8,1)$ sowie eine signifikant erhöhte Ausscheidung von Methylhistamin (17,8 normal $<6,5$ ), was auf eine hohe endogene Histaminproduktion hindeutete. Eine neue problemorientierte Endoskopie mit Immunhistochemie und endoskopischer Lavage zeigte noduläre hyperplastische Lymphfollikel im terminalen lleum. Immunhistochemisch wurden bis zu 60 Gewebemastzellen/HPF ( $<30 / H P F)$ mit normaler Morphologie nachgewiesen. Eine endoskopische Darmlavage am unteren GI-Trakt konnte keine lokale IgE-Sekretion nachweisen, so dass eine lokale Typ I GI-Allergie ausgeschlossen werden konnte. Die Histaminabbaufähigkeit des Gewebes war jedoch ex vivo verringert.

Zusammenfassung: Da alle diagnostischen Kriterien für das idiopathische MCAS (erhöhte Mediatorensekretion bei erhöhter Mastzellzahl) erfüllt wurden, erhielt der Patient eine Ernährungsberatung, um histamin- und salicylatreiche Nahrungsmittel zu vermeiden. Zudem wurde oral mit Histaminrezeptorblockern, einem Leukotrienantagonisten und Vitamin $\mathrm{C}$ behandelt.

Unter diesem Regime erlebte der Patient eine langfristige deutliche Verbesserung der Beschwerden und gilt als erster Jugendlicher mit dokumentiertem MCAS.
PS025

Biologische Therapie der NSAID-Intoleranz und Nahrungsmittelallergie mit Eiern des Schweinepeitschenwurm Trichiuris suis (TSO) in Verbindung mit Antiallergika

\section{Johanna Heinrich'; Katharina Hotfiel'; Ralf Rieker²; Stefanie Finzel ${ }^{3}$; Detlef} Goj ; Martin Raithel

\author{
'Malteser Waldkrankenhaus St. Marien Erlangen, Medizinische Klinik II, \\ Erlangen, Germany; ${ }^{2}$ Uniklinikum Erlangen, Pathologie, Erlangen, Germany; \\ ${ }^{3}$ Uniklinikum Freiburg, Rheumatologie und Klinische Immunologie, \\ Freiburg, Germany; ${ }^{4}$ TSO Laboratories Inc, Hamburg, Hamburg, Germany; \\ ${ }^{5}$ Waldkrankenhaus St. Marien Erlangen, Medizinische Klinik II, Erlangen, \\ Germany
}

Eine verminderte Stimulation des Immunsystems mit bakteriellen/ parasitären Antigenen aufgrund zunehmender Hygiene wird in der westl. Welt mit dem Anstieg von Allergien, chron. entzündlichen Erkrankungen und Autoimmunopathien assoziiert. Während viele dieser Erkrankungen mit Steroiden/Immunsuppressiva behandelt werden, finden sich auch Therapieberichte zum Einsatz von Würmern bei diesen Erkrankungen.Dieser Fall berichtet von einer 28J Pat mit Samter-Trias (chron. Sinusitis, Rhinorrhoe, Asthma), schwerer Obstipation mit Bauchkoliken, manifesten Intoleranzreaktionen auf Salizylate, Probiotika und Reaktionen auf Nahrungsmittel inkl. Histamin (Flush, Juckreiz, Kopfschmerzen). Vor Diagnosestellung wurde die Pat anlässlich eines Lupus erythematodes mit NSAR behandelt und erlitt rezidivierend die oben beschriebenen Symptome. Trotz NSAR-Karenz und ASS-Desaktivierung (10omg) blieben eine schwere Obstipation, rez. Kopfschmerzen, Flush und viele andere Krankheitssymptome bestehen. Da die Pat.keine der verfügbaren Probiotikaformulationen vertragen konnten, entschied sie sich für eine biologische Therapie mit TSO, der auch beim M. Crohn eingesetzt wurde. Der Einsatz von Helminthen wird mit der Induktion von regulatorischen T-Zellen und -Mechanismen sowie einer möglichen Änderung der Mikrobiota in Verbindung gebracht.Immunhistochemisch zeigte sich im Duodenum und unteren GIT eine Mastzellhyperplasie, Eosinophilie und geringe Colitis vor TSO-Therapie. Unter Einnahme von 2500 TSO alle 14 Tage konnten koloskopisch vitale Würmer nachgewiesen werden. Unter einer definierten Antiallergikatherapie mit H1-Blocker, Cromoglycinsäure und ASS-Desaktivierung konnte sofort eine Rückbildung der schweren Obstipation und Bauchschmerzen erreicht werden. Nach ca. 10 Wochen kam es zu einer ansteigenden Toleranz von Salizylaten, Rückgang der Rhinorrhoe und Sinusitis sowie Verbesserung der Asthma- und Fatiguesymptomatik. Insg. zeigte sich eine deutliche Rückbildung des Erlanger Score für mastzellbedingte Beschwerden von 41 auf 16 Punkte (normal < 6) innerh. von 6 Monaten. Bezüglich des Lupus erythematodes konnte nur eine geringe Besserung der Krankheitsaktivität festgestellt werden.Obwohl Wurmtherapien in systematischen Studien (z.B. CED) keine Zulassung erhalten haben, könnte diese biologische Therapieform bei individuellen Personen, bestimmter Prädisposition und einzelnen Krankheitsbildern einen Benefit zeigen. Bei dieser Pat. mit komplexem Krankheitsbild ist die Rückbildung der Obstipation, der Samtertrias und des Asthmas eindrucksvoll. Insofern sollten zukünftige Studien zeigen inwieweit TSO bei Mastzellüberaktivität, Mutationen der Histaminabbauenzyme (NSAID-Intoleranz) und Immunstörungen den Anteil regulatorischer T-Zellen, protektiver Zytokine oder von protektiven Mikrobiotaveränderungen beeinflussen. In Anbetracht der zunehmenden Anzahl von Allergien und mastzellbedingten Erkrankungen zeigt dieser Fall eine potentiell bedeutsame,nicht-immunsuppressive Therapiealternative auf. 


\section{PS026}

\section{Extracellular DNA drives cholesterol crystal embolism-related tissue infraction}

Chongxu Shi'; Shrikant R Mulay ${ }^{1}$; Helen Liapis ${ }^{2}$; Barbara Klinkhammer ${ }^{3}$; Peter Boor ${ }^{3}$; Hans-Joachim Anders ${ }^{1}$

'Ludwig-Maximilians-Universität München, Medizinische Klinik und Poliklinik IV, München, Germany; ${ }^{2}$ Washington University, Department of Pathology and Immunology School of Medicine, Washington, American Samoa; ${ }^{3}$ RWTH Aachen University, Aachen, Department of pathology, Achen, Germany; ${ }^{3}$ RWTH Aachen University, Aachen, Department of pathology, Aachen, Germany

Cholesterol crystal (CC) embolism may be an under-recognized cause of acute kidney injury (AKI), especially of catheter intervention- or major surgery- related AKI. Little is known about the pathophysiology of CC embolism because of lack of animal models. Injecting CC into the left renal artery of $\mathrm{C}_{57} \mathrm{BL} / 6 \mathrm{~N}$ mice caused a sudden drop in glomerular filtration rate (GFR) associated CC embolism and crystal clot formation leading to either partial CC or complete occlusion of numerous interlobar arcuate, and interlobular documented by histology and $3 \mathrm{D}$ reconstructions of vascular contrast micro-CT scans. $24 \mathrm{~h}$ after CC injection partial obstruction was associated with ischemic necrosis of respective tubular S3 segments, while complete obstruction caused large territorial infarction. Such infarcts were surrounded by massive neutrophil infiltrates apparently also derived from vessels of the renal capsule (rim sign). Necroptosis is known to drive post-ischemic AKI, and lack of Mlkl had partially effect on infarct size and tubular injury but no effect on GFR loss. Obstructed artery quantification showed that Mlkl deficiency had no effect on crystal clot obstruction. Crystal clots stained positive for platelets, neutrophils, fibrin, and DNA. While neutrophil depletion only partially attenuated GFR loss and infarct size recombinant DNase I was fully protective as it reduced the number of complete vascular occlusion. In vitro studies revealed that CC induce membrane rupture and DNA release from endothelial cells and neutrophils and that CC-induced activation of platelets induces neutrophil extracellular traps formation. CC embolism was found in 446 of 92,000 diagnostic kidney biopsies (0.5\%). Thus, CC embolism activates numerous cell types to release DNA, which is a central component of crystal clot formation, vascular occlusion, and kidney infarct-related AKI.

\section{PS027 \\ Baseline characteristics of HFrEF patients receiving Sacubitril/Valsartan in the German outpatient setting - a subgroup analysis from ARIADNE}

Uwe Zeymer'; Stephan Hupfer ${ }^{2}$; Tobias Merkel ${ }^{2}$

${ }^{1}$ Klinikum Ludwigshafen, Institut für Herzinfarktforschung, Ludwigshafen, Germany; ${ }^{2}$ Novartis Pharma GmbH, Cardio-Metabolic, Nürnberg, Germany

Background: ARIADNE (Assessment of Real-life cAre Describing EuropeaN heart failurE management) was designed as a prospective registry providing a thorough understanding of the demographic and clinical features of symptomatic patients with chronic heart failure with reduced ejection fraction (HFrEF) managed in the outpatient sector across Europe, and the way sacubitril/valsartan (S/V) is introduced by office-based cardiologists and specialised primary care physicians. Here, we present baseline data for the German subgroup.

Methods: Consecutive male or female patients ( $\geq 18 \mathrm{y}$ ) with symptomatic chronic HF (NYHA II-IV) and documented reduced LVEF were eligible for the study. The subgroup of patients recruited in Germany consisted of 4768 patients (main analysis set: $50.5 \%$ Standard of Care
(SoC) vs. $49.5 \%$ S/V. For volatile clinical parameters (e.g. NYHA class, blood pressure and laboratory values), analysis was restricted to the S/V group who had been initiated no longer than 4 weeks ago (3452 patients, $68.3 \%$ SoC vs. $31.7 \%$ S/V).

Results: Mean age at the time of inclusion into the study was statistically different between the two groups with patients receiving S/V being younger ( $S / V 67.8 y \pm 11.6$ vs SoC $69.5 y \pm 11.0 ; p<0.0001)$. The study population divided into $76.2 \%$ of male versus $23.8 \%$ of female patients with no statistical difference between the different treatment groups $(p=0.2934)$. Mean LVEF at inclusion was lower in the S/V group compared to the SoC group (S/V $32.7 \% \pm 8.1$ vs. SoC $36.3 \% \pm 8.5 ; \mathrm{p}$ $<0.0001)$. Assessment of NYHA functional class showed a statistical significant enrichment in NYHA III (S/V $57.1 \%$ vs. SoC $32 \%$ ) and NYHA IV (S/V $3.1 \%$ vs. SoC $1.4 \%$ ) in the S/V group, whereas patients in the SoC group displayed enrichment in NYHA II (S/V $39.8 \%$ vs. SoC 66.5 $\%)$. Analysis of the medical history revealed a higher burden of comorbidities in S/V as compared to SoC. Statistical significant differences were seen for example in the percentage of patients with atrial fibrillation (S/V $41.2 \%$ vs. SoC 37.9\%; $\mathrm{p}=0.0205$ ), coronary heart disease (S/V $59.1 \%$ vs. SoC $63.1 \% ; \mathrm{p}=0.0041$ ), and renal disease due to hypertension (S/V $9.3 \%$ vs. SoC $6.6 \% ; p=0.0004$ ).

Specification of heart failure treatment (in \% of patients on current SoC HF treatment, S/V treatment started no longer than 4 weeks ago) showed a higher usage of MRAs (S/V $57.1 \%$ vs. SoC $51.7 \% ; p=0.0058$ ) and loop diuretics (S/V $63.1 \%$ vs. SoC $57.6 \% ; p=0.0045$ ) in the S/V group. Usage of calcium channel blockers (S/V $6.7 \%$ vs. SoC $10.8 \%$; $\mathrm{p}=0.0004$ ) was less frequent in the $S / V$ group .

There was no statistical difference in the frequency of beta blockers, Ivabradine, Digitalis and diuretics other than loop diuretics.

Conclusion: The presented German subgroup data of ARIADNE offer insights into the prescription patterns in the German outpatient real-world setting. S/V use in Germany seems to be influenced by age, disease severity (e.g. NYHA class) and comorbidities.

\section{PS028}

Peripheral edema and headache associated with amlodipine treatment: a meta-analysis of randomized, placebo-controlled trials

Davor Vukadinovic'; Sean Scholz'; Michael Böhm²; Felix Mahfoud ${ }^{2}$

'Universitätsklinikum Homburg, Innere Medizin III - Kardiologie, Angiologie und internistische Intensivmedizin, Homburg, Germany; ${ }^{2}$ Universitätsklinikum des Saarlandes und Medizinische Fakultät der Universität des Saarlandes, Klinik für Innere Medizin III, Kardiologie, Angiologie und internistische Intensivmedizin, Homburg, Saar, Germany

Background: Use of amlodipine for treatment of arterial hypertension (AH) and stabile angina pectoris (SAP) is sometimes limited by occurrence of peripheral edema and headache. We aimed to explore the true magnitude of this phenomenon by determining the rate and placebo-adjusted rate of these side effects (SE).

Methods: We performed a meta-analysis by including all randomized, placebo-controlled trials reporting edema and headache with amlodipine in patients with AH and SAP. Placebo-adjusted rate (\%) was determined as follows: (SE amlodipine \% - SE placebo \%) / SE amlodipine \%. Electronic databases PubMed, Web of Science, and Cochrane library were systematically searched for eligible trials published until July 2018. Diseases such as heart failure, severe renal or hepatic disease were not considered. Furthermore, in most of the trials, a wash-out period took place before the study medication was given. 
Results: Data from 7,226 patients of 22 trials were analyzed. In most of the analyzed trials, duration of follow up was comparable, and lasted about 8 weeks. Rate of edema was higher on amlodipine compared with placebo (16.6 vs $6.2 \%$, RR: $2.9,95 \%$ Cl: $2.5-3.36, \mathrm{p}<0.0001$ ). The placebo-adjusted rate was $63 \%$, indicating that $37 \%$ of edema cases were unrelated to amlodipine. Treatment with low/medium doses (2.5-5 $\mathrm{mg}$ ) resulted in lower rates of edema (RR: 2.01, 95\% Cl: 1.41-2.88, $\mathrm{p}=$ o.0001) compared with high dose (10 mg) (RR: $3.08,95 \% \mathrm{Cl} 2.62-3.6, \mathrm{p}<$ $0.0001, p$ for interaction $=0.03$ ). Headache was reduced using amlodipine compared with placebo (7.9 vs $10.9 \%$, RR: $0.77,95 \% \mathrm{Cl}$ : $0.65-0.90$, $p=0.002$ ) and was driven by use of low/medium doses (RR: $0.52,95 \%$ $\mathrm{Cl}: 0.40-0.69, \mathrm{p}<0.00001$ versus RR: $0.92,95 \%-\mathrm{Cl}: 0.74-1.15, \mathrm{p}=0.45$, for high doses, $p$ for interaction $=0.002$ ). Co-administration of renin-angiotensin-inhibitors to low/medium dose of amlodipine resulted in higher BP reductions with equivalent rates of edema in comparison with amlodipine monotherapy ( $p$ for interaction $=0.23$ ).

Conclusion: Patients on amlodipine exhibit a dose-dependent 3 -fold increased risk of peripheral edema compared with placebo. Of note, up to one third of edema cases on amlodipine might not be induced by amlodipine. Headache is reduced on amlodipine treatment, mainly driven by use of this drug at low/medium doses potentially related to better blood pressure control.

\section{PS029 \\ Verbessert eine positive Einstellung zur Chemotherapie die Prognose einer Krebserkrankung? Ergebnisse einer multizentrischen deutschen Therapiestudie bei nicht kleinzelligem Lungenkarzinom.}

Tadeusz Marczewski'; Barbara Ritter'; Jürgen Fischer ${ }^{2}$; Thomas Fink ${ }^{3}$; Martin Wolf $^{1}$

${ }^{1}$ Klinikum Kassel, Klinik für Onkologie und Hämatologie, Kassel, Germa$n y ;{ }^{2}$ SLK Lungenklinik Löwenstein, Onkologie / Krebsheilkunde mit Palliativmedizin, Klinik für Innere Medizin, Löwenstein, Germany; ${ }^{3}$ Rangauklinik Ansbach GmbH, Pneumologie/Allergologie/Onkologie, Ansbach, Germany;

Hintergrund: Vielfältige Faktoren beeinflussen die Überlebenszeit von Patienten mit Krebserkrankungen. Nur wenige Daten liegen zurück zum prognostischen Einfluss der psychischen Einstellung von Patienten gegenüber einer Chemotherapie vor. Methode: Im Rahmen einer multizentrischen deutschen Therapiestudie beim Nichtkleinzelligen Lungenkarzinom wurden Patienten zu ihrer Einstellung gegenüber einer Chemotherapie befragt. Mittels Fragebögen wurde erhoben, für welchen Überlebensgewinn sie bereit wären, Nebenwirkungen einer Therapie zu ertragen. Nachfolgend wurde untersucht, ob die Akzeptanz von Nebenwirkungen einen Einfluss auf die Prognose der Erkrankung hatte. Ergebnisse: In die Therapiestudie wurden insgesamt 272 Patienten aufgenommen. 142 von Ihnen füllten den Fragebogen zur Chemotherapieakzeptanz aus. Circa $80 \%$ der Patienten waren bereit Chemotherapienebenwirkungen für eine Symptomverbesserung oder einen Überlebensgewinn von drei Monaten zu tolerieren. Für einen Überlebensgewinn von 1 Jahr betrug die Akzeptanzrate fast $90 \%$. Überraschenderweise wurde das Überleben der Patienten mit Akzeptanz von Nebenwirkungen kürzer als das der Patienten mit Nichtakzeptanz. Die medianen Überlebenszeiten betrugen 12,5 VS 8 Monate. Schlussfolgerung: Nebenwirkungen einer Chemotherapie werden von einem Großteil der Patienten auch für einen vergleichsweise geringen Überlebensgewinn akzeptiert. Die Akzeptanz von Chemotherapienebenwirkungen ist nicht mit einer verbesserten Prognose vergesellschaftet. Eine positive Beeinflussung der Überlebenszeit durch Motivation und Akzeptanz von Chemotherapienebenwirkungen kann durch diese Untersuchung nicht belegt werden.

\section{PS030}

Treatment with proprotein convertase subtilisin/kexin type 9 antibodies (PCSK9-I) in patients at high cardiovascular risk in clinical routine - new data regarding Familial Hypercholesterolaemia (FH) genotype related safety and effectivity

Friederike Schumann ${ }^{1}$; Tim Hollstein ${ }^{2}$; Elisabeth Steinhagen-Thiessen²; Ursula Kassner $^{2}$

${ }^{1}$ Charité - Universitätsmedizin Berlin, Lipidambulanz - Campus Virchow-Klinikum, Berlin, Germany; ${ }^{2}$ Charité Universitätsmedizin Berlin, Stoffwechselcentrum Lipidclinic, Berlin, Germany

Aim: PCSK9-I reduce low-density lipoprotein cholesterol (LDL-C) and improve cardiovascular risk. The aim of this study is to provide new safety and effectivity data of PCSK9-I with focus on the FH genotype in a "real-world" cohort.

Methods: 781 patients receiving PCSK9-I following recommendations of the German Federal Joint Committee were recruited between 2016 and 2018 at the Outpatient Lipid Clinic of the Charité Berlin. Those patients with high or very high cardiovascular risk according to ESC guidelines did not reach their LDL-C goals under maximal tolerable lipid-lowering therapy or were statin-intolerant. Therefore they were treated with either Alirocumab (75 mg or $150 \mathrm{mg}$ ) or Evolocumab (140 $\mathrm{mg}$ ) every other week. Blood tests were performed at baseline and after one month of PCSKg-I treatment. Patients were asked for side effects 2 weeks after the first injection with a standardized questionnaire. Out of these 781 patients 132 had a genetic test result available. Cases with a positive or likely positive genetic result for $\mathrm{FH}$ (group 1) where combined as well as those with a negative result or one with unknown significance (group 2).

Results: The mean percent reduction of LDL-C after one month was $51.4 \% \pm 17.3$ of patients of group 1 (mean baseline LDL-C $201.3 \mathrm{mg} / \mathrm{dl} \pm$ 76.5) compared to $53.8 \% \pm 15.6$ of patients of group 2 (mean baseline LDL-C was $184.4 \mathrm{mg} / \mathrm{dl} \pm 67.6$ ) and therefore not significantly different $(p=0.426)$. There was no significant difference $(p=0.728)$ in the choice of the PCSKg-I drug or dosage. Side effects were observed in $47.6 \%$ of the patients of group 1 compared to $40 \%$ of those of group 2 ( $p$ $=0.397$ ) but the difference in occurrence of side effects was not significant. There was a significant difference $(p<0.001)$ in both groups regarding the existing lipid-lowering therapy with statins as only $12 \%$ of the patients of group 2 were on high intensity statin therapy compared to $48.8 \%$ of the patients of group 1 . In patients of group 1 without background statin therapy, the LDL-C lowering effect of PCSK9-I was significantly lower $(p=0,001)$ compared to those with high intensity statin therapy $(41 \% \pm 13.3$ vs. $58 \% \pm 17.3)$.

Conclusion: PCSK9-I are safe and effective in a "real-world" cohort. Our study showed no significant difference in safety with respect of the genotype. The LDL-C reduction in both groups was comparable to large clinical trials. In group 1 was a more profound LDL-C lowering effect of PCSKg-I in patients on background statin therapy. We saw the same tendency in group 2 which gives the impression that it's worthwhile to put patients even on a low dose statin therapy to achieve a better efficacy of the PCSK9-I therapy. 


\section{PS031}

\section{Einflussfaktoren und prognostische Wertigkeit der miR-21 Konzentration im Serum von Patienten mit Hepatozellulärem Karzinom (HCC)}

\section{Martin Franck; Kerstin Schütte; Peter Malfertheiner; Alexander Link}

Otto-von-Guericke Universitätsklinikum Magdeburg, Klinik für Gastroenterologie, Hepatologie und Infektiologie, Magdeburg, Germany

Einleitung: Zur Individualisierung von Therapieentscheidungen bei Patienten mit HCC ist eine genaue prognostische Einschätzung notwendig. Hier können in Zukunft neue nicht-invasive Biomarker eine entscheidende Rolle spielen. Die miR-21 ist bei vielen Tumorerkrankungen hochreguliert, so auch im HCC. Dabei geht sie mit einer schlechteren Prognose einher. Zur prognostischen Wertigkeit der miR-21 im Serum gibt es aktuell widersprüchliche Daten und die Validierung in einer europäischen Patientenkohorte fehlt.

Ziele: Wir haben die prognostische Wertigkeit und beeinflussende Faktoren der Serum-miR-21 in einer Kohorte von Patienten mit HCC analysiert. Besonderheiten unserer Studie stellen die europäische Kohorte mit ätiologischer Dominanz des Alkoholabusus sowie einem hohen Anteil an Patienten mit fortgeschrittenen Tumorstadien dar.

Methodik: Im Zeitraum vom 01/2009 bis 04/2011 konnten 91 Patienten mit gesichertem HCC eingeschlossen werden. Die Patienten wurden anhand von klinischen und laborchemischen Daten charakterisiert. Anhand von Kaplan-Meier-Überlebenskurven wurde die prognostische Wertigkeit untersucht. Die Extraktion der gesamten RNA aus dem Serum erfolgte mittels miRNeasy Mini Kit. Die quantitative Analyse der miR-21 erfolgte mittels qPCR. Zur Normalisierung wurde die spiked-in cel-miR-39 verwendet.

Ergebnis: Die Analysen zeigten keine signifikanten Unterschiede im Überleben von Patienten mit hohen und niedrigen Serum-miR-21 Werten $(p=0,2697)$. Weiterhin fanden sich bei den Patienten ähnliche miR-21-Konzentrationen unabhängig vom Child-Pugh-Score $(p=$ $0,7991)$, der BCLC-Klassifikation $(p=0,3947)$ oder der zu Grunde liegenden Ätiologie des HCC $(p=0,6331)$. Die Serum-miR-21 war unabhängig vom Alter $(p=0,2789)$, vom Geschlecht $(p=0,8263)$ und der Vortherapie der Patienten $(p=0,2244)$. Interessanterweise zeigte sich eine signifikant positive Korrelation zwischen Serum-miR-21 und ASAT ( $p$ $=0,0061)$, sowie ein Trend zwischen miR-21 und ALAT $(p=0,1053)$. Es bestand keine Korrelation mit AFP $(p=0,3337)$. Zwischen Serum-miR-21 und Quick konnten wir eine Tendenz zu einer negativen Korrelation nachweisen $(p=0,0666)$. Weiterhin zeigte sich eine signifikant negative Korrelation zwischen miR-21 und Kreatinin $(p=0,0348)$ als Hinweis für eine Beeinflussung der Serum-miR-21 durch die Nierenfunktion.

Zusammenfassung: In dieser europäischen Kohorte von Patienten mit HCC wies die Serum-miR-21 Konzentration keine prognostische Wertigkeit auf. Eine Abhängigkeit von Vortherapie, Ätiologie und Stadium des HCC fand sich nicht. Die Abhängigkeit der Serum-miR-21 Konzentration von Nierenfunktion und Leberschädigung hat eine wichtige translationale Bedeutung, welche in weiteren Studien berücksichtigt werden sollte.
PS032

\section{Epidemiological data from 1515 patients with functional GI diseases under an herbal therapy}

Karen Nieber'; Esther Raskopf ${ }^{2}$; O. Greinert ${ }^{3}$; G Zadoyan ${ }^{3}$; S. Schleicher ${ }^{3}$; Kija Sha-Hosseini ${ }^{3}$; Tankred Wegener ${ }^{4}$; Olaf Kelber ${ }^{5}$; J Singh ${ }^{3}$; R Mösges ${ }^{2}$

'Universität Leipzig, Institut für Pharmazie, Leipzig, Germany; ${ }^{2}$ ClinNovis $\mathrm{GmbH}, \mathrm{R} \& \mathrm{D}$, Köln, Germany; ${ }^{3}$ Universitätsklinikum Köln, Institut für Medizinische Statistik und Bioinformatik, Köln, Germany; ${ }^{4}$ Consulting HMP, R\&D, Weinheim, Germany; ${ }^{5}$ Bayer Consumer Health Division, R\&D, Darmstadt, Germany

Data on patients with functional GI diseases from unselected patient cohorts can improve the understanding of patients' needs in daily practice. Therefore data on patients treated with a herbal medicinal product for this indication, STW 5, were selected from the PhytoVIS study, a non interventional study in 20,870 patients who had used a herbal products within the last 8 weeks before the survey. The aim was to describe the epidemiology, medical needs, and judgement of the perceived usefulness of the therapy expressed by this patient group.

The database contained 24,056 patient questionnaires, which had been documented in physicians' practices and pharmacies by respectively trained students of human medicine or pharmacy who acted as interviewers, in compliance to the ENCePP Code of Conduct [2]. Questionnaires related to STW 5 were evaluated regarding epidemiological data on gender, age and disease state as well as on the use of the medicine and its perception.

Of all datasets, 1515 could be retrieved containing STW5, including patients from pediatric and geriatric age groups most of them being females, with a broad range of functional Gl symptoms. Usefulness was predominantly rated very good to good, also in the patients from pediatric age groups, with 71 patients. Tolerability in all age groups was predominantly very good, in accordance to the published data from more than 50,000 patients treated with this herbal medicine [3].

PhytoVIS turned out to be a suitable tool to study the epidemiology of patients with functional GI diseases, under treatment with the herbal medicinal product STW 5 , showing tolerability, safety and efficacy based on the rating of the patients. Through the high number of users included, special patient groups like children and elderly were also covered. As could be shown on this example, the database is accordingly an excellent source for medical information on patients using herbal medicinal products.

Acknowledgment: The study is supported by Kooperation Phytopharmaka GbR, Bonn, Germany

[1] Raskopf et al. Z Phytother 2017, 38(S 01): S1-S44\#; 2. European Network of Centres for Pharmacoepidemiology and Pharmacovigilance (ENCePP), 2018, EMA/929209/2011; 3. Storr M et al. Pharmakon 2016; 4: 356 - 364 


\section{PS033}

\section{Behandlung funktioneller Magen-Darm Erkrankungen bei Kindern: Ein systematisches Review}

\author{
Johanna Möller'; Olaf Kelber²; Karen Nieber ${ }^{3}$
}

${ }^{1}$ Steigerwald Arzneimittelwerk, Phytomedicines Supply and Development Center, Bayer Consumer Health Division, R\&D, Darmstadt, Germany; ${ }^{2}$ Bayer Consumer Health Division, R\&D, Darmstadt, Germany; ${ }^{3}$ Universität Leipzig, Institut für Pharmazie, Leipzig, Germany

Einleitung: Funktionelle Magen-Darm Erkrankungen, zu denen unter anderem die Funktionelle Dyspepsie (FD) und das Reizdarmsyndrom (IBS, Irritable Bowel Syndrome) gehören, sind gekennzeichnet durch Symptome wie Abdominalschmerzen, Übelkeit, Völlegefühl und Stuhlunregelmäßigkeiten. Diese können in einer stark reduzierten Lebensqualität resultiert (1). In Anbetracht der hohen medizinischen Relevanz dieser Indikationen bei Kindern (2) gilt es zu untersuchen, welche Therapiemöglichkeiten in dieser Altersgruppe vorhanden sind. Um dieser Frage nachzugehen, wurde ein systematisches Review durchgeführt.

Methode: Es wurde eine PubMed-Medline Suche gemäß des PRISMA-Statements nach Studien und Reviews betreffend FD und IBS bei Kindern durchgeführt (Suchanfrage: children AND IBS bzw. children AND FD).

Ergebnisse: Die Suche ergab 79 Ergebnisse zu FD bei Kindern, davon $27 \mathrm{zu}$ pharmakologischen Behandlungsoptionen, von denen 2 pflanzliche Arzneimittel waren. Im Falle von IBS ergaben sich 321 Treffer, davon 90 bezüglich pharmakologischer Therapien, darunter 5 pflanzlichen Ursprungs. Bei diesen pflanzlichen Arzneimitteln handelte es sich um Flohsamen, Pfefferminzöl und STW 5 (Iberogast) sowie Kurkuma, Cannabis, Aloe vera und Ingwer, wobei sich nur bei Flohsamen, Pfefferminzöl und STW 5 die Angaben auf klinische Studien bei Kindern stützten $(3,2,4,5,1)$. Bei FD wurden Sanddorn und STW 5 als pflanzliche Therapieoptionen identifiziert, wobei zu beiden klinische Daten vorliegen (6). Nur STW 5 zeigt für beide Krankheiten belegte Wirksamkeit bei Kindern. Die Suche nach weiteren Daten bezüglich der Evidenz von STW 5 zur Anwendung in dieser Altersgruppe ergab verschiedene retrospektive $(7,8)$ sowie prospektive $(9,10)$ Studien. Diese Anwendungsbeobachtungen beinhalteten insgesamt 44.315 Kinder und zeigten ein gutes Sicherheitsprofil mit nur wenigen leichten Nebenwirkungen in dieser Altersgruppe und eine nachvollziehbare Einschätzung des therapeutischen Nutzens.

Diskussion und Schlussfolgerung: Funktionelle Magen-Darm Erkrankungen sind ein wichtiges Indikationsgebiet mit besonderem Interesse in der Pädiatrie. Pflanzliche Arzneimittel bieten hier nebenwirkungsarme und wirksame Therapieoptionen und nehmen so eine wichtige Rolle in dieser Indikation ein. Bei sehr heterogenen Evidenzgraden der verschiedenen Präparate zeigt STW 5 die überzeugendste Wirksamkeit bei FD und IBS, während Flohsamenschalen und Pfefferminzöl bzw. Sanddorn nur bei IBS bzw. FD nachgewiesene Wirksamkeit bei Kindern aufwiesen.

[1] Malfertheiner, Dig Dis. 2017; 35 Suppl 1; 25-29, 2. Shulman et al., Clin Gastroenterol Hepatol. 2017, 5:712-719, 3. Charrois et al., Pediatr Rev. 2006, 7:49-51, 4. Grigoleit und Grigoleit, Phytomedicine 2005, 8:601-6, 5. Fifi et al., Nutrients 2018, 11 pii: E1715, 6. Hell et al. J Nucl Med. 2013,1:3843, 7. Kelber Z Phytother, 2010, 31:40, 8. Gundermann und Hänicke, Päd. 2004, 10:408, 9. Vinson und Radke, Gatroenterol., 2011, 140:102, 10. Nazarenko et al., Med. novosti 2008, 10:79-83

\section{PS034}

Pharmacoepidemiological research in the paediatric population: Children data from the PhytoVIS study, a NIS in 20,870 users of herbal medicinal products

Esther Raskopf'; Oliver Greinert ${ }^{2}$; Gregor Zadoyan²; Sabine Schleicher ${ }^{2}$; Kija Sha-Hosseini ${ }^{2}$; Tankred Wegener ${ }^{3}$; Olaf Kelber ${ }^{4}$; Karen Nieber ${ }^{5}$; Jaswinder Singh'; Ralph Mösges'

${ }^{1}$ ClinNovis GmbH, I\&D, Köln, Germany; ${ }^{2}$ Universitätsklinikum Köln, Institut für medizinische Statistik und Bioinformatik, Köln, Germany; ${ }^{3}$ Consulting HMP, XY, Weinheim, Germany; ${ }^{4}$ Bayer Consumer Health Division, R\&D, Darmstadt, Germany; ${ }^{5}$ Universität Leipzig, Institut für Pharmazie, Bonn, Germany

Introduction: Pharmacoepidemiological research can be an important source of information on the use of medicinal products in the broad range of patients attending the practice of internists and general practitioners. This applies even more to the pediatric patient group and to herbal medicinal products, where clinical study data are rare. To address this gap, data from the PhytoVIS study, presumably the world's largest pharmacoepidemiological study on the use of herbal medicinal products [1], were evaluated with the view on the pediatric population.

Methods: The PhytoVIS data set contains information on the epidemiology of the patients and the therapeutic indication, the efficacy and tolerability of the medicinal products used by them, which have been captured in doctor's practices and pharmacies in compliance to the ENCePP Code of Conduct [2]. When screening it for pediatric data, information on indications, treatments, co-medication and tolerability was evaluated.

Results: Overall, 2203 data sets from the pediatric population could be evaluated, thereof 297 for patients below 2 years, 505 from patients aged 2-5 years, 578 from age 6-11 years, and 823 from age 12-17 years. Of those, $67.7 \%$ were treated because of common cold and fever, $13.6 \%$ due to digestive complaints, $4.9 \%$ because of skin diseases, $3.5 \%$ due to sleep disturbances and anxiety and $10.3 \%$ because of other complaints. A co-medication was documented in $25.0 \%$ of the patients. The efficacy of the therapy was rated very good in $48.8 \%$ of the patients, good to moderate in $36.3 \%$, modest in $10.7 \%$ and missing in $4.1 \%$. Tolerability was good in $93.6 \%$ and moderate in $5.5 \%$ of patients.

Discussion and conclusion: The data sheds light to a field of pharmacotherapy about which up to now not much was known, and give a picture of the use of herbal medicines in an unselected cohort of pediatric patients. The information gives a better understanding on the way these medicinal products are used in this age group and are also good background information for therapeutic decisions for these patients and their perception. Further age- and indication specific evaluations are desirable.

Acknowledgment: The study is supported by Kooperation Phytopharmaka GbR, Bonn, Germany

\section{[1] Raskopf et al. Z Phytother 2017, 38(S 01): S1-S44}

[2] European Network of Centres for Pharmacoepidemiology and Pharmacovigilance (ENCePP), 2018, EMA/929209/2011 


\section{PS035}

The use of a herbal medicine, STW 42, in the light of the PhytoVIS study, a NIS in 20,870 users of herbal medicinal products

Esther Raskopf'; Oliver Greinert ${ }^{2}$; Gregor Zadoyan ${ }^{2}$; Sabine Schleicher ${ }^{2}$; Kija Sha-Hosseini ${ }^{2}$; Tankred Wegener ${ }^{3}$; Olaf Kelber ${ }^{4}$; Karen Nieber ${ }^{5}$; Jaswinder Singh ${ }^{2}$; Ralph Mösges ${ }^{2}$

${ }^{1}$ ClinNovis GmbH, I\&D, Köln, Germany; ${ }^{2}$ Universitätsklinikum Köln, Institut für Medizinische Statistik und Bioinformatik, Köln, Germany; ${ }^{3}$ Consulting HMP, XY, Weinheim, Germany; ${ }^{4}$ Bayer Consumer Health Division, R\&D, Darmstadt, Germany; ${ }^{5}$ Kooperation Phytopharmaka GbR, XY, Bonn, Germany

Introduction: STW 42, a marshmallow-based cough medicine, is frequently used in the therapy of mucosal irritation with dry cough, a condition frequently occurring in the cold season, and as a merely local mode of action, in contrast to a number of other therapeutic options with more systemic effects and, accordingly, a higher potential of side effects. As a potential source of data on the epidemiology of patients using medicinal products, as well as for documenting their subjective rating of the efficacy and tolerability, the PhytoVIS study was accessed, a pharmacoepidemiological data base on the use of herbal medicinal products [1], containing data from 20,870 users of herbal medicinal products, which have been captured in doctors' practices and pharmacies in compliance to the ENCePP Code of Conduct [2].

The database was screened for users of STW 42 and information on indications, treatments, co-medication and tolerability was evaluated.

Data sets from 141 users of STW 42 syrup or lozenges could be retrieved, of which 43 were children. All patients, rated the treatment predominantly very good or good to moderate, with excellent tolerability.

Together with the data in more than thousand patients documented in earlier surveys, these data underline the usefulness and tolerability of marshmallow products, supporting the use of these medicines not only in adults, but also in the pediatric patients.

Acknowledgment: The study is supported by Kooperation Phytopharmaka GbR, Bonn, Germany

[1] Raskopf et al. Z Phytother 2017, 38(S 01): S1-S44

[2] European Network of Centres for Pharmacoepidemiology and Pharmacovigilance (ENCePP), 2018, EMA/929209/2011

\section{PS036 \\ Herbal medcicinal products in irritable bowel syndrome: Mechanisms of action of STW 5}

Careen Fink'; Olaf Kelber ${ }^{2}$; Sabine Rabini ${ }^{1}$; Ahmed Madisch ${ }^{3}$

${ }^{1}$ Steigerwald Arzneimittelwerk, Phytomedicines Supply and Development Center, Bayer Consumer Health Division, Medical and Clinical affairs Phytomedicines, Darmstadt, Germany; ${ }^{2}$ Bayer Consumer Health Division, R\&D, Darmstadt, Germany; ${ }^{3}$ KRH Klinikum Siloah, Gastroenterology, Interventional Endoscopy, Diabetology, Hannover, Germany

Introduction: Herbal treatment options are increasingly used in functional gastroenterological disorders (FGID) like irritable bowel syndrome (IBS), while an understanding of their mechanisms of action is often lacking. According to recent national and international therapeutic guidelines $(e .9,1,2,3)$, STW 5 , a combination product of nine herbal extracts, is an evidence-based treatment option in IBS. The question was, which mechanisms of action are involved in its therapeutic effect.

Materials and Methods: To warrant completeness, a systematic search according to the PRISMA statement was conducted in order to retrieve all data on STW 5 or its trade name (lberogast), using PubMed, Toxlit and BIOSIS. Identification of data on the mechanisms of action was then done manually. In addition hand searching was done and text books were screened, to get a complete picture.

Results and Discussion: The search identified 468 publications. A considerable number of publications on spasmolytic as well as prokinetic activities could be identified, as well as on prosecretory effects. Also data showing that the product can counteract inflammatory changes as well as an intestinal hypersenstitivity and hyperpermeability were found. Even a beneficial effect on the microbiota was described. Accordingly, the product has a multitude of mechanisms of action.

Conclusions: In IBS, a number of therapeutic options with different mechanisms of action are used. A search for the mechanisms of action of a herbal treatment used in this indication (STW 5 ) revealed not just one, but a multitude of mechanisms of action. This confirms for this product, that its action in IBS can be classified as a multi-target action, and makes its therapeutic efficacy in this indication plausible.

[1] Layer et al. 2011. Z Gastroenterol 49:237-93.

[2] Ivashkin et al. 2017. Ross z gastroenterol gepatol koloproktol 27:76-93

[3] Stanghellini V et al. 2016. Gastroenterology 150:1380-1392;

\section{PS037}

Safety assessment of herbal products: Potential shortcomings

Olaf Kelber'; Karen Nieber ${ }^{2}$; Karin $\mathrm{Kraft}^{3}$

'Bayer Consumer Health Division, R\&D, Darmstadt, Germany; ${ }^{2}$ Universität Leipzig, Institut für Pharmazie, Leipzig, Germany; ${ }^{3}$ Universitätsmedizin Rostock, Zentrum für Innere Medizin, Abteilung Komplementäre Medizin, Rostock, Germany

Introduction: The interest in safety assessments of herbal products is increasing, but not their quality, as limitations of available data are often not sufficiently taken into account. To address the issue, a classification of sources of bias and ways out is aimed to.

Materials and Methods: A systematic data base search for reviews in this field, combined with hand searching in text books, was conducted. Sources of bias were classified according to data types involved.

Results and Discussion: Depending from the data involved, different sources of bias were identified:

- Data on quality: Often the great differences of the composition of herbal products prepared from the same plant are neglected, so leading to flaws when tranferring data.

- Non-clinical data: Common pitfalls are the transfer of data from in vitro studies to the clinical setting, without taking into account the influence of ADME. Often also effects from sublethal high-dose settings are used without sufficiently taking into account dose dependency or, especially e.g. in carcinogenicity studies, methodological ambiguities [1]. 
- Data from clinical studies and post marketing surveillance: Referring to studies conducted with products of a different composition can lead to misleading attribution of efficacy to inefficient products and vice versa, lack of differentiation between negative studies and failed studies can lead to wrong conclusions on inefficacy, the evaluation of safety data is often flawed by neglecting background incidences, as e.g. in case of hepatotoxicity [2] by protopathic bias, and by the awareness and views of authors of case reports [3].

Conclusions: A higher awareness of common pitfalls in the assessment of safety data on herbal products is needed, e.g. in case of hepatotoxic risks, if we want to avoid that methodological artefacts and misperceptions of the generalizability of data continue to influence our view of the safety of herbal products, both by neglecting risks, as, more abundant, by exaggerating non-existing risks.

[1] IARC 2016, IARC monographs, vol.108;

[2] Andrade et al., Pharmacogenomics. 2009 10(9):1467-87

[3] Horwitz R.I. and Feinstein A.R., Am J Med. 1980, 68(2):255-8

\section{PS038 \\ Herbal medicines containing alcohol in children: Pharmaepidemiological data}

Karen Nieber'; Olaf Kelber; Johanna Möller; S. Verjee ${ }^{4}$; Eva Gorgus ${ }^{4}$; Dieter Schrenk ${ }^{4}$

${ }^{1}$ Universität Leipzig, Institut für Pharmazie, Leipzig, Germany; ${ }^{2}$ Steigerwald Arzneimittelwerk GmbH, Phytomedicines Supply and Development Center, Bayer Consumer Health Division, R\&D, Darmstadt, Germany; ${ }^{3}$ Steigerwald Arzneimittelwerk, Phytomedicines Supply and Development Center, Bayer Consumer Health Division, R\&D, Darmstadt, Ghana; ${ }^{4}$ Universität Kaiserslautern, Lebensmittelchemie und Toxikologie, Kaiserslautern, Germany

Einleitung: Zu den häufigsten Indikationen in der internistischen Praxis gehören funktionelle gastrointestinale Erkrankungen und Erkältungskrankheiten, gerade auch bei Kindern, und hier werden häufig flüssige Darreichungsformen eingesetzt, bei denen die Dosierung gut an unterschiedliche Altersgruppen angepasst werden kann. Obwohl die Produkte im allgemein als sicher gelten, hat der Alkoholgehalt einiger pflanzlicher Arzneimittel gelegentlich Fragen zur Sicherheit gerade bei Kindern ausgelöst. Ziel dieser Studie war es, zu analysieren, in welchem Umfang Kindern bei Anwendung dieser Arzneimittel Alkohol aufnehmen, und wie dies in Relation zur Aufnahme mit üblichen Lebensmitteln steht.

Methoden: Daten aus Studien mit pflanzlichen Kinderarzneimitteln wurden hinsichtlich der aufgetretenen Nebenwirkungen ausgewertet. Basierend auf diesen Erkenntnissen wurde die Exposition für ein 6jähriges Kind ( $20 \mathrm{~kg} \mathrm{KG}$ ) ermittelt, um den relativen Beitrag zur gesamten Alkohol-Exposition in dieser Altersgruppe abzuschätzen. Diese wurde durch analytische Bestimmung des Alkoholgehaltes in üblichen Lebensmitteln ermittelt.

Ergebnisse: Es wurden 17 nicht-interventionelle Studien mit 10 pflanzlichen Arzneimitteln analysiert, die Alkohol in Dosen von 0,03 bis 0,23 g /Einzeldosis enthielten. Sie umfassten 50.316 Kinder von 0-12 Jahren. Mit üblichen pflanzlichen Arzneimitteln werden, sich wie am Beispiel eines 6jährigen Kindes zeigen ließ, zwischen 70 und $180 \mathrm{mg}$ Alkohol pro Einzeldosis aufgenommen, was bei 3mal täglicher Dosierung $210-540 \mathrm{mg}$ entspricht. Bei einem Körpergewicht von $20 \mathrm{~kg}$ sind dies $10.1-27.0 \mathrm{mg} / \mathrm{kg}$ [1]. In den Studien und bei Spontanmeldungen von mehr als 3 Mio. Kindern ergaben sich keine Hinweise auf alkohol- bedingte Nebenwirkungen [2]. Die Messung des Alkoholgehaltes von bei Kindern üblichen Lebensmitteln mittels Gaschromatographie ergab, dass Orangen-, Apfel- und Traubensaft bis zu 0,77 g / I enthalten, bestimmte Backwaren wie Burgerbrötchen oder Süßmilchbrötchen mehr als 1,2 g Ethanol / $100 \mathrm{~g}$ [3]. Eine aus diesen Daten abgeleitete Extrapolation für die mittlere tägliche Alkoholaufnahme bei einem 6jährigen Kind ergab eine Alkoholexposition von bis zu $23.3 \mathrm{mg} /$ $\mathrm{kg}$, was in derselben Größenordnung wie die Alkoholaufnahme mit pflanzlichen Arzneimitteln liegt.

Schlussfolgerungen: Zusammenfassend legen unsere Daten nahe, dass eine Vielzahl von Nahrungsmitteln, die von Vorschulkindern konsumiert werden, nennenswerte Mengen Alkohol enthalten. Die Aufnahme von Alkohol mit pflanzlichen Arzneimitteln liegt in einer Größenordnung, die mit der täglichen Exposition mit üblichen Lebensmitteln vergleichbar ist, und ist daher nicht mit relevanten Risiken verbunden.

[1] Kelber O et al. 2008, Pharmlnd; 70, 1124-1127; 2. Kelber O et al. 2016, Wien Med Wochenschr, 167:183-188; 3. Gorgus E et al. 2016, J Analyt Toxicol, doi:10.1093/jat/bkwo46

\section{PS039}

Löslicher IL2-Receptor als möglicher Prädiktor für ein Therapieansprechen auf Ustekinumab bei Patienten mit einem chronisch aktivem M. Crohn und TNF induzierter Psoriasis.

Sebastian Beck'; Markus Porthun ${ }^{2}$; Tanja Kuehbacher ${ }^{2}$

'Christian-Albrechts-Universität zu Kiel, Gastroenterologie, Kiel, Germany; ${ }^{2}$ Asklepios Westklinikum Hamburg, Gastroenterologie, Hamburg, Germany

Hintergund und Ziel: Der lösliche Serum IL2-receptor (sIL2-R) ist ein Entzündungsmarker für die immunologische Aktivierung von T-Lymphocyten in der Lamina propria von Patienten mit chronisch entzündlichen Erkrankungen. Es sollte untersucht werden, ob sich der lösliche sIL2-R als möglicher Prädiktor für eine Therapieansprechen von Ustekinumab bei Patienten mit M. Crohn und einer TNF induzierten Psoriasis eignet.

Methoden: 19 Patienten mit chronisch aktivem, therapierefraktärem M. Crohn und einer TNF induzierten Psoriasis (MC) (CDAI > 220), die nicht mehr auf eine Therapie mit TNF Antagonisten ansprachen oder eine Intoleranz entwickelten, wurden mit Ustekinumab behandelt. Vor Therapiebeginn wurde der lösliche Serum IL2-Rezeptor (sIL2-R) im Serum bestimmt. CDAl und CRP wurden vor Therapiebeginn und nach 12 Wochen im Rahmen der Routineuntersuchungen bestimmt.

Ergebnisse: Das mittlere Alter der Patienten war 39 Jahre. 13 Patienten (68.4\%) sprachen auf die Therapie in der Woche 12 an (Reduktion des CDAl um 70 Punkte). 10 der 13 Patienten waren in klinischer Remission (CDAl < 150, 15.8\%). 6 Patienten (31.6\%) sprachen auf die Therapie mit Ustekinumab nicht an. Bei den Responder Patienten war das sIL2-R erhöht oder im höheren Level des Normalbereichs mit einem Mittelwert von 1004.5 U/ml (Normwert 223-710 U/ml) im Gegensatz zu den Nicht-Responder Patienten, bei denen ein Mittelwert von $388.3 \mathrm{U} /$ $\mathrm{ml}$ gemessen werden konnte. Die Differenz der Serumkonzentration vom sll2-R der Responder zu der Non-Responder Patientenkohorte war statistisch signifikant (Mann-Whitney $\mathrm{U}$ test, $\mathrm{P}<0.001$, two-tailed test).

Das mittlere CRP in der Responder Patientenkohorte war $20.1 \mathrm{mg} / \mathrm{l}$ vor Therapiestart und $9 \mathrm{mg} / \mathrm{l}$ in der Woche 12. Die Non-Responder Patientenkohorte wies ein CRP von $9.8 \mathrm{mg} / \mathrm{l}$ vor Therapiestart und $17.4 \mathrm{mg} / \mathrm{l}$ in der Woche 12 auf. Der mittlere CDAl vor Therapiestart in der Responderkohorte war 354, in der Non-Responderkohorte 312. Der CDAI in der Woche 12 in der Responderkohorte war 119, in der Non-Respon- 
derkohorte 312. Die Therapie wurde gut vertragen. Keine spezifischen Nebenwirkungen konnten nachgewiesen werden.

Zusammenfassung: Erhöhter sIL2-R korrelierte signifikant mit dem klinischen Ansprechen auf Ustekinumab bei Patienten mit chronisch aktivem M.Crohn mit einer Intoleranz oder sekundärem Therapieversagen auf TNF Antikörper und einer TNF induzierten Psoriasis. Prospektive, kontrollierte Studien mit großen Patientenkohorten sollten das Potential von löslichem Serum IL2-R als möglichen Prädiktor für ein Therapieansprechen von Ustekinumab bei M.Crohn Patienten untersuchen. Sollten die Ergebnisse dieser kleinen Pilotstudie bestätigt werden, wäre der sIL2-R ein kostengünstiger und leicht zu erhebender Prädiktor für die Therapieentscheidung einer Ustekinumab-Behandlung.

\section{Multimorbidität und Polypharmazie}

\section{PS040 \\ Arzneimittelinteraktionen bei der Therapie der Hepatitis C Virusinfektion - weiterhin relevant für die klinische Praxis?}

Benjamin Schulte'; Maximilian Wübbolding ${ }^{1}$; Kerstin Port'; David Back²; Fiona Marra'; Michael P. Manns'; Markus Cornberg'; Benjamin Maasoumy'; Christoph Höner zu Siederdissen ${ }^{1}$

'Medizinische Hochschule Hannover, Gastroenterologie-Hepatologie-Endokrinologie, Hannover, Germany; ${ }^{2}$ University of Liverpool, Molecular and Clinical Pharmacology, Liverpool, United Kingdom

Einleitung: Bei der Therapie der chronischen Hepatitis C Virusinfektion (HCV) hat sich durch die Zulassung der direkt antiviral wirksamen Substanzen (DAA) das Management von Arzneimittelinteraktionen zu einer neuen klinischen Herausforderung entwickelt. Diese Studie hat zum Ziel i) eine mögliche Veränderung der Häufigkeit von Interaktionen über die Zeit zu analysieren, ii) Patientengruppen mit einem erhöhten Risiko für Interaktionen zu identifizieren und iii) diejenigen Wirkstoffe zu finden, welche am häufigsten an Interkationen beteiligt sind.

Methode: Alle HCV-Patienten, die im Zeitraum von Januar 2014 bis Juli 2018 in der Medizinischen Hochschule Hannover mit DAA behandelt wurden, wurden für die Studie berücksichtigt. Sämtliche Begleitmedikation wurde erfasst und auf Interaktionen mit dem verschriebenen DAA-Regime überprüft. Folgende Klassifizierung wurde vorgenommen: 1) keine Interaktion erwartet, 2) mögliche schwache Interaktion, 3) mögliche signifikante Interaktion und 4) Kombination vermeiden. Zur Bewertung wurde das etablierte Webtool hep-druginteractions. org und im Falle von fehlenden Informationen die Expertenmeinung eines Apothekers herangezogen. Um eine mögliche Veränderung der Häufigkeiten darstellen zu können, wurden folgende Zeiträume definiert: A) Januar 2014 - November 2014, B) November 2014 - August 2016 und C) August 2016 - Juli 2018, basierend auf der Zulassung neuer antiviraler Therapieoptionen.

Ergebnisse: Insgesamt konnten 670 Patienten ausgewertet werden. Das mittlere Alter war 55,5 Jahre (18-85 Jahre; 6,6\% $\geq 75$ Jahre), 44,9\% waren weiblich und $42,2 \%$ hatten eine Leberzirrhose. 350 verschiedene Wirkstoffe kamen in der Begleitmedikation zum Einsatz. Im Median nahm ein Patient drei verschiedene Wirkstoffe ein (o-19 Wirkstoffe). Über die Zeit blieb die Häufigkeit der Patienten ohne Arzneimittelinteraktion konstant (A: 63,0\%, B: 51,2\%, C: 61,5\%). Die Häufigkeit der Patienten mit Kontraindikation (Kategorie 4 ) hingegen verdoppelte sich zwischen Zeitraum A) und B) und blieb ähnlich hoch in Zeitraum C) (A: 2,5\%, B: 5,1\%, C: 4,0\%). Patienten mit Zirrhose (keine Zirrhose: $28,4 \%$,
Zirrhose: 42,4\%) und Patienten mit hohem Alter (18-64 Jahre: 29,0\%, 65-74 Jahre: 56,6\%, $\geq 75$ Jahre: 59,1\%) waren signifikant häufiger von Interkationen der Kategorie 3 betroffen. Interaktionen der Kategorie 3 und 4 bei den aktuellen Regimen Sofosbuvir/Velpatasvir, Glecaprevir/ Pibrentasvir und Elbasvir/Grazoprevir traten oft mit Protonenpumpeninhibitoren, Metamizol, Statinen und Carvedilol auf.

Zusammenfassung: Arzneimittelinteraktionen sind weiterhin relevant für die klinische Praxis und betreffen etwa $40 \%$ der Patienten in dieser großen, deutschen Kohorte. Zirrhose und höheres Alter scheinen Risikofaktoren für das verstärkte Auftreten dieser zu sein. Wirkstoffe mit aktuell erhöhtem Interaktionspotential bei der HCV-Therapie sind Protonenpumpeninhibitoren, Metamizol, Statine und Carvedilol.

\section{PS041 \\ What doctors can do against drug overuse in polypharmacy: a systematic review of clinical trials}

Thomas Gamstätter'; Simone Jacobss'; Claudia Zimmer² ; Daniel Grandt ${ }^{2}$

'Deutsche Gesellschaft für Innere Medizin e.V., Geschäftsstelle, Wiesbaden, Germany; ${ }^{2}$ Klinikum Saarbrücken gGmbH, Klinik für Innere Medizin I, Saarbrücken, Germany;

Background: Multimorbid patients with polypharmacy are at risk for inappropriate prescribing and harmful medication overuse. A recently updated Cochrane review investigating complex and multi-faceted interventions to improve the appropriateness of polypharmacy in the elderly found the evidence for these interventions low to very low and inconclusive.1 There is a lack of evidence summaries on more simplified and pragmatic interventions to manage polypharmacy in multimorbid patients.

Objective: To identify from clinical trials interventions practicing physicians can employ to reduce drug overuse and inappropriate prescribing in polypharmacy and achieve meaningful endpoints in multimorbid patients.

Methods: A sensitive search protocol of original research articles indexed in MEDLINE from 2014 to 2018, including related primary sources. Reporting followed the PRISMA guidelines. We excluded non-interventional studies, trial protocols, feasibility studies, and trials in pediatric populations. We further excluded multi-professional interventions and studies addressing the roles of nurses and pharmacists. The authors screened articles, assessed risk of bias, and extracted data according to standardized conventions.

Results: A total of 4323 citations were screened. 2892 studies were deemed irrelevant to the research subject, 1431 were assessed for eligibility. 43 studies met the inclusion criteria and were further assessed for methodologic quality, relevance and clinical applicability.

Select findings favoring the intervention included the use of the START/STOPP criteria and the FORTA list addressing drug over- and under-treatment in older hospitalized patients. Select findings with no effect on the primary outcome included a protocol for reduction of fall-risk-increasing drugs (FRID) in older fallers and an intervention targeting the number of medications and health-related quality of life by an intensification of the doctor-patient dialogue about treatment preferences and patient priorities.

The quality of evidence was very low due to imprecision, indirectness and serious risk of bias, occurring predominantly in the domains of selective outcome reporting, allocation concealment, and blinding 
which did not allow for valid effect estimates for prespecified critical outcomes.

Discussion: Despite the growing challenges of care for patients with polypharmacy and multimorbidity, evidence from clinical trials that address critical outcomes is limited. It is unclear whether the identified interventions that can be employed by individual physicians to reduce drug overuse and inappropriate prescribing in multimorbid patients, such as protocols for medication review, resulted in clinical improvements.

[1] Rankin A, Cadogan CA, Patterson SM, Kerse N, Cardwell CR, Bradley $M C$, Ryan $C$, Hughes $C$. Interventions to improve the appropriate use of polypharmacy for older people. Cochrane Database of Systematic Reviews 2018, Issue 9. Art. No.: CDoo8165. DOI: 10.1002/14651858. CDoo8165.pub4.

\section{PS042 \\ Is lower handgrip strength associated with increased arterial stiffness?}

Maximilian König'; Fabiola Lugano²; Ilja Demuth²; Elisabeth Steinhagen-Thiessen ${ }^{2}$

'Charité - Universitätsmedizin Berlin, Klinik für Nephrologie und internistische Intensivmedizin, Berlin, Germany; ${ }^{2}$ Charité - Universitätsmedizin Berlin, Medizinische Klinik für Endokrinologie AB Lipidstoffwechsel, AG Biologie des Alterns, Berlin, Germany;

Background: It has been shown that handgrip strength is a strong predictor of cardiovascular and non-cardiovascular mortality and a moderately strong predictor of incident cardiovascular disease. Moreover, low grip strength was associated with higher case-fatality rates both as to cardiovascular or non-cardiovascular disease. It has been suggested that unmeasured factors, such as endothelial dysfunction as well as arterial stiffness might mediate the association between muscle strength and cardiovascular events.

We have measured pulse wave velocity (PWV) and handgrip strength (HGS), along with potential confounders both in the LipidCardio study, which is a cohort of 1,005 consecutive patients, recruited on the occasion of elective coronary angiography at the Charité-Universitätsmedizin Berlin during 2016-2018, and in the Berlin Aging Study 2 (BASE-II). In this analysis of merged baseline data from both studies we aimed to investigate if there is an independent association of handgrip strength and arterial stiffness, as assessed by pulse wave velocity (PWV).

Methods: We calculated the maximum handgrip strength, using al measurements on both arms available. PWV in $\mathrm{m} / \mathrm{s}$ was measured with the Mobil-O-Graph ${ }^{\circledast}$ device according to manufacturer's instructions.

Results: Measurements of both HGS and PWV were available from 598 (59.5\%) participants of the LipidCardio cohort and 915 (41.7\%) participants of the BASE-II cohort, respectively. The median age of the combined dataset ( $n=1513$ ) was 68.6 years (range 22.0 to 91.2 years). $63.1 \%$ of the participants were male. In the combined dataset $34.4 \%$ had coronary artery disease (CAD).

There was evidence $(p<0.001)$ of a moderate negative correlation of HGS and PWV both in males $(r=-0.405)$ and females $(r=-0.499)$. Unadjusted PWV decreased by about $1 \mathrm{~m} / \mathrm{s}$ per increase in tertile of hand grip strength.
In multivariable regression analysis, stratified for sex, and adjusted for age, CAD, BMI, systolic blood pressure and current smoking there was reasonable evidence in support of an association between HGS and PWV in both men and women, respectively. There was no evidence of important effect modification by sex, and the association was evident in both original cohorts, LipidCardio and BASE-II. On average, per tertile decrease in HGS PWV increased by $0.12 \mathrm{~m} / \mathrm{s}$ (95\% confidence interval 0.08 to $0.18 \mathrm{~m} / \mathrm{s}, \mathrm{p}<0.001$ ). Similarly, odds for low handgrip strength (lowest tertile) were increased by 4.1 times (95\% confidence interval 2.9 to 5.7) comparing participants with PWV $>11$ vs. $P W V \leq 11 \mathrm{~m} / \mathrm{s}$.

Conclusion: There was reasonable evidence for an independent association of (low) HGS with (elevated) PWV. Increased arterial stiffness might be a factor contributing to and explaining part of the increased cardiovascular risk observed in individuals with lower handgrip strength.

\section{Interdisziplinarität in der Inneren Medizin}

\section{PS043 \\ Effects of arteriovenous fistula on blood pressure in patients with end-stage renal disease: A systematic meta-analysis}

Sean S. Scholz'; Davor Vukadinovic'; Lucas Lauder'; Sebastian Ewen'; Christian Ukena ${ }^{2}$; Raymond R. Townsend ${ }^{3}$; Stefan Wagenpfeil ${ }^{4}$; Michael Böhm'; Felix Mahfoud'

'Saarland University Hospital, Klinik für Innere Medizin III, Kardiologie, Angiologie und Internistische Intensivmedizin, Homburg, Germany; ${ }^{2}$ Universitätsklinikum des Saarlandes, Klinik für Innere Medizin III, Kardiologie, Angiologie und Internistische Intensivmedizin, Homburg, Germany; ${ }^{3}$ University of Pennsylvania Medical Center, Perelman School of Medicine, Philadelphia, United States; ${ }^{4}$ Saarland University, Campus Homburg/ Saar, Institut für Medizinische Biometrie, Epidemiologie und Medizinische Informatik, Homburg, Germany

Objective: Central arteriovenous fistula (AVF) creation is under investigation for treatment of severe hypertension. We evaluated the effects of AVF for initiation of hemodialysis (HD) on systolic (SBP), diastolic (DBP), and mean arterial blood pressure (MAP) in patients with end-stage renal disease (ESRD).

Methods: Data search included PubMed, Web of Science, and the Cochrane Library. A systematic review and meta-analysis of peer-reviewed studies with $\geq 10$ participants, reporting the effects of the creation/ligation of an AVF on BP in patients with ESRD was performed according to the PRISMA, PRISMA-P, and Risk of Bias in Non-Randomized Studies (ROBINS-I) criteria by the Cochrane Bias Methods Group.

All studies in which the results could have been biased by HD were excluded.

Results: A total of 13 trials including 388 patients with ESRD (AVF creation, $n=161$; AVF ligation, $n=227$ ) fulfilled the criteria and were subsequently analyzed. Average BP in patients with no/closed AVF was $139.9 / 77.6 \mathrm{~mm} \mathrm{Hg}$ with a mean MAP of $96.1 \mathrm{~mm} \mathrm{Hg}$. Following creation of AVF, SBP significantly decreased by $10.5 \mathrm{~mm} \mathrm{Hg}(\mathrm{p}<0.001)$, DBP by $5.9 \mathrm{~mm} \mathrm{Hg}(p<0.001)$, and MAP by $6.6 \mathrm{~mm} \mathrm{Hg}(p=0.02)$, whereas after ligation SBP tend to increase by $5.2 \mathrm{~mm} \mathrm{Hg}(p=0.07)$, DBP by $3.8 \mathrm{~mm}$ $\mathrm{Hg}(p=0.02)$, and MAP by $3.7 \mathrm{~mm} \mathrm{Hg}(p=0.07)$ during short- to longterm follow-up.

Conclusion: Creation of AVF significantly decreases BP in patients with ESRD, whereas BP tend to increase after ligation. These findings 
illustrate the hemodynamic consequences of AVF which is under investigation for severe hypertension.

\section{PS044 \\ Underdetection of left heart failure in COPD: Results from the COSYCONET cohort}

Peter Alter'; Barbara A. Mayerhofer ${ }^{2}$; Kathrin Kahnert'; Henrik Watz; Benjamin Waschki ${ }^{4}$; Frank Biertz ${ }^{5}$; Robert Bals ${ }^{6}$; Claus F. Vogelmeier'; Rudolf A. Jörres²

'Philipps-Universität Marburg, Innere Medizin, Pneumologie und Intensivmedizin, Marburg, Germany; ${ }^{2}$ Ludwig-Maximilians-Universität München, Institut und Poliklinik für Arbeits-, Sozial- und Umweltmedizin, München, Germany; ${ }^{7}$ Ludwig-Maximilians-Universität München, Medizinische Klinik und Poliklinik V, München, Germany; ${ }^{3}$ LungenClinic Grosshansdorf, Pneumologisches Forschungsinstitut, Grosshansdorf, Germany; ${ }^{4}$ Pneumologisches Forschungsinstitut an der LungenClinic Grosshansdorf GmbH und Universitäres Herzzentrum Hamburg $\mathrm{GmbH}$, Allgemeine und interventionelle Kardiologie, Grosshansdorf und Hamburg, Germany; ${ }^{5}$ Medizinische Hochschule Hannover, Institut für Biometrie, Hannover, Germany; ${ }^{6}$ Universitätsklinikum des Saarlandes, Innere Medizin V - Pneumologie, Allergologie und Intensivmedizin, Homburg, Germany;

Rationale: Cardiovascular comorbidities are frequent in COPD and worsen the prognosis. Due to the overlap of symptoms, in particular dyspnea, the detection of concomitant left heart failure remains a challenge in COPD, but is required for adequate treatment. For therapy of systolic left heart failure, diuretics, beta-blockers and renin-angiotensin-aldosterone (RAAS) inhibitors are recommended. Neither the frequency of left heart failure in COPD, nor the frequency of sufficient treatment is well known. To elucidate this question, we evaluated echocardiographic criteria and heart failure medication in a large cohort of patients with stable COPD.

Methods: From the baseline data of the German COSYCONET cohort those of patients with full and plausible lung function and echocardiographic data including left ventricular ejection fraction (LVEF) and end-diastolic diameter (LVEDD) were analyzed. Systolic left heart failure was assumed for LVEF $\leq 50 \%$ or LVEDD $>56 \mathrm{~mm}$. Medication was assessed via a detailed questionnaire.

Results: Overall, 1591 patients fulfilled the inclusion criteria (mean \pm SD age: $64.4 \pm 8.6$ years; GOLD o-4: $n=230 / 126 / 614 / 498 / 123$ ). Presence of arterial hypertension was reported in $54.1 \%$. Systolic heart failure according to echocardiography was detected in 239 patients (15.0\%). The magnitude of airway obstruction ( $F E V_{1}, 55$ vs $58 \%$ pred, $\mathrm{p}=0.039$ ) was slightly more severe in the heart failure subgroup, whereas no difference was found for lung hyperinflation (ITGV, 141 vs 145\%pred, $\mathrm{p}=0.148$ ) when compared with the remaining cohort. Of note, the rate of diuretics use (including aldosterone antagonists) was not different between the groups ( $21.8 \mathrm{vs} 17.2 \%, \mathrm{p}=0.099$ ), whereas a slightly increased rate of RAAS inhibitors ( 50.2 vs $42.0 \%, p=0.029$ ), but a markedly increased rate of beta-blockers ( 31.8 vs $19.2 \%, p<0.001$ ) was found in the heart failure group compared to the remaining cohort.

Conclusion: Contrary to expectation, the overall rate of heart failure treatment in terms of diuretics was not significantly elevated in patients identified via LVEF reduction or LV dilatation. Although the prescription rate of beta-blockers (and marginally that of RAAS inhibitors) was higher in this subgroup, the total frequency remained low, in particular when taking into account other indications for these drugs, such as arterial hypertension with a reported prevalence of $>$ $50 \%$. Assuming the adequacy of echocardiographic criteria, these observations raise the suspicion that the presence of left heart failure is undetected and untreated in a significant proportion of patients with COPD, which bears the risk of worsening their prognosis.

\section{PS045 \\ Association of pulse pressure and double product with cardiovascular and overall mortality in LURIC}

Babak Yazdani; Marcus Kleber; Yücel Gökhan; Winfried März; Bernhard Krämer

Universitätsmedizin Mannheim, V. Medizinische Klinik für Nephrologie, Hypertensiologie, Endokrinologie, Diabetologie, Rheumatologie, Mannheim, Germany

Background: Systolic (SBP) and diastolic blood pressure (DBP) as well as mean arterial pressure (MAP) are already known as important predictors respectively risk factors for cardiovascular mortality. Pulse pressure (PP) is considered as an easily available marker of vascular stiffness and the double product (DP; SBP $x$ heart rate (HR)) as a marker of cardiac workload. Therefore we extended our analysis of outcome parameters by use of PP and DP.

Methods: We retrospectively analysed data from the Ludwigshafen Risk and Cardiovascular Health (LURIC) study.

Results: Long-term data from 3316 patients undergoing coronary angiography showed that by increasing SBP by $1 \mathrm{mmHg}$ the risk of both cardiovascular and overall mortality rose by $0.9 \%$. However, there was no significant relationship between DBP and mortality. A higher PP of $1 \mathrm{mmHg}$ resulted in a higher cardiovascular mortality risk of $1.6 \%$ and an overall mortality risk of $1.7 \%$. Increasing DP by 1 standard deviation was associated with a $26.1 \%$ higher risk of cardiovascular mortality and $25.7 \%$ higher risk of overall mortality.

Conclusions: We provide evidence that not only the classic standard blood pressure parameters SPB and MAP predict cardiovascular mortality, but also that PP and DP are powerful predictors of cardiovascular mortality risk in a cardiovascular risk population.

\section{PS046}

Modifizierte Immunzellen inhibieren die murine Lupusnephritis und induzieren in-vivo regulatorische Zellpopulationen

Claudius Speer'; Daniela Kim'; Christian Kleist'; Christian Morath²; Anita Schmitt ${ }^{3}$; Michael Schmitt ${ }^{4}$; Claudia Sommerer ${ }^{1}$; Andrea Steinborn ${ }^{5}$; Florian Kälble; ; Christian Nusshag'; Lei Wang ${ }^{2}$; Alexander Kunz ${ }^{2}$; Hanns-Martin Lorenz'; Martin Zeier'; Matthias Schaier ${ }^{2}$

'Universität Heidelberg, Nephrologie, Heidelberg, Germany; ${ }^{2}$ Universität Heidelberg / TolerogenixX GmbH, Nephrologie, Heidelberg, Germany; ${ }^{3}$ Universität Heidelberg / Tolerogenix X GmbH, Hämatologie und Rheumatologie, Heidelberg, Germany; ${ }^{4}$ Universität Heidelberg, Hämatologie und Rheumatologie, Heidelberg, Germany; ${ }^{5}$ Universität Heidelberg, Gynäkologie und Geburtshilfe, Heidelberg, Germany

Hintergrund: Modifizierte Immunzellen (MIC) sind mononukleäre Zellen, die nach Inkubation mit Mitomycin C immunsuppressive Eigenschaften aufweisen(1). Wir konnten zeigen, dass eine syngene MIC Zelltherapie die experimentelle Autoimmunencephalitis inhibiert und in verschiedenen Transplantationsmodellen eine Spender-spezifische Hyporesponsivität erzeugt(2-4). Ziel dieser Studie war es, unsere Erkenntnisse auf das Krankheitsbild des SLE zu übertragen.

Methoden: Splenozyten syngener NZB/W F1 Mäuse wurden aus einem Spendertier isoliert, mit Mitomycin $C$ inkubiert und in die 
Schwanzvene injiziert. Gruppe 1 erhielt keine Zelltherapie, Gruppe 2 eine einmalige Standarddosis mit $1.5 \times 10 \mathrm{~h} 8 / \mathrm{kg}$ KG MIC Zellen und Gruppe 3 eine repetitive MIC Zelltherapie mit 1.5x10h8/kg KG MIC Zellen an Woche 1, 2 und 3. Gruppe 4 erhielt eine repetitive Zelltherapie vor Krankheitsbeginn als präemptiver Therapieansatz. Die Krankheitsaktivität wurde hinsichtlich Gewichtsverlust, Proteinurie und Serumkreatinin bestimmt. Die Endpunkte waren Tag 40 nach Therapiebeginn, eine Proteinurie $\geq 3 \mathrm{~g} / \mathrm{l}$ an 2 aufeinander folgenden Wochen oder ein Gewichtsverlust von $>30 \%$ des Körpergewichts. Um die Ausprägung der Lupusnephritis zu beurteilen wurden histopathologische PAS und HE Färbungen der Nieren durchgeführt. Zusätzlich wurden regulatorische Zellpopulationen gemessen.

Ergebnisse: Durch eine MIC Zelltherapie konnte die Progression der murinen Lupusnephritis bei BMF1 Mäusen verhindert werden. Die Proteinurie und das Serumkreatinin waren sowohl in Gruppe 1 als auch in Gruppe 4 signifikant niedriger im Vergleich zur Kontrollgruppe. Gruppe 3 war hinsichtlich der Proteinurie und des Serumkreatinins der Kontrollgruppe nicht signifikant überlegen. Der kombinierte Endpunkt wurde von Gruppe 1 (67\%) im Vergleich zu Gruppe 2 (14\%), Gruppe 3 (14\%) und Gruppe 4 (0\%) signifikant häufiger erreicht. Histologisch zeigte sich bei den verschiedenen Therapiegruppen im Vergleich zur Kontrollgruppe eine erhaltene renale Struktur. Zusätzlich kam es bei Tieren nach MIC Zelltherapie zu einer Induktion von regulatorischen CD8+ T-Zellen und regulatorischen B-Zellen, während doppelt-negative T-Zellen signifikant erniedrigt waren.

Diskussion: Eine MIC Zelltherapie in Standarddosierung inhibiert die Progression der aktiven Lupusnephritis. Als präemptiver Therapieansatz konnte darüber hinaus der Krankheitsbeginn der murinen Lupusnephritis verhindert werden. Wir konnten bereits im präklinischen EAE Modell und auch in einer ersten klinischen Phase-I Studie an 10 nierentransplantierten Patienten zeigen, dass eine MIC Zelltherapie eine Induktion regulatorischer Zellpopulationen einleitet(2-4). Übereinstimmend konnten wir nach MIC Zelltherapie einen erhöhten Anteil an CD8+ Tregs und CD5+CD1h Bregs delektieren. Die MIC Zelltherapie stellt einen spannenden, klinisch anwendbaren Therapieansatz dar, der bereits am Menschen angewendet werden konnte, und sich als effektive in der Inhibierung der murinen Lupusnephritis erwies.

[1] Mitomycin C-treated dendritic cells inactivate autoreactive T cells: toward the development of a tolerogenic vaccine in autoimmune diseases. Terness P, Oelert T, Ehser S, Chuang JJ, Lahdou I, Kleist C, Velten F, Hämmerling GJ, Arnold B, Opelz G. Proc Natl Acad Sci U S A. 2008 Nov 25;105(47):18442-7. doi: 10.1073/pnas.0807185105. Epub 2008 Nov 18.

[2] Mitomycin C-treated antigen-presenting cells as a tool for control of allograft rejection and autoimmunity: from bench to bedside. Terness P, Kleist C, Simon H, Sandra-Petrescu F, Ehser S, Chuang JJ, Mohr E, Jiga L, Greil J, Opelz G.

[3] Mitomycin-C-treated peripheral blood mononuclear cells (PBMCs) prolong allograft survival in composite tissue allotransplantation. Radu CA, Kiefer J, Horn D, Kleist C, Dittmar L, Sandra F, Rebel M, Ryssel H, Koellensperger E, Gebhard MM, Lehnhardt M, Germann G, Terness P. J Surg Res. 2012 Aug;176(2):e95-e101. doi: 10.1016/j.jss.2011.12.032. Epub 2012 Mar 10.

[4] Immunosuppressive properties of mitomycin C-incubated human myeloid blood cells (MIC) in vitro. Dittmar L, Mohr E, Kleist C, Ehser S, Demirdizen H, Sandra-Petrescu F, Hundemer M, Opelz G, Terness P. Hum Immunol. 2015 Jul;76(7):480-7. doi: 10.1016/j.humimm.2015.06.008. Epub 2015 Jun 11.

\section{PS047}

Na-channel NaV1.8 as a new selective target for treating atrial arrhythmogenesis

Steffen Pabel'; Shakil Ahmad ${ }^{2}$; Petros Tirilomis ${ }^{2}$; Thea Stehle ${ }^{2}$; Nataliya Dybkova $^{2}$; Philipp Bengel ${ }^{2}$; Katrin Streckfuss-Bömeke ${ }^{2}$; Gerd Hasenfuß ${ }^{2}$; Lars Maier'; Samuel Sossalla ${ }^{1}$

'Universitätsklinikum Regensburg, Innere Medizin II, Herzzentrum, Regensburg, Germany; ${ }^{2}$ Universitätsmedizin Göttingen, Klinik für Kardiologie und Pneumologie, Göttingen, Germany

Pharmacological approaches for treating atrial arrhythmias are limited. Recent genome wide association studies reported that the neuronal SCN10A Na-channel ( $\mathrm{NaV}_{1.8}$ ) might play a role in atrial electrophysiology. This study investigated the role of $\mathrm{NaV}_{1} .8$ in the human atria and its in-volvement in cellular electrophysiology and arrhythmogenesis.

Atrial myocardium from 34 patients with sinus rhythm was investigated. By using quantitative real-time PCR, we could confirm that NaV1.8 mRNA is expressed in human atrial myocardium ( $n=8$ patients) and found a $\sim 3.0$-fold higher mRNA expression compared to ventricular myocardium ( $n=10$ patients). Western Blot experiments confirmed the protein expression of $\mathrm{NaV}_{1} .8$ in the human atrium, which was less compared to the predominant heart isoform $\mathrm{NaV}_{1.5}\left(\mathrm{NaV}_{1.8} \mathrm{n}=14 \mathrm{pa}-\right.$ tients, NaV1.5: $\mathrm{n}=13$ ). Co-immunofluorescence staining revealed that $\mathrm{NaV} 1.8$ is expressed in atrial cardiomyocytes and localized in both t-tubules and intercalated discs. To evaluate the electrophysiological role of $\mathrm{NaV} 1.8$ we performed patch clamp experiments using isolated human atrial cardiomyocytes. Specific inhibition of $\mathrm{NaV}_{1.8}$ by the inhibitors A-803467 or PF-01247324 had no effects on action potential amplitude, resting membrane potential and upstroke velocity of atrial action potentials, while the action potential duration was slightly but not significant shortened ( $n=11-14$ cells from 5 patients each). However, we could demonstrate that $\mathrm{NaV}_{1.8}$ significantly contributes to the persistent "late" sodium current (INaL) and that inhibition of $\mathrm{NaV}_{1.8}$ by A-803467 ( $n=12$ cells/ 4 patients) or PF-01247324 ( $n=10 / 4)$ significantly reduces $\mathrm{INaL}$ compared to control $(n=15 / 6)$ in human atrial cardiomyocytes. Since INaL is linked to arrhythmia generation we studied the role of $\mathrm{NaV}_{1.8}$ in cellular arrhythmogenesis. Confocal line scans of human atrial cardiomyocytes loaded with the Ca indicator Fluo-4 showed that the proarrhythmic diastolic sarcoplasmic Ca leak could be significantly diminished by A-803467 $(n=73 / 9)$ or PF-01247324 $(n=88 / 11)$ compared to control $(n=84 / 13)$. Moreover, the incidence of major diastolic sarcoplasmic Ca release events as proarrhythmic triggers like Ca waves was significantly reduced by $\sim 68.8 \%$ after $\mathrm{NaV}_{1.8}$ inhibition (Control: $n=116 / 13, A-803467: n=90 / 9$, PF-01247324: $n=104 / 11)$. Accordingly, specific inhibition NaV1.8 with both novel agents ( $n=11 / 4$ each) potently reduced triggered activity (cellular afterdepolarizations and spontaneous action potentials) in patch clamp recordings of human atrial cardiomyocytes (Control: $n=14 / 4$ ).

This study demonstrates that $\mathrm{NaV}_{1} .8$ is expressed in the human atrium and contributes to INaL generation as well as cellular arrhythmogenesis. Importantly, selective inhibition of $\mathrm{NaV}_{1} .8$ with both novel inhibitors (PF-01247324 is orally bioavailable) reduces cellular arrhythmias. Thus, this study uncovers $\mathrm{NaV}_{1} .8$ as a new pharmacological approach for treating cellular arrhythmogenesis in the human atrium. 


\section{PS048}

proBNP als Marker zur Prognose von kardiovaskulären Erkrankungen bei nierentransplantierten Patienten

Elena Solovyeva'; Anja Scheuer ${ }^{2}$; Charlotte Zenner ${ }^{3}$; Insa Emrich; Johanna Hundsdorfer ${ }^{2}$; Lucie Bauer ${ }^{4}$; Danilo Fliser ${ }^{4}$; Michael Böhm ${ }^{5}$; Gunnar Heine ${ }^{6}$

${ }^{1}$ AGAPLESION Markus-Krankenhaus, Nephrologie, Frankfurt, Germany; ${ }^{2}$ Universitätsklinikum Homburg, Innere Medizin IV, Homburg, Germany; ${ }^{3}$ Agaplesion Markus Krankenhaus, Frankfurt, Innere Medizin II, Frankfurt, Germany; ${ }^{4}$ Universitätsklinikum des Saarlandes, Homburg, Innere Medizin IV, Homburg, Germany; ${ }^{5}$ Homburg, Innere Medizin III / Innere Medizin IV, Homburg, Germany; ${ }^{6}$ AGAPLESION Markus-Krankenhaus / Universitätsklinikum Homburg, Inneren Medizin II / Innere Medizin IV, Frankfurt, Germany ${ }^{\top}$ Universitätsklinikum Homburg, Innere Medizin III / Innere Medizin IV, Homburg, Germany

Hintergrund: Herz-Kreislauf-Erkrankungen sind die häufigste Todesursache bei Patienten nach Nierentransplantation: daher ist es von großer Bedeutung, bei diesen Patienten kardiovaskuläre Erkrankungen frühzeitig zu identifizieren. Unklar ist bisher, ob kardiale Biomarker wie proBNP bei nierentransplantierten Patienten - ähnlich wie in der nierengesunden Allgemeinbevölkerung - kardiovaskuläre Erkrankungen widerspiegeln oder aufgrund ihrer Akkumulation bei verminderter glomerulärer Filtrationsrate lediglich das Vorhandensein einer chronischen Nierenerkrankung reflektieren.

Wir wollen bei nierentransplantierten Patienten in verschiedenen Stadien der chronischen Nierenerkrankungen die Assoziation zwischen Plasma proBNP und echokardiographischen Parametern vor und nach Adjustierung für kardiovaskuläre Risikofaktoren, prävalente atherosklerotische kardiovaskuläre Erkrankungen (ASCVD) und glomeruläre Filtrationsrate untersuchen.

Methoden: In der HOMe ALONE Studie wurden 181 nierentransplantierte Patienten untersucht, bei denen bei Studieneinschluss proBNP gemessen wurde. Es wurden parallel konventionelle kardiovaskuläre Risikofaktoren (Alter, Geschlecht, arterieller Blutdruck, prävalenter Diabetes Mellitus, Plasma-Cholesterin, Nikotinkonsum), glomeruläre Filtrationsrate und prävalente ASCVD (definiert als koronare Herzerkrankung, zerebrovaskuläre Erkrankung und / oder peripheren arteriellen Verschlusskrankheit) erfasst und echokardiographisch Ejektionsfraktion (EF), linksventrikulärer Massenindex, linksatrialer Volumenindex und $\mathrm{E} / \mathrm{e}^{\prime}$ bestimmt.

Ergebnisse: Erwartungsgemäß stieg proBNP mit fortschreitenden Stadien der chronischen Nierenerkrankung an. So betrug das mediane proBNP bei CKD G 1 / 2205 pg/ml (Interquartilrange [IQR] 107; 518), bei CKD G 3a 275 pg/ml (IQR 125; 613), bei CKD G 3b 453 pg/ml (IQR 227; 1463) und bei CKD G 4 / 5902 pg/ml (IQR 346; 2132). In allen GFR-Stadien hatten Patienten mit vorbestehenden CVD zumindest tendenziell höheres proBNP im Vergleich zu Patienten ohne CVD ( $G_{1} / 2 p=0,03$; $\mathrm{G}$ за $\mathrm{p}=0,15 ; \mathrm{G}$ 3b $\mathrm{p}=0,09 ; \mathrm{G} 4 \mathrm{p}=0,05)$.

Bei univariater linearer Regressionsanalyse war proBNP nicht mit der Ejektionsfraktion assoziiert, was auf die geringe Anzahl von Patienten mit $\mathrm{EF}<60 \%$ zurückgeführt werden könnte. Hingegen zeigte sich in univariater Analyse eine Assoziation von proBNP mit den echokardiographisch bestimmten linksventrikulärem Massenindex, mit linksatrialem Volumenindex und mit E/e'. Diese Assoziationen blieben auch nach Adjustierung für die glomeruläre Filtrationsrate, für traditionelle kardiovaskuläre Risikofaktoren und für ASCVD signifikant.

Schlussfolgerungen: Unsere Daten bestätigen, dass proBNP bei nierentransplantierten Patienten zwar mit den Stadien der chronischen Nierenerkrankung G1-5 steigt, dennoch aber unabhängig mit echokardiographischen Schädigungsmarkern korreliert. Somit kann proB-
NP bei Patienten nach Nierentransplantation sinnvoll zur Diagnostik von kardiovaskulären Erkrankungen eingesetzt werden.

\section{PS049}

Wirkung von Spleen Tyrosin Kinase (Syk)-Inhibitoren auf synoviale Fibroblasten von Patienten mit rheumatoider Arthritis

Pierrette Lallah Missimana ${ }^{3}$; Magnus Diller'; Stefan Rehart ${ }^{2}$; Ulf Müller-Ladner'; Elena Neumann'

${ }^{1}$ Campus Kerckhoff, Justus-Liebig-Universität Gießen, Abteilung für Rheumatologie und Klinische Immunologie, Bad Nauheim, Germany; ${ }^{2}$ Agaplesion Markus Krankenhaus, Frankfurt, Abteilung für Unfallchirurgie, Frankfurt, Germany; ${ }^{3}$ Forschung Rheumatologie FGl, Bad Nauheim, Germany

Hintergrund: Die Spleen Tyrosin Kinase (Syk) ist eine intrazelluläre Proteinkinase, die in Immunzellen und nicht-häematopoetischen Zellen, wie z.B. Fibroblasten, in Signalübertragungsprozesse involviert ist. Verschiedene Syk-Inhibitoren werden daher aktuell in präklinischen und klinischen Studien, z.B. zur Behandlung von Neoplasien sowie von Autoimmunerkrankungen getestet. In präklinischen Studien zeigte der Syk-Inhibitor Fostamatinib eine ausgeprägte anti-inflammatorische Wirkung auf synoviale Fibroblasten (SF) von Patienten mit rheumatoider Arthritis (RA) [1]. Diese Zellen spielen in der Pathogenese der RA durch Vermittlung matrix-destruktiver Prozesse eine wichtige Rolle. Klinische Studien waren aufgrund der Nebenwirkungen und der damit limitierten Steigerung der Dosierung dagegen nur mäßig erfolgreich. Allerdings wurden inzwischen weitere Inhibitoren wie RO9021 oder TAK-659 entwickelt. Ob auch diese Inhibitoren eine entsprechend anti-inflammatorische Wirkung auf RASF vermitteln, wurde bisher nicht untersucht.

Ziele: Diese Studie untersuchte den Effekt von Syk-Inhibitoren auf die inflammatorische Antwort von RASF.

Methoden: Die RASF wurden aus RA-Synovialgewebe im Rahmen von Gelenkersatzoperationen isoliert. Die RASF wurden mit verschiedenen Konzentrationen von RO9021 und TAK-659 für $2 \mathrm{~h}$ vorbehandelt und für $17 \mathrm{~h}$ mit IL-1 $\beta$ oder für $24 \mathrm{~h}$ mit Onkostatin M (OSM) stimuliert. Anschließend wurden die Überstände abgenommen und die Konzentration von IL-6 mittels ELISA gemessen. Die Proliferation unter Syk-Inhibition wurde mittels eines BrdU-Assays bestimmt. Die mögliche Beeinflussung durch Apoptose-Induktion wurde anhand der Messung der Caspase 3/7 Aktivität untersucht.

Ergebnisse: Bei TAK-659 als auch bei RO9021 zeigte sich eine signifikante Reduktion der durch IL-1 $\beta$ induzierten Ausschüttung des pro-inflammatorischen Zytokins IL- 6 ab $10 \mu \mathrm{M}$. TAK- 659 wies bei $10 \mu \mathrm{M}$ mit $48 \%(p<0,01)$ eine stärkere Reduktion auf als RO9021 mit 26\% $(P<$ $0,05)$, jeweils verglichen mit IL-1 $\beta$ stimulierten RASF. Auch bei $15 \mu \mathrm{M}$ hemmte TAK-659 mit 69\% ( $p<0,001)$ stärker als RO9021 mit 35\% ( $p$ $<0,001$ ). Die durch OSM ausgelöste IL-6-Ausschüttung wurde durch TAK-659 ab $10 \mu \mathrm{M}$ signifikant gehemmt $(85 \%$ bei $10 \mu \mathrm{M}, \mathrm{p}<0,05)$.

Die Proliferation unstimulierter RASF wurde durch beide Syk-Inhibitoren ab $5 \mu \mathrm{M}$ gesenkt. Die maximalen Effekte konnten mit 78\% (p $<0,01)$ bei $15 \mu \mathrm{M}$ TAK-659 und mit $62 \%(\mathrm{p}<0,01)$ bei $15 \mu \mathrm{M}$ RO9021 beobachtet werden.

Diese Effekte wurden nicht durch Induktion von Apoptose vermittelt $(n=3)$.

Schussfolgerungen: Die Syk-Inhibitoren RO9021 und TAK-659 reduzieren die IL-1 $\beta$-induzierte Ausschüttung von IL- 6 bei RASF. Der Effekt scheint nicht nur auf den IL-1 $\beta$ Signalweg begrenzt zu sein, da auch die Aktivierung durch OSM und die zelluläre Proliferation gehemmt 
wird. Sollten entsprechende Konzentrationen in vivo erreicht werden können, bieten auch diese Syk-Inhibitoren die Möglichkeit einer Modulation des aggressiven Phänotyps von RASF.

[1] Cha et al., J Pharmacol Exp Ther. 2006

\section{PS050 \\ Wirkung von Visfatin auf das osteogene Potential mesenchymaler Stromazellen}

Dennis Küppers

Campus Kerckhoff, Justus-Liebig-Universität Giessen, Rheumatologie und klinische Immunologie, Bad Nauheim, Germany

Hintergrund: Chronische degenerative Erkrankungen des Knochens wie Osteoarthritis (Arthrose/OA) und Osteoporose resultieren für viele Patientinnen und Patienten in chronischen Schmerzen und körperlichen Einschränkungen. Mesenchymale Stromazellen (MSCs) spielen eine wichtige Rolle bei der Aufrechterhaltung und Regeneration gesunder Knochen und Gelenke. Adipokine wie Visfatin beeinflussen die Leistungsfähigkeit von MSCs und können so zur Entstehung von Osteoporose beitragen. Wir untersuchten in unserer Studie eine long non-coding RNA (IncRNA). LncRNAs sind lange Ribonucleotid-Moleküle, die nicht für Proteine kodieren, jedoch direkt mit DNA und RNA als auch mit Proteinen interagieren und so eine regulatorische Brücke schlagen können. Eine Beteiligung der IncRNA H19 wurde bereits bei verschiedenen Tumorerkrankungen und während der Embryonalentwicklung nachgewiesen. Im Rahmen der osteogenen Differenzierung von MSCs zeigte sich eine kontinuierliche Hochregulation von $\mathrm{H}_{191}$ wobei es über TGF $\beta 1$ und $\mathrm{Wnt} / \beta$-Catenin das osteogene Potential von MSCs verstärkte2,3.

Methoden: MSCs von Gesunden (hMSCs) und von OA-Patienten (phMSCs) wurden in einem Konditionierungsmedium mit/ohne Visfatin über 21 Tage zur osteogenen Differenzierung angeregt. Die Matrixmineralisierung (Tag 21) wurde mittels Alizarin Red Färbung nachgewiesen. An den Tagen 2,7 und 14 wurde die Expression von IncRNA H19 mittels Realtime PCR des Zelllysats und von IL-6 im Überstand mittels ELISA bestimmt.

Ergebnisse: In der unstimulierten Differenzierung war $\mathrm{H}_{19}$ über die Zeit kontinuierlich heraufreguliert. Zusätze von Leptin, Resistin oder TNFa veränderten diese Regulation nicht. Wurde jedoch Visfatin zugesetzt, zeigte sich eine bis zu 10-fache signifikant herabgesetzte H19-Expression (phMSC: Tag 7, p=0,03, Tag 14, $p=0,002$ ). MSCs, die mit Visfatin differenziert wurden, zeigten eine erhöhte Mineralisierung und höhere IL- 6 Level als unstimulierte Kontrollen. Die Zugabe von TNF steigerte die IL-6 Level, jedoch nicht die H19-Expression und Matrixmineralisierung.

Diskussion: IncRNA H19 wurde während der MSC-Differenzierung durch Visfatin signifikant herabreguliert. Visfatin könnte so regional in entzündetem Gewebe zu einem verringerten osteogenen Effekt der H19 und letztlich zur Entstehung degenerativer Erkrankungen führen. Da diese Wirkung nicht bei anderen Adipokinen (Leptin, Resistin) oder dem potenten Entzündungsmediator TNF囚 nachweisbar war, ist hier von einem Visfatin-spezifischen Effekt auszugehen.

[1] Wang, L. Differential expression of long noncoding ribonucleic acids during osteogenic differentiation of human bone marrow mesenchymal stem cells. Int. Orthop. 39, 1013-1019 (2015).

[2] Huang, Y. Long Noncoding RNA H19 Promotes Osteoblast Differentiation Via TGF-?1/Smad3/HDAC Signaling Pathway by Deriving miR675. Stem Cells 33, 3481-3492 (2015).
[3] Liang, W.-C. H19 activates Wnt signaling and promotes osteoblast differentiation by functioning as a competing endogenous RNA. Sci. Rep. 6, 20121 (2016).

\section{PS051 \\ Modulation des aggressiven Phänotyps von synovialen Fibroblasten bei rheumatoider Arthritis durch Peficitinib}

Magnus Diller'; Rebecca Hasseli'; Marie-Lisa Hülser'; Iris Aykara'; Stefan Rehart $^{2}$; Ulf Müller-Ladner'; Elena Neumann ${ }^{1}$

${ }^{1}$ Campus Kerckhoff, Justus-Liebig-Universität Gießen, Abteilung für Rheumatologie und Klinische Immunologie, Bad Nauheim, Germany; ${ }^{2}$ GGAPLESION Markus-Krankenhaus, Abteilung für Orthopädie und Unfallchirurgie, Frankfurt, Germany

Einleitung: Tofacitinib und Baricitinib waren die ersten Vertreter ihrer Substanzklasse, der Januskinase-Inhibitoren (JAKi), die zur Behandlung der rheumatoiden Arthritis (RA) zugelassen wurden. Peficitinib, ein panJAKi und Filgotinib, ein $\mathrm{JAK}_{1 / 2}$-Inhibitor durchliefen bereits erfolgreiche Phase-III-Studien. Es konnte gezeigt werden, dass mit diesen neueren JAKi höhere maximale Serumkonzentrationswerte erreicht werden können [1, 2]. Über die Wirkung dieser beiden JAKi auf synoviale Fibroblasten (SF), welche primär nicht durch JAK-abhängige Stimuli, wie IL-1 $\beta$, aktiviert werden, ist bisher wenig bekannt. SF spielen aber in der Pathogenese der RA eine entscheidende Rolle, da sie einen aggressiven matrix-destruktiven Phänotyp annehmen (RASF).

Ziel: Diese Studie untersuchte den Effekt von Peficitinib im Vergleich zu anderen JAKi auf RASF.

Methoden: Synovialgewebe wurde von RA-Patienten im Rahmen von Gelenkersatzoperationen gewonnen. Aus dem Gewebe isolierte RASF wurden mit unterschiedlichen Konzentrationen von Peficitinib und Filgotinib inkubiert und mit IL-1 $\beta$ (10 ng/ml) stimuliert. Nach $17 \mathrm{~h}$ wurden die Überstände abgenommen und die Konzentration von IL-6 und MMP-3 mittels ELISA gemessen. Die Proliferationsrate der RASF unter JAKi wurde mittels Nachweises des BrdU-Einbaus in die DNA bestimmt. Die Migration der RASF hin zu einem FCS-Gradienten sowie die Kurzzeit-Adhäsion auf Plastik unter Einfluss der Inhibitoren wurde ebenfalls gemessen. Die mögliche Zytotoxizität von Peficitinib wurde durch Messung der Zellmembranintegrität über die Zeit bis zu 48h gemessen.

Ergebnisse: Peficitinib senkte die Ausschüttung des pro-inflammatorischen Zytokins IL- 6 um $62 \%$ bei $5 \mu \mathrm{M}(\mathrm{p}<0.001, \mathrm{n}=7$ ) und um $24 \%$ bei $1 \mu \mathrm{M}$. Filgotinib führte im Vergleich dazu nur zu einem Rückgang um $30 \%$ bei $5 \mu \mathrm{M}(\mathrm{p}<0.05)$. Auch die Ausschüttung von MMP-3 wurde nicht durch Filgotinib aber durch Peficitinib gesenkt ( $5 \mu \mathrm{M}, 88 \%, \mathrm{p}<$ o.001). Peficitinib reduzierte außerdem die Proliferationsrate der IL-1 $\beta$ aktivierten RASF um $23 \%$ bei $1 \mu \mathrm{M}(\mathrm{p}<0.05)$ während Filgotinib keinen Einfluss auf die Proliferation hatte. 1 und $5 \mu \mathrm{M}$ Peficitinib führten zu einem Rückgang der Migration der RASF um 38\% ( $p<0,001)$ bzw. 92\% $(p<0,001, n=4)$. Für die beobachteten Effekte von Peficitinib war die Zytotoxizität des JAKi nicht verantwortlich, da auch nach $48 \mathrm{~h}$ keine Veränderung der Zellmembranintegrität bei Konzentrationen bis zu 5 $\mu \mathrm{M}$ beobachtet werden konnte.

Schlussfolgerung: Peficitinib moduliert den aggressiven Phänotyp von RASF auch im entzündlichen Milieu und könnte daher besonders bei Patienten mit schweren synovialen Proliferationen therapeutisch von Vorteil sein.

[1] Vanhoutte et al., Efficacy, Safety, Pharmacokinetics, and Pharmacodynamics of Filgotinib, a Selective JAK-1 Inhibitor, After Short-Term 
Treatment of Rheumatoid Arthritis: Results of Two Randomized Phase Ila Trials. Arthritis Rheumatol 2017;69:1949-1959.

[2] Cao et al., Pharmacokinetics, Pharmacodynamics, and Safety of ASPo15K (Peficitinib), a New Janus Kinase Inhibitor, in Healthy Subjects. Clin Pharmacol Drug Dev 2016;5:435-449.

\section{PS052 \\ Völlig unzureichende Umsetzung der Endokarditis- prophylaxe(EP) im ambulanten Bereich bei Hochrisikopatienten mit angeborenen(AHF) und erworbenen HKL-Erkrankungen im Kindes- und Erwachsenenalter}

Richard Eyermann ${ }^{1}, 2$

'Dr. Eyermann München, Kinder- und Jugendmedizin, Kinderkardiologie, Sportmedizin, München, Germany; ${ }^{2}$ Klinik Schönsicht Berchtesgaden, Rehabilitation für Kinder und Jugendliche, AHB, Kind-Mutter / Vater-Rehabilitation,München, Germany

IE auch in D häufige schwere Erkrankung, hohe Morbidität u. Mortalität. Bei AHF Inzidenz 150x häufiger.

Methoden: In eigenen D-weiten zertifizierten Tagesfortbildungen (CME11/LZÄK) über „Rationale Antibiotikatherapie u. - prophylaxe in der zahnärztlichen Klinik u. Praxis bei Eingriffen im ZMK-Bereich sowie benachbarter Organsysteme" Wissen bei Zahnärzten jeweils vor Ganztagsseminaren standardisiert mittels Fragebogen erhoben (Endocarditis-Prophylaxis-in Interventions in Oropharynx, (EPIO)-Studie). Weitere Studiendaten einbezogen.

Ergebnisse: 384 Zahnärzte geprüft, 240 m., 144 W., Alter 25-64 J., 23 Kliniker, 361 Praktiker: @ Wissen um EP bei dentalen Eingriffen nur $38,6 \%$, min.18,75,\% u. max. 82,82\%, @ Unwissen 61,4\%, Kliniker nicht besser als Niedergelassene. Kenntnisse v.a. in Indikationen zur EP, kaum Wissen in Praxisumsetzung bei Risikostratifizierung von Patienten, um leitliniengerechte Präparate $u$. Dosierungen bei Kindern aber auch Erwachsenen, bei Penizillinunverträglichkeit. Als Antibiotika vorrangig Clindamycin, falls Amoxicillin Dosen von 1ug-10g/die genannt. Meist Gleichsetzung von EP u. Therapie dentaler Infektionen: @2/3 aller Zahnärzte setzen EP fort über 3-5 Tage u. länger. Kaum Wissen bzw. Über-/Fehlversorgung bei fehlender EP-Notwendigkeit, wie v.a. bei Z. n. PCl, Z.n. ACB. @1/2 aller Zahnärzte erhebt Anamnese nur schriftlich.

Neue Studie von Piper/DHZ Bad Oeynhausen (ESC): Neuere Leitlinien führten nicht zu mehr EP-Maßnahmen: bei Zahnärzten insgesamt noch weniger EP auch bei Hochrisiko-Patienten.

Studie vom Nationalen Register AHF (2017) bei 1211 EMAH aus D u. UK: Weltweit erstmalig Kenntnisse über IE u. Notwendigkeit EP bei 343 EMAH mit hohem IE-Risiko, 868 mit niedrigem IE-Risiko erhoben, belegte signifikante klinisch relevante Wissenslücken bezüglich IE u. Antibiotikaprophylaxe bei EMAH-Patienten. Schlussfolgernd sollte eine Diskussion über IE u. AB-Prophylaxe mit jedem EMAH-Patienten während regelmäßiger klinischer Kontakte stattfinden, um diese Wissenslücke zu schließen.

\section{Konklusion:}

1. $>60 \%$ Unwissen in der eigenen EPIO-Studie belegt erheblichen Aufklärungsbedarf $u$. Notwendigkeit von Schulungen zur Akzep$\operatorname{tanz} u$. leitliniengerechten Durchführung der EP in dieser ärztlichen Fachgruppe.

2. Konsequente Mundhygiene, gute Zahnpflege u. regelmäßige zahnärztlichen Kontrollen.
3. Patienten mit AHF, EMAH und Eltern müssen stets auf ausgestellte, aktualisierte, vorgelegte $u$. durch Zahnärzte akzeptierte Herzpässe sowie Leitlinienumsetzung in praxi achten $\mathrm{u}$. bestehen.

4. Wichtige humanmedizinische Aufgabe ist Erinnerung an EP bei jeder ärztlichen Konsultation ((Kinder)-Kardiologe, Fach-/Hausarzt, etc.).

5. Stets evidenzbasierte Leitlinienaktualisierung der Endokarditis bzw. EP ist zwar wichtig, reicht aber allein nicht für die Umsetzung der EP in praxi bei weitem nicht aus.

6. Abklärung der Fieberursache bei Patienten mit AHF.

7. Bei manifesten bakteriellen Infektionen konsequente leitliniengerechte antibiotische Therapie.

\section{PS053}

Wirkung von Biologika auf die IL-17A- und TNF-induzierte Zytokinsekretion synovialer Fibroblasten von Patienten mit rheumatoider Arthritis oder Psoriasisarthritis

Klaus Frommer'; Stefan Rehart ${ }^{2}$; Ulf Müller-Ladner ${ }^{1}$; Elena Neumann ${ }^{1}$

'Justus-Liebig-Universität Gießen, Abt. für Rheumatologie und Klinische Immunologie, Campus Kerckhoff, Bad Nauheim, Germany; ${ }^{2}$ Agaplesion Markus-Krankenhaus, Klinik für Orthopädie und Unfallchirurgie, Frankfurt, Germany

Hintergrund und Zielsetzung: Rheumatoide Arthritis (RA) und Psoriasisarthritis (PsA) sind Autoimmunerkrankungen, die sowohl Gemeinsamkeiten als auch Unterschiede aufweisen. Das Anti-IL-17 Biologikum Secukinumab erwies sich beispielsweise als effektiver beim Einsatz in PsA-Patienten gegenüber dem bei RA-Patienten. Das Anti-TNF Biologikum Adalimumab hingegen ist für beide Erkrankungen gleichermaßen indiziert. Synoviale Fibroblasten (SF) sind zentrale Effektorzellen in der Pathophysiologie der RA und PsA. Daher untersuchten wir, ob sich die Wirkung der zwei Zytokine IL-17A und TNF- $\bigotimes$ als auch die Wirkung der zugehörigen Biologika zwischen RASF und PsASF unterscheidet und auf diese Weise zu dem Unterschied beiträgt, welcher beim therapeutischen Ansprechen beobachtet wird. Des Weiteren wurde die Wirkung des IL-17A Homologs IL-17F analysiert.

Methoden: SF wurden aus Synovium von Patienten mit PsA oder RA isoliert, welche sich einer Gelenkersatzoperation unterzogen. SF von RA- und PsA-Patienten wurden jeweils mit IL-17A, IL-17F und TNF-a allein oder in Kombination stimuliert. Eine Dosis-Wirkungskurvenanalyse für IL-17A wurde durchgeführt. Die Biologika Secukinumab und Adalimumab wurden eingesetzt, um die Effekte auf die SF zu blockieren. Als Maß für die proinflammatorische Reaktion wurde die Sekretion des Zytokins IL- 6 herangezogen und mittels L- 6 ELISA bestimmt.

Ergebnisse: Sowohl RASF als auch PsASF zeigten eine Reaktion auf IL-17A (IL-17A: 13,7-fach vs 6,9 fach; $n=3$ ), während IL-17F allein bei keinem der SF-Typen eine Induktion der IL 6 Sekretion bewirkte. Wurden IL-17A und IL-17F jedoch in Kombination mit TNF-a eingesetzt, so erhöhten beide Isoformen die IL- 6 Sekretion aufgrund eines starken synergistischen Effekts merklich. Erstaunlicherweise waren diese Wirkungen bei RASF deutlich stärker ausgeprägt als bei PsASF (IL-17A: 544-fach vs 127 -fach, IL-17F: 54 -fach vs 27 -fach; $n=3$ ). Jedoch zeigten Adalimumab und Secukinumab bei RASF und PSASF ähnliche Effektivität hinsichtlich der Auslöschung des synergistischen Effekts von IL-17A in Verbindung mit TNF-a.

Schlussfolgerungen: Der Zelltyp der SF scheint nicht zu den Unterschieden im therapeutischen Ansprechen auf das Anti-IL-17A Biologi- 
kum Secukinumab beizutragen, da die Reaktion auf IL-17A allein und IL-17A in Verbindung mit TNF-a bei PsASF nicht stärker ausfiel als bei RASF. Darüber hinaus wies Secukinumab bei beiden SF-Typen ähnliche Effektivität auf. Die Daten legen zudem nahe, dass in einem proinflammatorischen Milieu mit erhöhten TNF-Spiegeln sowohl IL-17A als auch IL-17F eine Rolle bei der SF-vermittelten Pathophysiologie der PsA spielen und daher das therapeutische Targeting von TNF bei beiden Erkrankungen effektiv ist.

Anerkennung: Gefördert durch einen unresticted educational grant der Fa. Celgene.

\section{PS054 \\ Kardiopulmonale Funktionsdiagnostik bei Kindern und Jugendlichen mit Typ-1-Diabetes - Besonderheiten der körperlichen Belastbarkeit und Sporttauglichkeit sowie der Blutglukosereaktion}

Richard Eyermann',

'Dr. Eyermann München, Kinder- und Jugendmedizin, Kinderkardiologie, Sportmedizin, München, Germany; ${ }^{2}$ Klinik Schönsicht Berchtesgaden, Rehabilitation für Kinder und Jugendliche, AHB, Kind-Mutter / Vater-Rehabilitation, München, Germany

Problemstellung: Muskelarbeit ist älteste Behandlungsmethode, zusammen mit Diät u. Insulinsubstitution (JOSLINsche Trias) tragende Therapiesäule, u. wird, ergänzt durch Schulung u. Stoffwechselselbstkontrolle, zu einem wesentlichen therapeutischen Prinzip zur Stabilisierung der diabetischen Stoffwechsellage. Körperliche Aktivität u. Sport sind bedeutsam in der Minderung des Krankheitsbeeinträchtigungsgefühls sowie in der Intervention atherogener Risikofaktoren.

Patientengut und Methodik: Fahrradergometrische Untersuchungen bei 109 Typ-1-Diabetikern im Alter von 9-18 Jahren im kompensierten Stoffwechselzustand. Evaluierung der körperlichen Leistungsfähigkeit PWC 170 u. des Stoffwechselverhaltens unter Ausdauerbelastung. Erarbeitung praxisrelevanter Schlussfolgerungen für die Therapieoptimierung.

Ergebnisse und Konklusion: Bei Fehlen diabetesbedingter Gefäß veränderungen, weisen nach eigenen Untersuchungen zur PWC170, juvenile Patienten die gleiche kardiopulmonale Leistungsfähigkeit auf, wie stoffwechselgesunde Kinder. Reduzierungen der PWC sind Folge mangelnder Muskelkonditionierung durch ungerechtfertigte Schonung in Schulsport $u$. außerschulischer Sportaktivität u. nicht bedingt durch die Stoffwechselerkrankung selbst.

Die Stoffwechselreaktion auf Muskelarbeit wird determiniert durch Diabetestyp (in der Regel Typ-1, zunehmend aber leider auch Typ-2), Ausgangsmetabolismus, Injektionsort u. Zeit der Insulinierung, Insulinart u. Insulinmenge, letzte Nahrungszufuhr u. die Art und Dauer der körperlichen Belastung.

Muskelarbeit übt einen "dualistischen Effekt" auf den diabetischen Stoffwechsel aus. Bei Stoffwechselkompensation induzieren nach eigenen Untersuchungen präventivmedizinisch angestrebte Ausdauerbelastungen generell $u$. different ausgeprägt einen Blutglukoseabfall, der vorrangig mit der Höhe der Ausgangsglykämie sowie der maximalen Insulinwirkung positiv korreliert.

Die Insulininjektion in die arbeitende Muskulatur führt zu einer rascheren $u$. anhaltenderen Resorption $u$. Verteilung.

Nahezu alle Sportarten können betrieben werden. Abzuraten ist von plötzlichen, nicht kalkulierbaren Belastungen mit Risiko (Klettern,
Tauchen, etc.). Belastungsinduzierten Hypoglykämien sollte durch Insulinreduktion bzw. Zusatz-KHE (-2) sowie der Wahl des Injektionsortes nach der bevorzugten Sportart vorgebeugt werden. Glukose ist stets für den Notfall bereitzuhalten. Auf Späthypoglykämien -auch noch nachts- muß geachtet werden. Teilfreistellungen sind bei diabetischen Spätkomplikationen gerechtfertigt. Unter Stoffwechseldekompensation (BG ca. $>300 \mathrm{mg} / \mathrm{dl}$ ) verstärkt Sport jedoch die Hyperglykämie und Ketose und ist daher kontraindiziert. Erst nach Rekompensation ist wieder Sport erlaubt!

\section{PS055 \\ Management of Heart Failure (HFrEF) Patients by Primary Care Physicians in Germany: Baseline Data of the AURORA Registry}

Uwe Zeymer'; Susanne Hoyer ${ }^{2}$; Stephan Hupfer ${ }^{2}$; Robert Groves ${ }^{2}$

'Klinikum Luwigshafen, Herzzentrum, Ludwigshafen, Germany; ${ }^{2}$ Novartis Pharma GmbH, CardioM Clin Res, Nürnberg, Germany

Background: Sacubitril/valsartan (S/V) was approved to treat systolic heart failure (HFrEF) in Europe and US in 2015. In Germany, most HFrEF patients are managed by Primary Care Physicians (PCP). The prospective AURORA registry was therefore set up to characterize this cohort of PCP-treated HFrEF patients and to capture baseline factors associated with subsequent changes in $\mathrm{HF}$ therapy in a real-world setting.

Methods: A total of 220 Primary Care Physicians throughout Germany enrolled 1260 qualifying adult HFrEF patients between March 2016 and May 2018 in AURORA. Consenting patients treated with conventional HF therapy or with S/V therapy were to be followed up over 12 months to capture if the HF therapy was changed in the 28 days before or after the qualifying index visit. If PCPs deemed that no change in $\mathrm{HF}$ therapy was required, only index visit data was to be captured. Baseline data are presented here according to the treatment groups "conventional" HF therapy (CT) or S/V therapy (S/V) as per index visit.

Results: Baseline data here is from 761 patients assigned S/V treatment and 499 patients assigned CT. Patient ages and \% females were similar between the 2 groups: 72.4 vs 73.3 years (median), $28.4 \%$ vs. $32.9 \%$ females (ns) for S/V and CT groups respectively. Patients assigned S/V therapy had a higher \% of NYHA class III or IV: 67.4 vs 47.8 , $p<0.001$ and a greater rate of use of beta-blockers ( $71.6 \%$ vs $60.1 \%$, $\mathrm{p}<0.001)$ and use of mineralocorticoid receptor antagonists $(33.0 \%$ vs $24.8 \%, p=0.002$ ) compared with those assigned conventional HF therapy. Medical history was generally similar between groups at baseline for hypertension $86.0 \%$ vs $88.0 \%$, coronary heart disease $63.6 \%$ vs $61.5 \%$, atrial fibrillation $45.3 \%$ vs $41.7 \%$, stroke $10.1 \%$ vs $13.4 \%$, diabetes $47.0 \%$ vs $42.7 \%$ and COPD: $21.1 \%$ vs $17.8 \%$ (all $p=n s$ ). Depression was less common $7.9 \%$ vs $12.0 \%, p<0.02$, whereas history of previous cardiac interventions was more frequent at baseline in the S/V group: defibrillator implant $23.1 \%$ vs $16.4 \%, p=0.004$, CABG bypass $15.8 \%$ vs $11.6 \%, p=0.04$, coronary angioplasty $43.5 \%$ vs $37.5 \%, p=0.04$ and prior myocardial infarction $31.7 \mathrm{vs} 26.5 \% \mathrm{p}=0.05$.

Values above are per group-mean $\%$ unless stated and p-values: Fischer's exact test; $n s: p>0.05$.

Conclusions: AURORA registry allows inferences on the standard of care therapy as provided to heart failure patients by family physicians in Germany. AURORA baseline data suggests that NYHA class is important in PCPs' treatment decision to switch certain patients to ARNI* therapy with S/V.

*Angiotensin Receptor Neprilysin Inhibitor

NYHA: New York Heart Association 


\section{PS056}

Gegenüberstellung der Low-Density-Lipoprotein-Abschätzung mittels Friedewaldformel vs. Martin/Hopkins Formel in einer Kohorte chronisch nierenkranker Patienten

Anja Leah Scheuer'; Insa E Emrich'; Kyrill S Rogacev²; Elena Solovyeva; Hubert Scharnagl ${ }^{4}$; Seth S Martin ${ }^{5}$; Danilo Fliser'; Michael Böhm ${ }^{6}$; Gunther Marsche $^{7}$; Jürgen Geisel ${ }^{8}$; Gunnar $\mathrm{H} \mathrm{Heine}^{3}$

${ }^{1}$ Universitätsklinikum Homburg, Innere Medizin IV - Nephrologie, Homburg, Germany; ${ }^{2}$ Sana HANSE-Klinikum, Innere Medizin II - Kardiologie, Wismar, Germany; ${ }^{3}$ Agaplesion Markus Krankenhaus, Medizinischen Klinik II - Nephrologie, Hochdruck und Gefäßkrankheiten, Frankfurt (Main), Germany; ${ }^{4}$ Medizinische Universität Graz, Klinisches Institut für Medizinische und Chemische Labordiagnostik, Graz, Austria; ${ }^{5}$ Johns Hopkins University School of Medicine, Ciccarone Center for the Prevention of Cardiovascular Disease, Division of Cardiology, Baltimore, Maryland, United States; ${ }^{6}$ Universitätsklinikum Homburg, Innere Medizin III - Kardiologie, Homburg, Germany; ${ }^{7}$ Medizinische Universität Graz, Otto Loewi Forschungszentrum (für Gefäßbiologie, Immunologie und Entzündung), Graz, Austria; ${ }^{8}$ Universitätsklinikum Homburg, Klinische Chemie und Laboratoriumsmedizin, Homburg, Germany

Eine exakte Bestimmung des Plasma Low-Density-Lipoprotein (LDL)-Cholesterins ist für die leitliniengetreue Therapie kardiovaskulärer Risikopatienten von großer Bedeutung. Die bisherige annäherungsweise Errechnung mithilfe der Friedewaldformel ist bekanntermaßen insbesondere bei hohen Triglyceriden und niedrigem LDL-Cholesterin ungenau und wird mancherorten durch photometrische Messungen ersetzt („LDL-C M“). Neue Formeln bieten kostengünstige Alternativen, um LDL-Cholesterin genauer abzuschätzen. Da Menschen mit einer chronischen Nierenerkrankung (CKD) häufig Hypertriglyceridämien und Hypo-LDL-Cholesterinämien aufweisen, stellt sich in diesem Patientenkollektiv in besonderem Ausmaß die Frage, inwiefern die durch verschiedene Formeln bestimmten und direkt gemessenen Werte differieren.

In einer Kohorte von 598 chronisch nierenkranken Patienten (CKD G2 - G4 nach KDIGO) berechneten wir LDL-Cholesterin sowohl mithilfe Friedewaldformel („LDL-C F“; LDL-Cholesterin = Gesamtcholesterin - HDL-Cholesterin - Triglyceride/5) als auch unter Verwendung einer Abschätzung nach Martin/Hopkins (,LDL-C N"). Letztere lehnt sich an die Friedewaldformel an, teilt aber Triglyceride nicht konstant durch fünf, sondern patientenindividuell durch einen von 180 variablen Faktoren. Bei 55 Probanden wurden die Plasmaspiegel außerdem mittels eines enzymatischen Farbtests (UV-/VIS-Photometrie, COBAS, Roche) direkt bestimmt.

Bezüglich beider Methoden zeigte sich ein systematischer Messfehler mit der Tendenz zu falsch niedrigen Werten, welcher unter Benutzung der herkömmlichen Friedewaldformel deutlich größer ausgeprägt ist (mittleres $\Delta$ LDL-C F - LDL-C M: -17,4 mg/dl; [95 \% Kl: $(-31,6)-$

$(-2,4)]$, mittleres $\Delta$ LDL-C N - LDL-C M: -9,0 mg/dl [KI: $(-28,2)-10,1])$. Im Mittel betrug die absolute Differenz zwischen den beiden Formeln 6,2 $\pm 8,6 \mathrm{mg} / \mathrm{dl}$. Die Differenz der Cholesterinschätzung korreliert unerwarteterweise nicht mit der glomerulären Filtrationsrate $(r=-0,078, p$ $=0,056)$, aber erwartungsgemäß mit den Plasma-Triglyceriden

$(r=-0,993, p<0,001)$.

Teilt man Plasma LDL-Cholesterin in Anlehnung an die Leitlinien in Kategorien ein ( $<70 \mathrm{mg} / \mathrm{dl}, 70-99 \mathrm{mg} / \mathrm{dl}, 100-129 \mathrm{mg} / \mathrm{dl}$ und $\geq 130$ $\mathrm{mg} / \mathrm{dl}$ ), so werden überraschend viele Patienten $(38,2 \%)$ gegenüber der direkten Messung in falsche Kategorien klassifiziert. Bei Berechnung mittels der neuen Formel kann diese Zahl signifikant reduziert werden
$(34,5 \%)$.

Sollten unsere Ergebnisse in größeren Kollektiven bestätigt werden, so sollte auch bei CKD Patienten zukünftig auf die Friedewaldformel verzichtet werden und LDL-Cholesterin entweder direkt gemessen oder mittels der Martin/Hopkins-Formel geschätzt werden.

\section{PS057 \\ Vorhersagekraft der Cholesterin Efflux Kapazität für das Voranschreiten einer chronischer Nierenerkrankungen}

Anja Leah Scheuer ${ }^{1}$; Insa E Emrich ${ }^{2}$; Adam M Zawada ${ }^{2}$; Sabine Kern ${ }^{3}$; Akos Heinemann ${ }^{3}$; Kyrill S Rogacev ${ }^{4}$; Danilo Fliser ${ }^{2}$; Gunnar H Heine ${ }^{5}$; Gunther Marsche ${ }^{3}$

'Universitätsklinikum Homburg, Innere Medizin IV - Nephrologie, Homburg, Germany; ${ }^{2}$ Universitätsklinikum des Saarlandes, Innere Medizin IV - Nephrologie, Homburg, Germany; ${ }^{3}$ Medizinische Universität Graz, Otto Loewi Forschungszentrum (für Gefäßbiologie, Immunologie und Entzündung), Graz, Austria; ${ }^{4}$ Sana HANSE-Klinikum, Innere Medizin II - Kardiologie, Wismar, Germany; ${ }^{5}$ Agaplesion Markus Krankenhaus, Medizinischen Klinik II - Nephrologie, Hochdruck und Gefäßkrankheiten, Frankfurt (Main), Germany

Jüngste Studien in der Allgemeinbevölkerung zeigen, dass die Funktionalität des High-Density-Lipoproteins (HDL) - etwa in Form der Cholesterin Efflux Kapazität (CEC) - eine weitaus größere prognostische Rolle in Bezug auf die Vorhersage kardiovaskulärer Ereignisse spielt als dessen bloße Quantität. Patienten mit chronischer Nierenerkrankung haben ein erhöhtes Risiko für das Auftreten kardiovaskulärer und renaler Ereignisse. In einer prospektiven Kohortenstudie erkannten wir kürzlich, dass die CEC bei chronisch nierenkranken Patienten - anders als in der Allgemeinbevölkerung - kein unabhängiger Prädiktor für kardiovaskuläre Ereignisse ist. Ergebnisse der $4 \mathrm{D}$ Studie bestätigten diese Beobachtung bei Hämodialysepatienten. Unklar blieb, welche Bedeutung die CEC als Prädiktor der Progredienz der chronischen Nierenerkrankung hat, nachdem eine einzelne Kohortenstudie bei nierentransplantierten Patienten renoprotektive Effekte einer hohen CEC postulierte.

In unserer Kohortenstudie wurden 526 chronisch nierenkranken Patienten mit CKD G2 bis G4 zum Einschlusszeitpunkt untersucht (geschätzte glomeruläre Filtrationsrate [eGFR] 15-89 ml/min/1,73 $\mathrm{m}^{2}$ ) und die CEC als Cholesterin-Aufnahmekapazität von Apolipoprotein Bdepletiertem Serum aus J 774 Makrophagen bestimmt. Als kombinierter primärer renaler Endpunkt war definiert: eine Halbierung der GFR, die Initiierung einer Nierenersatztherapie und Tod. Die statistische Auswertung erfolgte mittels Kaplan-Meier Analysen (nach Stratifizierung der Patienten in Tertile) und mittels Cox-Regressionsanalyse (mit Korrektur für klassische kardiovaskuläre und renale Risikofaktoren).

Bei Studieneinschluss korrelierte die CEC moderat mit HDL-Cholesterin $(r=0,350 ; p<0,001)$ und minimal mit der eGFR $(r=0,065 ; p=$ 0,028). Überraschenderweise wiesen Patienten mit der höchsten CEC eine raschere Progredienz der chronischen Nierenerkrankung auf (Kaplan-Meier Analyse mit log-rank Test: $p=0,002$ ). Diese Assoziationen blieben nach Korrekturen für renale und kardiovaskuläre Risikofaktoren signifikant (Cox-Regressionsanalyse: Hazard Ratio per \%: 1,123 [Cl $1,035-1,219])$.

Während eine hohe CEC somit für nierengesunde Patienten prognostisch günstig bezüglich kardiovaskulärer Ereignisse sind, ist dieser Zusammenhang bei CKD-Patienten nicht gegeben. Paradoxerweise ist eine erhöhte CEC darüber hinaus mit einer größeren Wahrscheinlichkeit, einen renalen Endpunkt zu erreichen, vergesellschaftet. 


\section{PS058}

\section{Starvation Based Differential Virotherapy}

\section{Gabriel Scheubeck'; Susanne Berchtold ${ }^{2}$; Ulrich Lauer ${ }^{2}$}

${ }^{1}$ Klinikum der Universität München - Großhadern, Innere Medizin, Hämatologie und Onkologie, München, Germany; ${ }^{2}$ Medizinische Universitätskliniken Tübingen, Innere Medizin VIII Klinische Tumorbiologie, Tübingen, Germany

a) Starvation has been shown to sensitize tumor cells to chemotherapy whilst protecting normal cells at the same time [Differential Stress Resistance]. The ability of oncolytic virotherapeutics (OVs) to selectively infect, replicate in and lyse cancer cells outlines a promising approach in cancer therapy. We hypothesized that starvation would increase the oncolytic potential of OVs in colorectal carcinoma (CRC) cells and protect normal colon cells against virus-mediated cell lysis.

b) Three different human CRC cell lines (HT-29, HCT-15 and HCT-116) as well as two human normal colon cell lines (CCD-18 Co and CCD$841 \mathrm{CoN}$ ) were subjected to various starvation regimes in glucose and/or serum restricted cell culture medium and infected with two state-of-the-art OVs [i.e., measles vaccine virus (MeV) and vaccinia virus (GLV-1h68)]. Fasting regimes applied were either short-term starvation ( $24 \mathrm{~h}$ pre-infection) or long-term starvation ( $24 \mathrm{~h}$ preand $96 \mathrm{~h}$ post-infection). We used cell viability assays to determine the cell killing capabilities of i) viro-therapy, ii) starvation, and iii) the combination of these two. Virus growth curves were generated to assess the replication of MeV in starved and non-starved HT-29 cells.

c) Infection of starved cancer cells exhibited additional oncolytic potential of virotherapy plus starvation for most combinations. Remarkably, long-term low serum/ standard glucose starvation potentiated the efficacy of MeV-mediated cell killing in HT-29 CRC cells, whereas it was decreased in normal colon cells CCD-18 Co and CCD-841 CoN. Interestingly, viral replication of MeV in HT-29 was decreased in long-term starved cells, but was increased after shortterm low glucose/ low serum starvation.

d) In conclusion, starvation based virotherapy could enhance the oncolytic effect on CRC in future anti-cancer therapy while protecting normal tissues from side effects.

\section{PS059 \\ Direkte Interaktion von E. coli zum Darmepithel mit destabilisierten Zell-Zell-Kontakten begünstigt die Entstehung einer SBP}

Marika Haderer; Heidi Gschwendtner; Elisabeth Aschenbrenner; Kristin Pollinger; Sophie Schlosser; Claudia Kunst; Karsten Gülow; Martina Müller-Schilling

Universitätsklinikum Regensburg, Klinik und Poliklinik für Innere Medizin I, Gastroenterologie, Endoskopie Rheumatologie und Infektiologie, Regensburg, Germany

Einleitung: Die Leberzirrhose wird als sogenannte end-stage Lebererkrankung angesehen. In diesem Zustand ist die Leber durch einen Umbau des Bindegewebes mit knotiger Struktur gekennzeichnet und die Hepatozyten sind in ihrer Funktion deutlich eingeschränkt. Die Leberzirrhose ist mit einem Verlust der Diversität der Darmflora vergesellschaftet. Daneben verändern sich die intestinale Barriere und die Immunantwort im Sinne einer systemisch multifaktoriellen Immunsuppression. Aszites und spontan bakterielle Peritonitis (SBP) sind Folgen einer Leberzirrhose, welche mit zunehmender Schwere der Erkrankung vermehrt auftreten. Maßgebend für die Entstehung einer SBP ist die bakterielle Translokation von darmständigen Bakterien in das splanchnische Abstromgebiet. In diesem Zusammenhang werden die Rollen des Mikrobioms, der Darmbarriere und der intestinalen Immunantwort diskutiert. Die molekularen Mechanismen dieses Prozesses sind bisher nicht geklärt. Klinisch ist das Erscheinungsbild der SBP mit einer 1-Jahres Mortalität von $70 \%$ jedoch höchst relevant.

Ziel: Vergleichend zu Darmbiopsien von Leberzirrhosepatienten wurden im Caco-2 in-vitro Modell die Regulationen von Zell-Zell-Kontakten nach der Stimulation mittels Bakterien untersucht und trans/ intrazelluläre Translokationswege evaluiert.

Methodik: Untersucht wurden Darmbiopsien von 20 Kontroll- und 8 Leberzirrhosepatienten (4x Child A, 4x Child C). Die Stärke der Mukusschicht wurde in Kryoschnitten mittels Alcian Blau Färbung ermittelt. Die Mengen an Zellkontaktkomponenten (Occludin und E-cadherin) wurde auf Proteinebene via Immunhistochemie bestimmt. Vergleichend wurde die humane Darmepithelzelllinie Caco-2 nach 6 Tagen Zellwachstum mit aus Patienten isolierten SBP Keimen (E. coli) in verschiedenen $\mathrm{MOI}$ für $4 \mathrm{~h}$ koinkubiert. Die Regulation von E-cadherin und Occludin wurde im (in)direkten Kontakt via Transwell im Westernblot analysiert.

Ergebnis: Leberzirrhosepatienten wiesen im Vergleich zu den Kontrollen $(16,4 \mu \mathrm{m})$ mit einer durchschnittlichen Stärke von $12,8 \mu \mathrm{m}$ eine reduzierte Mukusschicht auf. Ebenso zeigten sich vor allem bei Patienten mit SBP im Verlauf deutliche Veränderungen im Occludin- und E-cadherin-Gerüst. Die Koinkubation von Caco-2 Zellen mit E. coli SBP Keimen führte zu unterschiedlich stark ausgeprägten Reduktionen von Occludin und E-cadherin. Ein direkter Kontakt von Bakterien und Epithelzellen begünstigte die Destabilisierung des Darmepithels.

Schlussfolgerung: Im Rahmen einer Leberzirrhose kommt es mukosal sowohl zu einer reduzierten Mukusschicht als auch zu destabilisierten Zell-Zell-Kontakten. Diese massiven epithelialen Defekte können auch durch das intestinale Immunsystem nicht kompensiert werden. Die Kombination der mukosalen Defekte erleichtert somit die bakterielle Translokation und fördert die Entstehung einer SBP.

PS060

Sham-controlled trials for invasive interventions in cardiovascular medicine - A systematic review and meta-analysis

Lucas Lauder'; Sean Scholz ${ }^{2}$; Sebastian Ewen ${ }^{2}$; Christian Ukena²; Michael Böhm²; Felix Mahfoud ${ }^{2}$

'Universitätsklinikum des Saarlandes, Klinik für Innere Medizin III Kardiologie, Angiologie und Internistische Intensivmedizin, Homburg, Germany; ${ }^{2}$ Universitätsklinikum des Saarlandes und Medizinische Fakultät der Universität des Saarlandes, Klinik für Innere Medizin III - Kardiologie, Angiologie und Internistische Intensivmedizin, Homburg, Germany

Background: In the light of technical progress achieved in cardiovascular medicine, invasive interventions have gained importance in everyday practice. Although randomized placebo-controlled trials are considered the gold standard for discriminating between true treatment and placebo effects in the development of new drugs, randomized sham-controlled trials of invasive interventions are still rare in cardiovascular medicine. 
Methods: We searched MEDLINE and Web of Science for double-blinded, randomized, sham-controlled trials of invasive interventions in cardiology and cardiovascular surgery. All records from their inception to October 2018 were considered. Owing to the heterogeneity of the studies, effect size (ES) was calculated to assess the biological efficacy of the active and sham treatment on continuous efficacy endpoints. ES were graduated according to Cohen's rule of thumb (ES < 0.5 was considered small, ES 0.5-0.79 moderate, ES > 0.8 large). A meta-analysis was performed to assess the blood-pressure-lowering efficacy of catheter-based renal denervation in sham-controlled trials (RDN).

Results: A total of 27 trials that enrolled 3,179 patients were included. The majority of studies (56\%) were published during the last four years. Eight trials (28\%) met all predefined primary efficacy endpoints. Invasive interventions achieved a large and moderate ES in $30 \%(3 / 10)$ each of the continuous endpoints, whereas the ES of a sham procedure was large in $10 \%(1 / 10)$ of the continuous endpoints. None of the sham procedures had a moderate ES. A deterioration of the continuous primary outcome was not documented following active treatment but one (10\%) sham procedure.

A meta-analysis of sham-controlled trials investigating RDN in hypertension showed a more pronounced reduction in office (mean difference (MD) $-5.72 \mathrm{mmHg}$, 95\% confidence interval (Cl) -8.29 to -3.15 $\mathrm{mmHg}$ ), daytime (MD $-4.06 \mathrm{mmHg}, 95 \% \mathrm{Cl}-6.23$ to $-1.9 \mathrm{mmHg}$ ) and 24-hour ambulatory ( $\mathrm{MD}-3.57 \mathrm{mmHg}, 95 \% \mathrm{Cl}-5.23$ to $-1.91 \mathrm{mmHg}$ ) systolic blood pressure following RDN ( $n=582$ ) when compared with sham $(n=395)$.

Conclusion: ES of sham procedures were remarkable and underline the influence of non-specific mechanisms such as placebo on treatment effects. This meta-analysis adds to the growing body of evidence indicating the blood pressure-lowering efficacy of RDN.

\section{PS061}

Endoskopische Submukosa-Dissektion im distalen Anorektum: Effektivität, Sicherheit und unerwartete Nebenwirkungen sowie Notwendigkeit einer umfassenden Patientenaufklärung

Martin Raithel ${ }^{1}$; Alexander Hagel2 ${ }^{2}$; Andrea Anneken ${ }^{3}$; Heinz Albrechtt ${ }^{4}$; Francesco Vitali ${ }^{5}$; Alfred Brütting ${ }^{6}$

${ }^{1}$ Waldkrankenhaus St. Marien Erlangen, Medizinische Klinik II, Erlangen, Germany; ${ }^{2}$ Praxisklinik Schwabach, Gastroenterologie, Schwabach, Germany; ${ }^{3}$ Malteser Waldkrankenhaus St. Marien Erlangen, Medizinische Klinik II, Erlangen, Germany; ${ }^{4}$ Gemeinschaftspraxis Internisten am Ring Nürnberg, Gastroenterologie, Nürnberg, Germany; ${ }^{5}$ Uniklinikum Erlangen, Medizinische Klinik ${ }^{1}$, Erlangen, Germany; ${ }^{6}$ Malteser Waldkrankenhaus St. Marien Erlangen, Chirurgie, Erlangen, Germany

Hintergrund: Endoskopische Resektionen von Rektumneoplasien können durch EMR, ESD, eFTRD, transanale endoskopische Resektion (TEM) oder ein offenes chirurgisches Verfahren, das eine Stomakonstruktion erfordert, erreicht werden.

Diese Studie analysierte die Ergebnisse der ESD bei Rektumtumoren die in der Hämorrhoidalzone oder innerhalb einer Entfernung von $3 \mathrm{~cm}$ von der L. dentata lokalisiert waren, in Bezug auf die Resektabilität, Histologie, Komplikationen, Hämorrhoidalblutungen sowie andere seltene Ereignisse.

Materialien und Methoden: 46 Pat. mit rektalen Neoplasien und Hämorrhoiden Grad I-III wurden nach interdisziplinärem Konsens mit ESD behandelt. Die Tumoren befanden sich entweder im distalen Rectum (innerhalb von $3 \mathrm{~cm}$ von L. dentata, $\mathrm{n}=22$ ) oder in dem Anal- kanal $(n=24)$. Die ESD wurde mit oder ohne Hyaluronsäure durchgeführt, wobei dual knife, IT- oder Hakenmesser, Coaggrasper und der BOWA-Hochfrequenzgenerator (Arc 400, GastroCut-Modi) sowie TTSoder OTS-Clips bei Bedarf verwendet wurden.

Ergebnisse: 51 ESDs wurden bei 46 Pat. durchgeführt. Die rektalen Neoplasien enthielten typische Läsionen ( $n=37$ Pat., Adenome, Adenokarzinome), aber bei 8 Pat. (17.4\%) auch unerwartete Befunde mit kloakogenen Polypen, Kondylomen und fibroepithelialen Polypen mit sessilen serratierten Adenomen. Insgesamt konnte eine Ro-Resektion bei 36/46 Pat. (78.2\%; Größe 13-174 mm) erreicht werden. Die HF-Einstellungen waren GastroCUT (Effekt 3 + 3, Schnittgeschwindigkeit mittel oder schnell), wobei jeder Eingriff während der ESD individuell an Blutungsschwere, Schnittort (Sphinkterkontraktion, Hämorrhoide) oder Notwendigkeit einer Antikoagulation angepasst wurde.

Die Majorkomplikationen (1 Perforation, 4 Blutungen aus Rektumulkus) waren selten, aber es traten auch unerwartete Nebenwirkungen auf (Blasenentleerungsstörung, Hämaturie, femorales Hautemphysem). Überraschend war, dass keine sofortige oder verzögerte Hämorrhoidalblutung nach ESD auftrat, was möglicherweise auf den höheren verwendeten Koagulationsanteil beim Schneidestrom zurückzuführen ist.

Schlussfolgerung: ESD mit High-Volume-Injektion, Hyaluronsäure und erhöhten Koagulationsstromeinstellungen in der Hämorrhoidalzone vermeidet Blutungen nach ESD im distalen Rectum. Die ESD in diesem besonderen Bereich zeigt jedoch einige unerwartete Befunde wie Blasenentleerungsstörung, sexuell übertragbare Krankheit und kloakogene Polypen mit bösartigem Potential.

\section{PS062}

Detektion von lokalen, intestinal gebildeten IgE-Antikörpern gegen Lebensmittel bei Reizdarm und Effekt einer Antigenspezifischen Diät auf den Reizdarm (IBS)-Typ, Schweregrad und Symptome

Martin Raithel'; Eugenia Krischke²; Jürgen Kressel ${ }^{2}$; Frederik Krischke ${ }^{3}$; Heinz Albrecht ${ }^{4}$; Katharina Hotfiel ${ }^{2}$; Wolfgang Schultis ${ }^{5}$; Alexander Hagel ${ }^{6}$

${ }^{1}$ Waldkrankenhaus St. Marien Erlangen, Medizinische Klinik II, Erlangen, Germany; ${ }^{2}$ Malteser Waldkrankenhaus St. Marien Erlangen, Medizinische Klinik II, Erlangen, Germany; ${ }^{3}$ Uniklinikum Erlangen, Anästhesie, Erlangen, Germany; ${ }^{4}$ Gemeinschaftspraxis Internisten am Ring Nürnberg, Gastroenterologie, Nürnberg, Germany; ${ }^{5}$ Synlab Medizinisches Versorgungszentrum, Weiden/Opf., Weiden/Opf., Germany; ${ }^{6}$ Praxisklinik Schwabach, Innere Medizin, Schwaba, Germany

Hintergrund und Ziele: Die Diagnose des Reizdarms (IBS) basiert auf dem Vorliegen charakteristischer darmbezogener Symptome ohne pathologische Anomalien und erfordert den Ausschluss von Differentialdiagnosen. In dieser Studie wurde nach dem Vorliegen einer Gl-Allergie gesucht, indem das intestinale IgE in der endoskopisch gesteuerten Darmlavage gemessen und durch Vermeidung der verursachenden Nahrungsmittelallergene behandelt wurde.

Methoden: Von 60 konsekutiven Patienten erfüllten 36 die Rom III-Kriterien. Alle Patienten hatten eine Koloskopie mit segmentaler Lavage, um IgE-Antikörper gegen häufige Nahrungsmittelallergene nachzuweisen. IgE-positive Teilnehmer entfernten die jeweiligen Nahrungsmittel aus ihrer Ernährung. Anschließend wurden IBS-Symptome, Schweregrad und Rom III vor und nach Antigenvermeidung untersucht.

Ergebnisse: $30 / 36$ Patienten (83\%) wurden IgE-positiv (U/mg Protein) in der segmentalen Lavage $(>0,15)$ getestet, $47 \%$ von ihnen zeigten 
signifikant erhöhte $\lg E>0,35$, vs $25 \%$ bei Kontrollen. Intestinales $\lg E$ (Median) war signifikant höher bei IBS $(0,08 \mathrm{U} / \mathrm{mg}, 0,01-0,45)$ im Vergleich zu Kontrollen o,02U/mg; o,00-0,11, $\mathrm{p}<0,03)$.

Erhöhtes IgE wurde gegen Weizenmehl (33\%), Roggenmehl (11,1\%), Rindfleisch, Nüsse und andere Allergene gefunden. Der IBS-SSS korrelierte nicht mit der gemessenen IgE-Menge.

Nach der Diät zeigten 23\% eine fast vollständige Remission der Symptome, und 50\% zeigten eine deutliche Linderung mit einer $46 \%$-Reduktion der IBS-Symptome. Nicht-Atopiker reagierten am besten auf die Eliminationsdiät. In allen IBS-SSS-Kategorien konnte eine signifikante Verbesserung ( $p<0,0001)$ erreicht werden. Während die IBSD-Gruppe zuvor die dominierende Gruppe war, machte sie nach der Diät nur noch $27 \%$ aus.

Diskussion: Die endoskopisch gesteuerte segmentale Lavage ermöglicht den Nachweis von lokalem intestinalem IgE, welches eine effektive Erweiterung der gängigen Allergiediagnostik-Tools darstellt. Lokal produziertes lgE erwies sich bei $73 \%$ der IBS-Patienten als klinisch nachweisbar, bestätigte die Nahrungsmittelsensitivität in dieser Population und zeigt den hohen Bedarf an individuell angepassten Diäten.

\section{PS063 \\ Therapieintensivierung nach initialer Metforminbehandlung bei erwachsenen Patienten mit Typ-2-Diabetes - Ergebnisse aus den Registern DPV und DIVE}

Stefanie Lanzinger'; Bettina Hartmann ${ }^{2}$; Peter Bramlage ${ }^{3}$; Frank Jürgen Wosch ${ }^{4}$; Messut Durmaz ${ }^{5}$; Jochen Seufert ${ }^{6}$; Reinhard W. Holl'

'Universität Ulm, Institut für Epidemiologie und medizinische Biometrie, ZIBMT, Ulm, Germany; ${ }^{2}$ Klinikum Ludwigshafen, Medizinische Klinik C, Ludwigshafen, Germany; ${ }^{3}$ Institut für Pharmakologie und Präventive Medizin, Institut für Pharmakologie und Präventive Medizin, Cloppenburg, Germany; ${ }^{4}$ Diabetespraxis Wosch, Allgemein - und innere Medizin, Diabetologie, Ernährungsmedizin und Akupunktur, Hanau, Germany; ${ }^{5}$ Schwerpunktpraxis für Diabetes, Hormone und Stoffwechsel, Facharztpraxis für Innere Medizin, Endokrinologie \& Diabetologie, Hof, Germany; ${ }^{6}$ Universitätsklinikum Freiburg, Abteilung Endokrinologie und Diabetologie, Klinik für Innere Medizin II, Freiburg, Germany

Fragestellung: Welche Therapiestrategien folgen nach initialer Metformin-Monotherapie bei Erwachsenen mit Typ-2-Diabetes? Wie hoch ist der Anteil des Wechsels zu einer Insulintherapie?

Methoden: In den Diabetesregistern DPV und DIVE sind aktuell 605.772 (09/2018) Patienten dokumentiert. Davon wurden 69.185 Patienten $\geq 18$ Jahre mit Typ-2-Diabetes und initialer Metformin-Monotherapie untersucht, die seit Januar 2000 in DPV oder DIVE registriert sind. In Abhängigkeit vom weiteren Therapieverlauf wurden die Patienten in folgende Untergruppen eingeteilt: 1) fortlaufende Metformin-Monotherapie, Therapieintensivierung mit 2) oralen Antidiabetika (OAD) oder GLP-1 Analoga (ohne Insulintherapie), 3) einer Basalinsulin unterstützten oralen Therapie (BOT oder BOT plus $1 x$ täglich kurzwirksames Insulin), 4) sonstiger Insulintherapie (supplementäre Insulintherapie (SIT), Basal-Bolus-Therapie, Mischinsulintherapie).

Ergebnisse: Von den 69.185 untersuchten Typ-2-Diabetes Patienten blieben $51.177(74,0 \%)$ über einen durchschnittlichen Zeitraum von 0,9 Jahren bei einer Metformin-Monotherapie. Bei 7.095 (10,3\%) wurde die Therapie innerhalb von 3,6 Jahren (Zeitraum erster Termin mit Metformin bis zum aktuellsten Termin mit Intensivierung) mit einem zusätzlichen OAD oder GLP-1 Analoga intensiviert, 3.079 (4,4\%) wechselten innerhalb von 2,9 Jahren auf eine BOT/BOT plus-Therapie und
$7.834(11,3 \%)$ wechselten innerhalb von 2,6 Jahren auf eine sonstige Insulintherapie. Mit einem medianen Alter von 58,1 Jahren waren die Patienten in der Metformin-Monotherapie Gruppe am ältesten, wobei die Diabetesdauer in dieser Gruppe am kürzesten war (Median 4,6 Jahre). Patienten mit BOT oder sonstiger Insulintherapie waren jünger $(54,4$ und 52,0 Jahre) und hatten eine längere Diabetesdauer ( 9,7 und 12,6 Jahre). Patienten mit Metformin-Monotherapie hatten einen medianen $\mathrm{HbA} 1 \mathrm{c}$ von $6,6 \%$ und Patienten mit sonstiger Insulintherapie einen Hba1c von $7,7 \%$ (OAD/GLP-1-Gruppe 7,0\%, BOT-Gruppe $7,4 \%$ ). Der Anteil an Patienten mit $\geq 1$ schweren Hypoglykämie reichte von $0.3 \%$ in OAD/GLP-1-Gruppe bis $3.6 \%$ in der sonstigen Insulintherapiegruppe.

Schlussfolgerung: Die Ergebnisse der Diabetesregister DPV und DIVE deuten darauf hin, dass eine Therapieintensivierung mit Insulintherapie erst im späteren Verlauf des Typ-2-Diabetes erfolgt. Unterschiede in den Therapiegruppen wurden in Bezug auf das Alter, Diabetesdauer, der Stoffwechseleinstellung ( $\left.\mathrm{HbA}_{1} \mathrm{c}\right)$ sowie schweren Hypoglykämien beobachtet.

\section{Funding: Sanofi-Aventis}

\section{PS064}

Der Effekt von Aktivin A und Follistatin auf die Interaktion von Endothelzellen und synovialer Fibroblasten von Patienten mit rheumatoider Arthritis

Iris Aykara'; Magnus Diller'; Stefan Rehart' ${ }^{2}$; Ulf Müller- Ladner'; Elena Neumann ${ }^{1}$

${ }^{1}$ Kerckhoff Klinik Bad Nauheim, Justus-Liebig-Universität Giessen, Rheumatologie und klinische Immunologie, Bad Nauheim, Germany; ${ }^{2} \mathrm{~A}-$ GAPLESION Markus-Krankenhaus Frankfurt, Abteilung für Unfallchirurgie, Frankfurt, Germany

Der autoregulatorische Regelkreis des Proteohormons Aktivin A und dessen Antagonisten Follistatin ist bereits aus dem hypothalamisch-hgypophysären Hormonkreislauf bekannt. Allerdings spielen Aktivine auch eine Rolle bei Autoimmunerkrankungen wie der rheumatoiden Arthritis (RA). Durch Entzündungsprozesse wird Aktivin A vermehrt ausgeschüttet, welches wiederrum seinen Antagonisten Follistatin induziert. Dieser negative Feedback-Mechanismus wurde bereits bei Hepatozyten beschrieben, ist jedoch in synovialen Fibroblasten (SF) von Patienten mit RA (RASF) ausgeschaltet. SF sind für die Knorpeldestruktion bei der RA verantwortlich. Allerdings findet vor allem im entzündeten Synovium eine verstärkte Neoangiogenese statt, bei der die Interaktion der RASF mit Endothelzellen eine zentrale Rolle spielt. Der Einfluss von Aktivin und Follistatin auf die Interkation von Endothelzellen und RASF ist noch unklar.Ziel der Studie war, den Effekt von Aktivin A und Follistatin auf die Interaktion von Endothelzellen und RASF zu charakterisieren. RASF wurden aus Synovialgewebe von Patienten mit RA im Rahmen einer Gelenksersatzoperation isoliert. Die Endothelzellen (HUVEC) wurden kommerziell erworben. Die Zellen wurden anschließend in Monokultur bzw. in direkter Kokultur(1:1) mit Aktivin A(15ng/ml), Follistatin(50ong/ml), IL-1ß(1ng/ml) bzw. der Kontrolle stimuliert. Nach $18 \mathrm{~h}$ wurden die Überstände abgenommen und die Konzentration von Aktivin A, Follistatin, VEGF und IL-6 mittels ELISA gemessen. IL-1 $\beta$ induzierte die Aktivin A Ausschüttung in RASF sowie HUVECs in Monokultur sowie in direkter Kokultur. Die gleichzeitige Stimulation mit IL-1 $\beta$ und Follistatin reduzierte sowohl in HUVECs als auch in der direkten Kokultur mit RASF die Aktivin A Konzentration im Vergleich zur Stimulation nur mit IL-1 $\beta(p<0,05, n=4)$. In Monokultur der RASF konnte diese Reduktion nicht beobachtet werden. Bei den HUVECs zeigte sich eine signifikante Reduktion $(p<$ $0,05)$ der Ausschüttung des proinflammatorischen Zytokins IL-6 nach Stimulation mit Aktivin A und IL-1 $\beta$ im Gegensatz zu der alleinigen Sti- 
mulation mit IL-1 $\beta$. Bei den RASF resultierte die zusätzliche Inkubation mit Aktivin A in einem Anstieg des IL- 6 Spiegels im Vergleich zu IL-1 $\beta$, während sich in Kokultur die IL-6 Konzentration nicht veränderte.Die Ausschüttung von VEGF, welches eine wichtige Rolle bei der inflammatorisch induzierten Neoangiogenese spielt, wurde in RASF durch IL-1 $\beta(89 \%)$, Aktivin A/ IL-1 $\beta(148 \%)$ und Follistatin/ IL-1 $\beta(84 \%)$ gesteigert. In der direkten Kokultur mit HUVECs war dieser Anstieg zwar sichtbar, jedoch geringer ausgeprägt IL-1 $\beta(75 \%)$, Aktivin A/ IL-1 $\beta(101 \%)$ und Follistatin/ IL-1 $\beta(67 \%)$.Durch die Interaktion von Endothelzellen und RASF wird die proinflammatorische Antwort der RASF durch Aktivin $A$ abgeschwächt, obwohl in Monokultur eine Steigerung dieser Reaktion zu beobachten ist. Der autoregulatorische Regelkreis ist in Endothelzellen aktiv, aber nicht in RASF. In der Kokultur scheint dieser Regelkreis aktiv zu sein.

[1] de Jong FH, Grootenhuis AJ, Klaij IA, Van Beurden WM: Inhibin and related proteins: localization, regulation, and effects. Adv Exp Med Biol 1990;274:271-293.

[2] de Kretser DM, O'Hehir RE, Hardy CL, Hedger MP: The roles of activin $A$ and its binding protein, follistatin, in inflammation and tissue repair. Mol Cell Endocrinol 2012;359:101-106

\section{PS065 \\ Übergewicht als Einflussparameter der rheumatoiden Arthritis und Osteoarthritis (Arthrose) im Mausmodell}

Hani Manfred Sauermilch; Marie-Lisa Hülser'; Carina Schreiyäck'; Yubin Luo $^{2}$; Aline Bozec ${ }^{2}$; Georg Schett ${ }^{2}$; Ulf Müller-Ladner'; Elena Neumann ${ }^{1}$

\section{Gießen, Germany;}

${ }^{1}$ Campus Kerckhoff, Justus-Liebig-Universität Gießen, Klinische Rheumatologie und Immunologie, Gießen, Germany; ${ }^{2}$ Universität Erlangen-Nürnberg, Klinische Medizin ${ }^{3}$ - Immunologie und Rheumatologie, Erlangen, Germany

Hintergrund: Adipozytokine sind bioaktive Substanzen, die überwiegend vom Fettgewebe synthetisiert und sezerniert werden. Diese Botenstoffe wirken einerseits auf verschiedene Stoffwechselvorgänge und können andererseits auch auf immunologische Prozesse Einfluss nehmen.

Ziele: In dieser Arbeit wurde der Einfluss von Übergewicht auf Modellsysteme der rheumatoiden Arthritis (RA) und Osteoarthritis (OA) zu verschiedenen Krankheitszeitpunkten sowie hinsichtlich Entzündungsparametern und Adipokinen untersucht.

Methoden: Im OA Modell („DMM, Destabilisierung medialer Menisken“) wurden C57BL/6 Mäuse, im RA Modell ("CIA, Kollagen induzierte Arthritis, $\left.{ }^{\prime}\right)$ DBA/1Rj Mäuse mit einer Hochfettdiät (HFD) oder einer normalen Diät (ND) gefüttert. Blut, Fettgewebe, Organe und Gelenke wurden nach 4, 6, 8 Wochen im DMM und 4, 5,5, 7 Wochen im CIA Modell nach Induktion der OA und RA entnommen. Systemische Adiponektin-, Leptin-, und Visfatin sowie CRP Konzentrationen wurden bestimmt. Der Effekt der HFD wurde anhand eines Fettleber-Scores und der "crown- like structures (CLS)" in Fettgewebe bestimmt. Zur Beurteilung der Pathogenese dienten histologische und klinische Scores.

Ergebnisse: Die Kombination des CIA und DMM Modells mit der Adipositas-Induktion durch HFD konnte erfolgreich durchgeführt werden. Als Parameter dienten der klinische und histologische Nachweis der Gelenksdestruktionen bei DMM/CIA sowie das erhöhte Körpergewicht und die erhöhten Fettleber-Scores bei den HFD Tieren. Die CRP Konzentration wurde signifikant durch Induktion der CIA erhöht. Im DMM-Modell war die Anzahl der CLS unter HFD $(0.2 \pm 0.16, n=7)$ signi- fikant höher als unter ND (5.2 $\pm 0.98, n=8)$. In der ClA hingegen, waren die CLS der erkrankten Tiere (ND) im Vergleich zu den gesunden Tieren (ND) signifikant erhöht. Die Serum Leptin Spiegel wurden durch die HFD signifikant erhöht, dieser Effekt wurde durch die DMM und CIA Induktion reduziert. Interessanterweise reduzierte die CIA Induktion auch bei HFD Tieren die Leptin Konzentration signifikant.

Zusammenfassung: Unsere Ergebnisse zeigen, dass Übergewicht die Pathogenese der OA verstärkt. Interessanterweise lässt sich der Einfluss der HFD auf die Induktion der CIA vs. ND nicht auf die RA übertragen. Die systemischen Leptin-Konzentrationen wurden durch die CIA im Vergleich zu DMM erheblich stärker reduziert. Ebenfalls zeigten sich bei den CIA-Tieren unter ND vermehrt CLS. Anhand dieser Ergebnisse lässt sich, im Gegensatz zum Arthrosemodell, bei der entzündlichen Arthritis des CIA-Modells ein vom Körpergewicht entkoppelter, entzündlicher Mechanismus vermuten.

\section{PS066}

Können standardmäßig erhobene Laborparamter ein Comprehensive Geriatric Assessment ergänzen? - Ein Vergleich der metabolischen Signatur von älteren, multimorbiden Patienten

Lena Pickert ${ }^{1}$; Anna Maria Meyer ${ }^{2}$; Ingrid Becker ${ }^{3}$; Paul Brinkkötter ${ }^{5}$; Volker Burst ${ }^{5}$; Marietta Christiansen ${ }^{2}$; Annika Hees ${ }^{2}$; Franziska Müller ${ }^{2}$; Nicolas Noetzel $^{2}$; Marcel Rarek ${ }^{2}$; Alberto Pilotto ${ }^{4}$; Thomas Benzing ${ }^{5}$; Maria Cristina Polidori ${ }^{5}$

'Uniklinikum Köln, Klinik für Innere Medizin II, Köln, Germany; ${ }^{2}$ Universitätsklinikum Köln, Klinik II für Innere Medizin, Schwerpunkt Klinische Altersforschung, Köln, Germany; ${ }^{3}$ Universität zu Köln, Institut für Medizinische Statistik und Bioinformatik, Köln, Germany, ${ }^{4}$ Galliera Hospital, Department of Orthogeriatrics, Rehabilitation and Stabilization, Genova, Italy; ${ }^{5}$ Universitätsklinikum Köln, Klinik II für Innere Medizin, Nephrologie, Rheumatologie, Diabetologie und Innere Medizin, Köln, Germany

Zeit ist in Krankenhäusern ein kostbares Gut. Die Antwort auf den Druck, den steigende Patientenzahlen und die damit gewachsenen Anforderungen auf die medizinische Versorgung ausüben, ist zeiteffizientes Arbeiten. Um Hochrisikopatienten, wie multimorbide, ältere Patienten, schnell zu identifizieren, gilt das Comprehensive Geriatric Assessment (CGA) als Goldstandard. Ein relativ neues Forschungsgebiet stellt die Frage nach einer metabolischen Signatur und deren prognostische Aussagekraft bei oben genannten Patienten dar. Ob es möglich ist, durch Laborparameter als metabolische Signatur die Ergebnisse einer CGA-basierenden Prognoseberechnung zu ergänzen, ist Gegenstand der vorliegenden Untersuchung.

Retrospektiv wurden aus einer bestehenden Database zwei Gruppen von insgesamt 199 multimorbiden Patienten > 70 Jahre verglichen, die auf der nephrologischen Akutstation der Klinik II für Innere Medizin des Universitätsklinikums Köln hospitalisiert waren. Eine Gruppe hatte eine nephrologische, die andere Gruppe eine andere Aufnahmediagnose (hauptsächlich kardiovasuklär oder endokrinologisch). Alle Patienten erhielten bei Aufnahme ein CGA und eine Prognoseberechnung mittels des Multidimensionalen Prognostischen Index (MPI). Berücksichtigt wurden dabei die funktionellen, körperlichen, sozialen und geistigen Aspekte der Patienten. Die Ergebnisse des MPI ergeben kontinuierliche Werte zwischen o und 1, die zu drei Risikogruppen für Rehospitalisierung, Mortalität und Institutionalisierung zusammengefasst werden: $0.00-0.33$ geringes, $0.34-0.66$ mittleres und $0.67-1.00$ hohes Risiko. Untersucht wurden die Elekrolytwerte, nephrologische Retentionsparamter, Werte für das blutbildende System und der Vitaminstatus der Patienten.

97 Patienten $(64 \mathrm{M}, 33 \mathrm{~F})$ hatten eine nephrologische Aufnahmediagnose, 102 Patienten $(65 \mathrm{M}, 37 \mathrm{~F})$ eine anderen Aufnahmediagnose. Das Durchschnittsalter betrug 78,4 +/- 5,5 (nephrologisch) und 79,2 +/- 5,3 
Jahre (andere), die durchschnittliche Länge der Hospitalisierung 15,2 (nephrologisch) und 14,8 Tage (andere). Bei Patienten mit nephrologischer Aufnahmediagnose gab es eine signifikante Korrelation $(p<$ $0,05)$ zwischen den Albumin-Werten bei Aufnahme und der Zuteilung in Risikogruppen des MPI. Bei Patienten ohne nephrologische Aufnahmediagnose gab es eine signifikante Korrelation $(p<0,05)$ zwischen den Vitamin-D Werten bei Aufnahme und der Zuteilung in Risikogruppen des MPI.

Die vorliegenden Ergebnisse zeigen, dass es möglich ist, mithilfe einer metabolischen Signatur die prognostische Aussagekraft des MPI zu ergänzen. Die oben genannten Ergebnisse stehen in einer Linie mit einer bereits veröffentlichen Studie. Eine tiefergehende Analyse der Daten wird durchgeführt, um eine mögliche Beeinflussung der Ergebnisse auszuschließen und um zusätzliche relevante Parameter zu detektieren. Weiterführende Forschung ist notwendig, um den genauen Einfluss der Laborparameter auf die Aussagekraft einer CGA-basierenden Prognoseberechnung zu identifizieren.

\section{PS067 \\ Administrative Koordination vernetzter translationaler Forschung am Beispiel des Deutschen Konsortiums für Translationale Krebsforschung (DKTK)}

Claus Zippel'; Karina Förster²; Jürgen Frank ${ }^{3}$; Franziska Hasslinger-Pajtler ${ }^{4}$; Anja Hernandez ${ }^{5}$; Sabine Kaull ${ }^{6}$; Christine Koppenhöhl7; Julia Serr ${ }^{8}$; Anette Reil-Held ${ }^{9}$

'Deutsches Krebsforschungszentrum (DKFZ), Deutsches Konsortium für Translationale Krebsforschung (DKTK), Heidelberg, Germany; ${ }^{2}$ Deutsches Krebsforschungszentrum (DKFZ), Deutsches Konsortium für Translationale Krebsforschung (DKTK), Dresden, Germany; ${ }^{3}$ Deutsches Krebsforschungszentrum (DKFZ), Deutsches Konsortium für Translationale Krebsforschung (DKTK), Tübingen, Germany; ${ }^{4}$ Deutsches Krebsforschungszentrum (DKFZ), Deutsches Konsortium für Translationale Krebsforschung (DKTK), Frankfurt/Mainz, Germany; ${ }^{5}$ Deutsches Krebsforschungszentrum (DKFZ), Deutsches Konsortium für Translationale Krebsforschung (DKTK), Freiburg, Germany; ${ }^{6}$ Deutsches Krebsforschungszentrum (DKFZ), Deutsches Konsortium für Translationale Krebsforschung (DKTK), Essen/Düsseldorf, Germany; ${ }^{7}$ Deutsches Krebsforschungszentrum (DKFZ), Deutsches Konsortium für Translationale Krebsforschung (DKTK), München, Germany; ${ }^{8}$ Deutsches Krebsforschungszentrum (DKFZ), Deutsches Konsortium für Translationale Krebsforschung (DKTK), Berlin, Germany; ${ }^{9}$ Deutsches Krebsforschungszentrum (DKFZ), Deutsches Konsortium für Translationale Krebsforschung (DKTK), Heidelberg, Germany

Einleitung: Neue, vielversprechende Ergebnisse aus der Krebsforschung sollen möglichst schnell in die klinische Entwicklung und Anwendung gelangen. Zur Unterstützung dieses Ziels wurde 2012 das Deutsche Konsortium für Translationale Krebsforschung (DKTK) als eines der sechs Deutschen Zentren der Gesundheitsforschung gegründet $[1,2]$. Mit dem DKFZ als Kernzentrum kooperieren im DKTK in der Krebsforschung ausgewiesene Universitätskliniken und Forschungseinrichtungen an bundesweit sieben Standorten. Die Bündelung von Kräften in einem solchen Konsortium ist insb. mit Blick auf die Herausforderungen der personalisierten Onkologie erforderlich, wozu auch gemeinsame Plattformen beitragen. Eine erfolgreiche standort-/disziplinübergreifende Zusammenarbeit verlangt neben einer eng abgestimmten wissenschaftlichen Strategie auch die Koordination einer Vielzahl von administrativen Fragen und Vorgängen zwischen den Partnern. Wir stellen die administrative Ebene des DKTK dar und zeigen, welche Vorteile sich für die administrative Begleitung standortübergreifend translationaler Forschung durch die aufgebauten Strukturen bieten.
Ergebnis: Mit dem DKTK wurde eine Organisationsstruktur etabliert, über die an mehreren Zentren geplante Forschungsvorhaben/IITs aus administrativer Sicht unter wiederkehrenden Bedingungen und damit relativ effektiv und effizient koordiniert werden können. Bei der Administration des DKTK kommt den administrativen Koordinatoren eine wichtige Rolle zu, die an den Standorten die Schnittstelle zwischen standortübergreifend geltenden DKTK-Prozessen einerseits und den standortspezifischen administrativen Abläufen andererseits bedienen. Sie stehen in engem Austausch mit dem Vorstand und den administrativen Abteilungen des DKFZ Kernzentrums und fungieren gleichzeitig als Ansprechpartner für die lokalen DKTK-Wissenschaftler, etwa bei Budgetfragen oder Beschaffungen. Erfolgsfaktoren sind u.a. bestehende Rahmenvereinbarungen, ein standardisiertes Projektmanagement und Größeneffekte. Seit DKTK-Gründung haben verschiedene (interdisziplinäre) Forscherteams die DKTK-Struktur genutzt, um Projekte unter Beteiligung mehrerer Standorte/Prüfzentren administrativ aufzusetzen und zu koordinieren. Ein Beispiel dafür ist die PSMA-Studie zur Evaluierung eines neuen PET-Tracers für die Prostatakrebsdiagnostik [3].

Diskussion: Die Durchführung einrichtungsübergreifender translationaler Forschungsvorhaben wird in der Krebsforschung insb. aufgrund der Entwicklungen in der personalisierten Onkologie und dem großen Datenbedarf für den Einsatz künstlicher Intelligenz immer wichtiger. Hierzu bildet auch die administrative Koordination angesichts vieler Partner aus Klinik, Forschung und Verwaltung einen Erfolgsfaktor. Mit dem DKTK wurde eine bundesweite Struktur etabliert, über die translationale Forschung an mehreren Zentren gezielt administrativ unterstützt werden kann. Insb. bei multizentrischen IITs führt dies zu Zeitersparnis und besserer Wissens-/Ressourcennutzung.

[1] Hasenfuß G. Deutsche Zentren der Gesundheitsforschung Schneller von der Idee zum Patienten. Dtsch med Wochenschr 2014; 139:2487-88

[2] Joos $\mathrm{S}$ et al. German Cancer Consortium (DKTK) - A national consortium for translational cancer research, Mol Oncol. 2018; doi: 10.1002/1878-0261.12430

[3] Zippel C et al. Administration klinischer Studien in der Nuklearmedizin: Organisationale Herausforderungen und Anregungen für Studienleiter und -koordinatoren am Beispiel des DKTK-PSMA-Multicenter Trials. Der Nuklearmediziner 2018; 41: 264-270

\section{PS068 \\ Diagnostic exome sequencing in cardiac diseases - a single center experience}

Anastasia Gazou; Miriam Stampfer; Andreas Dufke; Olaf Rieß; Tobias Haack; Stephan Waldmüller

Universitätsklinikum Tübingen, Institut für Medizinische Genetik und Angewandte Genomik, Tübingen, Germany

Introduction: Genetic causes should be considered in both syndromic and non-syndromic forms of cardiac diseases. Monogenic cardiac diseases have a prevalence of 1:20000 and represent a major socioeconomic burden. Identification of the underlying genetic defect remains challenging due to the vast heterogeneity and mostly unspecific clinical features. However, establishing a firm molecular diagnosis is important for risk stratification of affected individuals and their families. Information on genotype-phenotype correlations might guide monitoring for known accompanying clinical features. For instance it is known that patients with RBM20-associated cardiomyopathy bare a higher arrhythmia risk than patient with TTN-associated disease. 
Methods: During the past two years we used exome-sequencing (enrichment Agilent Human Sure Select All exon kit v5/ v6/ v7, sequencing Illumina HiSeq 2500/ Novaseq 6000) to investigate 74 index patients with suspected genetic cardiomyopathies or primary cardiac arrhythmias. Using an in-house bioinformatics pipeline we prioritized rare variants in known disease-associated genes as well as in genes associated with clinical signs of differential diagnosis (including syndromic conditions).

Results: In approximately $32 \%$ of cases ( 24 out of 74 patients) we established a firm molecular diagnosis. In about 19\% of cases (14 out of 74 patients) we identified variants of unknown significance in known or putative disease-associated genes. Detection rate was $23.9 \%$ (20 out of 58 patients) in cardiomyopathy patients and $25 \%$ (4 out 16 patients) in patients with suspected primary arrhythmias. Highest detection rates were observed in $\mathrm{NCM}, \mathrm{LQTS}, \mathrm{H}(\mathrm{O}) \mathrm{CM}$ and ARVC patients with detection rates of $66 \%$ ( 2 out of 3 patients), $40 \%$ ( 4 out of 10 patients), $44 \%$ ( 8 out of 18 patients) and $44 \%$ ( 4 out of 9 patients) respectively.

Conclusion: Exome sequencing allows the identification of likely genetic cause of cardiac conditions in a substantial fraction of patients. The identification of the molecular bases of diseases allows risk stratification in patients and might guide treatment decisions (e.g. ICD-implantation). It also enables (predictive) genetic testing for relatives and thus an early identification of persons at risk for developing cardiac incidents. On the other hand it allows "discharge" of relatives from intensified cardiologic surveillance when a disease-causing mutation can be excluded. Thus genetic testing via exome sequencing is a crucial component of an interdisciplinary approach to the management of patients with primary cardiomyopathies and arrhythmias.

\section{PS069 \\ Assessment of gastrointestinal symptoms in patients with atrial fibrillation undergoing pulmonary vein isolation}

Richard Pfrogner'; Valérie Pavlicek'; Dominic Millenaar'; Amjad Razouk²; Michael Böhm²; Christian Ukena

'Universitätsklinikum Homburg, Innere Medizin III, Klinik für Kardiologie, Angiologie und Internistische Intensivmedizin, Homburg, Germany; ${ }^{2}$ Uniklinikum Homburg, Innere Medizin III, Klinik für Kardiologie, Angiologie und Internistische Intensivmedizin, Homburg, Germany

Background: Atrial fibrillation (AF) is the most common cardiac arrhythmia. Similar autonomic in-nervation of the esophagus and left atrium with sympathovagal imbalance may contribute to the relation between non-valvular atrial fibrillation (AF) and gastroesophageal reflux disease (GERD).

Purpose: The aim of our prospective study was to evaluate gastrointestinal symptoms in patients with AF undergoing pulmonary vein isolation (PVI). Furthermore, we investigated the effects of PVI on these symptoms during follow up.

Methods: Gastrointestinal symptoms were assessed by the standardized and validated PAGI SYM (Patient Assessment of Gastrointestinal Symptom Severity Index) questionnaire before scheduled PVI and six months after PVI. The PAGI SYM is intended to cover the main symptom groupings over a 2-week recall period for GERD, dyspepsia and gastroparesis.

Results: In 82 patients with symptomatic AF before PVI (age $62 \pm 10$ years, $73 \%$ male, BMI $29 \pm 5 \mathrm{~kg} / \mathrm{m} 238 \%$ EHRA III-IV, LVEF $55 \pm 9 \%$ ), $27 \%$ were suffering from GERD treated with a proton pump inhibitors (PPI). In terms of paroxysmal versus persistent $\mathrm{AF}$, there was no significant difference regarding PPI medication $(p=0.98)$. In the total population, the PAGI SYM median total score was 0.5 (0-3) corresponding to very mild gastrointestinal symptoms. In detail, $49 \%$ of patients showed no GI disorders, $43 \%$ mild Gl symptoms and only 5\% suffered from moderate gastrointestinal disorders. There were no patients with severe Gl symptoms observed. Furthermore, there were no associations between intensity of $\mathrm{Gl}$ symptoms, and gender, type of AF, left atrial size. There was a significant association between the PAGI SYM total score and the EHRA class: A higher EHRA score correlated with more UGI-Symptoms $(r=0.249, p=0.027)$. No significant changes were observed within the PAGI-SYM-Score before and after PVI. $(p=0.68)$

Conclusions: Half of the patients undergoing PVI due to symptomatic AF showed no Gl symptoms, the other half only mild disorders. A correlation between AF symptoms and Gl symptoms were observed which may indicate an interaction between the heart and the gut in these patients.

\section{PS070}

Diabetisches Fußsyndrom:

Bewertung des Ausbildungsstands im Medizinstudium

\section{Anna Mavromanoli'; Areti Katsouda ${ }^{2}$; Dimitrios Karamanos ${ }^{3}$}

'Aristoteles-Universität Thessaloniki, Physik, Aristoteles-Universität Thessaloniki, Griechenland, Greece; ${ }^{2}$ Allgemeines Krankenhaus der Stadt Lamia, Griechenland, Allgemeine Chirurgie, Allgemeines Krankenhaus der Stadt Lamia, Griechenland, Greece; ${ }^{3}$ Papageorgiou Universitätsklinik und Aristotäles Universität Thessaloniki, Allgemeine Chirurgie, Papageorgiou Universitätsklinik, Aristoteles-Universität Thessaloniki, Griechenland, Greece

Zweck: Die Bewertung des Wissens von Medizinstudenten in Bezug auf die Diagnose und Behandlung des diabetischen Fußsyndroms.

Methode: In dieser Studie sind Studierende der medizinischen Fakultät der Aristoteles-Universität Thessaloniki, Griechenland eingeschlossen worden. Die Teilnehmer haben im Jahr 2016 freiwillig einen anonymen, über das Internet hergestellten Fragebogen bezüglich des Diabetes mellitus und der Komplikation des diabetischen Fußsyndroms ausgefüllt. Die Antworten auf den Fragenbogen wurden mit dem statistischen Programm SPSS 22.0 verarbeitet.

Ergebnisse: Die statistische Analyse hat gezeigt, dass die Teilnehmer über ein gutes Wissen für die Symptome von Diabetes mellitus und seiner möglichen Komplikationen verfügen. Fast die Hälfte der Beantworter (55\%) erkennt die Notwendigkeit für die tägliche Kontrolle, Reinigung und Pflege der Füße bei Patienten mit Diabetes mellitus, aber es zeigt sich, dass das Bewusstsein über mehr spezifische Anweisungen für die Behandlung der Patienten mit diabetischen Fußsyndrom häufig fehlt. Die Teilnehmer würden mehr Aufmerksamkeit auf Veränderungen der Haut und der Morphologie des Fußes ziehen (92\%), aber die Veränderung der Temperatur und des Geruchs bringen sie in Verwirrung bezüglich der Notwendigkeit der Situation oder die Überweisung an Spezialisten (64\% wegen Veränderung der Temperatur, 49\% wegen Veränderung des Geruchs).

Schlussfolgerungen: Es scheint, dass trotz des zufriedenstellenden Bewusstseins für Diabetes mellitus im Medizinstudium, ist es notwendig, die Möglichkeit für die Entwicklung der Komplikation des diabetischen Fußsyndroms bei Patienten mit Diabetes mellitus in der medizinischen Ausbildung hervorzuheben, sodass die Absolventen in der Lage sind, diese Komplikation zu diagnostizieren, zu behandeln und, wenn notwendig, die Anforderung an eine Überweisung an Spezialisten zu erkennen. 


\section{PS071 \\ Using logical memory as a reliable 'cognitive vital sign' to monitor cognition in hospital}

Thomas Mross'; William David Molloy'; Ronan O Caoimh ${ }^{3}$; Afsana Habib ${ }^{4}$; Roger Clarnette ; Padraic Nicholas ${ }^{2}$; Yang Gao ${ }^{2}$

${ }^{1}$ Agaplesion Bethanien Krankenhaus Heidelberg gemeinnützige $\mathrm{GmbH}$, Geriatrie, Heidelberg, Germany; ${ }^{2}$ Mercy University Hospital Cork, Geriatrics, Cork, Ireland; ${ }^{3}$ Galway University, Geriatrics, Galway, Ireland; ${ }^{4}$ School of Medicine and Pharmacology, University of Western Australia, Geriatrics, Perth, Australia

Background: Although, there is a high prevalence of delirium and cognitive impairment among hospitalised older adults, short, reliable cognitive measures are not available to monitor cognitive function and potentially alert healthcare professionals to early changes.

Aim: We evaluated the reliability, responsiveness and feasibility of logical memory, immediate verbal recall of a short story, compared to brief tests of attention as a bedside "Cognitive Vital Sign" in order to improve time to initialize treatment and therefore improve patient outcomes.

Methods and patient characteristics: Out of 100 recruited patients, trained nursing staff performed twice-daily cognitive assessments on 84 clinically stable inpatients, 47 Males and 37 Females, in geriatric units in two hospitals over 3-5 consecutive days using logical memory and short tests of attention including months-of-the-year backwards, numbers counting forwards, numbers counting backwards and orientation to time and digit span. The majority of patients were aged 65 and above $\mathrm{N}=69$.

Twelve patients were excluded due to being discharged less than three days after commencing the study. Four patients clinical condition worstened during the participation in the study and further measurements were not possible.

Logical memory was compared to these various short teests of attention. Ten different alternative logical memory stories were developed in order to reduce learning effect.

Results: Scores were compared to those of an expert rater. Inter-rater reliability was excellent with correlation coefficients for logical memory increasing from $r=0.87$ on day one to $r=0.97$ by the fourt day ( $p$ $<0.0001)$.

Logical memory scores were statistically similar $(p=0.99)$ with repeated testing and no learning effect could occur due to ten variations of the logical memory test.

Mean logical memory scores ranged from o to 30 . The mean score was 10.7/30. The mean variations in scores was 3.4 points with standard deviation +-1.1 points, indicating that fluctuations of three points or greater from the individuals baseline were outside normal daily changes and therefore clinically significant.

Patients who had been listening to the news, had breakfast or recently checked the time in anticipation of cognitive testing scored better which reflects real-life practice; a strength of the study.

All fourteen nurses taking part in the study reported that logical memory was feasible to score routinely and were willing to perform the logical memory along other vital signs in the future.

Conclusion: Logical memory is a reliable measure of cognition showing diurnal variation but no evident learning effects. Further study is required to define the properties of an ideal cognitive vital sign, although logical memory may satisfy these objectives.

\section{PS072 \\ Ernährungsstatus und Körperzusammensetzung von Patienten mit Glioblastomen}

Dejan Reljic'; Joachim Herrmann'; Markus Neurath²; Yurdagül Zopf ${ }^{3}$

${ }^{1}$ Hector Center für Ernährung, Bewegung und Sport, Medizinische Klinik ${ }^{1}$, Ulmenweg ${ }^{18}$, Germany; ${ }^{2}$ University Erlangen, Medizinische Klinik ${ }^{1},{ }^{91054}$, Germany; ${ }^{3}$ Universitätsklinikum Erlangen - Medizinische Klinik ${ }^{1}$, Hector-Center für Ernährung, Bewegung und Sport, Erlangen, Germany

Hintergrund: Patienten mit einer Krebserkrankung haben ein erhöhtes Risiko für eine Mangelernährung. Tumorerkrankungen führen zu einer systemischen Inflammation und zu einer verstärkten Zytokinexpression, die nachweislich verschiedene Hormon- und Stoffwechselprozesse aktivieren können. Die resultierende Tumorkachexie führt insbesondere zum progressiven Muskelverlust. Zudem kann die tumorspezifische Therapie mit Chemo- und Strahlentherapie aufgrund von Schädigungen der Darmschleimhaut und gastrointestinalen Nebenwirkungen eine Malabsorption und eine weitere Verschlechterung der Nährstoffaufnahme zur Folge haben. Vor allem bei den gastrointestinalen Tumoren (GI) sind diese Veränderungen der Körperzusammensetzung sehr gut beschrieben. Es gibt jedoch kaum Daten über den Ernährungszustand und der Körperzusammensetzung bei Glioblastompatienten (GBP).

Methode: Über einen Zeitraum von 2 Jahren wurden im Rahmen einer Querschnittsstudie $100 \mathrm{~GB}$ in Bezug auf ihre Nährstoffaufnahme, Körperzusammensetzung und muskuläre Kraft untersucht. Die Ernährung wurde mittels eines standardisierten Ernährungsfragenbogens erfasst und analysiert, die Körperzusammensetzung mittels bioelektrischer Impedanzanalyse untersucht und die isometrische Handmuskelkraft mittels eines hydraulischen Handkraft-Dynamometers bestimmt. Die Ergebnisse wurden mit den Messdaten von Patienten mit Gl-Tumoren verglichen.

Ergebnisse: Es zeigen sich bei den GBP korrelierend zu den publizierten Daten von Patienten mit GI Tumoren reduzierte Muskelmassen. Das Gewicht betrug 79,8 $\pm 18,1 \mathrm{Kg}$, der Phasenwinkel 4,9 $\pm 0,78$ und die Skelettmasse $25 \pm 7,1 \mathrm{Kg}$. Die Kalorienaufnahme betrug $1622 \mathrm{kcal} \pm 479$. Die Proteinaufnahme betrug $0,43 \pm 0,53 \mathrm{~g} / \mathrm{Kg}$, die Fettaufnahme $0,40 \pm 0,5 \mathrm{~g} / \mathrm{Kg}$ und Kohlenhydrate $1,03 \pm 1,24 \mathrm{~g} / \mathrm{Kg}$. Die max. Handkraft betrug $37,7 \pm 12,7$. Sowohl das Gewicht $(74,6 \pm 16,9 \mathrm{Kg})$, der Phasenwinkel $(4,5 \pm 0,79)$ und die Sklelettmuskelmasse $(23,8 \pm 6,94 \mathrm{~g} / \mathrm{Kg})$ waren bei den GI Tumoren signifikant niedriger. Die Kalorienaufnahme (2090 kcal \pm 672$)$ und die Aufnahme der Makronährstoffe waren bei den GI Tumoren signifikant höher (Proteine 1,34 $\pm 0,68 \mathrm{~g} / \mathrm{Kg}$; Fett

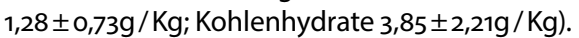

Zusammenfassung: Patienten mit GI Tumoren haben nachweislich aufgrund von Störungen im Gl Trakt eine Ernährungsstörung mit reduzierter Muskel- und Fettmasse. Auch wenn Patienten mit einer GBP weniger GI Störungen vorweisen, liegen ebenfalls reduzierte Muskelmassen vor. Die Kalorienaufnahme und die Aufnahme der Makronährstoffe und insbesondere der Kohlenhydrate waren signifikant niedriger. Diese ist wahrscheinlich durch die ketogene Diät bedingt, die bei den GBP durchgeführt wird. Auch wenn die inflammativen Prozesse bei den GI Tumoren zu einer höheren Muskelmassenabnahme führen, kommt es aufgrund der reduzierten Nährstoffaufnahme und dem Tumorprozess ebenfalls zu einer Abnahme des Sklettmuskelmasse. Auch bei GBP sollte frühzeitig eine Erfassung des Ernährungsstatus und der Körperzusammensetzung durchgeführt werden. 


\section{Internistische Intensivmedizin}

\section{PS073}

The gut incretin hormone GIP inhibits inflammatory activation of vascular smooth muscle cells

Robert Werner Mertens'; Florian Kahles'; Irmgard Diepolder'; Constantin Halim'; Paul Idel'; Julia Möllmann'; Sebastian Diebold'; Hannes Michael Findeisen ${ }^{2}$; Corinna Lebherz; ; Nikolaus Marx'; Michael Lehrke ${ }^{1}$

${ }^{1}$ Uniklinik RWTH Aachen, Medizinische Klinik I, Aachen, Germany; ${ }^{2}$ Uniklinikum Münster, Klinik für Kardiologie I: Koronare Herzkrankheit, Herzinsuffizienz und Angiologie, Münster, Germany

Purpose: The incretin hormones GLP-1 (glucagon-like peptide-1) and GIP (glucose-dependent insulinotropic peptide) are secreted from the gut following food intake leading to pancreatic insulin secretion and glucose lowering. GLP-1 agonism has recently been found to inhibit neointima formation after vascular injury in rodents (experimental model of in-stent restenosis) due to inhibition of vascular smooth muscle cell proliferation. In this study we analysed the effect of the other main incretin GIP on vascular remodeling.

Methods and Results: Since vascular smooth muscle cells (VSMC) play a crucial role in pathological vascular remodeling processes like atherosclerosis or in-stent restenosis, we first asked whether GIP does affect VSMC proliferation under in vivo conditions. Using an adeno-associated viral vector system we overexpressed GIP (1-42) and LacZ as

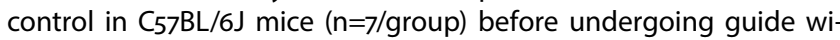
re-induced endothelial denudation injury of the left femoral artery. Histological analyses were performed 4 weeks after vascular injury and revealed no difference in neointima formation and intima/media ratio between both groups, indicating that GIP has no direct effect on VSMC proliferation under in vivo conditions. Consistent with our in vivo findings GIP incubation showed no effect on mitogen-induced VSMC proliferation analyzed by BrdU incorporation $24 \mathrm{~h}$ after PDGF$\mathrm{BB}$ stimulation under in vitro conditions. In the next step we wondered whether GIP might affect inflammatory activation of VSMC. Indeed GIP treatment significantly diminished protein and mRNA expression of the cell adhesion molecule VCAM- 1 by $50 \%$ (control $6.8 \pm$ 0.9 Vs. $3.5 \pm 1.0 \mathrm{GIP}$ fold change from basal; $p<0.05$ ) in TNF- $a$ treated VSMC. Furthermore, GIP decreased TNF- $a$-induced MMP-2 and MMP9 expression in VSMC, suggesting a potential protective role of GIP in inflammation-induced vascular remodeling.

Conclusion: Unlike GLP-1 the other main incretin hormone GIP does not affect proliferation of VSMC in vitro or in vivo. However, GIP has direct anti-inflammatory effects in TNF-a treated VSMC by diminishing expression of cell adhesion molecules and extracellular-matrix-remodeling (ECM) markers. Further studies are needed to determine whether activation of the GIP-system might be protective in inflammation-driven vascular pathologies like atherosclerosis.

\section{PS074}

Assessment of biomarkers of cell cycle arrest and soluble urokinase-type plasminogen activator receptor for the prediction of need for renal replacement therapy in sepsisinduced acute kidney injury: A prospective, observational study

Christian Nusshag '; Christoph Rupp²; Claudius Speer'; Florian Kälble'; Martin Zeier'; Markus A. Weigand ${ }^{2}$; Florian Uhle ${ }^{2}$; Uta Merle ${ }^{3}$; Christian Morath'; Thorsten Brenner ${ }^{2}$

${ }^{1}$ Universitätsklinikum Heidelberg, Nephrologie, Heidelberg, Germany; ${ }^{2}$ Universitätsklinikum Heidelberg, Anästhesiologie, Heidelberg, Germany; ${ }^{3}$ Universitätsklinikum Heidelberg, Gastroenterology, Heidelberg, Germany

Background: Sepsis-induced acute kidney injury (AKI) is the dominating AKI etiology in critically ill patients and substantially associated with risk of death [1]. In the absence of kidney-specific therapeutic alternatives, renal replacement therapy (RRT) is often the final therapeutic option. However, whether and when to start RRT is an ongoing controversy [2]. A major issue that persists is to differentiate patients with progressive AKI and need for RRT from those with autonomous renal recovery $[3,4]$. We investigated the diagnostic value of the soluble urokinase-type plasminogen activator receptor (suPAR) and the product of the two G1-cell cycle arrest and tubular injury biomarkers tissue inhibitor of metalloproteinase-2 and insulin-like growth factor-binding protein 7 ([TIMP-2 $]^{*}[$ IGFBP7]) to predict future need for RRT

Methods: In a prospective, observational trial, 100 critically ill patients were enrolled immediately after the fulfillment of Sepsis-3 criteria. SuPAR levels were measured once at inclusion, and urinary [TIMP-2]* ${ }^{*}$ IG$\mathrm{FBP} 7$ ] levels were measured over time. The primary clinical endpoint was the occurrence of need for RRT (predefined criteria) within 7 days. Area under the receiver-operating characteristic curves (AUC-ROC) and logistic regression models were calculated.

Results: 19 patients developed need for RRT. Urinary [TIMP-2] ${ }^{*}[$ IGFBP7] levels after $12 \mathrm{~h}$ and $24 \mathrm{~h}$, and suPAR levels at inclusion outperformed all routine parameters of renal impairment with AUC's of $0.83(95 \% \mathrm{Cl}$ $0.71-0.95, \mathrm{p}<0.0001), 0.89$ (0.80-0.98, p < 0.0001) and 0.83 (0.75-0.92, p $<0.0001)$, respectively. The best discrimination ability for the primary outcome measure was achieved for [TIMP-2]*[IGFBP7] after $24 \mathrm{~h}$ of sepsis therapy, applying a cut-off value of $\geq 0.60(\mathrm{ng} / \mathrm{ml}) \wedge_{2} / 1000$ (sensitivity $90.9 \%$, specificity $67.1 \%$ ). SuPAR performed best by applying a cut-off value of $\geq 8.53 \mathrm{ng} / \mathrm{mL}$ (sensitivity $84.2 \%$, specificity $82.7 \%$ ). The diagnostic value of suPAR and [TIMP-2]*[IGFBP7] persisted even in a subset of patients with moderate to severe AKI at the time of inclusion and after adjustment for confounders. In the same subset of patients, the diagnostic accuracy of surrogate parameters of renal function diminished. Of note, the combination of [TIMP-2] ${ }^{*}\left[{ }^{\prime G F B P 7}\right]$ or suPAR with Cystatin $C$ resulted in an additive improvement of diagnostic accuracy with AUC's of $0.93(0.86-1.00)$ and 0.89 (0.82-0.96), respectively.

Conclusions: [TIMP-2]*[IGFBP7] and suPAR provide an excellent and superior diagnostic accuracy for RRT prediction compared to standard parameters of kidney function. Urinary [TIMP-2]* ${ }^{[I G F B P 7] ~ l e v e l s ~}$ after $12 \mathrm{~h}-24 \mathrm{~h}$ of sepsis therapy are suggested as an excellent diagnostic tool to monitor the success of kidney-directed sepsis therapy, whereas suPAR predicts the need for RRT already at baseline. To promote the significance of both biomarkers for clinical decision making, beneficial effects on outcomes need to be demonstrated in future studies.

[1] Kellum JA, Prowle JR (2018) Paradigms of acute kidney injury in the intensive care setting. Nat Rev Nephrol 307:2265-230. doi: 10.1038/ nrneph.2017.184 
[2] Nusshag C, Weigand MA, Zeier M, et al (2017) Issues of Acute Kidney Injury Staging and Management in Sepsis and Critical Illness: A Narrative Review. Int J Mol Sci 18:1387. doi: 10.3390/ijms18071387

[3] Gaudry S, Hajage D, Schortgen F, et al (2016) Initiation Strategies for Renal-Replacement Therapy in the Intensive Care Unit. N Engl J Med 375:122-133. doi: 10.1056/NEJMoa1603017

[4] Barbar SD, Clere-Jehl R, Bourredjem A, et al (2018) Timing of Renal-Replacement Therapy in Patients with Acute Kidney Injury and Sepsis. N Engl J Med 379:1431-1442. doi: 10.1056/NEJMoa1803213

\section{PS075}

Schwerstes akutes Lungenversagen als Folge eines sekundären Goodpasture-Syndroms nach inhalativem Substanzmissbrauch

Christoph Fisser'; Thomas Müller'; Michael Pfeifer ${ }^{2}$; Christoph Lerzer ${ }^{3}$; Matthias Lubnow ${ }^{1}$

'Universitätsklinikum Regensburg, Klinik und Poliklinik für Innere Medizin II, Regensburg, Germany; ${ }^{2}$ Universitätsklinikum Regensburg, Fachklinik für Lungenerkrankungen Donaustauf, Krankenhaus der Barmherzigen Brüder Regensburg, Abteilung für Pneumologie, Regensburg, Germany; ${ }^{3}$ Fachklinik für Lungenerkrankungen Donaustauf, Abteilung für Pneumologie, Donaustauf, Germany

Der 18-jährige Elektroniker (BMI $23,1 \mathrm{~kg} / \mathrm{m}^{2}$ ) stellt sich am 13.08. mit gering ausgeprägten Hämoptysen, Schüttelfrost, subfebrilen Temperaturen und Belastungsdyspnoe in einem externen Krankenhaus vor. Eine Therapie mit Ampicillin/Sulbactam und Clarithromycin wird begonnen. Bei progredienter respiratorischer Verschlechterung und erhöhten Infektwerten (CRP $520 \mathrm{mg} / \mathrm{l}, \mathrm{n}<5 \mathrm{mg} / \mathrm{l}$ ) wird auf Meropenem und Moxifloxacin eskaliert, und eine invasive Beatmung begonnen. Aus der Vorgeschichte ist ein Nikotinabusus (0,5 packyears) zu eruieren.

Eine Therapie mit NO, lloprost und Bauchlage erbringt keine Verbesserung. Bei schwerem ARDS ( $\mathrm{PaO}_{2} / \mathrm{FiO}_{2} 79 \mathrm{mmHg}, \mathrm{PaCO}_{2} 113 \mathrm{mmHg}$, $\mathrm{pH}$ 7,19) mit hoher Beatmungsinvasivität (IPAP $35 \mathrm{cmH}_{2} \mathrm{O}$, PEEP $15 \mathrm{~cm}$ $\mathrm{H}_{2} \mathrm{O}, \mathrm{AMV} 10,4 \mathrm{l} / \mathrm{min}$ ), erfolgt die Anlage einer venovenösen extrakorporalen Membranoxygenierung (vvECMO) mit Verlegung in unser Haus. Am 20.08. lässt sich im HRCT eine Panbronchiolitis mit disseminierten zentrilobulären Knötchen sehen.

Alle externen Untersuchungen (Bronchoskopie, Blutkulturen, Sonographie inkl. TEE, Urinstatus und -sediment, ANA, ANCA, $C_{3}, C_{4}$, anti-CCP, anti-GBM, Immunglobuline, Virologie, Bakteriologie und Mykologie (u.a. Influenza, Hanta, Hepatitis, Mycoplasmen, Leptospiren, Legionellen, Chlamydien, PJP, Aspergillen) verbleiben unauffällig.

In der erneuten diagnostischen Aufarbeitung 7 Tage später zeigt sich nun der Antikörper gegen Bestandteile der glomerulären Basalmembran deutlich erhöht ( $183 \mathrm{U} / \mathrm{ml} ; \mathrm{n}<20 \mathrm{U} / \mathrm{ml})$. Die erneute quantitative und qualitative Urinuntersuchung sowie die Nierenfunktion verbleiben unauffällig.

Wir stellten die Diagnose eines sekundären ausschließlich pulmonalen Goodpasture-Syndroms.

Eine Therapie mittels täglicher Plasmapherese, hochdosiertem Prednisolon (21.08.) und Cyclophophamid (28.08.) erfolgt. Ein Spannungs-Pneumothorax wird mittels Thoraxdrainage versorgt. Es erfolgt die komplikationslose dilatative Tracheotomie, die Sedierung wird beendet. Der Patient bleibt ECMO-abhängig und wird aus dem Bett mobilisiert.
Unter o.g. Therapie (10x Plasmapheresen) zeigt sich eine langsame, aber anhaltende klinische und laborchemische Besserung (anti-GBM $<20 \mathrm{U} / \mathrm{ml}$ ). Die ECMO-Therapie kann nach 48 Tagen beendet werden. In den ambulanten Verlaufskontrollen (zuletzt 08/2018) zeigt sich auch nach stufenweiser Reduktion der immunsuppressiven Therapie über 6 Monate und deren Beendigung kein Anhalt für ein Rezidiv der Grunderkrankung.

In ähnlichen Fallberichten wird die Freilegung der Basalmembran durch ein inhalatives Trauma (u.a. Tabak, Kokain, Speed) als pathophysiologischer Auslöser für das sekundäre Goodpasture-Syndrom diskutiert [1-5].

Auch bei zuvor negativem anti-GBM sollte ein schweres akutes Lungenversagen nach Hämoptysen, inhalativer Noxenkonsum und Panbronchiolitis im HRCT, zur erneuten Bestimmung des anti-GBM-Wertes leiten, und die seltene Erkrankung eines sekundären, ausschließlich pulmonalen Goodpasture-Syndroms in Erwägung gezogen werden.

[1] Cui Z, Zhao M-h, Singh AK et al. Antiglomerular basement membrane disease with normal renal function. Kidney Int 2007; 72: 1403-1408. doi:10.1038/sj.ki.5002525

[2] Chan AL, Louie S, Leslie KO et al. Cutting edge issues in Goodpasture's disease. Clin Rev Allergy Immunol 2011; 41: 151-162. doi:10.1007/ S12016-010-8222-2

[3] Balke L, Both $M$, Arlt A et al. Severe adult respiratory distress syndrome from Goodpasture syndrome. Survival using extracorporeal membrane oxygenation. Am J Respir Crit Care Med 2015; 191: 228-229. doi:10.1164/rccm.201409-1625IM

[4] Legras A, Mordant P, Brechot $\mathrm{N}$ et al. Prolonged extracorporeal membrane oxygenation and lung transplantation for isolated pulmonary anti-GBM (Goodpasture) disease. Intensive Care Med 2015; 41: 1866-1868. doi:10.1007/s00134-015-3983-y

[5] Rosa N de, Glanville A. ECMO-dependent respiratory failure after snorting speed associated with anti-GBM antibodies. Respirol Case Rep 2015; 3: 138-140. doi:10.1002/rcr2.128

\section{PS076}

\section{Attributable Mortality of Candidemia after Introduction} of Echinocandins

Florian Cornely; Oliver Cornely; Philipp Koehler; Felix Koehler; Sibylle Mellinghoff

Uniklinik Köln, Innere Medizin, Köln, Germany

Background: Candidemia is among the most frequent nosocomial blood stream infections and associated with considerable morbidity and mortality. Landmark case-control studies estimated an attributable mortality of $38 \%$ and $49 \%$. After introduction of echinocandins, attributable mortality may have decreased.

Methods: In a case-control design, 100 consecutive, hospitalized patients with candidemia were enrolled at the University Hospital of Cologne. These cases had at least one blood culture positive for Candida sp. $>48$ hours post admission. We enrolled patients from Jan 2017 backwards until Feb 2014. Controls were patients without candidemia matched for age, sex, calendar year, duration of hospitalization, main admission diagnosis, and Patient Clinical Complexity Level. Risk factors for candidemia captured were malignancy, diabetes, infection other than candidemia, liver cirrhosis, hemodialysis, congestive heart 
failure, coronary artery disease, chronic lung disease, intensive care, mechanical ventilation, and presence of central lines. For each control patient we evaluated the day during hospitalization on which the matched case had its onset of candidemia and then compared both groups concerning the post diagnosis length of stay. We estimated attributable mortality until day 30 post candidemia diagnosis. We performed $\mathrm{X}^{2}$-test for categorical and Students $\mathrm{t}$-test for continuous variables, and defined a two-tailed $\mathrm{p}$-value $<0.05$ statistically significant.

Results: Cases and controls were $68 \%$ males. Median age was 62 and 63 years, and 25th and 75th percentile 55 and 74 years in both groups. Candidemia occurred a median 18 days post admission.

For cases and controls, median length of stay post diagnosis was 17 and 15.5 days $(p=0.13)$, for those who died 12 and 19 days $(p=0.21)$, and for survivors 24 and 13 days $(p=0.006)$. Day 30 mortality rates were $38 \%$ and $11 \%$ for cases and controls $(p=0.03)$, thus attributable mortality was $27 \%$.

Conclusion: Attributable mortality of nosocomial candidemia is still substantial, but was lower in our study as compared to literature from before introduction of echinocandins.

\section{PS077 \\ Extrazellulärmatrix reduziert die Visfatin-vermittelten osteodestruktiven Effekte bei der adipogenen MSC-Differenzierung}

Lali Tsiklauri'; Janina Werner ${ }^{2}$; Klaus Frommer ${ }^{3}$; Stefan Rehart ${ }^{4}$; Sabine Wenisch ${ }^{2}$; Ulf Müller-Ladner ${ }^{3}$; Elena Neumann ${ }^{3}$

'Campus Kerckhoff, Bad Nauheim, Justus-Liebig-Universität Gießen, Rheumatologie und Klinische Immunologie, Bad Nauheim, Germany; ${ }^{2}$ Institut für Veterinär-Anatomie, -Histologie und -Embryologie, JLU Gießen, Klinik für Kleintiere, Giessen, Germany; ${ }^{3}$ Campus Kerckhoff, Bad Nauheim, JLU Gießen, Abteilung Rheumatologie und Klinische Immunologie, Dan Nauheim, Germany; ${ }^{4}$ Agaplesion Markus-Krankenhaus, Frankfurt, Klinik für Orthopädie und Unfallchirurgie, Frankfurt, Germany

Hintergrund: Bei der Osteoporose ist der altersbedingte Knochenverlust mit erhöhter Fettinfiltration im Knochenmark assoziiert. Somit wird das osteoporotisch veränderte Hartgewebe direkt von den Adipozyten des Knochenmarks begrenzt. Angesichts der hohen sekretorischen Aktivitäten der Adipozyten liegt die Hypothese nahe, dass adipozytäre Sekretionsfaktoren wie Adipokine (z.B. Visfatin, Leptin und Resistin) die Differenzierung der im Knochenmark befindenden MSC beeinflussen.

Ziel: Analyse des Expressionsprofils der Adipokine im Knochengewebe sowie deren Einfluss auf die adipogene MSC-Differenzierung in Standardkultur sowie auf Spongiosa.

Methoden: Aus der Spongiosa humaner Hüftköpfe von Patienten mit Schenkelhalsfraktur (FF) oder mit Arthrose (OA) wurde RNA sowie MSC isoliert und Genexpressionsanalysen mittels Real-Time PCR durchgeführt. Für Spongiosa-Transfer-Experimente wurde die Spongiosa zellfrei gereinigt und sterilisiert. Die MSC-Differenzierung erfolgte über 21 Tagen mit bzw. ohne Zugabe von Adipokinen, sowie Visfatin- (Apo866) und p38-MAPK (SB203580) Inhibitoren. Die Messung der Proteinexpression erfolgte mittels ELISA.

Ergebnisse: Visfatin und Leptin waren in der FF-Gruppe erhöht. Visfatin induzierte die Expression von MMP13 auf mRNA und Proteinebene während der Adipogenese in Standardkultur. Im Vergleich dazu zeigte sich die Visfatin-induzierte MMP13 Expression während der Adipogenese auf Spongiosa deutlich erniedrigt (z.B. x-folds, Tag 21:
Standardkultur 81 \pm 89 , Spongiosa $13 \pm 21, \mathrm{n}=7$ ). Die Visfatin-induzierte Expression von Entzündungsmediatoren war in Standardkultur als auch auf Spongiosa in adipogen differenzierten Zellen nachweisbar, jedoch geringer bei Zellen, die auf Spongiosa differenzierten (z.B. x-folds, IL6, 14d: Standardkultur 152 \pm 110 , Spongiosa $41 \pm 31, n=7)$. In Standardkultur führte die Zugabe von Apo866 zur reduzierten Freisetzung von Entzündungsmediatoren, während die Visfatin-induzierte MMP13 Expression nicht beeinflusst wurde. Die Hemmung von p38 MAPK beeinflusste nicht die Zytokinexpression.

Schlussfolgerungen: Visfatin und Leptin werden vermehrt in FF- als in OA-Knochen exprimiert. Visfatin induziert die Freisetzung von Matrix-abbauenden Proteinen während der Adipogenese und könnte somit zu einem gestörten Knochenremodelling beitragen. Die Extrazellulärmatrix wirkt abschwächend auf die Visfatin-induzierten osteodestruktiven Effekte. Möglicherweise sind die verschiedenen Signalwege für die Visfatin-vermittelten Effekte verantwortlich.

\section{PS078}

\section{Severe serotonine Syndrom under Venlafaxin therapy}

Hauke Tews

Gastroenterologie UKSH Lübeck, Neustadt, Germany

A 27-year-old patient with serotonin syndrome under Venlafaxine therapy

A 27-year-old female, who was accompanied by her boyfriend, introduced herself to the emergency unit on the 10th of May 2018. Her boyfriend reported that she had sent him a farewell message via her smartphone. He also reported that he had found an empty tablet blister and an empty bottle of Ouzo.

The hospital admittance was based on a mixed intoxication. Clinical signs of a serotonin syndrome, like the flipping eye syndrome, tachycardia, akathisia and tremor were noticeable, suited to an intoxication with venlafaxine. A blood alcohol concentration of 2,32 per thousand was detected as well. During the first hours of therapy at the intensive care unit the patient was hemodynamically stable.

The next day the symptoms of the serotonin syndrome aggravated, leading to uncontrolled motoric movements with extreme hyperreflexia. A progressive cardiogenic shock with multiple organ failure (kidney, liver, heart), lactic acidosis and rhabdomyolysis developed. In spite of consumption of the conservative methods, a stabilization of the circulation could not be reached.

As ultima ratio, an assistance for the circulation via ECLS (A. and V. femoralis dextra) was initiated.

A few hours later, a massive bleeding out of the puncture of the arterial ECLS needle existed. In spite of the surgical intervention, it came to a hemorrhagic shock.

As a result, the needles for the ECLS were changed from the right inguinal side to the left inguinal side, and the vessels on the right side were sewed. In the course of time an acute kidney failure with anuria and metabolic acidosis developed, so that a hemodialysis was initiated. In the following hours, the dosis for the Catecholamin therapy increased rapidly. Using a volume replacement and an antibiotic therapy with Meropenem and Vancomycin by unknown focus, a haemodynamic stabilization could be reached.

After a few days we explanted the ECLS and documented a good systolic heart function. During the course of staying at the intensive care 
unit, the patient developed a diuresis und by decreasing kidney retention parameters we finished hemodialysis.

In summary, we treated a 27-year-old patient with multiple symptoms of a serotonin syndrome with multiple organ failures by a mixed intoxication with Venlafaxin (at least 13,5 grams), Ibuprofen (6 grams) and alcohol in suicidal intention. The artificial respiration outlasted for 13 days. By the clinic of a cardiogenic shock, based on a toxic kardiomyopathie, a treatment via ECLS was realized from the 11th of May 2018 to the 17 th of May 2018.

With regard to the ECLS therapy, an artificial respiration and an intermitting hemodialysis, several methods were indispensable for stabilising the patient.

Fortunately, the patient was discharged after 20 days of habitation at the intensive care unit. After approximately 20 days at the intensive care unit, we saw the clinical picture of a "restutio ad integrum".

\section{PS079 \\ GLP-1 levels predict cardiovascular risk in patients with acute myocardial infarction}

Florian Kahles'; Marcia Vivianne Rückbeil'; Robert Werner Mertens'; Ann Christin Foldenauer ${ }^{2}$; Maria Chiara Arrivas'; Julia Moellmann'; Corinna Lebherz'; Moritz Biener ${ }^{3}$; Evangelos Giannitsis ${ }^{3}$; Hugo Albert Katus ${ }^{3}$; Nikolaus Marx'; Michael Lehrke'

'Uniklinik RWTH Aachen, Medizinische Klinik I (Kardiologie), Aachen, Germany; ${ }^{4}$ Uniklinik RWTH Aachen, Institut für medizinische Statistik, Aachen, Germany; ${ }^{2}$ Fraunhofer Institute for Molecular Biology and Applied Ecology IME Frankfurt, Fraunhofer Institute for Molecular Biology and Applied Ecology IME, Frankfurt am Main, Germany; ${ }^{3}$ Uniklinikum Heidelberg, Klinik für Kardiologie, Angiologie und Pneumologie, Heidelberg, Germany

Background: Glucagon-like peptide 1 (GLP-1) is a gut incretin hormone, which induces post-prandial glucose-dependent insulin secretion. GLP-1 receptor agonists are used for the treatment of type 2 diabetes and have been found to improve cardiovascular outcomes in patients with diabetes at high cardiovascular risk. We recently found GLP-1 levels to be increased in patients with acute myocardial infarction. The aim of this study was to assess the predictive capacity of GLP-1 for cardiovascular outcome in patients with myocardial infarction.

Methods: Total GLP-1 levels, NT-proBNP concentrations and the Global Registry of Acute Coronary Events (GRACE) score were assessed at time of admission in 918 patients with myocardial infarction presenting with acute chest pain. Among these 597 patients presented with NSTEMI and 321 with STEMI. The primary composite outcome of the study was the first occurrence of all-cause death, nonfatal myocardial infarction, or nonfatal stroke with a median follow-up of 311 days.

Results: Kaplan-Meier survival plots (separated by tertiles with cut-off values 35.44 and 53.45 ) and univariate cox regression analyses found GLP-1 values to be associated with adverse outcome (combined endpoint and all-cause mortality) (logarithmized GLP-1 values HR: 5.459; $p<.0001$ ). Further adjustment for age, sex, previous cardiovascualar disease, diabetes, hypertension, hypercholesterinemia, kreatinin, CRP, troponin T and NT-proBNP levels did not affect the association of GLP-1 with adverse outcomes $(p=0.0341)$. Receiver operating characteristic curve (ROC) analyses illustrated that GLP-1 is a strong indicator for early events (area under the curve of the combined endpoint at 15 days: 0.78 ; 30 days: $0.73 ; 183$ days: 0.62 ), which proved to be superior to Troponin T. Adjustment of the GRACE risk estimate by GLP-1 increased the area under the receiver-operating characteristic curve (AUC) after 1 month from 0.86 to 0.89 in NSTEMI patients. Addition of GLP-1 to a model containing GRACE and NT-proBNP led to a further improvement in model performance (increase in AUC from o.88 for GRACE + NT-proBNP to 0.90 for GRACE + NT-proBNP + GLP-1).

Conclusion: GLP-1 is a new biomarker of cardiovascular risk and adverse outcomes in patients with acute myocardial infarction and improves the predictive value of the GRACE score in patients with NSTEMI.

\section{PS080 \\ Korrelation der Leberperfusion mit Klinik und Outcome von Patienten mit schweren Lebererkrankungen - eine monozentrische prospektive Kohortenstudie auf der Intensivstation}

Johannes Vogg '; Constantin Maier-Stocker'; Lukas Moleda ${ }^{2}$; Karsten Gülow'; Martina Müller-Schilling'; Stephan Schmid'

'Universitätsklinikum Regensburg, Klinik für Innere Medizin I, Regensburg, Germany; ${ }^{2}$ Uniklinikum Regensburg, Klinik und Poliklinik für Innere Medizin I, Regensburg, Germany

Hintergrund: Chronische Lebererkrankungen sind eine der Hauptursachen für Morbidität und Mortalität weltweit. Pathophysiologisch führen Entzündungsprozesse zur hepatozellulären Schädigung und Fibrosierung des Lebergewebes. Die intrahepatischen Widerstände erhöhen sich und es resultiert eine Veränderung der Hämodynamik hepatischer Blutgefäße. Die Sonographie spielt eine besonders wichtige Rolle für die Evaluation von Lebererkrankungen. Jedoch ist die präzise Rolle und der prognostische Wert einer routinemäßig durchgeführten Doppler-Sonographie der Leberperfusion bei Patienten mit schweren Lebererkrankungen noch nicht ausreichend geklärt.

Zielsetzung: Ziel der Studie ist es, eine mögliche Korrelation der Leberperfusion mit Klinik und Outcome von Patienten mit schweren Lebererkrankungen im Rahmen einer intensivmedizinischen Behandlung zu analysieren.

Patienten und Methoden: 50 Patienten, die mit schweren Lebererkrankungen im Jahr 2018 auf der internistischen Intensivstation der Klinik und Poliklinik für Innere Medizin I des Universitätsklinikums Regensburg hospitalisiert waren, wurden routinemäßig zweimal wöchentlich während ihrer intensivmedizinischen Behandlung sonographisch untersucht. Zur Quantifizierung der Leberperfusion wurde der hepatic artery resistance index (HARI) und die maximale portal vein velocity (PVv) mittels Doppler-Ultraschall ermittelt. Zum Zeitpunkt der Untersuchung wurde für jeden Patienten der MELD-Score (Model for End-stage Liver Disease) errechnet. Außerdem wurden weitere klinische Daten wie die aktuelle Katecholamindosis, der mittlere arterielle Blutdruck sowie Beatmungs- und Laborparameter erhoben. Weiterhin wurden auch die Dauer der intensivmedizinischen Behandlung und das Outcome der Pateinten mit den sonographischen Parametern korreliert.

Ergebnisse: Analysen von MELD-Score, HARI und max. PVv zeigen eine lineare, positive Korrelation zwischen HARI und MELD-Score. Zwischen maximaler PVv und MELD-Score zeigt sich eine lineare negative Korrelation. Regressionsanalysen quantifizieren diese Zusammenhänge mit einem $\mathrm{R}^{\wedge}{ }_{2}$-Wert von 0,220 (HARI - MELD-Score) und 0,078 (PVv - MELD-Score). Weiterhin zeigt sich, dass HARI bei Patienten, die im Laufe der stationären Behandlung verstarben, durchschnittlich um 1,6\% mit jeder Untersuchung stieg, dagegen bei nicht verstorbenen Patienten um $0,3 \%$ sank. Im Mittel stieg die maximale PVv mit jeder Untersuchung bei verstorbenen Patienten um 1,8\% und um $16,5 \%$ bei nicht verstorbenen Studienteilnehmern. 
Zusammenfassung: Die statistisch signifikante Korrelation von HARI und maximaler PVv mit dem MELD-Score zeigt, dass die Entwicklung der Leberperfusion im Verlauf einen prognostischen Faktor für schwere Lebererkrankungen darstellt. Die routinemäßige Erfassung von HARI und max. PVv bei Patienten mit schweren Lebererkrankungen auf der Intensivstation ist damit weiter zu evaluieren.

\section{Pneumologie}

\section{PS081}

Einfluss der Tumorlast auf den Überlebensvorteil einer immunonkologischen Behandlung bei Nicht-kleinzelligem Lungenkrebs (NSCLC): Fall-Kontroll-Analyse des Überlebens (OS) in der klinischen Routine

Marcel Kopp'; Birgit Schwenk'; Sabine Fallscheer'; Sebastian Kramberg'; Matthias Leschke'; Robert Eckert ${ }^{2}$; Martin Faehling ${ }^{1}$

'Klinikum Esslingen, Klinik für Kardiologie und Pneumologie, Esslingen, Germany; ${ }^{2}$ Onkologische Schwerpunktpraxis, Onkologie, Esslingen, Germany

Hintergrund: Immun-onkologische (IO) Therapien mit PD-1- und PD-L1-Antikörpern sind seit 2015 zur Behandlung des fortgeschrittenen NSCLC zugelassen worden, nachdem in randomisierten Studien Überlebensvorteile mit Hazard ratios (HR) oft zwischen o,5 und o,6 nachgewiesen worden waren. Der Einfluss der IO-Therapien auf das Überleben in der klinischen Routine und die Bedeutung der Tumorlast sind nicht untersucht worden.

Methoden und Patienten: Untersucht wurden 1334 Patienten, die in unserem DKG-zertifizierten Lungenkrebszentrum von 2006 bis September 2018 mit NSCLC diagnostiziert wurden. Das OS der Patienten, welche mindestens einen Zyklus einer immunonkologischen Therapie mit PD-1-Antikörpern (Nivolumab $[n=76]$ oder Pembrolizumab $[n=51]$ ) oder PD-L1-Antikörpern (Atezolizumab $[n=4]$ oder Durvalumab [ $n=12]$ ) erhalten haben ("IO-Patienten"), wurde in einer Fall-Kontroll-Analyse mit historischen Kontrollen, die vor der Verfügbarkeit von IO-Therapien am Zentrum behandelt worden waren, verglichen. Die Fall-Kontroll-Paare stimmten in Geschlecht, Histologie, Stadium, Allgemeinzustand (ECOG), Raucherstatus und initialem Behandlungsmodus (palliativ vs. kurativ) überein. Die Zuordnung erfolgte verblindet in Bezug auf das Überleben. Bei mehreren passenden Kontrollen wurde die mit dem geringsten Altersunterschied zugeordnet. Für 109 der 143 IO-Patienten war eine passende Kontrolle vorhanden. Ein positives Ethikvotum liegt vor (Landesärztekammer Baden-Württemberg F-2017-004).

Ergebnisse: IO-Patienten wiesen ein signifikant längeres medianes Gesamtüberleben im Vgl. zu historischen Kontrollen auf (21,8 vs. 10,7 Monate, $\mathrm{HR} 0,55, \mathrm{Cl} 0.40$ 0.75, $\mathrm{p}=0.0002$ ). Der Überlebensvorteil war größer bei Patienten mit niedrigerem Tumorstadium (HR 0,364 [Stadium III], 0,407 [IVA], 0,605 [IVB]) oder kleinerer Tumorgröße (HR 0,364 [RECIST < 75], 0,443 [RECIST 75-121], 0,761 [RECIST > 121]).

Schlussfolgerung: IO-Patienten hatten in der klinischen Routine einen deutlichen Überlebensvorteil mit Hazard ratios, die denen in den publizierten Phase III-Studien vergleichbar waren. Der Überlebensvorteil war bei Patienten mit niedrigerer Tumorlast ausgeprägter.

\section{PS082}

Feasibility and accuracy of SPECT myocardial perfusion imaging in end-stage lung disease

Sanziana Schiopu'; Mathias Zacherl5; ; Andrei Todica ${ }^{5}$; Katrin Milger'; Gabriela Leuschner'; Dieter Munker'; Steffen Massberg ${ }^{3}$; Juergen Behr'; Claus Neurohr ${ }^{2}$; Bruno Huber ${ }^{3}$; Nikolaus Kneidinger ${ }^{4}$

'Ludwig-Maximilians-Universität München, Medizinische Klinik V, Munich, Germany; ${ }^{2}$ Robert-Bosch-Krankenhaus, Klinik Schillerhöhe, Abteilung für Pneumologie und Beatmungsmedizin, Gerlingen, Germany; ${ }^{3}$ Ludwig-Maximilians-Universität München, Medical Department I, Campus Grosshadern and Campus Innenstadt, Munich, Germany; ${ }^{4}$ Ludwig-Maximilians-Universität München, Medical Department V, Munich, Germany;5udwig-Maximilians-Universität München, Department of Nuclear Medicine, Munich, Germany

Background: Coronary artery disease (CAD) is associated with increased mortality in patients with chronic lung diseases. However, non-invasive diagnostic of CAD is limited, especially in patients with more advanced disease. Therefore, we aimed to assess the feasibility and accuracy of SPECT-myocardial perfusion imaging (MPI) stress testing with regadenoson in patients with end-stage lung disease (ELD) undergoing assessment of stable CAD.

Methods: In total, 102 consecutive patients with ELD were assessed retrospectively from 2012 to 2018 . All patients underwent both SPECT$\mathrm{MPI}$ and coronary angiography as part of a lung transplant evaluation.

Results: The mean age was $58 \pm 6$ years. Mean forced vital capacity and forced expiratory volume in 1 second were $50 \pm 16$ and $36 \pm 20 \%$ predicted, respectively. $86 \%$ of patients required long-term oxygen therapy. Regadenoson-related symptoms requiring medical intervention were observed in 2 patients $(2 \%)$ and 14 patients (14\%) reported symptoms. In total, 8 patients (8\%) had abnormal SPECT-MPI and revascularisation was performed in 6 patients $(6 \%)$. Specificity and negative predictive value of SPECT-MPI were $95 \%$ and $98 \%$, respectively and were not influenced by confounding factors.

Conclusions: Our findings suggest that SPECT-MPI stress testing with regadenoson is well tolerated and has a high value to rule out CAD in patients with ELD.

\section{PS083 \\ Effect Of Endotoxin On The Response To Chemotherapy And Radiotherapy In NSCLC Cell Lines}

Julia D. Lampey'; Ulrich Grandel²; Mira Y. Goekyildirim; Gabriele Dahlem; Friedrich Grimminger ${ }^{2}$; Ulf Sibelius'; Katja Hattar ${ }^{1}$

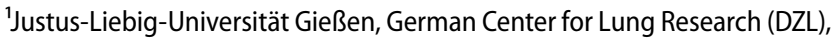
Cardio-Pulmonary Institute (CPI), Medizinische Klinik IV/V, Gießen, Germany; ${ }^{2}$ Justus-Liebig-Universität Gießen, German Center for Lung Research (DZL), Cardio-Pulmonary Institute (CPI); Asklepios Klinik Lich, Medizinische Klinik IV/V, Gießen, Germany

Background: Lung cancer is the leading cause of cancer death in Germany. In this context therapy resistance promoted by inflammation could play an important role. The relationship between inflammation and tumor progression has been known for a long time. Bacterial lipopolysaccharides (LPS) are an important pathogenicity factor present in Gram-negative bacteria. Their proliferative effects on NSCLC cell lines have been already demonstrated. 
Methods: We investigated the effect of LPS on the response to chemotherapy and radiotherapy in vitro using the human adenocarcinoma cell lines $\mathrm{A}_{549}$ and $\mathrm{H}_{1975}$. The cells were incubated with LPS (0.1, $1,10 \mu \mathrm{M}$ ) and treated with cisplatin (various dosages) and/or ionizing radiation. The clonogenic survival was determined by colony formation assay. To analyze the relative levels of phosphorylation of different kinases in relation to exposure to LPS and ionizing radiation a human phospho-kinase array was performed. Furthermore cellular IL-8-synthesis after LPS-incubation, irradiation and inhibitor-treatment was quantified by ELISA.

Results: Neihter A549 cells nor $\mathrm{H} 1975$ cells showed a reduced response to cisplatin after incubation with LPS. The A549 cells exposed to $10 \mu \mathrm{M}$ LPS showed a significant decreased response to radiotherapy. Further investigations showed that the relative phosphorylation levels of ERK $1 / 2$ were increased significantly after exposure to LPS. Moreover, inhibition of $E R_{1} / 2$ resulted in a restoration of radiosensitivity of A549 cells even in the presence of LPS. After exposure to LPS the cellular synthesis of IL-8 was increased significantly, especially if cells were additionally treated with ionizing radiation, whereas the application of the ERK-inhibitor led to a reduction in levels of IL-8.

Conclusion: LPS reduce radiosensitivity of A549. The effect of LPS on therapy resistance is mediated by ERK $1 / 2$ and possibly by IL-8. These findings could be of clinical importance, as NSCLC patients who suffer from infections could develop radiotherapy resistance and tumor progression. Interference with the ERK-pathway may be a promising option to restore radiosensitivity in NSCLC patients.

\section{PS084 \\ Akute Dyspnoe mit Milchglastrübung im Lungen-CT bei einer 34-jährigen Patientin mit Rheumatoider Arthritis}

Hanne Wallacher; Jens Bester; Matthias Hillebrand; Christoph Rieder-Nelissen; Peter Härle

Katholisches Klinikum Mainz, Klinik für Rheumatologie, Klinische Immunologie und Physikalische Therapie, Nierstein, Germany

Fallpräsentation: Eine 34-jährige Patientin stellte sich im November 2018 mit vor 3 Wochen akut begonnener Belastungsdyspnoe und trockenem Husten nach Einweisung durch den Pneumologen über unsere ZNA vor. An Vorerkrankungen waren eine ACPA-positive Rheumatoide Arthritis (ED 2017), Migräne und Katzenhaarallergie bekannt. Wegen fehlender Remission unter inital konventioneller DMARDs (MTX, Sulfasalazin und HCQ) und Kinderwunsch bestand seit Mai 2018 eine Anti-TNF-Therapie mit Certolizumab. Hierauf war rasch eine klinische Remission eingetreten. Eine weitere Medikation bestand nicht. Es ergab sich kein Hinweis auf eine Allergen oder anderweitige inhalative Noxen-Exposition. Diagnostik: In der Untersuchung sahen wir eine Patientin in reduziertem AZ und Sprechdyspnoe. Pulmonal ließ sich bds. ein vesikuläres Atemgeräusch auskultieren. Die BGA zeigte eine resp. Partialinsuffizienz ( $\mathrm{pO}_{2} 58.3 \mathrm{mmHg}$ ). Laborchemisch fielen leicht erhöhte CRP $(8 \mathrm{mg} / \mathrm{l})$ und LDH-Werte $(342 \mathrm{U} / \mathrm{ml})$ auf bei normwertiger BSG und Leukozyten. Das ambulant durchgeführte CT Thorax zeigte eine homogene milchglasartige Trübung beider Lungen, lungenfunktionell bestand eine mittelgradige Restriktion und schwergradige Diffusionsstörung. Es wurde eine Bronchoskopie mit BAL und transbronchialer Biopsie durchgeführt. Die mikrobiologische Lavage blieb steril, zudem kein Anhalt auf eine CMV- oder PjP-Infektion. Die BAL zeigte eine ausgeprägte lymphozytäre Alveolitis. Die TBB zeigte peribronchial ein geringes subepitheliales lymphozytäres Infiltrat. Therapie und Verlauf: Unter dem Verdacht einer Certolizumab-induzierten Alveolitis wurde umgehend eine Glukokortikoidtherapie (6o mg Prednisolon) initiiert und das Certolizumab pausiert. Nach 2- 3 Tagen zeigte sich sowohl eine klinische Besserung als auch
Verbesserung der Lungenfunktion und BGA. Die Patientin konnte 7 Tage nach Entlassung mit einer Tagesdosis von $40 \mathrm{mg}$ Prednisolon nahezu beschwerdefrei entlassen werden. Zusammenfassung und Diskussion: Die Entwicklung einer akuten interstitiellen Lungenerkrankung unter anti-TNFa-Therapie ist eine seltene, aber bekannte Nebenwirkung. Unter Therapie mit Certolizumab sind seit der Erstzulassung (2008) allerdings erst 4 Fälle beschrieben. Hierunter findet sich ein letal verlaufender Fall, die weiteren mit persistierender Einschränkung der Lungenfunktion. Im Unterschied zu unserem Fall waren die Patienten deutlich älter (>60 J.) und längere Zeit erkrankt. 3 der 4 Patienten standen zuvor oder zeitgleich unter einer Therapie mit MTX und hatten eine vorbeschriebene Lungenerkrankung (COPD, MTX-Pneumonitis). Insbesondere bei Patienten mit Autoimmunerkrankungen und Immunsuppression ist die initiale Differenzierung zwischen Infektionskomplikation, pulmonaler Beteiligung der Grunderkrankung oder Nebenwirkung schwierig aber essentiell. Neben einer raschen Diagnosestellung ist ein zeitnaher Beginn mit hochdosierter Glukokortikoidtherapie für die Vermeidung möglicher Langzeitschäden entscheidend.

\section{PS085}

\section{LRP1 promotes synthetic phenotype of pulmonary artery smooth muscle cells in pulmonary hypertension}

Marius Michael Zucker'; Lukasz Wujak'; Anna Gungl'; Miroslava Didiasova'; Djuro Kosanovic ${ }^{6}$; Ralph Schermuly ${ }^{6}$; Aleksandar Petrovic ${ }^{6}$; Walter Klepetko $^{3}$; Grazyna Kwapiszewska ${ }^{4}$; Liliana Schäfer ${ }^{5}$; Malgorzata Wygrecka'

'Justus-Liebig-Universität Gießen, Institut der Biochemie, Gießen, Germany; ${ }^{2}$ Universtität Graz, Ludwig Boltzmann Institut, Lungengefäßforschung, Graz, Austria; ${ }^{3}$ Medizinische Universität Wien, Institut der Thoraxchirurgie, Wien, Austria; ${ }^{4}$ Medizinische Universität Graz, Ludwig Boltzmann Institut, Lungengefäßforschung, Graz, Austria; ${ }^{5}$ Goethe-Universität Frankfurt am Main, Institut der Pharmakologie, Frankfurt, Germany; ${ }^{6}$ Justus-Liebig-Universität Gießen, Innere Medizin, Gießen, Germany

Pulmonary hypertension $(\mathrm{PH})$ is characterized by the thickening of the distal pulmonary arteries caused by medial hypertrophy, intimal proliferation and fibrosis. Low density lipoprotein receptor-related protein1 (LRP1) has the capacity to maintain vascular homeostasis by mediating the endocytosis of numerous ligands and by initiating and regulating signaling pathways.

Here, we demonstrate the increased LRP1 protein levels in the lungs of idiopathic pulmonary arterial hypertension (IPAH) patients, hypoxia-exposed mice, and monocrotaline (MCT)-treated rats. Platelet-derived growth factor (PDGF)-BB upregulated LRP1 expression in pulmonary artery smooth muscle cells (PASMC). This effect was reversed by PDGF-BB neutralizing antibody or the PDGF-BB receptor antagonist Imatinib. Depletion of LRP1 decreased proliferation of donor and IPAH PASMC in a $\beta_{1}$-integrin-dependent manner. Furthermore, LRP1 silencing attenuated the protein expression of fibronectin and intercellular adhesion molecule 1 and increased the levels of alpha-smooth muscle actin (a-SMA) and myocardin in donor, but not in IPAH, PASMC. In addition, smooth muscle cell (SMC)-specific LRP1 knockout augmented a-SMA expression in pulmonary vessels and reduced SMC proliferation in $3 \mathrm{D}$ ex vivo murine lung tissue cultures.

In conclusion, our results indicate that LRP1 promotes dedifferentiation of PASMC from a contractile to a synthetic phenotype thus suggesting its contribution to vascular remodeling in $\mathrm{PH}$. 
PS086

\section{Effect of COPD severity and comorbidities on the result of the PHQ-9 tool for the diagnosis of depression: Results from the COSYCONET cohort study}

Marietta von Siemens'; Rudolf A. Jörres'; Jürgen Behr ${ }^{8}$; Peter Alter ${ }^{2}$; Johanna Lutter ${ }^{3}$; Sandra Söhler ${ }^{4}$; Tobias Welte ${ }^{5}$; Henrik Watz ${ }^{6}$; Franziska Trudzinski'; Winfried Rief ${ }^{9}$; Britta Herbig ${ }^{1}$; Kathrin Kahnert ${ }^{8}$

'Klinikum der Universität München, Institute and Outpatient Clinic for Occupational, Social and Environmental Medicine, München, Germany; ${ }^{2}$ Philipps-University Marburg, Department of Medicine, Pulmonary and Critical Care Medicine, Marburg, Lithuania; ${ }^{3}$ Helmholtz Zentrum München $\mathrm{GmbH}$, Institute of Health Economics and Health Care Management, München, Germany; ${ }^{4}$ University of Marburg, ASCONET Study Coordination Office, Marburg, Ghana; ${ }^{5}$ Hannover Medical School, Department of Pneumology, Hannover, Gibraltar; ${ }^{6}$ Pulmonary Research Institute at LungenClinic Grosshansdorf, Airway Research Center North, Grosshansdorf, Germany; ${ }^{7}$ Saarland University Hospital,Department of Internal Medicine V - Pulmonology, Allergology, Respiratory Intensive Care Medicine, Homburg, Germany; ${ }^{8}$ Klinikum der Universität München, Medizinsiche Klinik V, München, Germany; ${ }^{9}$ University of Marburg, Klinische Psychologie und Psychotherapie, Marburg, Germany

The diagnosis of depression, a frequent comorbidity of COPD, is often supported by questionnaires, such as the Patient Health Questionnaire 9 (PHQ-9). It is unknown to which extent its single questions are affected by the characteristics of COPD patients.

We addressed this question in 2255 GOLD grade 1-4 patients from the COSYCONET COPD cohort. The dependence on COPD severity was assessed using symptoms, exacerbation risk (GOLD A-D; mMRC), and frequent comorbidities as predictors of $\mathrm{PHQ}-9$ results, while including age, gender, BMI and smoking habits as covariates.

Symptoms and exacerbation risk were associated in an additive manner, with mean elevations in the PHQ-9 sum score by 2.75 and 1.44 points, respectively. Asthma, sleep apnoea, gastrointestinal disorders, osteoporosis and arthritis were linked to increases by 0.8 to 1.3 points. Overall, the COPD characteristics contributed to the mean score by increases from 4.5 or 5.2 to 6.3 points, respectively, when either taking GOLD A as reference or the absence of comorbidities, independent of the diagnosis of mental disorder or intake of antidepressants. The presence of COPD led to an increase in the proportion of scores indicating depression from 12 to $22 \%$. Single item analysis revealed homogenous effects regarding GOLD groups, but heterogeneous effects regarding other COPD characteristics.

These findings indicate specific effects of COPD severity, especially symptoms and exacerbation risk, on the $\mathrm{PHQ}-9$ depression score, explain the high prevalence of depression in COPD. Alternatively, our findings raise the question of a bias from COPD severity on the PHQ-9, but also suggest COPD treatment effects on depression scores.

\section{PS087}

High eosinophil blood counts are associated with a shorter length of hospital stay in exacerbated COPD patients a retrospective analysis

Julia Tüffers'; Sina Mager ${ }^{3}$; Anna Eder ${ }^{3}$; Peter Alter ${ }^{2}$; Claus Franz Vogelmeier'; Timm Greulich ${ }^{4}$

${ }^{1}$ Philipps-Universität Marburg, Klinik für Innere Medizin, Pneumologie, Marburg, Germany; ${ }^{2}$ Philipps Universität Marburg/ Klinik für Innere Medizin, Pneumologie, Deutsches Zentrum für Lungenforschung, Marburg, Germany; ${ }^{3}$ Philipps-Universität Marburg, Pneumologie, Marburg, Germany; ${ }^{4}$ Philipps Universität Marburg/ Klinik für Innere Medizin, Pneumologie, Marburg, Germany

Background: Chronic Obstructive Pulmonary Disease (COPD) is characterized by an irreversible airway obstruction. The course of the disease including morbidity and mortality is strongly associated with severe exacerbations.

The current international GOLD recommendations emphasize blood eosinophils as marker for responsiveness to inhaled corticosteroids (ICS). The recommended thresholds are $<100$ cells $/ \mu$ land $>300$ cells $/ \mu$ l.

Retrospective analyses from randomized clinical trials as well as prospective clinical trial data indicate a favorable response to systemic corticosteroids in exacerbated COPD patients with blood eosinophils $>2 \%$, however data outside clinical trials are scarce.

We thus aimed to examine the outcome of severe COPD exacerbations measured as length of hospital stay (LOS) according to the blood eosinophil count in a real life setting.

Patients and methods: In this preliminary analysis, we present retrospectively collected data from 374 patients who were admitted to the University Hospital Marburg between 01/2013 and 05/2015. All patients had been diagnosed with an acute exacerbation of COPD according to the ICD code (J44.0/J44.1). Patients were predominantly male $(67.4 \%)$, had a mean age of $74.2 \pm 11.8$ years and a mean FEV 1 of $1.21 \pm$ 0.6 I ( $46.9 \pm 19.8 \%$ predicted). This analysis was based on a subgroup of 262 patients in whom a full blood cell count was obtained at day of admission. These patients exhibited similar baseline characteristics (66.8\% male, $74.2 \pm 11.7$ years of age, FEV1 $1.22 \pm 0.65$ l, $47.1 \pm 19.7 \%$ predicted).

We compared the hospital length of stay as well as other markers of disease severity using various established thresholds for the eosinophil blood cell count (100 and 300 eosinophils/ $\mu$ land $2 \%$ ).

Results: Patients with low eosinophils ( $<2 \%,<100$ cells $/ \mu$ l) had a shorter median time in hospital (length of hospital stay - LOS) as compared to patients with high eosinophils. This was true for both relative (7 vs. 9 days; $p<0.05$ ) and absolute values ( 7 vs. 8 vs. 10 days; $p<0.05$ ). In contrast, the median CRP was higher in patients with low eosinophils as compared to the other groups ( <2\%: $33 \mathrm{mg} / \mathrm{dl}$ vs. $\geq 2 \%: 14.5$ $\mathrm{mg} / \mathrm{dl} ; \mathrm{p}<0.005)$. There were no differences in age, Procalcitonin, or case mix index (CMI) between the low eosinophil groups and their respective control groups.

Conclusions: The data support the hypothesis that patients with low eosinophil counts may be less responsive to systemic corticosteroids when compared to patients with high eosinophils. Thus, the data add to the growing body of evidence that blood eosinophils are useful as a biomarker for both inhaled and systemic steroid responsiveness. 


\section{PS088 \\ High-sensitivity troponin I predicts all-cause mortality in stable COPD in the COSYCONET cohort study}

Benjamin Waschki'; Peter Alter²; Tanja Zeller'; Christina Magnussen; Johannes Neumann'; Christian Herr ${ }^{3}$; Stefan Blankenberg'; Klaus Rabe ${ }^{4}$; Tobias Welte ${ }^{5}$; Rudolf A. Jörres ${ }^{6}$; Claus Franz Vogelmeier ${ }^{7}$; Robert Bals ${ }^{3}$; Henrik Watz $^{8}$

'University Heart Center Hamburg, Department of General and Interventional Cardiology, Hamburg, Germany; ${ }^{2}$ Philipps-University Marburg, Department of Medicine, Pulmonary and Critical Care Medicine,, Marburg, Germany; ${ }^{3}$ Saarland University Hospital, Department of Internal Medicine V - Pulmonology, Allergology, Critical Care Care Medicine, Homburg, Germany; ${ }^{4}$ LungenClinic Grosshansdorf, Department of Respiratory Medicine, Grosshansdorf, Germany; ${ }^{5}$ Medizinische Hochschule Hannover, Department of Respiratory Medicine, Hannover, Germany; ${ }^{6}$ Comprehensive Pneumology Center Munich, Institute and Outpatient Clinic for Occupational, Social and Environmental Medicine München, München, Germany; ${ }^{7}$ University Medical Center Giessen and Marburg, Department of Medicine, Pulmonary and Critical Care Medicine, Marburg, Germany; ${ }^{8}$ Pulmonary Research Institute at LungenClinic Grosshansdorf, Pulmonary Research Institute at LungenClinic Grosshansdorf, Grosshansdorf, Germany

Background: Cardiovascular disease is a frequent comorbidity and a major cause of death in COPD. High-sensitivity troponin I (hs-Tnl) is a powerful predictor for cardiovascular events and all-cause mortality in the general population. The aim of this study was to evaluate the predictive value of hs-Tnl for all-cause mortality in stable COPD.

Methods: In the multicenter COSYCONET cohort study, hs-Tnl concentrations together with a wide range of COPD assessments, cardiovascular risk factors and cardiovascular diseases were evaluated in 2085 patients with stable COPD (GOLD stage I-IV). Cox regression analyses were used to evaluate the predictive value of hs-Tnl for all-cause mortality over 3 years of follow-up.

Results: Hs-Tnl was detectable in 2020 (96.9\%) patients. The median hs-Tnl level was $3.8 \mathrm{ng} / \mathrm{L}$ (IQR, 2.5-6.6 ng/L). After adjustments for COPD assessments (e.g., airflow limitation, dyspnea grade, exercise capacity, and history of exacerbations), cardiovascular risk factors, and prevalent cardiovascular diseases, hs-Tnl was a significant predictor for all-cause mortality (adjusted hazard ratio for log hs-Tnl 1.28 [95\% $\mathrm{Cl}, 1.01-1.62]$ and for $\mathrm{hs}-\mathrm{Tnl}>6 \mathrm{ng} / \mathrm{L} 1.63[95 \% \mathrm{Cl}, 1.10-2.42])$.

Conclusions: High-sensitivity troponin I levels independently predict all-cause mortality in stable patients with COPD beyond well-established cardiovascular and respiratory prognostic indicators.

Waschki et al European Respiratory Journal 2018 52: OA2138.

\section{PS089 \\ Klinische Kasuistik - Progrediente Luftnot, rezidivierende Infekte, Ptosis bei einem 59-jährigen Patienten}

\section{Zsofia Kovacs; Thomas Wessendorf; Christian Taube}

Ruhrlandklinik, Westdeutsches Lungenzentrum, Universitätsklinikum Essen, Pneumologie, Essen, Germany

Einleitung: Die Tracheobronchomegalie Mounier-Kuhn (M-K-Syndrom) ist eine seltene Lungenerkrankung, die sich mit progredienter Luftnot und rezidiverenden Infekten meistens im Erwachsenalter präsentiert.
Fallvorstellung: Die Vorstellung des 59jährigen Patienten erfolgte zur Therapieverbessung und Evaluation der Möglichkeit einer Lungentransplantation. Der Patient beklagte eine seit Jahren bestehende progrediente Atemnot, aktuell bei leichter Belastung. Anamnestisch hervorheben sind in den letzten Jahren rezidivierende bronchopulmonale Infekte mit der Notwendigkeit mehrfacher Hospitalisationen.

Bei der körperlichen Untersuchung imponierten auskultatorisch ubiquitär grobblasige Rasselgeräusche, daneben fielen eine schwere Ptosis beider Augenlider sowie reponierbare Hernien beidseits auf.

Das CT-Thorax ließ mit typischem Aspekt einer auf 3.2cm dilatierten Trachea die Diagnsoe eines M-K Syndroms vermuten. Bronchoskopisch bestätigte sich der Befund mit typischer Kollapsibilität der Atemwege. Lungenfunktionanalytisch konnte eine schwere Obstruktion mit Überblähung gesichert werden. In der BGA fand sich unter 3 I $\mathrm{O} 2 / \mathrm{min}$ eine Hyperkapnie (pCO2 $52 \mathrm{mmHg}$ ).

Verlauf: Die bestehende NIV-Therapie wurde optimiert und die antiobstruktive Therapie intensiviert. Bei anhaltender eosinophiler Entzündung zur Ersparung der OCS-Therapie (NW mit DM, Osteoporose) wurde eine Therapie mit IL5-AK angedacht. Eine regelmäßige Antibiotika-Therapie erscheint zur Vermeidung häufiger Infekte sinnvoll. Der Patient wurde in der LTX-Konferenz vorgestellt, aber aufgrund der anatomischen Konstellation und sehr hohem perioperativen $\mathrm{Ri}$ siko abgelehnt.

Diskussion: Das M-K Syndrom ist eine seltene Erkrankung (ca. 300 publizierte Fälle), die sich in der Regel im 30.-40. Lebensjahr manifestiert. Es wurde von P. Mounier-Kuhn 1932 beschrieben. Die Diagnose kann bei typischer Anamnese nach radiologischen Kriterien gestellt werden. 3 Subtypen sind je nach Erweiterungsmuster bekannt. Pathophysiologisch besteht eine Atrophie der elastischen Fasern. Der Mechanismus ist unklar. Eine Ausdünnung der Muscularis mucosa, die Atrophie der longitudinalen Muskeln und elastischen Fasern sind pathognomonisch für das M-K-Syndrom. Die Tracheobronchomegalie kann ebenfalls ein Symptom verschiedener Bindegewebs-Krankheiten wie Ehlers-Danlos Syndrom, Cutis Laxa, Marfan-Syndrom Ataxia-telangiectasia, M. Bechterew, Kenny-Caffey-Syndrom oder, Brachmann-de Lange-Syndrom auftreten.

Gesichtsanomalien (Micrognathie, Mikrouvula, Ptosis) sind ebenfalls beschrieben worden. Der genaue Zusammenhang zwischen Ptosis und Tracheobronchomegalie ist nicht bekannt. Eine generalisierte Bindegewebsschwäche bei rezidiverenden Hernien könnte auch bei unserem Patienten bestehen, der vorliegende Fall konnte allerdings keiner bekannten Bindegewebe-Erkrankung zugeordnet werden.

\section{PS090}

Lungensport bei COPD-Patienten mit Schwerpunkt Kraftoder sensomotorisches Training verbessert die körperliche Leistungsfähigkeit

Marc Spielmanns'; Nicole Bott ${ }^{2}$; Klaus Baum ${ }^{3}$

'Zürcher RehaZentrum Wald, Pulmonale Rehabilitation, Wald, Switzerland; ${ }^{2}$ Luisenhospital Aachen und Deutsche Sporthochschule Köln, Pneumologie, Köln, Germany; ${ }^{3}$ Trainingsinstitut Prof. Dr. Baum und Deutsche Sporthochschule Köln, Physiologie, Köln, Germany

Zusammenfassung: Körperliches Training bei COPD-Patienten verbessert die Leistungsfähigkeit. Es ist aber unklar, ob durch eine Akzentuierung von Kraft- (KT) oder sensomotorischem Training (SM) innerhalb des Lungensports eine höhere Leistungssteigerung gegenüber einem niederschwelligen Training erreicht werden kann. In einer randomisierten kontrollierten Studie wurden die Ergebnisse dieser 
Trainingsformen in Bezug auf einen Zuwachs an Leistungsfähigkeit untersucht.

Methodik: Nach Randomisierung trainierten bis zum Ende der Studie (12 Wochen) 65 COPD-Patienten unterschiedlichen Schweregrads entweder $2 x$ wöchentlich über 60 Minuten in einer KT-, einer SM- oder in der Kontrollgruppe (KG). Die Endpunkte waren die Veränderungen im 6 Minuten-Gehtest (6-MWT), Sit-to-Stand Test (5-STST), Stair-ClimbTest (SCT) und in der isometrischen Kraftmessung (IsoK) zu Beginn ( $\mathrm{T}_{1}$ ) und am Ende ( $\left.\mathrm{T}_{3}\right)$ der Trainingsperiode.

Ergebnisse: In den anthropometrischen Daten zeigten sich keine signifikanten Veränderungen. Es konnten jedoch signifikante Verbesserungen in den unterschiedlichen Tests (s. Tabelle 1) gefunden werden. Angegeben sind die Differenzen von T1 zu T3 sowie die Standardabweichungen $\left(^{*}=\right.$ Differenz signifikant $\left.p<0,05\right)$ in den unterschiedlichen Trainingsgruppen (TG). Lediglich für die SM konnte in allen Leistungstests signifikante Verbesserungen gefunden werden. Im Intergruppenvergleich ergaben sich keine signifikanten Unterschiede.

$\begin{array}{llll} & \text { 5-STST in Sekunden } & \text { 6-MWT in Metern } & \text { SCT in Sekunden } \\ & \mathrm{T}_{3}-\mathrm{T}_{1}(\Delta 2 & \mathrm{T}_{3}-\mathrm{T}_{1}(\Delta 2) & \mathrm{T}_{3}-\mathrm{T}_{1}(\Delta 2) \\ \text { KG } & -1,79 \pm 2,99^{*} & 22,29 \pm 51,43 & 1,00 \pm 9,10^{*} \\ \text { KT } & -1,59 \pm 1,89^{*} & 29,14 \pm 34,63^{*} & -1,29 \pm 1,67^{*} \\ \text { SM } & -1,39 \pm 2,30^{*} & 32,14 \pm 39,32^{*} & -0,79 \pm 1,21^{*}\end{array}$

Schlussfolgerung: Durch die unterschiedlichen Trainingsformen konnten in den meisten Tests signifikante Leistungszuwächse gefunden werden. Leidglich für das SM waren in allen Leistungstests signifikante Verbesserungen feststellbar, weshalb das SM im Rahmen des Lungensports bezüglich des Gewinns an Leistungsfähigkeit am ehesten zu favorisieren ist.

\section{Prävention und Rehabilitation}

\section{PS091 \\ Training im ambulanten Lungensport steigert die Balancefähigkeit}

Marc Spielmanns'; Nicole Bott ${ }^{2}$; Klaus Baum ${ }^{3}$

'Zürcher RehaZentrum Wald, Pulmonale Rehabilitation, Wald, Switzerland; ${ }^{2}$ Luisenhospital Aachen und Deutsche Sporthochschule Köln, Pneumologie, Köln, Germany; ${ }^{3}$ Trainingsinstitut Prof. Dr. Baum und Deutsche Sporthochschule Köln, Physiologie, Köln, Germany

Einleitung: Die physische Dekonditionierung bei COPD-Patienten führt oft auch zur Einschränkung der Balancefähigkeit, welche mit einer erhöhten Sturzneigung verknüpft ist. Im Rahmen einer prospektiven, randomisierten, kontrollierten Studie wurde untersucht, inwiefern ein im Rahmen des ambulanten Lungensport eingeführtes, spezielles sensomotorisches Training (SM), positive Auswirkungen auf die Balancefähigkeit hat.

Methodik: Nach Randomisierung trainierten die Teilnehmer entweder in einer konventionellen Trainingsgruppe (KG), in einer Krafttrainingsgruppe (KT) oder im SM. Die Trainingsdauer betrug $2 \times 60$ Minuten pro Woche über insgesamt 12 Wochen. Die Endpunkte waren die Balancefähigkeit in der Fullerton Advanced Balance Scale (FAB) und der Berg-Balance Scale (BBS) im Intra- und Intergruppenvergleich.
Ergebnisse: Von 117 Teilnehmer konnten 65 die Studie regulär beenden und gingen in die Endauswertung ein. Die Zu T1 zeigte sich nur im BMI ein signifikanter Gruppenunterschied in den Baseline-Charakteristika. Die Scores sind in als Median und Interquartilsabstand dargestellt. Im Intragruppenvergleich $\mathrm{T}_{1}$ zu T3 (Abschlusszeitpunkt) konnte in allen drei Gruppen signifikante Verbesserungen im FAB erreicht werden. Lediglich die SM-Gruppe zeigte in der BBS keine signifikanten Verbesserungen. Zwischen den Gruppen gab es keinen signifikanten Unterschied.

* der p-Wert ist kleiner als das Signifikanzniveau mit $a=5 \%$
T1
T3
$\mathrm{T}_{3}-\mathrm{T}_{1}(\Delta \mathbf{2})$
p-Wert

KG

$\begin{array}{lrrrr}\text { BBS } & 52,00[5,00] & 54,00[3,00] & 0,00[2,00] & 0,005^{*} \\ \text { FAB } & 29,00[7,00] & 29,00[7,00] & 1,00[3,00] & 0,031^{*}\end{array}$

KT

$\begin{array}{lllll}\text { BBS } & 55,00[2,00] & 56,00[1,00] & 1,00[2,00] & 0,001^{*} \\ \text { FAB } & 34,00[10,00] & 36,00[6,00] & 1,50[3,00] & 0,002^{*}\end{array}$

SM

BBS $55,00[2,00] \quad 55,00[1,00] \quad 0,00[1,00] \quad 0,111$

$\mathrm{FAB} \quad 34,50[5,00] \quad 36,00[3,00] \quad 1,50[3,00] \quad 0,002^{*}$

Schlussfolgerung: Regelmäßiges Training im Rahmen des ambulanten Lungensports führt zu signifikanten Verbesserungen der Gleichgewichtsfähigkeit bei allen COPD-Patienten in diesem Kollektiv. Die Gruppen KT und SM konnten zwar größere Veränderungen erzielen als die Gruppe KG, jedoch muss in diesem Kontext die Frage offen bleiben, ob ein spezielles SM effektiver gegenüber einem KT ist.

\section{PS092 \\ Effekte des ambulanten Lungensports auf die Lebensqualität und Symptomatologie bei COPD-Patienten}

Marc Spielmanns'; Nicole Bott ${ }^{2}$; Klaus Baum ${ }^{3}$

'Zürcher RehaZentrum Wald, Pulmonale Rehabilitation, Wald, Switzerland; ${ }^{2}$ Luisenhospital Aachen und Deutsche Sporthochschule Köln, Pneumologie, Köln, Germany; ${ }^{3}$ Trainingsinstitut Prof. Dr. Baum und Deutsche Sporthochschule Köln, Physiologie, Köln, Germany

Die COPD ist durch einen zunehmenden Verlust an Lebensqualität (HRQL) und einer Zunahme an Dyspnoe gekennzeichnet. In einer prospektiven randomisierten Studie wurde der Effekt eines intensiven Kraft (KT)- oder koordinativen Trainings (SM) mit einem niederschwelligen Training (KG) im Rahmen des ambulanten Lungensports in Bezug auf die HRQL und Dyspnoesymptomatik der Teilnehmer (TN) verglichen.

Methodik: Der Lungensport wurde 2 x 60 Minuten (Min) pro Woche über insgesamt 12 Wochen durchgeführt. Neben dem körperlichen Training (45 Min) wurden Teile des COBRA-Schulungsprogramm vermittelt (15 Min). Zusätzlich trainierte die KT und SM im häuslichen Bereich. Die Endpunkte waren die Änderungen im Prä/Post Vergleich für St. George Respiratory Questionnaire (SGRQ) und COPD-Assessment-Test (CAT). 
Ergebnisse: Die Scores im CAT und SGRQ zu Beginn (T1) und zum Ende (T3) der Studie in den jeweiligen Gruppen sind in Tabelle 2 dargestellt. Die Daten werden als Median und Interquartilsabstand angegeben. Im CAT zeigte sich für keine Gruppe ein signifikanter Unterschied. Für die SM-Gruppe konnten sowohl im Summen-Score als auch in den Subscores des SGRQ signifikante Verbesserungen gefunden werden. In der KT verbesserte sich der Aktivitäts-Score signifikant. Im Intergruppenvergleich konnten keine signifikanten Unterschiede gefunden werden.

KG

\begin{tabular}{lllll}
\multicolumn{1}{c}{ T1 } & T3 & T3-T1 ( $\mathbf{( 1 2})$ & p-Wert \\
SGRQ Summe & $54,25[18,79]$ & $46,36[20,36]$ & $-1,60[26,18]$ & 0,205 \\
- Symptome & $58,46[36,05]$ & $58,57[32,15]$ & $-5,16[22,55]$ & 0,498 \\
- Aktivität & $72,29[17,03]$ & $66,31[25,87]$ & $0,00[14,74]$ & 0,438 \\
- Auswirkung & $41,02[24,07]$ & $34,03[22,32]$ & $-2,26[33,57]$ & 0,263 \\
CAT & $22,00[9,00]$ & $20,00[7,00]$ & $-2,00[6,00]$ & 0,552
\end{tabular}

KT

T1

$41,12[31,04]$

$\mathrm{T}_{3}-\mathrm{T}_{1}(\Delta \mathbf{2})$

p-Wert

- Symptome $\quad 45,70[33,07]$

$43,32[32,79]$

$-0,92[7,90]$

0,527

- Aktivität

$56,94[27,99]$

$56,90[32,24]$

$-1,89[21,87]$

0,685

- Auswirkung

$23,24[29,16]$

$23,84[31,38]$

$-5,47[11,58]$

$0,031^{*}$

CAT $17,50[15,00]$

$16,50[14,00]$

$-0,33[9,82]$

0,658

$-1,00[5,00]$

0,412

SM

\begin{tabular}{|c|c|c|c|c|}
\hline & $T_{1}$ & $T_{3}$ & $\mathrm{~T}_{3}-\mathrm{T}_{1}\left(\Delta_{2}\right)$ & p-Wert \\
\hline SGRQ Summe & $41,77[25,61]$ & $35,69[33,60]$ & $-6,50[12,19]$ & $0,006^{*}$ \\
\hline - Symptome & $51,69[28,73]$ & $53,87[39,69]$ & $-8,23[19,97]$ & $0,033^{*}$ \\
\hline - Aktivität & $57,00[24,05]$ & $47,30[28,12]$ & $-6,64[14,64]$ & $0,007^{*}$ \\
\hline - Auswirkung & $29,29[22,71]$ & $23,63[29,93]$ & $-3,88[10,96]$ & $0,018^{*}$ \\
\hline & $16,50[11,50]$ & $14,50[14,00]$ & $-1,50[9,00]$ & \\
\hline
\end{tabular}

Schlussfolgerung: Ein sensomotorisches Training im Rahmen des ambulanten Lungensports scheint die Lebensqualität gemessen im SGRQ zu steigern, wenngleich die Symptomatik, gemessen im CAT sich nicht wesentlich änderte.

\section{PS093 \\ Chronische Erkrankungen - „Silent Inflammation“ und das Potential körperlicher Aktivierung}

Ulrich Hamberger'; Martina M Hinner ${ }^{2}$

${ }^{1}$ Klinikum Landsberg /, Schmerzzentrum, Landsberg, Germany; ${ }^{2}$ Uhma International, Prevention, Scotch Plains, New Jersey, United States

Viele chronische Erkrankungen gehen mit einem inaktiven Lebensstil, begleitet von viszeralem Fettaufbau und Abbau von Muskulatur und Herzkreislauffunktion einher. (Booth, Roberts, Thyfault, Ruegsegger
\& Toedebusch, 2017)Die viszerale Fettansammlung zeigt sich häufig als Ursprungsort proinflammatorischer Zytokine. Deren dauerhaft erhöhtes Auftreten resultiert in einer chronischen subakuten systemischen Entzündung. (Pedersen, 2011) Diese sogenannte "Silent Inflammation", zusammen mit "Inflamm-Aging" und zunehmend auftretender Sarkopenie zeigen sich als Prädiktoren für kardiometabolische Erkrankungen, Multimorbidität, Gebrechlichkeit, und letztendlich erhöhtem Sterblichkeitsrisiko. (ARANGO-LOPERA, ARROYO, GUTIERREZ-ROBLEDO, PEREZ-ZEPEDA \& CESARI, 2013; Benatti \& Pedersen, 2015; Franceschi, Garagnani, Parini, Giuliani \& Santoro, 2018) (Stoever, Heber, Eichberg \& Brixius, 2017)Zweifelsohne kann körperliche Aktivität einen günstigen Einfluss auf kardiometabolische Risikofaktoren, Körperfett und Muskelmasse ausüben.Der aktive Muskel besitzt aber auch die Fähigkeit, Zytokine zu produzieren oder deren Produktion zu verursachen. Diese sog. Myokine besitzen u.a. das Potential inflammatorischen Prozessen einer Atherosklerose, (Libby, Ridker \& Maseri, 2002) oder denen neurodegenerativer Erkrankungen im ZNS entgegenzuwirken. (Erickson, Weinstein \& Lopez, 2012; Phillips, C. \& Fahimi, 2018; Yang et al., 2017) Aber auch die BDNF-Produktion im ZNS kann durch Muskelaktivität in Gang gesetzt werden, um so z.B. Lern - u. Gedächtnisfunktion des Hippocampus zu verbessern. (Fahimi et al., 2017) Myokine helfen bei Mobilisation und Verbrennung von Fetten und Zucker, bei der Verbesserung von Endothelfunktion und Angiogenese, veranlassen Makrophagen zur Reduktion inflammatorischer, und Produktion antiinflammatorischer Zytokine, oder den Muskel zur Hypertrophie. (Benatti \& Pedersen, 2015) Ausdauertraining entfaltet schon in moderater Dosis kardiometabolisch günstige und auch antiinflammatorische Wirksamkeit. Neben der Myokin Ausschüttung wird dies auch über den Vagus bewirkt, der ebenso über moderates Ausdauertraining in Gang gesetzt werden kann. (Pavlov \& Tracey, 2012; Shiotani, Umegaki, Tanaka, Kimura \& Ando, 2009) Muskelaufbautraining erfordert hingegen grundsätzlich eine hohe Intensität, um über Stresshormone und auch proinflammatorische Zytokine einen trainingswirksamen Reiz zu bewirken und Muskelabbauprozessen entgegen zu wirken. (Gomez-Cabrera, Viña \& Ji, 2016) Damit dieser Balanceakt mit dem Immunsystem Richtung anabol wirkt, sind Trainings- und Regenerationsdauer von entscheidender Bedeutung. (Pesta \& Roden, 2017; Phillips, S. M., 2009) Die Messung der Herzraten- Variabilität zeigt sich hierbei als geignetes Instrument, um psychische und körperliche Beanspruchung zu erkennen. (Sammito \& Böckelmann, 2016) Die nat. Empfehlungen für Bewegung $u$. Bewegungsförderung bieten eine gute Orientierung zu Art und Dosierung körperlicher Aktivierung. (Rütten et al., 2016).

ARANGO-LOPERA, V. E., ARROYO, P., GUTIERREZ-ROBLEDO, L. M., PEREZ-ZEPEDA, M. U. \& CESARI, M. (2013). MORTALITY AS AN ADVERSE OUTCOME OF SARCOPENIA. The journal of nutrition, health \& aging, 17, 259-262. https://doi.org/10.1007/s12603-012-0434-o

Bano, G., Trevisan, C., Carraro, S., Solmi, M., Luchini, C., Stubbs, B. et al. (2017). Inflammation and sarcopenia: A systematic review and meta-analysis. Maturitas, 96, 10-15. https://doi.org/10.1016/j.maturitas.2016.11.006

Benatti, F. B. \& Pedersen, B. K. (2015). Exercise as an anti-inflammatory therapy for rheumatic diseases-myokine regulation. Nature reviews. Rheumatology, 11, 86-97. https://doi.org/10.1038/nrrheum.2014.193

Booth, F. W., Roberts, C. K., Thyfault, J. P., Ruegsegger, G. N. \& Toedebusch, R. G. (2017). Role of Inactivity in Chronic Diseases: Evolutionary Insight and Pathophysiological Mechanisms. Physiological reviews, 97, 1351-1402. https://doi.org/10.1152/physrev.00019.2016

Erickson, K. I., Weinstein, A. M. \& Lopez, O. L. (2012). Physical Activity, Brain Plasticity, and Alzheimer's Disease. Archives of medical research, 43, 615-621. https://doi.org/10.1016/j.arcmed.2012.09.008 
Fahimi, A., Baktir, M. A., Moghadam, S., Mojabi, F. S., Sumanth, K., McNerney, M. W. et al. (2017). Physical exercise induces structural alterations in the hippocampal astrocytes: exploring the role of BDNF-TrkB signaling. Brain Structure and Function, 222, 1797-1808. https://doi. org/10.1007/s00429-016-1308-8

Franceschi, C., Garagnani, P., Parini, P., Giuliani, C. \& Santoro, A. (2018). Inflammaging: a new immune-metabolic viewpoint for age-related diseases. Nature reviews. Endocrinology, 14, 576-590. https://doi. org/10.1038/s41574-018-0059-4

Gomez-Cabrera, M. C., Viña, J. \& Ji, L. L. (2016). Role of Redox Signaling and Inflammation in Skeletal Muscle Adaptations to Training. Antioxidants, 5. https://doi.org/10.3390/antiox5040048

Libby, P., Ridker, P. M. \& Maseri, A. (2002). Inflammation and Atherosclerosis. Circulation, 105, 1135-1143. https://doi.org/10.1161/hc0902.104353

Pavlov, V. A. \& Tracey, K. J. (2012). The vagus nerve and the inflammatory reflex--linking immunity and metabolism. Nature reviews. Endocrinology, 8, 743-754. https://doi.org/10.1038/nrendo.2012.189

Pedersen, B. K. (2011). Exercise-induced myokines and their role in chronic diseases. Brain, behavior, and immunity, 25, 811-816. https:// doi.org/10.1016/j.bbi.2011.02.010

Pesta, D. \& Roden, M. (2017). The Janus Head of Oxidative Stress in Metabolic Diseases and During Physical Exercise. Current Diabetes Reports, 17, 41. https://doi.org/10.1007/s11892-017-0867-2

Phillips, C. \& Fahimi, A. (2018). Immune and Neuroprotective Effects of Physical Activity on the Brain in Depression. Frontiers in Neuroscience, 12, 498. https://doi.org/10.3389/fnins.2018.00498

Phillips, S. M. (2009). Physiologic and molecular bases of muscle hypertrophy and atrophy: impact of resistance exercise on human skeletal muscle (protein and exercise dose effects). Applied physiology, nutrition, and metabolism = Physiologie appliquee, nutrition et metabolisme, 34, 403-410. https://doi.org/10.1139/Ho9-042

Rütten, A., Pfeifer, K., Banzer, W., Ferrari, N., Füzéki, E., Geidl, W. et al. (2016). National Recommendations for Physical Activity and Physical Activity Promotion. Erlangen: FAU University Press.

Sammito, S. \& Böckelmann, I. (2016). Factors influencing heart rate variability. International Cardiovascular Forum Journal, 6. https://doi. org/10.17987/icf.v6io.242

Shiotani, H., Umegaki, Y., Tanaka, M., Kimura, M. \& Ando, H. (2009). Effects of aerobic exercise on the circadian rhythm of heart rate and blood pressure. Chronobiology international, 26, 1636-1646. https:// doi.org/10.3109/07420520903553443

Stoever, K., Heber, A., Eichberg, S. \& Brixius, K. (2017). Sarcopenia and Predictors of Skeletal Muscle Mass in Elderly Men With and Without Obesity. Gerontology and geriatric medicine, 3. https://doi. org/10.1177/2333721417713637

Yang, J., Cao, R. Y., Gao, R., Mi, Q., Dai, Q. \& Zhu, F. (2017). Physical Exercise Is a Potential "Medicine" for Atherosclerosis. In J. Xiao (Ed.), Exercise for Cardiovascular Disease Prevention and Treatment. From Molecular to Clinical, Part 1 (Advances in Experimental Medicine and Biology, vol. 999, pp. 269-286). Singapore: Springer Singapore. https://doi. org/10.1007/978-981-10-4307-9_15

\section{PS094}

Jugendliche mit Adipositas permagna, Typ2-Diabetes und weiteren Folgeerkrankungen vor letzter Option der bariatrischen Chirurgie - Beispielhafter Fallbericht und Therapiekonzept

Richard Eyermann 1,2

'Dr. Eyermann München, Kinder- und Jugendmedizin, Kinderkardiologie, Sportmedizin,München,Germany; ${ }^{2}$ Klinik Schönsicht Berchtesgaden, Rehabilitation für Kinder und Jugendliche, AHB, Kind-Mutter / Vater-Rehabilitation, München,Germany

Bariatrische Operationen ermöglichen zwar relativ rasche u. vs. konservativer Therapie häufig höhere Gewichtsabnahme, bergen aber relevante peri- u. postop. Risiken u. werden im Kindes- u. Jugendalter i.d.R. abgelehnt (AGA, DAG).

\section{Fallbericht:}

Diagnosen:

Extreme Adipositas

Dm2

Arterielle HTN

Dyslipidämie

Hyperurikämie

NASH

Sekundäre Amenorrhoe

Extreme Genua valga

Senk-Spreiz-Füße

15 9/12-jähriges Mädchen, extrem adipös, Acanthosis nigricans, Striae dist. rubrae, Hepar 1QF, KL 159,5 cm, KG 154,4 kg, BMl 60.69kg/m2 (BMISDS 4.62; >99.5 Pc.). Taillenumfang $134 \mathrm{~cm}$, Bauchumfang $157 \mathrm{~cm}$. Bei angenommenem Normalgewicht von @ 54 kg lag bei Aufnahme ein Übergewicht von $100 \mathrm{~kg}$ vor. Extreme Genua valga (Op-Indikation).

Im Vorfeld zahlreiche frustrane ambulante Therapieversuche. Bariatrische Chirurgie bei höchstem Gesundheitsrisiko bereits universitär in Austria geplant.

Bei initialer Dm2-Therapie mit $2 \mathrm{~g}$ Metformin, u. Insulin (33 IE Basalinsulin $\left(\right.$ Lantus $\left.{ }^{\circledast}\right)$ sowie $>5$ olE Actrapid auf $3 E D$ ) konnte durch Gewichtsreduktion von $34 \mathrm{~kg}$ schon sign. Verbesserung erreicht werden. Insulintherapie beendet nach 6 Wo. bei konstant gutem BZ, Metformin nach 10 Wo..HbA1c anfangs $>8 \%$, bei Kontrollen rückläufig (nach 14 Tagen 7,4\%, nach 4 Wo. 6,7 \%, nach 8 Wo. 6,0\%). In letzten BZ-TP keine Hyperglykämien.

Homa-IR zunehmend reduziert, mit 3,5 noch etwas erhöht (Insulinresistenz).

Dyslipidämie, erhöhte Leberwerte, Hyperurikämie deutlich rückläufig - einst Signale erhöhten metabolischen Risikos bzw. Fettleberentwicklung. HTN von ehemals zer-Kombination jetzt nur noch mit ACEI einreguliert.

Erfreulich auch Ende der Amenorrhoe.

Die Jugendliche nimmt am umfangreichen Sportprogramm bestehend aus Ausdauersport (Joggen, Wandern, Schwimmtraining), Haltungs- u. Krafttraining u. Spielsportarten teil, erhält kalorienreduzierte Mischkost 1500 kcal/Tag, Diätschulungen, Diätkochkurs Einkaufstraining, Verhaltenstherapie.

Diskussion u. Fazit: In 18 Wo. gelang durch erste Gewichtsabnahme von $34 \mathrm{~kg}$ sign. Reduktion des stark erhöhten kardiometabolischen Risikos, v.a. Beseitigung insulinpflichtigen Dm2 sowie Intervention 
psychischer Störungen mit Steigerung QoL u. Selbstwertgefühl. Gebessert auch Genua valga - z.Z. keine Op-Indikation mehr.

Frage Nachhaltigkeit: Die Jugendliche wurde über Weihnachten u. Neujahr 4 Wo. beurlaubt. Zu Hause konnte sie ihr KG halten - und dies über die Feiertage !!!

Der BZ-Memory vom Monat zwischen den beiden stationären Maßnahmen zeigte Normoglykämie.

Nach weiteren 8 Wochen Reha gelang weitere Reduktion von KG $(15,8 \mathrm{~kg})$ sowie kardiometabolischer Risikofaktoren, Transaminasen normal. Keine Proteinurie.

BMI 37,37 kg/m2 (99,91. Perzentile; BMI-SDS: + 3,13).

Die Jugendliche wird erlernten gesunden Lebensstil weiter praktizieren $u$. weiterverbreiten.

Ausreichend lange stationäre Reha kann in schweren Adipositasfällen das fatale metabolische Syndrom signifikant intervenieren $u$. bariatrische Chirurgie als letzte Option verhindern.

\section{PS095}

Auswirkungen eines 3-wöchigen Interventionsprogramms auf die Körperzusammensetzung bei adipösen Kindern und Jugendlichen gemessen mit bioelektrischer Impedanzanalyse (BIA)

Richard Eyermann', ${ }^{1}$; H. Langhof ${ }^{2}$; M. Kaestner ${ }^{3}$; H. Schulz ${ }^{3}$; S. Öhmichen ${ }^{3}$

'Dr. Eyermann München, Kinder- und Jugendmedizin, Kinderkardiologie, Sportmedizin, München, Germany; ${ }_{i}^{4}$ Klinik Schönsicht Berchtesgaden, Rehabilitation für Kinder und Jugendliche, AHB, Kind-Mutter / Vater-Rehabilitation, München,Germany; ${ }^{2}$ Klinik Schönsicht Berchtesgaden, Rehabilitation für Kinder und Jugendliche, AHB, Kind-Mutter/Vater-Rehabilitation, Berchtesgaden, Germany; ${ }^{3} \mathrm{TU}$ Chemnitz, Sportwissenschaft, Chemnitz, Germany; ${ }^{3} \mathrm{TU}$ Chemnitz, Sportwissenschaft, Chemnitz, Germany; ${ }^{3} \mathrm{TU}$ Chemnitz, Sportwissenschaft, Chemnitz, Germany

Problem: Adipositas nach WHO eines der größten Gesundheitsprobleme des 21. Jh..

Ziel: Ziel war die Wirksamkeit der sportlichen Intervention in der Reha zu überprüfen, ob diese zu der gewünschten Gewichtsreduktion durch Verlust von Körperfett führt u. ob in einem Interventionszeitraum von 3 Wo. schon sign. Ergebnisse erzielt werden können.

Methodik: 34 Kinder u. Jugendliche stationär in Reha multimodal behandelt, 20 w. u. 14 m., im Alter von $10-17$ Jahren, BMI 34,6kg/m2 (MW) \pm 6.9 (SD) bzw. $33,3 \mathrm{~kg} / \mathrm{m} 2$ (MW) $\pm 7,4$ (SD).

Einschlußkriterien: für mindestens 4 Wo. Patient, Diagnose Adipositas, ärztl. Sporterlaubnis, zwischen 10-18 Jahren. Ausschlusskriterien: Medikamente, die Flüssigkeitshaushalt beeinflussen, Infekt (akut während letzter 14 Tage), metallische Implantate (OP-induziert), Schwangerschaft, Herzschrittmacher, Defibrillator.

Messungen mit 2 BIA-Geräten, MALTRON Körperfett-Analysegerät BF906 u. BIA-Gerät „DENNER Systemtechnik“ .

Daten sowohl deskriptiv als auch inferenzstatistisch ausgewertet (Statistikprogramm IBM SPSS 21).

Ergebnisse u. Diskussion: Studie untersuchte kurzfristige Auswirkungen von körperlicher Bewegung auf die Körperzusammensetzung. Verwendetes Studiendesign ist durchaus gut gewählt $u$. bietet gute Grundlage um die Fragen zu beantworten. Allerdings bleiben hier die Langzeitwirkungen außen vor, welche gerade im Bereich der kindlichen u. jugendlichen Adipositas von großer Bedeutung sind, wie Folgeerkrankungen. Trotz des eher geringen Zeitraums zeigt die Studie die Effektivität solcher Interventionsprogramme u. konnte dem Ziel der Gewichtsreduktion durch Körperfettabnahme gerecht werden.

Körpergewicht: Der MW lag beim Prae-Test bei $89,74 \pm 18,46 \mathrm{~kg} \mathrm{u}$. bei der Messung nach 3 Wo. bei $86,05 \pm 17,58 \mathrm{~kg}$ bei den Mädchen, bei den Jungen MW 97,34 $\pm 35,81 \mathrm{~kg}$ bzW. 92,21 $\pm 33,33 \mathrm{~kg}$.

Körperfett: Fettanteil sank von $39,13 \pm 3,58 \%$ auf $37,34 \pm 3,48 \%$ bei den Mädchen, bei den Jungen von $33,61 \pm 9,38 \%$ auf $30,89 \pm 10,18 \%$.

Muskelmasse: Zunahme um 0,29\% bei den Mädchen bzw. 0,33\% bei den Jungen.

Körperwasser: Zunahme bei Mädchen u. Jungen.

Body Cell Mass: Steigerung um 2,73\% bei den Mädchen u. 2,56\% bei den Jungen.

Resistanz(R) u. Reaktanz(Xc): Veränderungen waren bei keinem der beiden Geschlechter signifikant.

Phasenwinkel: Veränderung nicht signifikant.

Der ebenfalls untersuchte Gerätevergleich zeigt auf, dass die verwendeten BIA-Geräte gleich messen. Sie haben dasselbe Funktionsprinzip. Für zukünftige Studien besteht hier die Option, weitere Meßgeräte mit einzubeziehen $u$. diese untereinander zu vergleichen, z.B. sind Vergleiche zwischen BIA, MRT u. DEXA möglich, zudem kann man diese mit den „einfachen u. kostengünstigen“ Varianten, wie z.B. Hautfaltenmessung vergleichen.

Konklusion: Die angewandten sportlichen Interventionen in der Reha erreichen die positiven u. erwünschten Wirkungen u. sollten als wirksame Maßnahme gegen die Volkskrankheit Adipositas beibehalten u. etabliert werden. Studie trägt zu neuen Erkenntnissen zur Adipositasproblematik bei.

\section{PS096}

Ergebnisse eines $6 \mathrm{~min}$-Laufs bei adipösen sowie normalgewichtigen Kindern und Jugendlichen an der Klinik Schönsicht Berchtesgaden

Richard Eyermann ${ }^{1}$,; H. Langhof ${ }^{2}$; L. Heinrichs ${ }^{3}$; H.-V. Ulmer ${ }^{3}$

'Dr. Eyermann München, Kinder- und Jugendmedizin, Kinderkardiologie, Sportmedizin, München, Germany; ${ }^{4} K$ Klinik Schönsicht Berchtesgaden, Rehabilitation für Kinder und Jugendliche, AHB, Kind-Mutter / Vater-Rehabilitation, München, Germany; ${ }^{2}$ Klinik Schönsicht Berchtesgaden, Rehabilitation für Kinder und Jugendliche, AHB, Kind-Mutter/Vater-Rehabilitation, Berchtesgaden, Germany; ${ }^{3}$ Johannes Gutenberg-Universität Mainz, Sportwissenschaft, Mainz, Germany; ${ }^{3}$ Johannes Gutenberg-Universität Mainz, Sportwissenschaft, Mainz, Germany

Problemstellung: Leistungsdiagnosen dienen zur Einordnung des aktuellen Leistungszustandes. Dies ist besonders wichtig an Kliniken, da hier anhand von Anfangs- und Enddiagnosen Schlüsse über den Erfolg des Klinikaufenthaltes gezogen werden. Bestandteil der Diagnose ist oftmals auch ein Sporttest.

Methodik: In der vorliegenden Studie wurden Daten (Januar 2017 bis Mai 2018, n=298) einer Klinik für Kinder und Jugendliche und Kinder 
zum 6min-Lauf, der Bestandteil der Leistungsdiagnose ist, ausgewertet. Dabei wurde die Anfangsleistung mit der Endlaufleistung verglichen, der BMI mit der Anfangs. Und Endlaufleistung korreliert, der Retest bezüglich der Reliabilität ausgewertet, die sportliche Aktivität des vorangegangenen Jahres mit der Anfangslaufleistung korreliert, sowie die Standardisierung des $6 \mathrm{~min}$-Laufs beschrieben.

Ergebnisse und Diskussion: Die Ergebnisse konnten eine Steigerung der Laufleistung mit einer durchschnittlichen Verbesserung von $81 \mathrm{~m}$ bei den Jungen bzw. $108 \mathrm{~m}$ bei den Mädchen belegen. Während eine hohe Korrelation zwischen Retest und Laufleistung festgestellt werden konnte, besteht nur eine geringe Korrelation zwischen BMI und Laufleistung sowie zwischen der sportlichen Aktivität im vorangegangenen Jahr und der Anfangslaufleistung. Bezüglich der Standardisierung des 6min-Laufs konnte ein hoher Durchführungsstandard festgestellt werden, während die Teststandards an sich vereinzelt Mängel aufwiesen.

Konklusion: Für die Praxis kann geschlussfolgert werden, dass der $6 \mathrm{~min}$-Lauf durchaus ein Mittel zur Leistungsdiagnose darstellt. Dennoch ist zu berücksichtigen, dass er oftmals zur Analyse der aeroben Ausdauerleistung durchgeführt wird. Dies kann aber nur unter Berücksichtigung der Gütekriterien erfolgen, die neben der Standardisierung auch die Validität umfassen. Diese kann jedoch erst anhand eines weiteren Kriteriums, neben der Laufleistung, wie beispielsweise der Schwimmleistung, festgestellt werden und erfordert folglich zusätzliche Daten zur Auswertung.

\section{PS097 \\ Sport- und Bewegungstherapie bei adipösen Kindern und Jugendlichen}

Richard Eyermann ${ }^{1}{ }^{4} ;$ H. Langhof ${ }^{2} ;$ K. Thieß ${ }^{2} ;$ Z. Bieniek $^{2}$

'Dr. Eyermann München, Kinder- und Jugendmedizin, Kinderkardiologie, Sportmedizin, München, Germany; ${ }^{4}$ Klinik Schönsicht Berchtesgaden, Rehabilitation für Kinder und Jugendliche, AHB, Kind-Mutter / Vater-Rehabilitation, München, Germany; ${ }^{2}$ Klinik Schönsicht Berchtesgaden, Rehabilitation für Kinder und Jugendliche, AHB, Kind-Mutter/Vater-Rehabilitation, Berchtesgaden, Germany; ${ }^{2}$ Klinik Schönsicht Berchtesgaden, Rehabilitation für Kinder und Jugendliche, AHB, Kind-Mutter/Vater-Rehabilitation, Berchtesgaden, Germany; ${ }^{2}$ Klinik Schönsicht Berchtesgaden, Rehabilitation für Kinder und Jugendliche, AHB, Kind-Mutter/Vater-Rehabilitation, Berchtesgaden, Germany

Problemstellung: Adipositas global endemisch, nimmt weiter $\mathrm{zu}$, muss v.a. aufgrund gravierender Folgemorbidität u. -mortalität interveniert werden. In BRD ca.15\% Kinder u. Jugendliche übergewichtig (AGA), 6,3\% davon adipös, mit Zunahme v.a. extremer Adipositas (KIGGS Daten).

Kardiometabolische Risikofaktoren persistieren, werden ins Erwachsenenalter mitgenommen (Tracking-Phänomen).

Methodik: Studie zur Wirksamkeit von Sport u. Bewegungstherapie bei adipösen Kindern u. Jugendlichen bei min. 4-6 wö. Rehaaufenthalt. Patientengut des gesamten Jahres 2015 einer Rehaklinik analysiert.

Ergebnisse: Zur Beurteilung der Leistungsfähigkeit nach Anreise 6MWD. Daraufhin Patienten in Leistungsgruppen eingeteilt, Wechsel im weiteren Rehaverlauf möglich. Weiterer 6MWD in Abreisewoche.

Übergeordnetes Ziel: Ziel Sporttherapie ist Teufelskreis aus verringerter Leistungsfähigkeit, Misserfolgserlebnissen, Frusttrationen verbunden mit Ängsten, Rückzug zur Inaktivität zu durchbrechen. Umfangreiches Sportprogramm angeboten.
Funktionelle u. motorische Ziele der Sporttherapie:

- Verbesserung motorischer Fähigkeit (Ausdauer, Koordination u. Kraft)

- Steigerung Energieumsatz durch körperliche Aktivitäten u. Erhöhung bzw. Erhalt des Ruheumsatzes trotz Gewichtsabnahme

- Verletzungsprophylaxe

\section{Psychosoziale Ziele:}

- Positives Erleben von Sport/körperlicher Aktivität

- Abbau von körper- u. bewegungsbezogenen Ängsten

- Schulung von Selbst- u. Körperwahrnehmung u. Selbstkontrolle

- Förderung sozialer Interaktion

- Therapiemotivation steigern

- Schulung von Selbstwirksamkeit ...u.s.w.

Zur Umsetzung dieser Ziele folgende sporttherapeutische Einheiten unterrichtet/angeboten:

- Sportspiele (Spiel u. Spaß im Vordergrund, Regelverständnis)

- Ausdauersport (Verbesserung Ausdauerleistungsfähigkeit)

- Schwimmen (Verbesserung Schwimmtechnik, Steigerung der Ausdauerleistungsfähigkeit)

- Geführte Wanderung (Verbesserung Ausdauer, Naturerlebnis)

- Haltung- u. Krafteinheit (Ausgleich muskulärer Dysbalancen)

- Wassergymnastik (Kraft- u. Koordinationsschulung)

- Bouldern

- Skilanglauf

- Boxen

- Adipositasschulung bezogen auf Sporttheorie (Energiebilanz, Bewegungspyramide, Puls, Gelenkschutz, Sportprogramm für zuhause).

Konklusion: Insgesamt 10h Sporttherapie/Wo. zur Anwendung. Zusätzlich weitere $6 \mathrm{~h}$ im Rahmen der Gruppenpädagogik.

Evident Verbesserung der Ausdauerleistung beim 6MWD vor Abreise: 2015 um @12,1 \%. Auch kleine Verbesserungen motorischer Leistungen vom Therapeuten wahrgenommen u. belobigt. Die mit Steigerung körperlicher Leistungsfähigkeit verbundene Verbesserung Selbstwert- und Lebensgefühls ist wesentliche Motivationshilfe, um auch für die Zukunft gewissen Trainingsfleiß zu etablieren.

Sport- u. Bewegungstherapie wesentlicher Bestandteil der Adipositasbehandlung, in Akutbehandlung, v.a. aber in Nachhaltigkeit, in Erziehung zu lebenslangem gesunden Lebensstil.

\section{PS098}

Extrem zeiteffizientes Intervallausdauertraining verbessert kardiometabolisches Risikoprofil und Inflammationsstatus bei Adipösen mit erhöhtem Risiko für das Metabolische Syndrom: Eine randomisiert-kontrollierte Pilotstudie

Dejan Reljic'; Fabienne Frenk²; Maike Tobschall²; Melanie Klaußner²; Hans Joachim Herrmann ${ }^{2}$; Markus F. Neurath ${ }^{3}$; Yurdagül Zopf ${ }^{2}$

'Universitätsklinikum Erlangen - Medizinische Klinik ', Medizinische Klinik 1, Erlangen, Germany; ${ }^{2}$ Universitätsklinikum Erlangen - Medizinische Klinik ${ }^{1}$, Hector-Center für Ernährung, Bewegung und Sport, Erlangen, Germany; ${ }^{3}$ Universitätsklinikum Erlangen - Medizinische Klinik', Gastroenterologie, Pneumologie und Endokrinologie, Erlangen, Germany

Hintergrund: Adipositas ist mit einem erhöhten Risiko für Diabetes, Herz-Kreislauf- und bestimmte Krebserkrankungen assoziiert. Liegen weitere Risikofaktoren (z.B. Hypertonie oder Hypercholesterinämie) vor, wird das Risiko für Folgeerkrankungen nochmals potenziert. Dabei spielen Entzündungsprozesse, die durch das Fettgewebe induziert werden, eine zentrale Rolle. Regelmäßige körperliche Aktivität kann das kardiometabolische Risiko nachweislich positiv beeinflussen. Die allgemeinen Bewegungsempfehlungen der WHO (150 Min. 
Aktivität/Woche) werden vom Großteil der Bevölkerung, insbesondere von Adipösen, jedoch nicht meist erreicht. „Zeitmangel“ zählt zu den häufigsten Gründen für unzureichende körperliche Aktivität. Ziel dieser Studie war es, die Effekte eines extrem zeitsparenden, hochintensiven Intervallausdauertrainings (HIIT, Zeitaufwand: 14 Min/Einheit) auf das kardiometabolische Risikoprofil und den Inflammationsstatus bei Adipösen zu untersuchen.

Methoden: 42 adipöse Männer und Frauen (48 \pm 12 Jahre, BMI: 38.2 \pm 7.7 , Körperfett: $44.3 \pm 7.2 \%)$ wurden randomisiert einer Trainings- $(\mathrm{HI}-$ IT-Gruppe, $n=20$ ) oder Kontrollgruppe ohne Training (CON, $n=22$ ) zugeteilt. Beide Gruppen erhielten eine Ernährungsberatung. HIIT wurde auf Radergometern (5X1 Min bei 85-95\% der maximalen Herzfrequenz) $2 x /$ Woche über 12 Wochen durchgeführt. Vor und nach der Studie wurden der Inflammationsstatus (C-reaktives Protein [CRP] und hochsensitives CRP [hsCRP] im Serum) und das kardiometabolische Risikoprofil (Metabolischer Syndrom Z-Score, MetS) bestimmt. MetS wurde anhand von Nüchtern-Glukose, Triglyzeriden, HDL-Cholesterin, mittlerem arteriellem Blutdruck und Taillenumfang berechnet.

Ergebnisse: HIIT wurde gut toleriert und es traten keine unerwünschten Vorfälle auf. Die Compliance betrug 92\%. Beide Gruppen reduzierten das Körpergewicht signifikant (HIIT: $-2.5 \mathrm{~kg}, \mathrm{P}<0.05 ; \mathrm{CON}:-2.8 \mathrm{~kg}$, $\mathrm{P}<0.01$ ). In der HIIT-Gruppe (Baseline-Werte: CRP: $5.7 \pm 5.4 \mathrm{mg} / \mathrm{l}$, hsCRP: $5.1 \pm 5.5 \mathrm{mg} / \mathrm{l}$, MetS: $2.4 \pm 4.3$ ) zeigten sich signifikante Abnahmen des CRP $(-1.6 \mathrm{mg} / \mathrm{l}, \mathrm{P}<0.05)$, hsCRP $(-1.9 \mathrm{mg} / \mathrm{l}, \mathrm{P}<0.05)$ und MetS $(1.4 \mathrm{Ein}-$ heiten, $\mathrm{P}<0.01$ ). In der $\mathrm{CON}$ veränderten sich diese Parameter nicht gegenüber den Baseline-Werten (CRP: $3.0 \pm 1.7 \mathrm{mg} / \mathrm{l}$, hsCRP: $2.4 \pm 1.4$ $\mathrm{mg} / \mathrm{l}$, MetS: 1.1 \pm 2.7 ).

Schlussfolgerung: Die vorliegenden Ergebnisse weisen darauf hin, dass mit dieser innovativen Trainingsform bereits ein Zeitaufwand von < 30 Min/Woche (ein Fünftel der WHO-Empfehlung) ausreichen kann, um bei Adipösen mit erhöhtem Risiko für das Metabolische Syndrom klinisch relevante Verbesserungen des Inflammationsstatus und kardiometabolischen Risikoprofils zu induzieren, die vergleichbar mit pharmakologischen Interventionen sind. Die hohe Adhärenz weist zudem auf eine sehr gute Verträglichkeit und Akzeptanz von HIIT bei Adipösen hin. Diese Daten untermauern die therapeutische Bedeutung und positiven Effekte von körperlicher Aktivität bei Adipositas, die weit über die reine Gewichtsreduktion durch Kalorienrestriktion hinausgehen

\section{PS099}

\section{Shisha smoking and risk of cardiovascular disease}

Radwan Al Ali'; Viktoria Schwarz² Lama Katmeh $^{2}$; Christian Werner ${ }^{2}$; Felix Mahfoud $^{2}$; Michael Böhm²

'Universitätsklinikum des Saarlandes, Kardiologie, Saarbrücken, Germany, ${ }^{2}$ Universitätsklinikum des Saarlandes, Homburg, Kardiologie, Homburg, Germany

Background and aims: The associated harm of tobacco smoking on the cardiovascular system is worldwide well known for cigarettes. But there are still only few studies about this effect for shisha smoking, which is becoming more prevalent globally, particularly among younger populations and believed to be "less harmful". The aim of this study is to investigate the relation of shisha smoking to some of cardiovascular risk parameters compared to cigarette smoking among adults in a general population.

Methods: A cross-sectional study based on Data from a cardiovascular risk factors survey conducted by University of Aleppo in cooperation with Syrian Centre for Tobacco Studies in 2006 in Aleppo city. A total of 806 participants were included, who provided demographic, beha- vioral, and smoking-related information, as well as measurements of weight, height, blood pressure, and heart rate. Fasting blood samples were obtained to analyze C-reactive protein, glucose, triglyceride, total cholesterol, LDL- and HDL-cholesterol. The correlation between smoking and the studied parameters was assessed using multivariate linear regression analyses adjusted for potential confounders (age, gender, BMI, physical activity).

Results: Daily shisha smoking was associated with an increased BMI $(p=0.009)$, whereas an opposite association was observed with daily cigarette smoking $(p<0.001)$. Daily cigarette smoking independently correlated with higher levels of LDL-cholesterol $(p=0.003)$ and CRP ( $p$ $<0.001$ ), while shisha smoking correlated with higher levels of triglyceride $(p=0.012)$. Both shisha und cigarette smoking correlated with lower HDL-cholesterol levels ( $p=0.012$ and $p=0.004$ respectively). Unlike cigarette smoking, daily shisha smoking was associated with increased heart rate $(p<0.001)$. Blood pressure tended to be increased among daily shisha smokers and to be decreased among daily cigarette smokers.

Conclusion: The results of this study show that shisha smoking is associated with cardiovascular biomarkers abnormalities, suggesting to be as harmful for cardiovascular health as Cigarette smoking.

\section{PS100}

Baseline Medical Conditions Do Not Impact the Efficacy and Safety of the Adjuvanted Recombinant Zoster Vaccine: a Posthoc Analysis of Pooled Data from Two Parallel Randomized Trials

Johannes Hain'; Lidia Oostvogels ${ }^{2}$; Thomas Heinemann ${ }^{17}$; Robert Johnson ${ }^{3}$; Myron Levin ${ }^{4}$; Janet McElhaney ${ }^{5}$; Peter Van den Steen ${ }^{18}$; Toufik Zahaf ${ }^{19}$; Alemnew Dagnew ${ }^{17}$; Roman Chlibek ${ }^{6}$; Javier Diez Domingo ${ }^{7}$; Iris Gorfinkel $^{8}$; Caroline Herve ${ }^{20}$; Shinn-Jang Hwang ${ }^{9}$; Hideyuki Ikematsu ${ }^{10}$; George Kalema ${ }^{11} ;$ Himal Lal ${ }^{17}$; Shelly McNeil' ${ }^{12}$; Tomas Mrkvan ${ }^{18}$; Karlis Pauksen ${ }^{13}$; Jan Smetana ${ }^{6}$; Daisuke Watanabe ${ }^{14}$; Lily Weckx ${ }^{15}$; Anthony Cunningham ${ }^{16}$

'GlaxoSmithKline GmbH \& Co. KG, Medizinischer Fachbereich Impfstoffe, München, Germany; ${ }^{2}$ GlaxoSmithKline, Clinical and Epidemiology, Wavre, Belgium; ${ }^{3}$ University of Bristol, Faculty of Health Sciences, Bristol, United Kingdom; ${ }^{4}$ University of Colorado, Departments of Pediatrics and Medicine, Aurora, United States; ${ }^{5}$ Health Sciences North Research Institute, Health Sciences North Research Institute, Sudbury, Canada; ${ }^{6}$ University of Defense, Faculty of Military Health Sciences, Hradec Kralove, Czech Republic; ${ }^{7}$ Fundación para el Fomento de la Investigación Sanitaria y Biomédica, Department of Vaccine research, Valencia, Spain; ${ }^{8}$ PrimeHealth Clinical Research, PrimeHealth Clinical Research, Toronto, Canada; ${ }^{9}$ Taipei Veterans General Hospital and National Yang Ming University School of Medicine, Department of Family Medicine, Taipei, Taiwan; ${ }^{10} J a p a n$ Physicians Association, Japan Physicians Association, Tokyo, Japan; ${ }^{11}$ Keyrus Biopharma c/o GSK, Clinical Statistics, Wavre, Belgium; ${ }^{12}$ IWK Health Center and Nova Scotia Health Authority, Canadian Center for Vaccinology, Halifax, Canada; ${ }^{13}$ Uppsala University Hospital, Department of Infectious Diseases, Uppsala, Sweden; ${ }^{14}$ Aichi Medical University, Department of Dermatology, Nagakute, Japan; ${ }^{15}$ Federal University of Sao Paulo, Department of Pediatrics, Sao Paulo, Brazili, ${ }^{16}$ University of Sydney, The Westmead Institute for Medical Research, Sydney, Australia; ${ }^{17}$ GlaxoSmithKline, Clinical Development, King of Prussia, United States, ${ }_{i}^{18}$ GlaxoSmithKline, Clinical Development, Wavre, Belgium; ${ }^{19} \mathrm{GlaxoSmithKline,} \mathrm{Biostatistics,} \mathrm{Wavre,} \mathrm{Belgium;}{ }^{20} \mathrm{GlaxoSmith}-$ Kline, Safety Evaluation \& Risk Management, Wavre, Belgium

Background: The adjuvanted recombinant zoster vaccine (RZV) showed high vaccine efficacy (VE) against herpes zoster $(\mathrm{HZ})$ in adults $\geq 50$ and $\geq 70$ years old ( $Z O E-50$ and -70 trials, respectively). Participants who were immunocompromised or who were receiving immunosuppressive therapy were excluded from the ZOE-50 and -70 trials. Subjects with other medical conditions at baseline, including conditions 
known to be linked to a potential increased risk of $\mathrm{HZ}$, were enrolled in these studies. The study population is a representative sample of the general adult population over the age of 50 years.

Methods: A post-hoc analysis of the pooled ZOE-5O and -70 (ZOE50/70) trials (NCTo1165177; NCTo1165229) assessed VE and safety in adults $\geq 50$ years old reporting at least 1 medical condition at enrollment. The medical conditions included in the analysis were present at enrollment in $\geq 500$ participants in both the placebo and the RZV recipients of the ZOE-5O and -70 . These medical conditions included those that have been recognized to be associated with an increased risk of $\mathrm{HZ}$ (asthma, depression, respiratory disorders, and renal disorders). Fatal/serious adverse events (SAEs) were recorded for at least 12 months after the 2 nd vaccination, potential immune-mediated diseases (pIMDs) were recorded during the entire study period and were assessed with consideration of subjects' baseline medical conditions.

Results: Of the 30,977 participants enrolled in the ZOE-50/70 studies, 13,881 RZV and 14,035 placebo recipients were included in the pooled cohort for this analysis. Of these, $82.3 \%$ of RZV recipients and $82.7 \%$ of placebo recipients had at least 1 of the 15 selected medical conditions at enrollment. A majority (RZV: 59.6\%; Placebo: 59.8\%) reported more than 1 condition. The most frequent baseline medical conditions in the study population were hypertension, osteoarthritis vertebral disorders, and dyslipidemia. VE ranged from $84.5 \%$ (95\% confidence interval [Cl]: 46.4-97.1) (respiratory disorders) to $97.0 \%$ (95\% Cl: $82.3-$ 99.9) (coronary heart disease). Overall, RZV efficacy remained $>90 \%$ irrespective of the number of medical conditions reported by a participant. Frequencies of SAEs, deaths, and pIMDs were balanced between the RZV and the placebo recipients.

Conclusions: Medical conditions were common amongst participants in the ZOE-50/70 trials at enrolment and did not impact RZV vaccine efficacy or safety profile.

Funding: GlaxoSmithKline Biologicals SA

\section{PS101 \\ Utility of MPV, MPV/Platelet Ratio, RDW, RDW/Platelet Ratio, Neutrophil-Lymphocyte Ratio, Platelet-Lymphocyte Ratio and as Diagnostic and Prognostic Markers in Patients with Hepatocellular Carcinoma.}

V.S Srikanth; Pillai M. Gopala Krishna

Amrita Institute of Medical Sciences and Research Centre, Internal Medicine, Kochi, Kerala, India

Background: Liver disease is a growing cause of mortality in India that requires simpler, non-invasive tests for assessing progression and prognosis. We aim to explore the correlation between these indices and severity of HCC as measured by MELD and Child-Pugh scores. We also investigated links between these indices and grades of esophageal varices on Upper GI Endoscopy.

Materials and Methods: This is a retrospective hospital based observational study conducted screening 208,486 patients admitted from January 2013- June 2017 in AIMS, Kochi. Analysed for Neutrophil Lymphocyte Ratio (NLR), Platelet Lymphocyte Ratio (PLR), and EV grading by UGIE.

Results: A total of 196 patients for whom MELD scores, CP Scores, Esophageal variceal grading collected in addition to their admission NLR and PLR values. NLR and PLR showed significant AUROC to MELD Scores with $72.1 \%$ and $65.5 \%$ respectively/ T-Test showed NLR correlating both MELD and Child Scores ( $p-0.053,0.032$ ), while PLR showed insig- nificant ( $p$ - 0.901, 0.095). Mean NLR/PLR for Low MELD scores were 5.12 and 10.36 while those of High MELD were 7.28 and 10.52 respectively. Both indices showed poor AUROC of $59.5 \%$ and $51.6 \%$ in relation to Child-Pugh Score, with Good Child Scores (mean NLR, PLR: 4.03, 8.63) and Poor Child Scores (mean NLR, PLR: 6.62, 11.08).

Conclusion: Our study asserts that NLR may be reliably used to stage and assess progress of background liver disease in patients with HCC in relation to both MELD and Child Scores, while PLR doesn't have the same clinical relevance.

Key Words: HCC, NLR, PLR, Variceal grading

\section{PS102}

Diagnostic Utility of Venous Ammonia Level, Spleen Size and INR as a non-invasive biomarker in predicting oesophageal varices in Liver Cirrhosis

\section{V.S. Srikanth; Henry Renoy}

Amrita Institute of Medical Sciences and Research Centre, Internal Medicine, Kochi, Kerala, India

Introduction: The objective of our study is to evaluate Diagnostic Utility of Venous Ammonia Level, Spleen Size and INR in association to oesophageal Varices in Liver Cirrhosis

Methods: Study setup: Hospital based study. Duration: December 2016 -January 2018 Study population: CLD patients. Variables: serum ammonia level, INR and Spleen size. The test group was divided into 2 groups based on parquet classification of esophageal varices where Groups 1 consisted of (grade I and grade II varices). Group 2 consisted of (grade III and grade IV varices).Inclusion criteria: Patients admitted with a diagnosis of CLD. Exclusion criteria: Patients who receive Endoscopic Variceal Ligation (EVL) or Scelero therapy. Presence of, active GI bleeding. Portal vein thrombosis, Hepatocellular carcinoma

Results: We found a male predominance in the age group of 50-6oyears in CLD patients. Venous ammonia and spleen size were found to be a non-invasive biomarker for predicting oesophageal varices. Where venous ammonia was highly significant ( $p$ value-0.oo) occupying $85 \%$ in ROC analysis. Spleen size was also significant ( $p$-value 0.036) occupying $67 \%$ in ROC analysis. INR was not statically significant

Conclusion: These biomarkers will play a major role predicting oesophageal varices in primary health care setup, which will aid in early diagnosis and prompt referral to higher centre. It will reduce the morbidly and mortality associated with the diseases. Serve as an excellent diagnostic modality in patients whom endoscopy is contraindicated. A non-invasive diagnostic modality for elderly population. These biomarkers will reduce the frequency of repeat endoscopy in CLD patients.

Key Words: Liver Cirrhosis, Oesophageal varices, Spleen Size, Venous Ammonia level. 


\section{PS103}

\section{The Impact of Reactogenicity after Administration of the Recombinant Zoster Vaccine upon the Physical Functioning and Quality of Life of Older Adults}

Johannes Hain'; Kenneth Schmader ${ }^{2}$; Myron Levin ${ }^{3}$; Katrijn Grupping ${ }^{4}$; Sean Matthews ${ }^{11}$; David Butuk ${ }^{5}$; Michael Chen ${ }^{6}$; Mohamed El Idrissi' ${ }^{12}$; Laurence Fissette $^{13}$; Charles Fogarty ${ }^{7}$; Paul Hartley ${ }^{8}$; Nicola Klein ${ }^{9}$; Max Nevares ${ }^{10}$; Kari Uusinarkaus $^{10}$; Lidia Oostvogels ${ }^{14}$; Desmond Curran ${ }^{11}$

${ }^{1}$ GlaxoSmithKline GmbH \& Co. KG, Medical Affairs, München, Germany; ${ }^{2}$ Duke University, Departments of Medicine, Durham, United States; ${ }^{3}$ University of Colorado, Departments of Pediatrics and Medicine, Aurora, United States; ${ }^{4}$ GlaxoSmithKline, Regulatory Affairs, Wavre, Belgium; ${ }^{5}$ Solaris Clinical Research, Solaris Clinical Research, Meridian, United States; ${ }^{6}$ The Corvallis Clinic, Internal Medicine, Corvallis, United States; ${ }^{7}$ Spartanburg Medical Research, Spartanburg Medical Research, Spartanburg, United States; ${ }^{8}$ Preferred Primary Care Physicians, Preferred Primary Care Physicians, Uniontown, United States; ${ }^{9}$ Kaiser Permanente Northern California, Kaiser Permanente Vaccine Study Center, Oakland, United States; ${ }^{10}$ DaVita Medical Group, DaVita Medical Group, Colorado Springs, United States; ${ }^{11}$ GlaxoSmithKline, Health Economics, Wavre, Belgium; ${ }^{12}$ GlaxoSmithKline, Clinical Statistics, Rixensart, Belgium; ${ }^{13} \mathrm{GlaxoSmithKline,} \mathrm{Clinical} \mathrm{Statistics,} \mathrm{Rixens-}$ art, Belize; ${ }^{14}$ GlaxoSmithKline, Clinical and Epidemiology, Wavre, Belgium

Background: Herpes zoster $(\mathrm{HZ})$ and its related complications are associated with a significant burden of illness in older adults, which negatively impacts patients' physical functioning and quality-of-life (QoL). The recombinant zoster vaccine (RZV) shows high efficacy for the prevention of $\mathrm{HZ}$ in older adults and is associated with local and systemic reactions. Therefore, this study assessed the impact of RZV reactogenicity upon the physical functioning and $Q$ oL of participants.

Methods: 401 adults aged $\geq 50$ years received a dose of RZV at o and 2 months in this open-label, single-arm, multicenter study (NCT02979639). Changes in mean SF-36 Physical Functioning score were assessed between pre-dose- 1 vaccination and post-dose- 1 vaccination for 7 days (primary endpoint). Decreased scores are associated with decreased physical functioning. QoL, reactogenicity and safety were also assessed. The current analysis was performed post-dose-1 vaccination of the 2-dose RZV schedule.

Results: No clinically meaningful reductions in overall mean SF-36 Physical Functioning scores from pre- to post-RZV dose-1 were observed (mean +1.9 points) and no overall quality-adjusted-life-year loss was recorded post-dose-1. However, grade 3 reactogenicity occurred in $9.5 \%$ of participants, and was associated with a transient, clinically-important decrease in SF-36 Physical Functioning score (impacting activities such as walking, carrying groceries, climbing stairs) on Days 1-2 post-first-vaccination. The solicited local symptoms were pain $(77.5 \%)$, redness $(23.0 \%)$ and swelling $(13.3 \%)$; the most frequent solicited systemic reactions were fatigue $(33.5 \%)$, headache $(28.3 \%)$ and myalgia (26.8\%).

Conclusion: Overall, the physical functioning and QoL of older adults were not significantly affected by the first RZV dose. Grade 3 reactogenicity was associated with a small transient decrease in physical functioning 1-2 days post-dose-1 that resolved by Day 3 post-vaccination.

Funding: GlaxoSmithKline Biologicals SA
PS104

SEAL-Programm - Strukturierte Früherkennung von Leberfibrose und Leberzirrhose durch Screening der Allgemeinbevölkerung

Michael Nagel'; Anita Arslanow'; Marc Nguyen-Tat ${ }^{2}$; Marcus A. Wörns'; Franz Josef Heil ${ }^{3}$; Dagmar Mainz ${ }^{4}$; Gundula Zimper ${ }^{5}$; Burkhard Zwerenz ${ }^{6}$; Julian Wangler ${ }^{10}$; Michael Jansky ${ }^{10}$; Johannes Jäger ${ }^{7}$; Desiree Gisch ${ }^{7}$; Tobias Engelmann ${ }^{11}$; Erika Graf ${ }^{8}$; Jochen Knaus ${ }^{8}$; Dominikus Stelzer ${ }^{8}$; Urs A. Fichtner $^{8}$; Harald Binder ${ }^{8}$; Erik Farin-Glattacker ${ }^{8}$; Julia Ortner ${ }^{9}$; Reyn van Ewijk'; Louis Velthuis ${ }^{12}$; Frank Lammer ${ }^{13}$; Peter R. Galle ${ }^{1}$

'Universitätsmedizin Mainz, I. Medizinische Klinik und Poliklinik, Mainz, Germany; ${ }^{2}$ Klinikum Osnabrück, II. Medizinische Klinik, Osnabrück, Germany; ${ }^{3}$ Berufsverband Niedergelassener Gastroenterolgen (bng), Berufsverband Niedergelassener Gastroenterolgen (bng), Andernach, Germany; ${ }^{4}$ Berufsverband Niedergelassener Gastroenterologen (bng), Berufsverband Niedergelassener Gastroenterologen (bng), Andernach, Germany; ${ }^{5}$ Saarländischer Hausärzteverband, Saarländischer Hausärzteverband, Kirkel, Germany; ${ }^{6}$ Deutscher Hausärzteverband-Landesverband Rheinland-Pfalz, Deutscher Hausärzteverband-Landesverband Rheinland-Pfalz, Koblenz, Germany; ${ }^{7}$ Universitätsklinikum des Saarlandes, Zentrum für Allgemeinmedizin, Homburg, Germany; ${ }^{8}$ Universitätsklinikum Freiburg, Institut für Medizinische Biometrie und Statistik, Freiburg, Germany; ${ }^{9}$ Johannes Gutenberg-Universität Mainz, Lehrstuhl für Statistik und Ökonometrie, Mainz, Germany:10Universitätsmedizin Mainz, Zentrum für Allgemeinmedizin und Geriatrie, Mainz, Germany; ${ }^{11}$ Universitätsmedizin Mainz, Interdisziplinäres Zentrum Klinische Studien (IZKS), Mainz, Germany; ${ }^{9}$ Johannes Gutenberg-Universität Mainz, Lehrstuhl für Statistik und Ökonometrie, Mainz, Germany; ${ }^{12}$ Johannes Gutenberg-Universität Mainz, Lehrstuhl für Controlling, Mainz, Germany; ${ }^{13}$ Universitätsklinikum des Saarlandes, Klinik für Innere Medizin II - Gastroenterologie und Endokrinologie, Homburg, Germany;

Einleitung: Die Diagnose Leberzirrhose wird selbst in Ländern mit hoch entwickeltem Gesundheitssystem wie Deutschland bei $75 \%$ der Patienten erst mit dem Auftreten von Komplikationen gestellt. Patienten mit dekompensierter Leberzirrhose haben ein hohes Risiko für die Entwicklung weiterer Folgeerkrankungen. Dabei sind die meisten chronischen Lebererkrankungen, die zu Leberfibrose führen, einer ursächlichen Behandlungsintervention zugänglich.

Ziele: Bisher ist in der Regelversorgung keine Leber-Früherkennung etabliert. Das SEAL-Projekt (Strukturierte Früh-Erkennung einer Asymptomatischen Leberzirrhose in Rheinland-Pfalz und im Saarland) untersucht die Umsetzbarkeit, Effektivität und Kosten-Nutzen-Bewertung eines allgemeinen Screenings auf Leberfibrose.

Patienten und Methodik: Im Rahmen des SEAL-Projekts wird in Rheinland-Pfalz und im Saarland bei ca. 16.000 Versicherten der AOK im Rahmen des hausärztlichen Check-Up-35 ein Screening auf Alanin-Aminotransferase (ALT)-Erhöhung durchgeführt. Bei Überschreiten eines Leberfibrose-Risikoscore erfolgt die Überweisung an einen Gastroenterologen zur weiteren differenzialdiagnostischen Abklärung. Erhärtet sich der Verdacht auf eine Leberfibrose, erfolgt die Vorstellung in einem universitären Leberzentrum zur Diagnostik und Therapie. Die Abklärung wird durch eine transsektorale Web-Plattform unterstützt, die allen Behandlern den Zugriff auf Ergebnisse der Diagnostik ermöglicht. Endpunkte sind neben der Leberfibrose-Diagnoserate Daten zur Epidemiologie von Leberwerterhöhungen sowie zur Kosten-Nutzen-Bewertung.

Umsetzung: Das SEAL-Projekt wird vom G-BA-Innovationsfonds gefördert und von einem Konsortium aus Universitätsmedizin Mainz, Universität des Saarlandes, den Hausärzteverbänden in Rheinland-Pfalz und im Saarland, dem Berufsverband Niedergelassener Gastroenterologen (bng) sowie der AOK Rheinland-Pfalz/Saarland umgesetzt. Die Evaluation erfolgt durch die Universitäten Freiburg 
und Mainz. Die Rekrutierung von Haus- und Fachärzten begann im November 2017, der erste Patienten wurde April 2018 eingeschlossen. Aktuell nehmen im Dezember 2018 fast 300 Ärzte teil, die bisher über 1000 Patienten rekrutiert haben.

Schlussfolgerungen: Die Etablierung einer Früherkennung von Lebererkrankungen wird von Fachgesellschaften und in der Literatur kontrovers diskutiert. Das SEAL-Projekt wird Daten zu Umsetzbarkeit, Effektivität und Kosten-Nutzen-Bewertung im deutschen Gesundheitswesen liefern und wesentliche Evidenz zur Sinnhaftigkeit einer solchen Maßnahme beitragen.

\section{Seltene Erkrankungen}

\section{PS105 \\ Fieber, Kopfschmerzen u. Sturzsenkung bei einer 70 jährigen Patientin - Die Krönung ist die Lösung.}

\section{Jens Bester; Peter Härle}

Katholisches Klinikum Mainz, Klinik für Rheumatologie, Klinische Immunologie u. Physikalische Therapie, Mainz, Germany

Fallpräsentation: Eine 70-jährige Rentnerin stellte sich im September 2018 in unserer ZNA vor. $\mathbf{2}$ Wochen vor Aufnahme klagte sie über linksseitige Knieschmerzen, die unter NSAR Gabe wieder verschwanden. Eine Woche später bekam sie intensivste Nackenkopfschmerzen mit haubenförmiger Ausstrahlung und Fieber bis $39{ }^{\circ} \mathrm{C}$. Ein ambulantes MRT HWS hatte lediglich einen Bandscheibenvorfall HWK 6/7 ergeben. Bei stark erhöhten Entzündungswerten erfolgte die Einweisung in unsere Klinik. Diagnostik: In der klinischen Untersuchung zeigte sich die Patientin in reduziertem AZ mit führend schmerzbedingter HWS Bewegungseinschränkung insbesondere ohne Meningismus und ohne periphere Arthritis. Im Labor sahen wir eine ausgeprägte Entzündungsreaktion bestehend aus Leukozytose, Sturzsenkung und CRP-Erhöhung. Röntgen Thorax, U-Stix, Sono Abdomen und Echokardiographie waren noch in der ZNA ohne wegweisenden Entzündungsfokus, so dass die Aufnahme in die Klinik für Rheumatologie erfolgte. Differentialdiagnostisch kam entsprechend den EULAR-ACR-Klassifikationskriterien aufgrund des Alters, der akuten Kopfschmerzsymptomatik und der hohen Entzündungswerte eine Riesenzellarteriitis in Betracht. Die duplexsonographische Gefäßevaluation war jedoch diesbezüglich unauffällig. Die genaue Anamnese einer vor Beginn der Kopfschmerzsymptomatik stattgehabten akuten Gonarthritis führte trotz momentaner Beschwerdefreiheit an den Knien zur Arthrosonographie und damit Detektion einer für eine Chondrocalcinose typischen Meniskusverkalkung. Therapie und Verlauf: Unter dem klinischen Verdacht eines cervicalen Pseudogichtanfalls einer Calcium-Pyrophosphat-Erkrankung (CPPD) erfolgte eine Therapie mit einem Coxib und Colchicin, worunter die Patientin innerhalb von $48 \mathrm{Std}$. beschwerdefrei war. Das ergänzende CT HWS bestätigte die Verdachtsdiagnose einer atlantodentalen Chondrocalcinose im Sinne eines "Crowned-Dens-Syndrome". Zusammenfassung und Diskussion: Akute Cervicocephalgien stellen einen häufigen Vorstellungsgrund sowohl ambulant wie auch im Notaufnahme Setting dar. Da häufig keine weiteren Warnsymptome wie Fieber vorliegen, wird meist eine symptomatische Therapie ohne weitere Diagnostik verordnet. Trotz fehlender radikulärer Symptomatik erfolgt zunehmend auch aus Strahlenschutzgründen ein MRT mit der Frage einer discogenen Ursache. Das Crowned-Dens-Syndrome kann wie in unserem Fall hierbei leicht übersehen werden, da die Chondrocalcinose röntgenologisch besser zur Darstellung kommt und der atlantodentale Übergang je nach Fragestellung im MRT nicht im Fokus steht. Aus diesen Gründen ist die Wirbelsäulen-Beteiligung und insbesondere die cervicale CPPD eine wenig bekannte und sicher unterdiagnostizierte Manifestation der seit 1962 von McCarty erstmals beschriebenen und im klinisch-rheumatologischen Alltag häufigen Kristallarthritis. In der Abgrenzung zur Riesenzellarteriitis stellt die cervicale CPPD eine wichtige Differentialdiagose dar.

\section{PS106 \\ Adenosine signaling does not skew $T$ helper cell differentiation in vitro but might be involved in persistent $\mathrm{T}$ cell activation in ANCA-associated vasculitis}

Lisa Leikeim; Bernhard K. Krämer; Benito A. Yard; Anna-Isabelle Kälsch

Universitätsmedizin Mannheim, V. Medizinische Klinik, Mannheim, Germany

A recent prospective study has demonstrated expansion of effector Th17 cells in ANCA-positive granulomatosis with polyangiitis (GPA) patients and showed that increased Treg and Th2 lymphocytes is a characteristic feature of sustained remission in such patients (Szczeklik, Jakiela et al. 2017). In keeping with the emerging role of adenosine to shape immune responses and our recent finding that in lymphocytes of ANCA associated vasculitis (AAV) patients the expression of enzymes regulating extra-cellular adenosine (CD73, CD39 and $C_{2}$ 26) is changed (Kling, Benck et al. 2017), we assessed to what extent concurrent adenosine signaling influences Th differentiation at the time of T-cell activation.

To this end, gene expression profiling, FACS analysis and cytokine production measurement was performed on anti-CD3/anti-CD28 activated $\mathrm{CD}_{3}+\mathrm{T}$ cells of healthy donors $(\mathrm{N}=3)$ cultured in the presence or absence of an unspecific adenosine receptor agonist 5 '-(N-Ethylcarboxamido)adenosine (NECA).

While T-cell proliferation and IL-2 production were significantly down-regulated by NECA, IL-10 and IL-17A were increased. Inhibition of proliferation coincided with down-regulation of genes belonging to cell cycle progression. In Kegg pathway analysis, negatively enriched pathways mainly clustered in the categories metabolism and genetic information processing while positively enriched pathways mainly clustered in signal transduction and immune system. Analysis of master transcription factors for Th differentiation, i.e. TBET, GATA3, RORyt and FOXP 3 as well as members of the STAT family and markers for Th9, Th22 and Tfh differentiation revealed no consistent up- or down-regulation in the presence of NECA. Changes in the adenosinergic system were confined to up-regulation of $C_{2} 26$ in the presence of NECA, which was confirmed by FACS.

Since concurrent adenosine signaling at the time of T-cell activation does not find its reflection in Th lineage commitment but rather in increased IL-17 production, changes in CD73, CD 39 and CD26 in T-cells of AAV patients are unlikely directly involved in expansion of Th17 and impairment of Treg as a consequence of decreased extracellular adenosine concentrations. However, because adenosine profoundly inhibits T-cell activation, a relative shortage of extracellular adenosine may explain persistent T-cell activation in AAV patients.

Kling, L., U. Benck, A. Breedijk, L. Leikeim, M. Heitzmann, S. Porubsky, B. K. Kramer, B. A. Yard and A. I. Kalsch (2017). "Changes in CD73, CD39 and $\mathrm{CD}_{26} 6$ expression on T-lymphocytes of ANCA-associated vasculitis patients suggest impairment in adenosine generation and turn-over." Sci $\operatorname{Rep} 7(1): 11683$.

Szczeklik, W., B. Jakiela, K. Wawrzycka-Adamczyk, M. Sanak, M. Hubalewska-Mazgaj, A. Padjas, M. Surmiak, K. Szczeklik, J. Sznajd and J. Musial (2017). "Skewing toward Treg and Th2 responses is a characteristic feature of sustained remission in ANCA-positive granulomatosis with polyangiitis." Eur J Immunol 47(4): 724-733. 


\section{PS107}

\section{Association of somatic mutations in PPM1D and TP53 in myelodysplastic syndromes with complex cytogenetic aberrations}

Lea Eder; Christina Ganster; Katayoon Shirneshan; Katharina Rittscher; Roxana Schaab; Roman Martin; Sascha Dierks; Detlef Haase

Universitätsmedizin Göttingen, Klinik für Hämatologie und medizinische Onkologie, Göttingen, Germany

Introduction: Myelodysplastic syndromes (MDS) are a heterogeneous group of rare clonal hematopoietic stem cell diseases that lead to different types of cytopenia. The incidence in Germany is 5/100.000 inhabitants per year. Cytogenetics is of high relevance for prognostication, classification and personalized allocation of therapy in MDS. Complex aberrations ( $\geq 3$ cytogenetic aberrations, $c A$ ) to date represent one of the most unfavorable prognostic markers. About $50 \%$ of patients (pts) with cA show TP53 mutations that "allow" cell clones with CA to survive and expand. They might further worsen the prognosis of pts with CA. The gene PPM1D located on chromosome 17923 encodes the protein Wip1. It acts as an inhibitor of p53. About $5 \%$ of MDS pts with $5 q$ deletion show mutations in PPM1D (Panagiota et al., ASH 2017) and they are even more common in pts with therapy-related MDS (tMDS, 15\%, Lindsley et al., 2017). The aim of our study was to determine the frequency of PPM1D mutations in MDS pts with cA with and without TP53-mutations and their influence on survival.

Methods: Pts were characterized by conventional cytogenetics and fluorescence in situ hybridization (FISH) containing a FISH-probe covering the TP53 locus. By now 78 pts (51x MDS, 24 x sAML $3 \times$ CMML) with known TP53 status have been identified and were subjected to next generation sequencing (NGS) of PPM1D. Our cohort comprised 70 pts with CA (median age: $71 \mathrm{yrs}$, range 25-95; median number of cytogenetic aberrations: 7, range 3-50; 14 pts with tMDS) and 8 tMDS without CA (median age: 73 yrs, range: 65-79).

Results: A biallelic mutation of TP 53 (mutation and deletion) was present in 25 pts (32\%), further 19 (24\%) had aTP53 mutation without deletion. A deletion without mutation was present in three pts (4\%). All pts without $C A$ were TP53 wildtype. We included 22 pts with tMDS, 14 of them with cA.

In five pts we found a sequence variant of PPM1D. All five pts had CA. Two variants have already been described as very rare SNPs. The other three have not been described before. Two of them are at the same position in exon 1, the third very close to the others. All of them are potential missense mutations. One of those three pts shows a TP53 mutation. None of them showed a deletion in $5 q$. The median number of aberrations in these three pts was 4 (range: $4-8$ ).

Conclusion: As an inhibitor of TP53, and potential pathogenetic alternative to mutations of TP53 itself, we hypothesized that the gene encoding for Wip1, PPM1D, might be mutated in MDS pts with cA but without TP53 mutation, especially in tMDS. We could show that PP$M_{1} D$ is mutated in pts with cA. In our cohort $7 \%$ of the pts with cA are affected. One of them had a tMDS. PPM1D mutations might contribute to the pathogenesis of $\mathrm{CA}$ as $2 / 3$ potential mutations do not $\mathrm{CO}-\mathrm{OC}-$ cur with a TP53 mutation. To further determine the influence on the prognosis we will test the PPM1D mutation status in a higher number of MDS pts. Respective analyses are on the way.
PS108

\section{Das Aneurysma der A. carotis: selten aber nicht ungefährlich}

Spiridon Botsios; Erik Biegel; Omar Khader; Joachim Trunk

Klinikum Lüdenscheid, klinik für Gefäßchirurgie, Lüdenscheid, Germany

Einleitung: Das Aneurysma der extrakraniellen A. carotis ist sehr selten, sollte aber aufgrund der Entwicklung von möglichen Komplikationen einer Behandlung zugeführt werden. Das optimale Behandlungsmanagement steht unter Diskussion. Wir möchten anhand dreier Fallberichte über die chirurgische Behandlung von extrakraniellen Aneurysmen der A. carotis berichten.

Fallbericht I: Eine 73-jährige Patientin hatte eine zunehmende Schwellung am linken Halsbereich bemerkt. Im Rahmen der weiterführenden Diagnostik wurde duplexsonographisch ein Aneurysma der linken A. carotis interna (ACl) festgestellt. Das Aneurysma konnte komplett abgetragen werden und die Kontinuität der $\mathrm{ACl}$ wurde durch End-zu-End-Anastomose spannungsfrei wiederhergestellt.

Fallbericht II: Ein 48-jähriger Patient wurde aufgrund von transitorischen, ischämischen Attacken vorstellig. In der Duplexsonographie fand sich ein Aneurysma der linken ACl als Ursache der Beschwerden. Es wurde die Indikation zur dringlichen Operation gestellt. Das Aneurysma wurde in toto abgetragen. Die Absetzungsstelle wurde über Fortführung der Inzision in die A. carotis externa hinein nach proximal verlängert und mit einem Pericardpatch verschlossen. Abschließend wurde die $\mathrm{ACl}$ an der Perikardpatchplastik durch End-zu-Seit-Anastomose reimplantiert.

Fallbericht III: Ein 82-jähriger Patient berichtete über eine zunehmende Schwellung an der rechten Halsseite. Die Duplexsonographie zeigte ein Aneurysma der A. carotis communis und einen Verschluss $\operatorname{der} \mathrm{ACl}$ rechts. Das Aneurysma wurde operativ komplett abgetragen und ein carotido-carotideales Veneninterponat angelegt.

Schlussfolgerung: Die operative Rekonstruktion stellt eine sichere Behandlungsoption für Aneurysmen der extrakraniellen A. carotis dar. Aufgrund von anatomischen Einschränkungen ist aber die Rekonstruktion nicht immer möglich. In solchen Fällen bietet die Bypasschirurgie eine weitere operative Behandlungsoption. Bei ausgewählten Fällen kann ebenso die endovaskuläre Behandlung eine Alternative zu der Operation sein.

\section{PS109 Hyper-IgE-Syndrom als Differentialdiagnose bei Zystischem Lungenbefall}

Andreas Bucher'; Stefan Zielen²; Benjamin Siebald²; Johannes Schulze ${ }^{2}$; Martin Beeres'; Thomas Vogl'

'Universitätsklinikum Frankfurt am Main, Zentrum der Radiologie, Institut für Diagnostische und Interventionelle Radiologie, Frankfurt am Main, Germany; ${ }^{2}$ Universitätsklinikum Frankfurt am Main, Allergologie, Pneumologie und Mukoviszidose, Klinik für Kinder- und Jugendmedizin,, Frankfurt am Main, Germany

Hintergrund: Das Hyper-IGE Syndrom (HIES) stellt einen genetischen Defekt mit autosomal rezessiven und autosomal dominanten Varianten dar. Der Langzeitverlauf von HIES-Patienten ist nur spärlich in der Literatur beschrieben. Typische Gründe der Hospitalisation beinhalten Pneumonie, Hautinfekte, und Sepsis. Die Sepsis wird als häufigster Todesgrund angegeben (1). Ein Lungenbefall bei HIES-Patienten ist CT-morphologisch gut zu diagnostizieren, und stellt eine seltene Entität dar. 
Material \& Methodik: Es erfolgt die Darstellung von zwei HIES-Patientinnen mit zystischem Lungenbefall.

\section{Ergebnis:}

Fall 1: 15 Jahre, weiblich; Die CT-Thorax-Untersuchung zeigt ausgeprägte zystisch-destruktive Veränderungen der Lunge beidseits, links-betont. Die Patientin ist in domo seit 7 Jahren in Behandlung. Staphylokokken-assoziierte Abszesse inklusive Mundbereich im ersten Lebensmonat vorbeschrieben, im Verlauf Jahren kam es, ab dem 4. Lebensjahr, zu einer zunehmenden pulmonalen Symptomatik mit rezidivierenden Staphylokokken-Pneumonien. Die Therapie mit Rituximab und Immunglobulinen sowie Azithromycin-Prophylaxe zeigt eine deutlich bessere Belastbarkeit und Verbesserung der Lebensqualität.

Fall 2: 4 Jahre, weiblich; Vorstellung mit unklarer cystischen Lungenerkrankung. Die aktuelle CT zeigt den Zustand nach Pneumektomie rechts sowie multiple Zysten nun auch computertomographisch gesichert in der linken Lunge. Die radiologische Erstbeschreibung des zystischen Lungenbefalls erfolgte im zweiten Lebensjahr. Angegeben werden zudem Ekzeme im Säuglingsalter, rezidivierende Pneumonien, eine Abszedierung nach TBC-Impfung, eine pulmonale Abszedierung und eine rechts skoliotische Fehlhaltung. Es wurde ebenfalls eine Azithromycin-Prophylaxe eingeleitet.

Schlussfolgerung: Bei der radiologischen Beschreibung eines zystischen Lungenbefalls bei pädiatrischen Patienten sollte differentialdiagnostisch das Hyper-IgE-Syndrom in Erwägung gezogen werden. Nur so kann eine Verbesserung der Lebensqualität neben einer kausalen Therapie (i.e. Knochenmarkstransplantation, Rituximab-Therapie) adäquat angeleitet werden.

[1] S. Alyasin, H. Esmaeilzadeh, N. Ebrahimi, S.H. Nabavizadeh, S. Kashef, E. Esmaeilzadeh, M. Babaei, R. Amin, Phenotyping and long-term follow up of patients with hyper IgE syndrome, Allergologia et immunopathologia (2018).

\section{PS110 \\ Sichelzellenanämie einer älteren Patientin mit multiplen Komplikationen - ein Fallbericht}

Christian Benignus; Veronica Belling; Serkan Karakaya

Marienhospital Stuttgart, Innere Medizin ${ }^{3}$, Stuttgart, Germany

Eine Punktmutation an der 6 . Stelle des $\beta$-Globin-Gens tauscht die Aminosäure Glutaminsäure durch Valin aus, sodass sich die betroffenen Erythrozyten nach O2-Abgabe sichelförmig verformen. Aufgrund des autosomal kodominanten Erbgangs erkranken nur die homozygot Betroffenen. Heterozygote Überträger werden erst unter schwerstem O2-Mangel symptomatisch. Die Sichelzellenanämie bietet außerdem einen erheblichen Schutz gegenüber der Malaria tropica (Infektion mit Plasmodium falciparum) und stellt somit einen Selektionsvorteil - vor allem in Subsahara Afrika - dar. Die Sichelzellenanämie des Erwachsenen stellt in Deutschland bisher eine Rarität für den Internisten dar, wird aber - auch aufgrund der Migrationsbewegungen - in Zukunft häufiger zu finden sein. Obwohl Patienten mit einer Sichelzellenanämie heutzutage deutlich länger überleben, erreichen sie im Median gerade einmal eine Lebenserwartung von ca. 60 Jahren.

Wir berichten von einer 66-jährigen Patientin, die in reduziertem Allgemeinzustand und mit generalisiertem Schmerz (vor allem der Hüften) sowie Atemnot vorstellig wurde. Bei Aufnahme zeigten sich folgende Laborwerte: $\mathrm{Hb}: 4,6 \mathrm{~g} / \mathrm{dl}, \mathrm{K}+: 5,5 \mathrm{mmol} / \mathrm{l}$; Ca2+: 2,9 mmol/l, LDH: 1186 U/l, Gesamt-Bilirubin: 4,8 mg/dl. Es wurde die Diagnose einer hämolytischen Anämie sowie eines akuten Nierenversagens mit Hyperkaliämie und Hypercalcämie sowie renaler, metabolischer Azidose gestellt. Zusätzlich verursachte die Nephropathie eine arterielle Hypertonie mit diastolischer Dysfunktion und hypertensiver Herzkrankheit sowie pulmonaler Hypertonie. Die letzte Bluttransfusion aufgrund der Erkrankung erhielt die Patientin 28 Jahre vor der Einlieferung in unsere Klinik. Seitdem hat keine weitere Behandlung stattgefunden.

Therapeutisch bekam die Patientin zum Ausgleich der Azidose Natriumhydrogencarbonat i.v. und später peroral, außerdem Kaliumbinder zur Behandlung der Hyperkaliämie und drei Erythozytenkonzentrate aufgrund der Anämie. Die schwere Hypercalcämie verbesserte sich deutlich nach einer forcierten Diurese und nach der Gabe von Denosumab. Zudem wurde die Hypertonie mit ACE-Hemmern eingestellt und es wurde eine Schmerztherapie nach WHO-Stufe 2 begonnen. Versuchsweise wurde außerdem eine regelmäßige EPO-Substitution eingeleitet, unter der es bis zum jetzigen Zeitpunkt zu keinen weiteren hämolytischen Krisen mehr gekommen ist, deren längerfristiger Nutzen jedoch noch abgewartet werden muss. Die Patientin konnte so nach wenigen Wochen mit annähernd normwertigen Laborwerten entlassen werden.

\section{PS111}

\section{Akzidentelle Spätmanifestation einer Zystischen Fibrose bei rezidivierenden Pankreatitiden}

Patricia-luliana Mester'; Lukas Moleda; Martina Müller-Schilling²

'Universitätsklinikum Regensburg, Innere Medizin I, Regensburg, Germany; 2Universitätsklinikum Regensburg, Klinik und Poliklinik für Innere Medizin I, Regensburg, Germany

Hintergrund: Zystische Fibrose gehört zu den häufigsten, genetisch prädeterminierten lebensverkürzenden Erkrankungen. Pathophysiologisch liegt eine Mutation im Cystic Fibrosis Transmembrane Conductance Regulator Gen (CTFR) mit konsekutiver Störung der Chloridkanäle exokriner Drüsen vor. Bereits im Kindesalter können sich auch extrapulmonale Manifestationen zeigen. Die Pankreatitis ist hierbei mit ca. 1-2 \% der Fällen vertreten. Spätmanifestationen sind hingegen deutlich seltener anzutreffen. Gesichert ist, dass heterozygote CFTR-Mutationsträger zwei bis elfmal anfälliger für eine idiopathische chronische Pankreatitis sind.

Klinischer Fall: Wir berichten über den Fall eines gesunden männlichen 35-jährigen Patienten mit rezidivierenden, initial unklaren Pankreatitiden, die erstmalig im Jahre 2015 aufgetreten sind. Bis einschließlich 2018 erlitt der Patient insgesamt sechs weitere Schübe einer akuten Pankreatitis, mit letztendlich unklarer Ätiologie. Laborchemisch imponierte in der Akutphase der Pankreatitis klassisch eine kräftige Lipaseerhöhung. Zudem beklagte der Patient die typische Schmerzsymptomatik.

Im Rahmen der Ursachensuche erfolgte eine endosonographische Punktion mit histologischer Sicherung, welche ebenfalls nicht richtungsweisend blieb. Ferner erbrachte auch die weiterführende Diagnostik keinen eindeutigen Hinweis auf die Ursache. Insbesondere konnten die klassischen Ursachen einer Pankreatitis, z.B. biliär, IgG4-assoziiert, nutritiv-toxisch, traumatisch oder medikamentös ausgeschlossen werden. Erst im Rahmen der hereditären Abklärung konnte eine heterozygote Mutation im CFTR-Gen nachgewiesen werden. Die hierzu komplementär durchgeführte Lungenschnittbildgebung zeigte typische pulmonale Veränderungen einer Zystischen Fibrose. Eine passende pulmonale Symptomatik manifestierte sich nie. Eine zusätzlich durchgeführte Konfirmationsdiagnostik bestätigte mittels Schweißtest die Verdachtsdiagnose einer Zystischen Fibrose. 
Diskussion und Schlussfolgerung: Die hereditäre Pankreatitis ist eine seltene Erkrankung. Diese ist mit Mutationen der PRSS1-, SPINK1und CFTR-Gene assoziiert. Aktuelle genetische Untersuchungen zeigen zudem, dass die chronisch-idiopatische Pankreatitis häufig mit zusätzlichen, gleichzeitig vorkommenden Mutationen assoziiert ist.

Unser Fall ist dahingehend von großem Interesse, da die zugrundeliegende bis dato inapparente Zystische Fibrose als Zufallsbefund bei einem Patienten mit rezidivierenden Pankreatitiden diagnostiziert wurde. Diesbezüglich kann nun die entsprechende Vorsorge getroffen werden. Dieser Fall unterstreicht die Bedeutung einer genetischen Untersuchung im Rahmen unklarer Erkrankungsbilder junger Patienten.

\section{PS112}

\section{7-Year Old Tunisian Male with reccurent Acute Scrotum and Fever}

Rim Dhaouadi

\section{BIZERTE, Tunisia}

Case report: A 17-Year old Tunisian male, presented to the emergency with a right painful swollen scrotum and high fever $\left(39^{\circ} \mathrm{C}\right)$ starting the day before the admission.No context of trauma was reported. Physical examination revealed a swollen erythematous right scrotum. Cremastic reflexes were present. He had no abdominal pain or other symptoms. Lab results showed leucocytosis, an elevated erythrocyte sedimentation rate and a mildly elevated C-reactive protein level. Cytobacteriological examination of urine was negative. Scrotal and abdominal ultrasonography showed epididymal and testicular enlargement and a thickened scrotal skin.Thus, testicular torsion was excluded and the diagnosis of unilateral epididymo-orchitis was considered. Recovery was spontaneous within four days. Five weeks later, the patient was admitted in internal medecine departement for similar symptoms enluding epigastric pain with no other digestive disorder. Lab results were normal except for a significantly elevated erythrocyte sedimentation rate. Abdominal-CT and upper endoscopy were normal. Specific serological tests for CMV, EBV, HIV, Hepatitis $B$ and $C$ as well as Widal and Wright serologies (patient originated from an endemic area) were performed and came back all negative. PPD skin test was negative too. Autoimmune anti-bodies (ANA) were not detectable. Additional history revealed that the patient had during the last eight months four similar attacks of abdominal pain with fever lasting no longer than two or three days, resolving spontaneously. Because of his mediterranean origin, Familial Mediterranean Fever was suspected. Colchicine therapy was initiated. A followup for 12 months showed that he had no similar episodes.

Discussion: Familial Mediterranean Fever (FMF) is a genetic disease caracterized by idiopathic, self-limited attacks of fever mostly associated with inflammation of serosae. The most commun presenting symptoms enclude fever, abdominal or chest pain, arthiritis, myalgia and erysipelas-like erythema. Acute scrotum was the presenting feature of FMF in rare reported cases in literature $(1,2)$. With our patient, the first two episodes were not characteristic of the diagnosis, however, short attacks of fever, swelling, association with new symptoms and recurrence in a subject of Mediterranean origin (especially that other differential diagnosis of acute scrotum were ruled out by physical examination, laboratory and radiological test findings), strongly points toward the diagnosis of FMF. In that case, Colchicine was given with the purpose of controlling the disease and preventing its most fatal complication which is Amyloidosis(3).

Conclusion: Acute scrotum is a rare manifestation of FMF, but, with the absence of objective markers and the recognition of the symp- toms in individuals with Mediterranean origins, this disease should be considered and treated.

[1] Yilmaz R, Ozer S. A rare presentation of familial mediterranean Fever; acute scrotum and hydrocele amyloidosis. Iran J Pediatr. sept 2010;20(3):367-9.

[2] Majeed HA, Ghandour K, Shahin HM. The acute scrotum in Arab children with familial Mediterranean fever. Pediatr Surg Int. 2000;16(1-2):72-4.

[3] Colchicine for Familial Mediterranean Fever. N Engl J Med. 12 avr 1973;288(15):798-798.

\section{PS113}

Novel insights into postprandial hypoglycemia after gastric bypass: Empagliflozin as well as Anakinra reduce symptomatic episodes by lowered insulin secretion

Matthias Hepprich;; Benjamin Schelker'; Alessandra Staerkle ${ }^{2}$; Beckey Trinh'; Sophia Wiedemann'; Marianne Böni-Schnetzler'; Gottfried Rudofs$\mathrm{ky}^{3}$; Marc Donath

'Universitätsspital Basel, Klinik für Endokrinologie, Diabetologie und Metabolismus, Basel, Switzerland; ${ }^{2}$ Swiss Federal Institute of Technology Zurich, Zurich, Switzerland, Department of Health Sciences and Technology , Zürich, Switzerland; ${ }^{3}$ Kantonsspital Olten, Stoffwechselzentrum, Olten, Switzerland; 'Universitätsspital Basel, Klinik für Endokrinologie, Diabetologie und Metabolismus, Basel, Switzerland

Background: Postprandial hypoglycemia after bariatric surgery is characterized by a pronounced glycemic rise after carbohydrate ingestion and an exaggerated hyperinsulinemic response(1). Recent studies have shown that IL-1 $\beta$ contributes to the postprandial stimulation of insulin(1), and that bariatric surgery affects the gut flora and associated inflammatory response. Furthermore, inhibition of the SGLT2 may reduce excessive plasma glucose increase. Therefore, we investigated whether inhibition of IL-1 $\beta$ with the IL-1 receptor antagonist anakinra and/or inhibition of SGLT2 with empagliflozin reduces postprandial hypoglycemia after bariatric surgery.

Methods: In this placebo controlled, double-blind, randomized, cross-over proof-of-concept study 12 subjects with confirmed postprandial hypoglycemia after gastric bypass were included. Subjects received on each of the 3 study days either empagliflozin p. o. or anakinra s. c. along with the respective placebo or double placebos. Three hours after injection (anakinra or placebo) and two hours after ingestion of the oral study medication (empagliflozin or placebo) a mixed-meal-test was performed with assessment of hypoglycemia. Clinical assessments (Edinburgh hypoglycemia scale, mini mental status test, Sigstad score, Stanford sleepiness Scale) were performed aside measurement for glucose, insulin, c-peptide as well as inflammatory parameters.

Results: Empagliflozin reduced peak glycaemia at 30 (11.2 vs. 10.1 $\mathrm{mmol} / \mathrm{l}), 60$ (9.1 vs. $6.9 \mathrm{mmol} / \mathrm{l})$ and 90 (4.5 vs. $3.5 \mathrm{mmol} / \mathrm{l})$ minutes after ingestion of the mixed meal compared to placebo and was followed by a significant reduction of glucose-requiring hypoglycemic events ( $n=2,16.6 \%$ ) compared to placebo $(n=8,61.5 \%)$. In contrast, treatment with Anakinra did not result in significant changes of the glucose curve within the first 90 minutes, but was also followed by a significantly reduced rate of glucose-requiring hypoglycemic events $(n=2,16.6 \%)$ compared to placebo $(n=8,61.5 \%)$. Both treatment interventions showed significantly lowered insulin secretion compared to placebo. 
Conclusion: Empagliflozin as well as Anakinra lowered glucose-requiring hypoglycemic episodes in patients after Roux-Y-gastric bypass by decreased insulin secretion. Therefore, empagliflozin may be a promising novel therapeutic option for patients with refractory postprandial hypoglycemia.

[1] Salehi et al. J Clin Endocrinol Metab 103: 2815-2826, 2018

[2] Dror E, et al. Nat Immunol. 2017 Mar;18(3):283-292.

\section{PS114 \\ An important role of GDF15 in inflammation-mediated podocyte dysfunction: an in vivo model of Minimal Change Disease in GDF15-deficient mice.}

Ekaterina von Rauchhaupt'; Mohsen Honarpisheh'; Vivian Würf'; Paulina Koehler'; Joanna Koziel' ${ }^{2}$; Maja Lindenmeyer ${ }^{3}$; Maciej Lech ${ }^{1}$

${ }^{1}$ Klinikum der Universität München, LMU München, Medizinische Klinik und Poliklinik IV, Munchen, Germany; ${ }^{2}$ Jagiellonian University, Krakow, Poland, Departments of Microbiology, Faculty of Biochemistry, Biophysics, and Biotechnology, Krakow, Poland; ${ }^{3}$ UKE Hamburg, III. Medizinische Klinik und Poliklinik, Hamburg, Germany;

Purpose: Growth differentiation factor-15 (GDF15) is a member of the TGF- $\beta$ cytokine superfamily that was shown to be elevated in patients with chronic kidney disease (CKD) and is associated with CKD progression. Encouraged by our observation that GDF15 is highly expressed in podocytes, we investigated the role of GDF15 in these cells using the puromycin aminonucleoside (PAN)-induced model of minimal change disease (MCD) both in-vivo and in vitro. We hypothesized that GDF15 may be an important factor of podocyte pathophysiology and study whether GDF15-mediated signaling affects the inflammatory responses as well as TGF- $\beta$ signaling in podocytes.

Methods: Puromycin aminonucleoside (PAN)-induced model of minimal change disease (MCD).

Results: Biochemical renal function indicators including serum BUN, creatinine and urine protein showed no clear phenotype upon PAN injection. Our in vivo experiments show that gender influences the renal phenotype of GDF15-deficient animals. We observed decreased albuminuria in female mice 3 days after PAN injection. Expression of chemokines such as $\mathrm{Cxcl}_{1}$ or $\mathrm{Ccl}_{2}$ increased in the GDF15-deficient PAN-treated males but not in females, confirmed that anti-inflammatory function of GDF15 might be gender-specific. Surprisingly, levels of GDF15 did not differ between males and females. Moreover, number of glomerular macrophages, neutrophils and T cells did not show significant differences between wild-type and GDF15-deficient mice upon PAN injection. Cultured podocytes constitutively expressed significant levels of GDF15 mRNA and its expression was decreased by PAN and upon stimulation with LPS. In vitro, pre-incubation with recombinant GDF15 of podocytes did not confirmed our in vivo findings. Oxidative stress responses in podocytes and chemokine production associated with PAN-induced injury were not affected by GDF15 pretreatment.

Conclusions: We conclude that GDF15 could potentially regulate the chemokine release pattern and the effects of GDF15 might be gender-specific. We suggest that podocyte-derived GDF15 may play role in glomerular disorders, but perhaps only by paracrine-acting on non-podocyte glomerular cells.

\section{PS115}

Prospective analysis of immune reconstitution in patients after Alemtuzumab-mediated $\mathrm{T}$ cell depletion as predictive marker for acute GVHD

\section{Saskia Hendriks'; Pascal Wölfinger ${ }^{2}$; Diana Kriege ${ }^{2}$; Eva-Maria Wag-} ner-Drouet ${ }^{2}$; Matthias Theobald ${ }^{2}$

'Universitätsklinik Mainz, Hämatologie, Pneumologie und internistische Onkologie, Mainz, Germany; ${ }^{2}$ Universitätsklinikum Mainz, Hämatologie, Pneumologie und interdisziplinäre Onkologie, Mainz, Germany

Introduction: Allogeneic hematopoietic stem cell transplantation (HSCT) is for many patients suffering from AML the only curative treatment option. One major complication is graft versus host disease (GvHD), caused by donor immune cells attacking healthy tissue.

Regulatory T cells (Treg) have been getting huge attention during the past years because of their important role in maintaining immune balance. Here we collected peripheral blood samples from 11 patients at different time points after HSCT to investigate immune-reconstitution of Treg as predictive marker for the development of GvHD.

Methods: We collected blood samples from 11 patients in the course of allogeneic HSCT prospectively once a week from $d+7$ up to $d+200$. All patients received conditioning regimen with Fludarabine and Melphalan, combined with Alemtuzumab for T cell depletion. 10 patients developed acute GVHD in the later course.

After isolation of PBMC's we performed FACS multicolor staining of $T$ cell and NK cells. Treg were identified as $\mathrm{CD}_{3}+\mathrm{CD}_{4}+\mathrm{CD}_{25}++$ Foxp3+, NK cells were characterized as CD3negCD56+CD16+ and divided in NK cell subpopulation due to their expression of $C_{5} 56 \mathrm{dim}$ or $\mathrm{CD}_{56} 6 \mathrm{high}$.

\section{Results:}

1. CD52neg T cells: All patients developing acute GVHD in the later course showed significant elevated levels of $C_{4}+C_{5}$ 2neg $T$ cells, especially CD52neg Treg at $d+50$.

2. $\mathrm{CD}$ 52neg Treg / CD8+ CD52+T cells: One patient not developing acute GVHD showed lots of CD52neg Treg but missed CD8+CD52+ effector $T$ cells. We recently showed that CD8+CD52neg effector $T$ cells are of impaired effector function. These data suggest that CD52neg Treg are only of relevance combined with functional CD8+CD52+ effector T cells in the development of aGVHD.

3. T cell marker: The patient without aGVHD showed elevated expression of Garp on Treg. Garp was significantly higher expressed on $\mathrm{CD}_{52}+$ Treg, indicating a better suppressive capacity of $\mathrm{CD}_{52}+$ Treg. This was detected throughout from $d+50$ until $d+200$. Tigit and ILT3 showed a heterogeneous expression profile without significant differences between the two groups.

5. NK cells: We detected a higher ratio of CD65++/CD56dim NK-cell population in the patient without GVHD. We could also show that Tigit is mainly expressed on CD56dim NK cells.

Discussion: Recently we presented Data on impaired suppressive capacity of $C_{52 n e g}$ Treg and the association with acute GVHD retrospectively (Wölfinger EBMT 2018, ASH 2017). Here we provide prospective Data on patients after the use of Alemtuzumab in the context of HSCT: Our preliminary data suggest that the total amount of CD52negTreg and the ratio of $C D 52$ neg Treg to $C D 8+C D 52+$ Treg on $d+50$ after allogenic HSCT could predict aGvHD. This data may be a basis for immune monitoring of patients at $d+50$ to evaluate their risk for aGVHD and could lead to the use of prophylactic Treg DLI in the context of Alemtuzumab mediated T cell depletion. 
PS116

\section{Außergewöhnliche Ursache einer sich rapide entwickelnden Dysphagie}

Katharina Schulz ${ }^{1}$; Thorsten Austein ${ }^{2}$; Annemone Finck'; Andreas Radeloff ${ }^{3}$

${ }^{1}$ St. Bernard Hospital in Brake, Medizinische Klinik I-Abteilung für Allgemeine Innere Medizin, Kardiologie und Gastroenterologie, Brake, Germany; ${ }^{2} \mathrm{St}$. Bernhard Hospital in Brake, Medizinische Klinik II - Abteilung für Hämatologie und internistische Onkologie, Brake, Germany; ${ }^{3}$ Evangelisches Krankenhaus Oldenburg, Universitätsklinik für Hals-Nasen-Ohren-Heilkunde, Kopf- und Hals-Chirurgie, Plastische Operationen, Oldenburg, Germany; ${ }^{4}$ St. Bernard Hospital in Brake, Medizinische Klinik II - Abteilung für Hämatologie und internistische Onkologie, Brake, Germany

Einleitung: Die Dysphagie gilt als Warnsymptom und tritt bei diversen benignen und malignen Erkrankungen auf. Häufige Ursachen einer Dysphagie sind Refluxösophagitis, eosinophile Ösophagitis, Achalasie sowie Ösophagus- und Kardiakarzinome $(1,2)$. Non-Hodgkin-Lymphome treten nur sehr selten extranodal auf, manifestieren sich dann aber an ungewöhnlichen Lokalisationen (3). Eine durch Lymphombefall des Rachenrings induzierte Dysphagie ist extrem selten (4). Wir berichten über die sehr seltene Erstmanifestation eines hoch-malignen Non-Hodgkin-Lymphoms an der Tonsilla palatina. Casereport: Ein bis dato gesunder 51-jähriger Mann stellte sich im Sommer 2018 wegen einer seit zwei Wochen progredienten Dysphagie vor. Die Racheninspektion zeigte eine pflaumengroße Schwellung der rechten Tonsille mit zentraler Ulzeration (Abb.1). Eine B- Symptomatik wurde verneint. Zervikale Lymphknotenschwellungen fanden sich nicht. Der weitere körperliche Untersuchungsbefund war unauffällig. Die routinemäßige Labordiagnostik erbrachte keinen pathologischen Befund. Bei unklarer hyperplastischer, ulzeröser Tonsille erfolgte eine Panendoskopie mit Probebiopsie der vergrößerten Tonsille. Der histopathologische Endbefund erbrachte eine Infiltration der Tonsille durch ein diffus großzelliges B-Zell-Lymphom (GCB-Subtyp ohne MYC-Bruch, Immunhistochemisch-CD20 positiv).

Nach Überweisung des Patienten in unsere onkologische Abteilung erfolgte die CT- morphologische Komplettierung des Stagings (Hals $\mathrm{CT}$, Thorax CT, Abdomen CT) sowie Beckenkammhistologie. Hinweise für eine weitere Lymphommanifestation ergaben sich weder radiologisch noch in der Knochenmarkshistologie. Bereits nach drei Zyklen der kombinierten Immun- und Chemotherapie mit Rituximab, Cyclophosphamid, Doxorubicin, Vincristin, Prednisolon (R-CHOP-21 Schema) plus Neutropenie-Prophylaxe mit peg-Filgrastrim (Neulasta ${ }^{\circledR}$ ) zeigte sich eine vollständige Remission (Abb. 2). Nach insgesamt 6 Zyklen $\mathrm{R}-\mathrm{CHOP}$ und zweimaliger Monotherapie mit Rituximab wurde die Therapie beendet. Nennenswerte therapieassoziierte Nebenwirkungen traten nicht auf. Die Therapie konnte problemlos ambulant durchgeführt werden. Die Nachsorge blieb bisher ohne Hinweis eines Rezidivs. Schlussfolgerung: Bei rasch wachsenden Tonsillen sollte neben der Möglichkeit eines Karzinoms immer an die Möglichkeit einer lymphatischen Erkrankung gedacht und aufgrund der grundsätzlich unterschiedlichen Therapiestrategien (Operation versus Chemotherapie) umgehend eine Biopsie zur Klärung der Dignität durchgeführt werden.

[1] Torresan F, Ioannou A, Azzaroli F, Bazzoli F.: Treatment of achalasia in the era of high-resolution manometry. Ann Gastroenterol. 2015; 28: 299-306.

[2] Inoue H, Kaga M, Ikeda H, et al.: Magnification endoscopy in esophageal squamous cell carcinoma:a review of the intrapapillary capillary loop classification. Ann Gastroenterol. 2015; 28:41-48.
[3] Austein T, Demme B: Uncommon recurrence of follicular lymphoma. European Journal of Haematology, 2012, 88:91

[4] Mohammadianpanah M, Omidvai S, Mosalei A, et al. Treatment results of tonsillar lymphoma: 10 years experience. Ann Hematol. 2005; 83:223-226.

\section{PS117 \\ Identifizierung und Validierung eines T Zell Biomarkers für Graft-versus-Host-Disease (GvHD) bei Patienten nach allogener hämatopoetischer Stammzelltransplantation (allo-HSZT)}

Samira Reuß'; Beate Hauptrock'; Matthias Theobald'; Hakim Echchannaoui'; Eva Wagner-Drouet ${ }^{1}$; Diana Kriege'; Leonie Schmidt ${ }^{2}$

'Universitätsmedizin Mainz, III. Medizinische Klinik, Mainz, Germany; ${ }^{2}$ Universitätsmedizin Mainzrsi, III. Medizinische Klinik, Mainz, Germany

GvHD ist nach dem Rezidiv die häufigste Komplikation nach einer allo-HSZT und kann in einer chronischen und einer akuten Form auftreten, welche beide mit einer höheren Mortalität einhergehen. Derzeit stützt sich die Diagnose auf die klinische Symptomatik und die Histologie; ein Biomarker zur Diagnose des frühen Auftretens von GvHD gibt es gegenwärtig nicht. Daher untersuchten wir verschiedene $T$ Zell Marker und beobachteten eine niedrige Zelloberflächenexpression von TIGIT (T cell immunoreceptor with Ig and ITIM domain) auf den $\mathrm{CD}_{3}+\mathrm{T}$ Zellen aus peripherem Blut von Patienten mit chronischer GvHD (cGvHD). TIGIT ist ein inhibitorisches co-stimulatorisches Molekül, das auf aktivierten CD4+ und CD8+T Zellen, auf regulatorischen T Zellen (Tregs), T Gedächtniszellen, follikulären T Helferzellen und natürlichen Killerzellen vorkommt. Die immunmodulatorische Funktion von TIGIT wir durch Bindung an den Poliovirus Rezeptor (PVR) Ligand (CD155) initiiert. Eine hohe TIGIT Expression ist mit der Dysfunktion der Effektor T Zellen assoziiert, während in Tregs die suppressive Funktion verstärkt wird.

In unserer Studie untersuchten wir die mononukleären Zellen des peripheren Bluts (PBMCs) von 76 Patienten nach allo-HSZT. Die Proben wurden uns durch die Knochenmarktransplantationseinheit der Universitätsmedizin Mainz zur Verfügung gestellt: 33 ohne GvHD, 31 mit cGvHD und 12 mit akuter GvHD (aGvHD). Die Patienten waren hauptsächlich an AML erkrankt, andere Erkrankungen umfassten MDS, ALL, $\mathrm{CML}$, aplastische Anämie und CLL. Nach Ficoll-Dichte-Zentrifugation wurden die PBMCs mit Antikörpern gegen TIGIT, CD3, CD4 und CD8 gefärbt und mittels Durchflusszytometrie analysiert.

Beim Vergleich von Patienten mit cGvHD und keiner GvHD fanden wir ein signifikant niedrigeres TIGIT Level bei Patienten mit cGvHD $(p=0.0081)$. Im Gegensatz dazu scheint die TIGIT Expression bei Patienten mit aGvHD höher zu sein als bei cGvHD, jedoch war dieser Unterschied nicht signifikant. Die Korrelation der TIGIT Expression und cGvHD wurde insbesondere für die männlichen Empfänger unabhängig vom Spendergeschlecht beobachtet. Des Weiteren untersuchten wir, ob sich die TIGIT Expression in der CD4+ und CD8+ Fraktion unterscheidet und fanden, dass die Expression bei den CD8+ T Zellen deutlich höher ist als bei den CD4+ T Zellen. Erstaunlicherweise war cGvHD ausschließlich mit der TIGIT Expression in der Untergruppe der CD8+ T Zellen assoziiert. Die Intensität des Konditionsregimens (myeloablativ; reduzierte Intensität) korrelierte nicht mit dem Level der TIGIT Expression.

Obwohl der Unterschied der TIGIT Expression und cGvHD hauptsächlich bei männlichen Patienten beobachtet wurde, ist die Auswertung einer größeren Patientenkohorte notwendig, um diese Daten zu validieren. Außerdem deutet die signifikante Korrelation im CD8+ 
Kompartiment auf die potentielle Rolle dieser Zellen im Auftreten der cGvHD hin.

Yu Xin et al. The surface protein TIGIT suppresses T cell activation by promoting the generation of mature immunoregulatory dendritic cells. Nature Immunology 10, 48-57 (2009).

Kurtulus Sema et al. TIGIT predominantly regulates the immune response via regulatory $T$ cells. The Journal of Clinical investigation 125 , 4053-4062 (2015)

Johnston R.J. et al. The Immunoreceptor TIGIT Regulates Antitumor and Antiviral CD8+ T Cell Effector Function. Cancer Cell 26, 923-937 (2014)

\section{PS118}

\section{Disseminated Nocardia infection presenting as multi-organ infection in a woman with systemic lupus erythematosus}

\section{V.S. Srikanth; Divakar Arun}

Amrita Institute of Medical Sciences and Research Centre, Internal Medicine, Kochi, Kerala, India

Background: Nocardia is an opportunistic pathogen which can cause disseminated infection in an immunocompromised host. The most common type of skin lesion reported with disseminated Nocardia is a subcutaneous nodule. Long term corticosteroid treatment is one of the largest risk factors for developing disseminated Nocardia. Initial treatment is empiric as each strain has unique susceptibilities and it takes weeks to speciate and test sensitivities. Pulmonary lesions and brain lessons are manifestations of Nocardia in a SLE patient

Main observations: A 33-year-old female on long term corticosteroids for systemic lupus erythematosus (SLE) with lupus nephritis presented with pain, tenderness and multiple abscess in the hand and leg CT-scan revealed involvement of the lungs in left middle lobe and brain, in the frontal lobe. The patient also had multiple lesions in the retina, the patient improved after supportive treatment. CT revealed abscess and pus aspirated and culture showed no growth. She had eye pain, blurring of vision. Ophthalmology evaluation showed multiple sub retinal abscess and vitritis of right eye and 2 retinal abscess in in the left eye. Blood culture grew Nocardia nova. She was given intravitreal Imipenem. She was managed as a case of disseminated Nocardiosis with Meropenem, Septran along with other supportive measures. Ophthalmology opinion was sought, she was advised to be started on Imipenem injection

USG abdomen: Both kidneys shows mildly raised echotexture with maintained corticomedullary differentiation, Mild ascites, Liver shows few calcified specks as described Calcified granulomas.

Upper Gl endoscopy - Reflux Esophagitis LA - A. Antral gastritis. CLO negative Peripheral blood smear (3/11/16) - Normocytic normochromic anaemia with neutrophilia, leucocytosis, thrombocytopenia with suspicious schistocytes ECHO (4/11/16) - Concentric LVH No RWMA Good LV systolic function E \& A fused, Grade I diastolic dysfunction. Independent mobility suggestive of active vegetation or nonbacterial thrombotic endocarditis noted in TTE.

Conclusions :This case of Nocardiosis had unusual cutaneous, pulmonary, brain and retinal findings that could have misguided the clinician, but the blood culture and Gram stain proved to be useful for rapid diagnosis and proper treatment. Nocardiosis has been increasingly recognized in SLE. Although still uncommon, it is an important opportunistic infection because it is curable and mortality is usually caused by delay in diagnosis and treatment. A high index of suspici- on, an aggressive approach to diagnosis, and early empirical therapy are essential principles of management. Nocardial infection, though rare in SLE has a good outcome. Involvement of brain, skin and lung together in a patient with background of immunosuppression should raise a suspicion of nocardial infection.

[1] Dodiuk-Gad R, Cohen E, Ziv M, Goldstein LH, Chazan B, Sha- fer J, Sprecher $\mathrm{H}$, Elias M, Keness $\mathrm{Y}$, Rozenman D. Cutane- ous nocardiosis: report of two cases and review of the litera- ture. Int J Dermatol. 2010; 49: 1380-1385. PMID: 21155087

[2] Wong KM, Chak WL, Chan YH, Choi KS, Chau KF, Lee KC, Li CS. Subcutaneous nodules attributed to nocardiosis in a renal transplant recipient on tacrolimus therapy. Am J Ne- phrol. 2000; 20: 138-141. PMID: 10773614.

[3] Shapiro PE, Grossman ME. Disseminated Nocardia asteroids with pustules. J Am Acad Dermatol. 1989; 20: 889-892. PMID: 2654222.

[4] George SJ, Rivera AM, Hsu S. Disseminated cutaneous Nocardiosis mimicking cellulitis and erythemanodosum. Dermatol Online J. 2006; 12: 13. PMID: 17459299.

[5] Hara H, Wakui F, Ochiai T. Disseminated Nocardia farcinica infection in a patient with systemic lupus erythematosus. J Med Microbiol. 2011; 60: 847-850. PMID: 21372184.

[6] Justiniano M, Glorioso S, Dold S, Espinoza LR. Nocardia bra- in abscesses in a male patient with SLE: successful outcome despite delay in diagnosis. Clin Rheumatol. 2007; 26: 1020-1022. PMID: 16683177.

[7] Leong KP. Tee NW, Yap WM, Chee TS, Koh ET. Nocardiosis in patients with systemic lupus erythematosus. The Singapore Lupus Study Group. J Rheumatol. 2000; 27: 1306- 1312. PMID: 10813308.

[8] Soy M, Oktun MT, Tunçbilek N, Ermantaş N, Okten O, Altinay G, Turgut B. Nocardiosis in a patient with primary anti-phospholipid syndrome. Rheumatol Int. 2006; 26: 461-464. PMID: 16404563.

[9] Carducci M, Nosotti L, Calcaterra R, Bonifati C, Mussi A, Pe- lagalli L, Di Emidio L, Laurenzi L, Russo A, Franco G, Toma L, Morrone A. Early development of disseminated nocardiosis during immunosuppressive treatment for pemphigus vulga- ris. Eur J Dermatol. 2007; 17: 346347. PMID: 17540653.

[10] Kakurai M, Hiraga T, Yamada T, Usui K, Kiyosawa T, Naka- gawa H. Subcutaneous nocardial abscesses in a patient with bullous pemphigoid during immunosuppressive therapy: re- port of a case and review of the Japanese literature. J Der- matol. 1999; 26: 829-833. PMID: 10659507

[11] Arora G, Friedman M, Macdermott RP. Disseminated Nocardia nova infection. South Med J. 2010; 103: 1269-1271. PMID: 21037519 


\section{A}

Abdollahi, A. (Heidelberg), PS005 Acar, H. (Düsseldorf) , PS009 Afifi, A. (Magdeburg) , PS013 Ahmad, S. (Göttingen) , PS047 Ajayi, S. (Freiburg) , YIA05 Al Ali, R. (Saarbrücken) , PS099 Albrecht, H. (Nürnberg), PS061, PS062

Alter, P. (Marburg) , PS044, PS086, PS087, PS088, YIA04

Amer, W. (Köln) , PS012

Anders, H. (München) , PS026 Andersen, J. (Kopenhagen) , PS012 Anneken, A. (Erlangen) , PS061 Appenrodt, B. (Homburg) , YIA03 Arends, W. (Tutzing) , PSO03 Arnold, L. (Essen) , YIA08 Arrivas, M. (Aachen) , PS079, YIA02 Arslanow, A. (Mainz) , PS104 ARUN, D. (Kochi, Kerala) , PS118 Aschenbrenner, E. (Regensburg), PS010, PS016, PS017, PS018, PS059, YIA06 Assmuth, S. (Essen) , PS015 Austein, T. (Brake) , PS116 Aykara, I. (Bad Nauheim) , PS051, PS064

\section{B}

Back, D. (Liverpool) , PS040 Backu, E. (Regensburg) , PS017 Baenkler, H. (Erlange) , PS024 Balavarca, Y. (Heidelberg) , YIA01 Balermpas, P. (Frankfurt) , PS005 Bals, R. (Homburg) , PS044, PS088, YIA04

Batista, J. (Aachen) , PS023

Bauer, L. (Homburg) , PS048

Baum, K. (Köln) , PS090, PS091 , PS092 Baumann, M. (Heidelberg) , PS005

Bechmann, L. (Magdeburg) , PS015

Beck, S. (Kiel) , PS039

Becker, D. (Mainz) , PS020

Becker, I. (Köln) , PS066

Beeres, M. (Frankfurt am Main) , PS109

Behr, J. (Munich) , PS082, PS086, YIA04 Behr, J. (München),

Belka, C. (Munich) , PS005

Belling, V. (Stuttgart) , PS110

Bengel, P. (Göttingen) , PS047

Benignus, C. (Stuttgart) , PS110

Benzing, T. (Köln) , PS066

Berchtold, S. (Tübingen) , PS058

Best, J. (Magdeburg) , PS015

Bester, J. (Mainz) , PS084, PS105

Biegel, E. (Lüdenscheid) , PS108

Biener, M. (Heidelberg) , PS079

Bieniek, Z. (Berchtesgaden) , PS097

Biertz, F. (Hannover) , PS044, YIA04

Binder, H. (Freiburg / Mainz) , PS104, PS012

Blankart, R. (Bern) , PS008

Blankenberg, S. (Hamburg), PS088
Böhm, M. (Homburg) , PS028, PS043, PS048, PS056, PS060, PS069, PS099 Bollheimer, C. (Aachen) , PS023 Böni-Schnetzler, M. (Basel) , PS113 Boor, P. (Aachen) , PS026 Botsios, S. (Lüdenscheid) , PS108 Bott, N. (Köln) , PS090, PS091, PS092 Bozec, A. (Erlangen) , PS065 Bramlage, P. (Cloppenburg) , PS063 Brenner, H. (Heidelberg), YIA01 Brenner, T. (Heidelberg) , PS074 Brinkkötter, P. (Köln) , PS066 Brodehl, A. (Bad Oeynhausen), YIA07

Brodesser, S. (Köln) , PS015

Bruensing, J. (Aachen) , YIA02 Brunner - La Rocca, H. (Maastricht) ,PS006

Brütting, A. (Erlangen) , PS061 Bucher, A. (Frankfurt am Main), PS109

Budach, V. (Berlin) , PS005

Burst, V. (Köln) , PS066

Butuk, D. (Meridian) , PS103

C

Canbay, A. (Magdeburg) , PS013, PS015

Carpinteiro, A. (Essen) , YIA08

Casper, M. (Homburg) , YIA03

Castven, D. (Mainz) , PS020

Castven, C. (Mainz) , PS012

Chehab, G. (Düsseldorf) , PS009

Chen, Y. (Essen) , YIA08

Chen, M. (Corvallis) , PS103

Chlibek, R. (Hradec Kralove) , PS100

Christiansen, M. (Köln) , PS066

Clarnette, R. (Perth) , PS071

Combs, S. (Munich) , PS005

Cornberg, M. (Hannover) , PS040

Cornely, F. (Köln) , PS076

Cornely, O. (Köln) , PS076

Cunningham, A. (Sydney) , PS100

Curran, D. (Wavre) , PS103

Czauderna, C. (Mainz) , PS012, PS020

\section{D}

Dagnew, A. (Rockville) , PS100 Dahlem, G. (Gießen) , PS083 Debus, J. (Heidelberg) , PS005 Demuth, I. (Berlin) , PS042 DHAOUADI, R. (BIZERTE) , PS112 Didiasova, M. (Gießen) , PS085 Diebold, S. (Aachen) , PS073, YIA02 Diepolder, I. (Aachen) , PS073 Dierks, S. (Göttingen) , PS107 Diez Domingo, J. (Valencia) , PS100 Diller, M. (Bad Nauheim), PS049, PS051, PS064

Dittmann, S. (Münster) , YIA07 Donath, M. (Basel) , PS113
Dufke, A. (Tübingen) , PS068 Dührsen, U. (Essen) , YIA08 Durmaz, M. (Hof) , PS063 Duyster, J. (Freiburg), YIA05 Dybkova, N. (Göttingen) , PS047

\section{E}

Echchannaoui, H. (Mainz) , PS117 Eckert, R. (Esslingen) , PS081 Eder, T. (Berlin) , PS005 Eder, L. (Göttingen) , PS107 Eder, A. (Marburg) , PS087 Effenberger, J. (Berlin), PS004 El Idrissi, M. (Rixensart) , PS103 Emrich, I. (Homburg) , PS056 Emrich, I. (Homburg) , PS048, PS057 Engelhardt, M. (Freiburg), YIA05 Engelmann, T. (Mainz) , PS104 Ewen, S. (Homburg) , PS043, PS060 Eyermann, R. (München) , PS052, PS054, PS094, PS095, PS096, PS097

\section{F}

Faehling, M. (Esslingen), PS081 Fallscheer, S. (Esslingen) , PS081 Farber, M. (Essen) , YIA08 Farin-Glattacker, E. (Freiburg) , PS104

Fichtner, U. (Freiburg) , PS104 Ficker, J. (Nürnberg) , YIA04 Finck, A. (Brake) , PS116 Findeisen, H. (Münster) , PS073 Fink, T. (Ansbach) , PS029 Fink, C. (Darmstadt) , PS036 Finzel, S. (Freiburg) , PS025 Fischer, B. (Münster) , YIA07 Fischer, J. (Löwenstein) , PS029 Fisser, C. (Regensburg) , PS075 Fissette, L. (Rixensart) , PS103 Fleischer, V. (Berlin) , PS005 Fliser, D. (Homburg) , PS048, PS056, PS057

Fogarty, C. (Spartanburg) , PS103

Foldenauer, A. (Frankfurt am Main), PS079

Förster, K. (Dresden) , PS067 Franck, M. (Magdeburg) , PS031 Frank, J. (Tübingen) , PS067 Franz, W. (München) , PS004, PS008 Frenk, F. (Erlangen) , PS098 Frommer, K. (Bad Nauheim) , PS053, PS077

\section{G}

Galle, P. (Mainz) , PS012, PS020, PS104 Gamstätter, T. (Wiesbaden) , PS041 Ganster, C. (Göttingen) , PS107 Gao, Y. (Cork), PS071
Gappa, H. (Sankt Augustin) , PS009 Gazou, A. (Tübingen) , PS068 Geisel, J. (Homburg) , PS056 Gerken, G. (Essen) , PS015 Giannitsis, E. (Heidelberg) , PS079 Gisch, D. (Homburg) , PS104 Goekyildirim, M. (Gießen) , PS083 Goj, D. (Hamburg) , PS025 Gökhan, Y. (Mannheim) , PS045 Golubnitschaja, O. (Bonn) , PS006 Gorfinkel, I. (Toronto) , PS100 Gorgus, E. (Kaiserslautern) , PS038 Götze, T. (Erlangen) , PS024 Graf, E. (Freiburg) , PS104 Grandel, U. (Gießen) , PS083 Grandt, D. (Saarbrücken) , PS041 Greinert, R. (Halle) , YIA03 Greinert, O. (Köln) , PS032, PS034, PS035

Greulich, T. (Marburg) , PS087 Grimminger, P. (Mainz) , PS020 Grimminger, F. (Gießen) , PS083 Grosu, A. (Freiburg) , PS005 Groves, R. (Nürnberg) , PS055 Grupping, K. (Wavre) , PS103 Gschwendtner, H. (Regensburg)， PS010, PS059, YIA06

Gülow, K. (Regensburg) , PS010, PS017, PS018, PS059, PS080, YIA06

Gungl, A. (Graz) , PS085

Günyak, G. (Stuttgart) , PSO02

\section{H}

Haack, T. (Tübingen) , PS068 Haase, D. (Göttingen) , PS107 Habib, A. (Perth) , PS071 Haderer, M. (Regensburg), PS016, PS062, YIA06

Hagel, A. (Schwabach) , PS061, PS062 Hager, L. (Saarbrücken) , PS008 Hain, J. (München) , PS100, PS103 Haj-Yehia, E. (Aachen) , YIA02 Halim, C. (Aachen) , PS073 Haller, F. (Erlangen) , $\mathrm{PS} 024$ Hamberger, U. (Landsberg) , PS093 Hanoun, M. (Essen), YIA08 Härle, P. (Mainz) , PS084, PS105 Hartley, P. (Uniontown) , PS103 Hartmann, M. (Mainz) , PS014 Hartmann, A. (Erlangen) , PS024 Hartmann, B. (Ludwigshafen) , PS063

Hasenfuß, G. (Göttingen) , PS047 Hasseli, R. (Bad Nauheim) , PS051 Hasslinger-Pajtler, F. (Frankfurt/ Mainz) , PS067

Hattar, K. (Gießen) , PS083 Hauptrock, B. (Mainz) , PS117 Hees, A. (Köln) , PS066 Heil, F. (Andernach) , PS104 Heilmann-Heimbach, S. (Bonn), PS012

Heine, G. (Frankfurt) , PS048, PS056, PS057 
Heinemann, A. (Graz) , PS057 Heinemann, T. (King of Prussia), PS100

Heinrich, J. (Erlangen) , PS025 Heinrichs, L. (Mainz) , PS096 Hellwege, J. (Nashville) , YIA01 Helms, T. (Berlin) , PS001, PS006 Hendriks, S. (Mainz) , PS115 Hepprich, M. (Basel) , PS113 Herbig, B. (München) , PS086 Hernandez, A. (Freiburg) , PS067 Herr, C. (Homburg) , PS088 Herrmann, H. (Erlangen) , PS098 Herrmann, J. (Ulmenweg 18) , PS072 Herve, C. (Wavre) , PS100 Hess, A. (Berlin) , PSO05 Hillebrand, M. (Mainz) , PS084 Hinner, M. (Scotch Plains, New Jersey), PS093

Hoedemakers, T. (Sittard) , PS006 Holl, R. (UIm) , PS063 Hollstein, T. (Berlin) , PS030 Honarpisheh, M. (München) , PS114 Höner zu Siederdissen, C. (Hannover) , PS040

Hoog-Antink, C. (Aachen) , PS023 Hörning, A. (Erlangen) , PSO24 Hotfiel, K. (Erlangen) , PS025, PS062 Hoyer, S. (Nürnberg) , PS055 Huber, B. (Munich) , PS082 Hug, M. (Freiburg) , YIA05 Hülser, M. (Bad Nauheim/ Gießen), PS051, PS065

Hummel, M. (Berlin) , PS005 Hundsdorfer, J. (Homburg) , PSO48 Hupfer, S. (Nürnberg) , PS027, PS055 Hwang, S. (Taipei) , PS100

Idel, P. (Aachen) , PS073 Ikematsu, H. (Tokyo) , PS100

\section{J}

Jacobs, S. (Wiesbaden) , PS041 Jäger, J. (Homburg) , PS104 Jansky, M. (Mainz) , PS104 Johnson, R. (Bristol) , PS100 Jöhrens, K. (Berlin) , PS005 Jörres, R. (München) , PS044, PS086, PS088, YIA04

K

Kaestner, M. (Chemnitz) , PS095 Kahles, F. (Aachen) , PS073, PS079, YIA02

Kahnert, K. (München) , PS044, PS086, YIA04 Kälble, F. (Heidelberg) , PS046, PS074
Kalema, G. (Wavre) , PS100 Kälsch, A. (Mannheim) , PS106 Karakaya, S. (Stuttgart) , PS110 Karamanos, D. (Thessaloniki, Griechenland) , PSO70

Kassner, U. (Berlin) , PS030

Katmeh, L. (Homburg) , PS099

Katsouda, A. ( Lamia, Griechenland), PS070

Katus, H. (Heidelberg) , PS079

Kaul, S. (Essen/Düsseldorf) , PS067

Kayser, A. (Münster) , YIA07

Kelber, O. (Darmstadt) , PS032, PS033,

PS034, PS035, PS036, PS037, PS038

Kern, S. (Graz) , PS057

Khader, O. (Lüdenscheid) , PS108

Kim, D. (Heidelberg) , PS046

Klaußner, M. (Erlangen) , PS098

Kleber, M. (Mannheim) , PS045

Klein, N. (Oakland) , PS103

Kleist, C. (Heidelberg) , PSO46

Klemm, J. (Mainz) , PS014

Klepetko, W. (Wien) , PS085

Klinkhammer, B. (Achen) , PS026

Knaus, J. (Freiburg) , PS104

Kneidinger, N. (Munich) , PS082

Koch, A. (Aachen) , YIA02

Koehler, P. (Köln) , PS076

Koehler, F. (Köln) , PS076

Koehler, P. (München) , PS114

Kokelmann, C. (Dresden) , PS007

König, M. (Berlin) , PSO42

Königsdorfer, N. (München) , YIA04

Konschak, R. (Berlin), PS005

Kopp, M. (Esslingen) , PS081

Koppenhöhl, C. (München) , PS067

Kornek, S. (Berlin) , PSO04

Kosanovic, D. (Gießen) , PS085

Kovacs, Z. (Essen) , PS089

Koziel, J. (Krakow) , PS114

Kraft, K. (Rostock) , PS037

Kramberg, S. (Esslingen) , PS081

Krämer, B. (Mannheim) , PS106,

PS045

Krause, M. (Dresden), PS005

Kressel, J. (Erlangen), PS062

Kriege, D. (Mainz) , PS115, PS117

Krischke, E. (Erlangen) , PSO62

Krischke, F. (Erlangen), PS062

Kuehbacher, T. (Hamburg) , PS039

Kunst, C. (Regensburg), PS010,

PS016, PS017, PS059, YIA06

Kunz, A. (Heidelberg) , PS046

Küppers, D. (Bad Nauheim) , PS050

Kwapiszewska, G. (Graz) , PS085

L

Lachmund, T. (Magdeburg) , PS019

Lal, H. (King of Prussia), PS100

Lallah Missimana, P. (Bad Nau-

heim), PS049

Lammert, F. (Homburg), PS104,

YIA03

Lampey, J. (Gießen) , PS083
Lang, H. (Mainz) , PSO20

Langhof, H. (Berchtesgaden), PS095, PS096, PS097

Langmann, A. (München), YIA09

Langmann, P. (Karlstadt) , YIA09

Lanzinger, S. (UIm) , PS063

Lauder, L. (Homburg) , PS043, PS060

Lauer, U. (Tübingen) , PS058

Laurentius, T. (aachen) , PS023

Lebherz, C. (Aachen) , PS073, PS079, YIA02

Lech, M. (München) , PS114

Lehner, M. (München) , PS008

Lehrke, M. (Aachen) , PS073, PS079,

YIA02

Leikeim, L. (Mannheim) , PS106

Leonhardt, S. (Aachen), PS023

Lerzer, C. (Donaustauf) , PS075

Leschke, M. (Esslingen) , PS081

Leuschner, G. (Munich) , PS082

Levin, M. (Aurora) , PS100, PS103

Liapis, H. (Washington), PS026

Liebe, R. (Homburg) , YIA03

Liersch, S. (Berlin), PSO04

Lindenmeyer, M. (Hamburg) , PS114

Linge, A. (Dresden) , PS005

Link, A. (Magdeburg) , PS019, PS031

Lohaus, F. (Dresden) , PS005

Lorenz, H. (Heidelberg) , PS046

Lubnow, M. (Regensburg), PS075

Lucke, T. (München) , YIA04

Lugano, F. (Berlin), PSO42

Luiz, T. (Kaiserslautern) , PS001

Luo, Y. (Erlangen) , PS065

Lutter, J. (Neuherberg) , PS086, YIA04

Lutter, J. (München), Gleiche Per. Unterschiedliche Orte

M

M GOPALA KRISHNA, P. (Kochi,

Kerala), PS021, PS022, PS101

Maasoumy, B. (Hannover) , PS040

Madisch, A. (Hannover) , PS036

Mager, S. (Marburg) , PS087

Magnussen, C. (Hamburg) , PS088

Mahfoud, F. (Homburg), PS028,

PS043, PS060, PS099

Maier, L. (Regensburg) , PS047

Maier-Stocker, C. (Regensburg),

PS080

Mainz, D. (Andernach) , PS104

Malfertheiner, P. (Magdeburg),

PS019, PS031

Manns, M. (Hannover) , PS040

Marczewski, T. (Kassel) , PS029

Marquardt, J. (Mainz) , PS012, PS014,

PS020

Marra, F. (Liverpool) , PS040

Marsche, G. (Graz) , PS056, PS057

Martin, L. (Aachen) , YIA02

Martin, R. (Göttingen) , PS107

Martin, S. (Baltimore, Maryland),

PS056

Marx, N. (Aachen) , PS073, PS079,
YIA02

März, W. (Mannheim) , PS045

Massberg, S. (Munich) , PS082

Matthews, S. (Wavre) , PS103

Mavromanoli, A. (Thessaloniki,

Griechenland), PS070

Mayerhofer, B. (München) , PS044

McElhaney, J. (Sudbury) , PS100

McNeil, S. (Halifax) , PS100

Meiß, F. (Freiburg), YIA05

Mellinghoff, S. (Köln) , PS076

Merkel, T. (Nürnberg) , PS027

Merle, U. (Heidelberg) , PS074

Mertens, R. (Aachen) , PS073, PS079,

YIA02

Messerschmidt, I. (Essen) , PS015

Mester, P. (Regensburg) , PS010,

PS111

Meyer, A. (Köln) , PS066

Michalski, M. (Regensburg) , PS018

Milger, K. (Munich) , PS082

Millenaar, D. (Homburg) , PS069

Milting, H. (Bad Oeynhausen), YIA07

Moellmann, J. (Aachen) , PS079,

YIA02

Moleda, L. (Regensburg) , PS080,

PS111

Möller, J. (Darmstadt) , PS033, PS038

Möllmann, J. (Aachen) , PS073

Möllmann, M. (Essen) , YIA08

Molloy, W. (Cork) , PS071

Morath, C. (Heidelberg) , PS046,

PS074

Mösges, R. (Köln) , PS032, PS034,

PS035

Mrkvan, T. (Wavre) , PS100

Mross, T. (Heidelberg) , PS071

Mulay, S. (München) , PS026

Müller, T. (Regensburg) , PS075

Müller, F. (Köln) , PS066

Müller-Ladner, U. (Bad Nauheim/

Gießen) , PS049, PS051, PS053, PS064,

PS065, PS077

Müller-Schilling, M. (Regensburg),

PS010, PS016, PS017, PS018, PS059,

PS080, PS111, YIA06

Munker, D. (Munich) , PS082

N

Nagel, M. (Mainz) , PS104

Neumann, E. (Bad Nauheim) , PS049,

PS051, PS053, PS064, PS077

Neumann, J. (Hamburg) , PS088

Neumann, E. (Gießen) , PS065

Neurath, M. (Erlangen), PS072, PS098

Neurohr, C. (Gerlingen) , PS082

Nevares, M. (Colorado Springs),

PS103

Nguyen-Tat, M. (Osnabrück) , PS104

Nicholas, P. (Cork) , PS071

Nieber, K. (Leipzig) , PS032, PS033,

PS038

Nieber, K. (Bonn) , PS034, PS035

Nieber, K. (Leipzig) , PS037 
Nitsch, T. (Passau) , PS011 Noetzel, N. (Köln) , PS066 Nusshag, C. (Heidelberg) , PS046, PS074

\section{0}

O Caoimh, R. (Galway) , PS071

Odenthal, M. (Köln) , PS012

Öhmichen, S. (Chemnitz) , PS095

Oostvogels, L. (Wavre) , PS100, PS103

Ortner, J. (Mainz) , PS104

O'Rourke, C. (Kopenhagen) , PS012

Pabel, S. (Regensburg), PS047

Palant, A. (Berlin) , PS006

Park, Y. (Seoul) , PS012

Pauksens, K. (Uppsala) , PS100

Pavlicek, V. (Homburg) , PSO69

Pereira, S. (Mainz) , PS020

Petrovic, A. (Gießen) , PS085

Pfeifer, M. (Regensburg) , PS075

Pfrogner, R. (Homburg) , PS069

Konsortium PICASO, (Sankt

Augustin), PS009

Pickert, L. (Köln) , PS066

Pigorsch, S. (Munich) , PS005

Pilotto, A. (Genova) , PS066

Polidori, M. (Köln) , PS066

Pollinger, K. (Regensburg) , PS010,

PS016, PS017, PS018, PS059

Poplawski, A. (Mainz) , PS012

Port, K. (Hannover) , PS040

Porthun, M. (Hamburg) , PSO39

\section{R}

Rabbe, S. (Hamburg) , PS008 Rabe, K. (Grosshansdorf) , PS088 Rabini, S. (Darmstadt) , PS036 Radeloff, A. (Oldenburg) , PS116 Raithel, M. (Erlangen) , PS024, PS025, PS061, PS062

Rarek, M. (Köln) , PS066

Raskopf, E. (Köln) , PS032, PS034, PS035

Rautenberg, B. (Freiburg) , YIA05 Razouk, A. (Homburg) , PS069 Rechenauer, T. (Erlangen) , PS024 Rehart, S. (Frankfurt) , PS049, PS051, PS053, PS064, PS077

Reichert, M. (Homburg) , YIA03 Reil-Held, A. (Heidelberg) , PS067 Reinhardt, H. (Freiburg), YIA05 Reljic, D. (Erlangen) , PS098 Reljic, D. (Ulmenweg 18) , PS072 Renoy, H. (Kochi, Kerala) , PS102 Reuß, S. (Mainz) , PS117 Richter, J. (Düsseldorf) , PS009
Ricken, E. (Düsseldorf) , PS009 Ridder, D. (Mainz) , PS012 Rieder-Nelissen, C. (Mainz) , PS084 Rief, W. (Marburg) , PS086 Rieker, R. (Erlangen) , PS025 Rieß, O. (Tübingen) , PS068 Ripoll, C. (Halle) , YIA03 Ritter, B. (Kassel) , PS029 Rittscher, K. (Göttingen) , PS107 Rogacev, K. (Wismar) , PS056, PS057 Rosania, R. (Magdeburg), PS013 Rösner, A. (Freiburg) , YIA05 Ruch, M. (Freiburg) , YIA05 Rückbeil, M. (Aachen) , PS079 Rückel, A. (Erlangen) , PS024 Rudofsky, G. (Olten) , PS113 Rupp, C. (Heidelberg) , PS074

\section{$s$}

Sandoval, D. (Ann Arbor) , YIA02 Sauermilch, H. (Gießen), PS065 Schaab, R. (Göttingen) , PS107 Schäfer, L. (Frankfurt) , PS085 Schäfer, H. (Freiburg) , YIA05 Schaier, M. (Heidelberg) , PS046 Scharnagl, H. (Graz) , PS056 Schelker, B. (Basel) , PS113 Scherm, S. (Regensburg) , PS016 Schermuly, R. (Gießen) , PS085 Schett, G. (Erlangen) , PS065 Scheubeck, G. (München) , PS058 Scheuer, A. (Homburg) , PS048, PS056, PS057

Schilli, P. (Freiburg) , YIA05

Schiopu, S. (Munich) , PS082 Schleicher, S. (Köln) , PS032, PS034, PS035

Schlosser, S. (Regensburg) , PS016, PS017, PS059

Schmader, K. (Durham) , PS103

Schmid, S. (Regensburg) , PS080

Schmidt, L. (Mainz) , PS117

Schmitt, A. (Heidelberg) , PS046

Schmitt, M. (Heidelberg), PS046

Schneider, C. (Homburg) , YIA03

Schneider, M. (Düsseldorf) , PS009

Scholtis, A. (Straubing) , YIA06

Scholz, S. (Homburg) , PS028, PSO43, PS060

Schreiyäck, C. (Gießen) , PS065

Schrenk, D. (Kaiserslautern) , PS038

Schreyögg, J. (Hamburg), PS008

Schulte, B. (Hannover) , PS040

Schultis, W. (Weiden/Opf.) , PS062

Schulz, K. (Brake) , PS116

Schulz, H. (Chemnitz) , PS095

Schulze, J. (Frankfurt am Main),

PS109

Schulze-Bahr, E. (Münster) , YIA07

Schumacher, B. (Kaiserslautern),

PS001

Schumann, F. (Berlin) , PS030

Schürholz, T. (Rostock) , YIA02

Schütte, K. (Magdeburg) , PS031
Schwartz, C. (Düsseldorf), PS009

Schwarz, V. (Homburg) , PS099

Schwenk, B. (Esslingen) , PS081

Sehlen, S. (Berlin) , PS004

Sembach, L. (Kaiserslautern) , PS001

Serr, J. (Berlin) , PS067

Seufert, J. (Freiburg) , PS063

Sha-Hosseini, K. (Köln) , PS032,

PS034, PS035

Shi, C. (München) , PS026

Shirneshan, K. (Göttingen) , PS107

Shrubsole, M. (Nashville) , YIA01

Sibelius, U. (Gießen) , PS083

Siebald, B. (Frankfurt am Main),

PS109

Siebenlist, G. (Erlangen) , PS024

Singh, J. (Köln) , PS032, PS034, PS035

Sippel, C. (Bonn) , PSO02

Smetana, J. (Hradec Kralove) , PS100

Söhler, S. (Marburg) , PS086, YIA04

Solovyeva, E. (Frankfurt (Main))

PS048, PS056

Sommerer, C. (Heidelberg) , PS046

Sossalla, S. (Regensburg) , PS047

Speer, C. (Heidelberg) , PS046, PS074

Spielmanns, M. (Wald) , PS090,

PS091, PS092

SRIKANTH, V. (Kochi, Kerala) , PS021,

PS022, PS101, PS102, PS118

Staerkle, A. (Zürich) , PS113

Stampfer, M. (Tübingen) , PS068

Stehle, T. (Regensburg) , PS047

Steinborn, A. (Heidelberg) , PS046

Steinhagen-Thiessen, E. (Berlin),

PS030, PS042

Stelzer, D. (Freiburg) , PS104

Storz, G. (Freiburg) , YIA05

Straub, B. (Mainz) , PS012

Streckfuss-Bömeke, K. (Göttingen)

,PS047

Stromberger, C. (Berlin) , PS005

Stuschke, M. (Essen) , PS005

Südhoff, T. (Passau) , PS011

Sydor, S. (Magdeburg) , PS015

\section{T}

Tacke, F. (Aachen), YIA02

Taube, C. (Essen) , PS089

Tews, H. (Neustadt) , PS078

Theobald, M. (Mainz) , PS115, PS117

Thieß, K. (Berchtesgaden) , PS097

Thomsen, H. (Heidelberg), YIA01

Thorgeirsson, S. (Bethesda/ Was-

hington DC) , PS012, PS020

Tinhofer, I. (Berlin) , PS005

Tirilomis, P. (Göttingen) , PS047

Tobschall, M. (Erlangen), PS098

Todica, A. (Munich) , PS082

Tomczak, M. (Düsseldorf) , PS009

Townsend, R. (Philadelphia) , PS043

Trinh, B. (Basel) , PS113

Trudzinski, F. (Homburg) , PS086

Trunk, J. (Lüdenscheid) , PS108

Tsiklauri, L. (Bad Nauheim) , PS077
Tüffers, J. (Marburg) , PS087

U

Uhle, F. (Heidelberg) , PS074

Ukena, C. (Homburg) , PS043, PS060,

PS069

Ulmer, H. (Mainz) , PS096

Uusinarkaus, K. (Colorado Springs), PS103

\section{V}

Van den Steen, P. (Wavre) , PS100 van Ewijk, R. (Mainz) , PS104 Vandieken, E. (Homburg), YIA03 Velasco, C. (Sankt Augustin) , PS009 Velthuis, L. (Mainz) , PS104 Verjee, S. (Kaiserslautern) , PS038 Vilchez-Vargas, R. (Magdeburg) PS015

Vitali, F. (Erlangen) , PS061 Vogelmeier, C. (Marburg) , PS044, PS087, PS088, YIA04

Vogg, J. (Regensburg) , PS080 Vogl, T. (Frankfurt am Main) , PS109 von Arnim, U. (Magdeburg) , PS013, PS019

von der Grün, J. (Frankfurt) , PS005 von Rauchhaupt, E. (Munchen), PS114

von Siemens, M. (München) , PS086 Vukadinovic, D. (Homburg) , PS028, PS043

W

Wagenpfeil, S. (Homburg) , PS043 Wagner-Drouet, E. (Mainz) , PS115, PS117

Waldmüller, S. (Tübingen) , PS068 Wallacher, H. (Nierstein) , PS084 Wang, L. (Heidelberg) , PS046 Wangler, J. (Mainz) , PS104 Waschki, B. (Grosshansdorf und Hamburg) , PS044, PS088 Watanabe, D. (Nagakute) , PS100 Watz, H. (Grosshansdorf) , PS044, PS086, PS088, YIA04

Weber, Y. (Tübingen) , PS002 Weckx, L. (Sao Paulo) , PS100 Wegener, T. (Weinheim) , PS032, PS034, PS035

Weigand, M. (Heidelberg) , PS074 Weigl, K. (Heidelberg) , YIA01 Welte, T. (Hannover) , PS086, PS088, YIA04

Wenisch, S. (Giessen) , PS077

Wenning, C. (Essen), PS015 Wenzelburger, F. (Kaiserslautern), PS001 


\section{Autorenregister}

Werner, C. (Homburg) , PS099

Werner, J. (Giessen) , PS077

Wessendorf, T. (Essen) , PS089

Wiedemann, S. (Basel) , PS113

Wolf, M. (Kassel) , PS029

Wölfinger, P. (Mainz) , PS115

Wörns, M. (Mainz) , PS020, PS104

Wosch, F. (Hanau) , PS063

Wübbolding, M. (Hannover), PS040

Wujak, L. (35392) , PS085

Würf, V. (Munich) , PS114

Wygrecka, M. (Gießen) , PS085

Wyrwich, W. (Berlin) , PS004

Y

Yard, B. (Mannheim) , PS106

Yazdani, B. (Mannheim) , PS045

Yu, X. (Aachen) , PS023

Z

Zacherl, M. (Munich) , PS082

Zadoyan, G. (Köln) , PS032, PS034,

PS035

Zahaf, T. (Wavre) , PS100

Zawada, A. (Homburg) , PS057

Zeier, M. (Heidelberg) , PS046, PS074

Zeller, T. (Hamburg) , PS088

Zenner, C. (Frankfurt) , PS048

Zeymer, U. (Ludwigshafen) , PS027,

PS055

Ziegler, M. (Berlin) , PS004

Zielen, S. (Frankfurt am Main) ,

PS109

Zimmer, C. (Saarbrücken) , PS041

Zimper, G. (Kirkel) , PS104

Zippel, C. (Heidelberg) , PS067

Zippel-Schultz, B. (Berlin) , PS001,

PS006

Zipprich, A. (Halle) , YIA03

Zips, D. (Tübingen) , PS005

Zopf, Y. (Erlangen) , PS072, PS098

Zotz, R. (Bitburg) , PS001

Zucker, M. (Gießen) , PS085

Zwack, L. (München) , PS002

Zwerenz, B. (Koblenz) , PS104 